\title{
STRANGENESS IN RELATIVISTIC HEAVY ION COLLISIONS
}

\author{
P. KOCH, B. MÜLLER and J. RAFELSKI
}

Institut für Theoretische Physik der Universität Frankfurt, Postfach 111932, 6000 Frankfurt 11, W. Germany

Institute of Theoretical Physics and Astrophysics, University of Cape Town, Rondebosch 7700, Cape, R.S.A.

and

Gesellschaft für Schwerionenforschung (GSI), Postfach 110541, 6100 Darmstadt 11, W. Germany

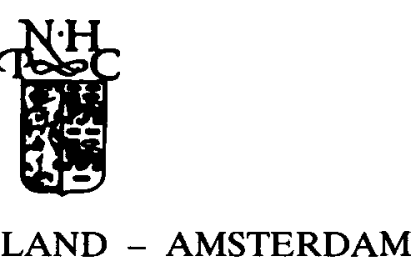




\title{
STRANGENESS IN RELATIVISTIC HEAVY ION COLLISIONS
}

\author{
P. KOCH, B. MÜLLER and J. RAFELSKI \\ Institut für Theoretische Physik der Universität Frankfurt, Postfach 111932, 6000 Frankfurt 11, W. Germany \\ Institute of Theoretical Physics and Astrophysics, University of Cape Town, Rondebosch 7700, Cape, R.S.A. \\ and \\ Gesellschaft für Schwerionenforschung (GSI), Postfach 110541, 6100 Darmstadt 11, W. Germany
}

Received March 1986

Contents:

1. Introduction 169

1.1. Overview 169

1.2. Bagged QCD 172

1.3. Ideal gas of quarks and gluons 173

1.4. Phase boundary of quark-gluon plasma $\quad 176$

1.5. Evolution of quark-gluon plasma 178

2. Strange quark mass and hadronic spectrum 181

2.1. Phenomenological mass formula 181

2.2. Fitting procedures $\quad 182$

2.3. Strange quark energy and mass 183

3. Heavy quark production in quark-gluon plasma 186

3.1. Quark pair production 186

3.2. Results for static quark-gluon plasma 192

3.3. Influence of volume expansion 195

3.4. Influence of Pauli blocking of final states 198

3.5. Pre-equilibrium production of $J / \psi$ particles 201

4. Conversion from the quark-gluon phase to the hadronic phase (Hadronization of quark-gluon plasma)

4.1. Hadronization scenarios

4.2. Fragmentation of quarks and gluons

4.3. Combinatoric break-up model 208

4.4. Hadrons from quark-gluon plasma 210

5. Strange hadrons in hadronic gas - Evolution and formation 217
5.1. Relevant reactions 217

5.2. Rate equations for the production of strange particles 219

5.3. Approach to equilibrium of strangeness abundance in hot hadronic matter

6. Strangeness abundance in hadronic gas in chemical equilibrium

6.1. Grand canonical treatment

6.2. State of chemical equilibrium in hadron-nucleus reactions

6.3. Relative chemical equilibrium

6.4. Measurement of the baryochemical potential

7. Strangeness evolution in an expanding fireball 240

7.1. Fireball scenario 240

7.2. Strange particle abundances: Baryon-rich plasma 245

7.3. Numerical results 247

8. Highlights 250

Appendix A: Master equations for strangeness evolution in hadronic gas 252

Appendix B: Parameterization of strangeness production cross sections in hadronic gas 255

References $\quad 259$

Notes added in proof 261

Abstract:

Abundances of strange antibaryons formed in nuclear collisions at above $10 \mathrm{GeV} / \mathrm{A}$ are considered as a most accessible diagnostic tool for the study of the possible formation and physical properties of the quark-gluon plasma phase of hadronic matter. In this report we describe the current status and develop a dynamical approach in order to describe strange particle formation in nuclear collisions at high energy.

Single orders for this issue

PHYSICS REPORTS (Review Section of Physics Letters) 142, No. 4 (1986) 167-262.

Copies of this issue may be obtained at the price given below. All orders should be sent directly to the Publisher. Orders must be accompanied by check.

Single issue price Df. 64.00 , postage included. 


\section{Introduction}

\subsection{Overview}

Nearly all matter around us is built of up (u) and down (d) quarks. However, as soon as adequate excitation energy becomes available in hadronic interactions, it becomes apparent that further quark flavours exist and are easily accessible. The lightest of the heavy flavours of matter was called 'strange' as it appeared so to its discoverers, and today this name is associated with the strange $\bar{s}$-antiquark. s-quarks are identical in their properties (charge $q=-\frac{1}{3}$, baryon number $b=+\frac{1}{3}$, spin $\frac{1}{2}$, colour $\underline{3}$ ) to the d-quarks but different in mass.

In the laboratory experiments excitation of strange matter is understood in terms of matterantimatter formation as it is the $s-\bar{s}$ quark pair which is created in reaction processes by strong or electromagnetic interactions. The formation mechanism of strange quarks is highly correlated to the reaction mechanism governing the hadronic collisions - most of this review will deal with our current understanding of the strangeness generation in nuclear collisions and how occurrence of various strange and multiply strange hadrons can tell us the story about the formation of the new phase of matter, the quark-gluon plasma [RH80, Ra81, Ra82, RM82, Ra83, Ra84]. Alternate approaches to diagnose quark gluon plasma, e.g. by dilepton spectra [DG81, Ch82, SC85, HK85] are also of considerable interest, but it has not been established beyond reasonable doubt that in this case the usual backgrounds will permit measurement.

We will not discuss further other interactions and properties of strange quarks such as the Cabibbo mixing of the down and strange quarks with respect to the weak interactions. Note, however, that this would become relevant if the difference in mass of $d$ and $s$ quarks would be generated, e.g. by the properties of the vacuum state of strong interactions. Should this speculation be true, it is quite imaginable that in nuclear collisions various fundamental properties of strange quarks could be altered. This remark is a particular case of the observation that the physical properties of matter and even the appearance of laws of physics may undergo a drastic change within the environment of a quark-gluon plasma. This we take as primary motivation for a detailed and exhaustive study of the properties of the ground state of strong interactions and its melted form, the quark-gluon plasma. We will describe how certain strange particle abundances created in nuclear collisions can be used as a tool to study the properties of this, still hypothetical form of matter.

Our view of the relativistic nuclear collision reaction [DR82] is in agreement with the recently observed large nuclear stopping power [BG84] which implies that a domain in space arises in a centre of momentum frame of two nuclei in which an important part of the longitudinal energy is transferred to transverse degrees of freedom, as also recently confirmed experimentally [KS85]. Such regions of space we call 'fireball'. Our reaction picture and the mentioned experimental results contradict earlier theoretical proposals [AKM80] based on $\mathrm{p}-\mathrm{p}$ collision phenomenology and assuming essential nuclear transparency. More recent theoretical studies [CK84, DGS85] find high p-A stopping power and therefore suggest that nuclear collisions at energies under consideration will be truly inelastic.

The physical variables characterising the fireball domain are: energy (density), baryon number (density), entropy (density), and volume rather than the usual individual degrees of freedom of particles. The methods required in the theoretical study of these quantities involve straightforward generalisations of the usual statistical thermodynamic theory. However, the fireballs are rather isolated and not in temperature or particle 'baths'. Further, the size of the fireball, and in particular, its baryon number may be relatively small. Hence, one may justly question the validity of the statistical approach. 
The concept of 'temperature' is almost certainly useful in this context, due to the large number of particles contained in a hadronic fireball. It is less obvious whether particle conservation laws may be considered in the framework of the grand canonical ensemble through the introduction of various associated chemical potentials $\mu_{i}$. Exact 'counting' of particle members in the 'canonical' ensemble in comparison with the statistical average in the 'grand canonical' approach leads to correction factors of the order $1 / N$, where $N$ is the relevant number of particles [HR84]. Here, $N$ may be the baryon number $b$, strangeness $S=s-\bar{s}=0$ or even be a synonym for required colour conservation. Correction factors for exact strangeness and colour conservation have been studied [RD82, EGR83, EGR84, DGER85] and it has been found that for fireball volumes corresponding to a size of $2 \mathrm{fm}$ in radius or more, these corrections are not significant. We will be content hence with the grand canonical approach and use the baryochemical potential $\mu_{\mathrm{b}}$ next to temperature $T$, as the physically observable statistical variables throughout this work.

We further note that all particle quantum numbers that are invariant under the strong interaction are conserved on the timescale of interest to us $\left(10^{-24} \mathrm{~s} \leq t \leq 10^{-21} \mathrm{~s}\right)$. Such quantum numbers are, aside from baryon number $b$, electric charge $Q$, and all flavour quantum numbers, in particular, strangeness $S$. The third component of isospin is hence also conserved due to the Gell-Mann-Nishijima relation, but the conservation of isospin may be violated to some extent due to electromagnetic interactions (we note that the isospin-violating reactions, e.g. the decay of $\Sigma^{0} \rightarrow \Lambda \gamma$ have a characteristic time constant of e.g. $\left.10^{-20} \mathrm{~s}\right)$.

A prerequisite in our work is rather precise knowledge of certain empirical properties of strange quarks in a large quark bag. We will discuss at length in the next section how the study of the spectrum of strange particles helps in understanding the mass of confined strange quarks [AR84, AR85]. While the physical origin of the strange quark mass $m_{\mathrm{s}}$ remains a puzzle, its precise value is of considerable importance to us because $2 m_{\mathrm{s}}$ is the threshold for the formation of a strange quark pair. This threshold is near to the hadronic 'temperature' $T \sim 160 \mathrm{MeV}$ observed in the slopes of the particle spectra [Ha65, HR68] and which is a measure of the mean energy available per particle in hadronic reactions. Consequently, the detailed balance of the energy available in a reaction to form the strange quark pair and the reaction mechanism must be carefully considered in order to quantify properly the resulting strange particle abundances.

The first and foremost issue at hand concerning the nucleus-nucleus collision relates to the internal structure of the formed fireball:

1. Does it consist of individual hadrons with short range correlations between the constituent quarks? Is the nuclear collision reaction therefore governed by interactions between individual hadronic resonances?

2. Or, does it consist of the quark-gluon plasma phase where short range colour correlations are broken and in which quarks and in particular, gluons are the active constituents?

Although in our work the latter option will always be tacitly assumed, it is one of the main points we will make here that the distinction of the quark-gluon plasma from the hadronic gas is facilitated by the excitation of numerous gluons, a situation which can only occur in the plasma phase. But since gluons carry the (confined) charge of strong interactions, colour, their detection in an experiment can only be indirect. We show in section 3 that gluons are very efficient in generating strange quark-antiquark pairs in quark-gluon plasma and hence transitory presence of gluons may be inferred from the appearance of anomalously high strange particle abundance which will practically saturate the available strange quark phase space.

In order to properly appreciate these remarks, one has first to recognize that the basic subprucess for 
strange quark production, namely the pair production process $\mathrm{gg} \rightarrow \mathrm{s} \overline{\mathbf{s}}$, is, in principle, the same for both phases of hadronic matter described above. In the case of well-separated individual hadrons with the nonperturbative ('true') QCD vacuum in between, the above-mentioned reaction can only take place during the actual collision process of two individual hadrons, which means that strange quark production experiences severe constraints in space and time. Furthermore, all initial and final state hadrons are colour singlets and the effective number of the available degrees of freedom is greatly reduced in comparison to the quark-gluon phase.

In the plasma phase there is not only more rapid strangeness production - we will show that the substantially higher strange quark abundance per unit of volume leads to abundant formation of multiply strange antibaryons when the plasma state fragments and recombines to form individual hadrons. In the baryon-rich regime of quark-gluon plasma the $\overline{\mathrm{s}}$-quarks are more abundant than $\overline{\mathrm{u}}$ or $\overline{\mathrm{d}}$ quarks with the consequence that formation of antibaryons with high strangeness content is facilitated during the conversion to the hadronic gas of the plasma phase (see section 4). We develop in this volume the quark-gluon fragmentation-recombination model for plasma dissociation and the numerous fragmentation processes provide additional antiquarks required in the formation of strange antibaryons.

One of the important results discussed here (section 5) is the fact that during the expected nuclear collision reaction time of ca $10^{-22} \mathrm{~s}$ the strangeness produced in individual hadronic collisions in the thermalised phase will not fill the available phase space - however, we also show that strange particles are rather efficiently distributed among various individual hadronic states, e.g. s-quarks will be found either in antikaons $\bar{K}(\mathrm{sq})$ or hyperons $\mathrm{Y}(\mathrm{sqq})$ in dependence on the value of the chemical potential $\mu_{\mathrm{b}}$ (baryon density), but the absolute strangeness abundance may be substantially less than the phase space limit unless quark-gluon plasma state is formed. This phenomenon, which is well-known from nucleon-nucleon collisions has recently been shown to persist in reactions among light nuclei, such as $\mathrm{d}$ and $\alpha$ [Ak85]. In these reactions the usual $\mathrm{K} / \pi$ ratio of about 11 percent is observed, even at large transverse momenta indicating that no change in reaction mechanism has occurred. Relative chemical equilibrium is more easily established in the hadronic gas phase than the absolute chemical equilibrium saturation of phase of strange particles, which depends strongly on the governing reaction mechanisms. One can use the relative chemical equilibrium relations between kaons and hyperons to measure the baryochemical potential-we will discuss these and related issues in section 6 . However, even the relative chemical equilibrium will not always be established especially in case that the dense hadronic state contains numerous, normally rare, antibaryons which signal the occurrence of quark-gluon plasma.

The final aspect of our work relates to the transition from the dense state of matter to a relatively thin gas of particles which will ultimately, without further interactions, reach the observer. In section 7 we calculate the abundances of strange particles arising upon the formation of quark-gluon plasma under two extreme assumptions on the dynamics on the expansion-hadronization process: (a) all entropy of the plasma is spent in expansion; (b) it is converted almost exclusively into new particles through fragmentation processes of quarks and gluons. We favour the latter case as being more representative of the hadronic dynamics, since a large particle abundance then results - in case (a) the entropy is used to increase the mean energy per particle in the final state through the process of collective expansion. Numerous fragmentations of gluons in the case (b) provide a ready supply of $\bar{q}$ to complete the formation of antihyperons further enhancing their extraordinarily high abundance.

We turn to describe several underlying assumptions about strong interactions and nuclear collisions, which we will use when required in this work. 


\subsection{Bagged $Q C D$}

We will tacitly assume bagged QCD as the underlying picture of the interactions in study. This presupposes that the 'true' vacuum state is structured such that any coloured particle cannot freely propagate beyond a distance of about $1 \mathrm{fm}$. This state can be converted into the 'perturbative vacuum' at the expense of an energy density $B$. This notion has been introduced originally in the MIT bag model, but initially in a different context [Ch74, Jo75]. The value of $B$ is estimated phenomenologically from a fit to the hadronic spectrum [deG75, AR84, AR85] or from sum rule considerations [Sh81] (for a recent review see [RRY85]) which give the range

$$
B=[(140-210) \mathrm{MeV}]^{4}=(50-250) \mathrm{MeV} / \mathrm{fm}^{3} .
$$

The central assumption of the quark-bag approach is that inside a hadron where quarks are found, the true vacuum structure is displaced or destroyed and coloured particles propagate easily. One can turn this point around: quarks can only propagate in domains of space in which the true vacuum structure is absent. This statement constitutes, if taken to the extreme, a resolution of the quark confinement problem. The remaining difficult and unsolved problem is to understand the degree of incompatibility of 'free' quarks with the true vacuum structure. Examples of similar behaviour in ordinary physics are easily found: e.g., a light wave is reflected from a mirror surface (not perfectly!); magnetic field lines are expelled from superconductors; etc. It has been argued [Le81] that the true vacuum state of quantum chromodynamics (QCD) is a perfect dielectric.

It is interesting to note that the bag constant $B$ has a further influence: a negative pressure $P=-B$ as seen from the perturbative vacuum, acts on the surface between the true and the perturbative vacuum. Hence, in the absence of other forces an empty, excited space domain containing the perturbative vacuum would quickly vanish. In this picture of hadronic structure and quark confinement, all colourless assemblies of quarks, antiquarks and gluons can form quasi-stationary states, a 'quark bag'. In perturbative vacuum the laws of perturbative quantum chromodynamics apply and we find there relatively weakly interacting perturbative quanta of QCD.

As this type of understanding of strongly interacting particles is quite commonly accepted today, we will not repeat all well-known arguments that lead to this approach. Nor will we consider the refinements not of essential importance to the main line of our work. These are mainly brought about by the difficulties encountered in the incorporation of pions in this schematic description. However, we note that nuclear collisions, in which internally excited large quark-gluon bags ('quark-gluon plasma') are formed, test the above conceptual understanding from a quite different angle as for the first time large regions of space devoid of structured vacuum will be created. This process of vacuum melting is, in our view, the primary objective of the high energetic nucleus-nucleus collision experiments. However, it is fitting to note at this point that neither absence of a phase transition in the thermodynamic sense between the hadronic vacua, or even the possible existence of deconfined quarks around us will greatly influence our considerations. All we presuppose is that the melted state contains quark-gluon like excitations with established QCD properties.

We will invoke the above qualitative understanding of the hadronic structure when in the next section (section 2) we turn to study, in the context of relativistic bag models, the strange quark mass $m_{\mathrm{s}}$. This is done by exploring in a systematic fashion the compatability of the bagged QCD picture and the spectrum of strongly interacting particles. We determine that the value of $m_{\mathrm{s}}$ we should employ is in the order of $160-180 \mathrm{MeV}$. 
Another relevant parameter in our work is the strong coupling constant $\alpha_{\mathrm{s}}$. It controls, among others, the coupling of the strange quarks to the light flavours $\mathrm{u}, \mathrm{d}$ and the gluons. The value of $\alpha_{\mathrm{s}}$ is changing with momentum transfer and it is different in timelike and spacelike regions. In production of strange quark pairs, we are exploring the timelike domain of momenta in which the strong coupling has been found to be weaker than in spacelike regions. We will therefore consider a value in the range $\alpha_{\mathrm{s}}=0.4-0.8$ as being the appropriate one, in view of the relatively small momenta involved (less than $1 \mathrm{GeV} / c$ on the average). Recall that $\alpha_{\mathrm{s}} \sim 0.14-0.20$ is found in $\mathrm{e}^{+} \mathrm{e}^{-} \rightarrow$ hadrons experiments at $\sqrt{s}>10 \mathrm{GeV}$ [DR85]. The smallness of $\alpha_{\mathrm{s}}$ justifies the use of ideal gas expression to gain a first insight into the properties of the quark-gluon plasma, as is also supported by results of numerical calculations in the context of lattice SU(3) gauge theory. Further, as pointed out by Carruthers [Ca83] the collective phonon oscillations in plasma just about compensate for the suppression of low energy perturbative modes of gluons, providing an explanation for this 'precocious' validity of the perturbative ideal gas expressions.

\subsection{Ideal gas of quarks and gluons}

As the quark-gluon plasma is a state composed of highly relativistic particles, where particleantiparticle pairs can be created and destroyed at liberty, particle numbers as such, are not conserved, only the difference in the number of particles and antiparticles. However, as four-momentum conservation still holds, a co-moving volume element at equilibrium can be described by a four-velocity $u^{\mu}$ and a local temperature $T$, so that particle densities in phase space depend only on the conventional Boltzmann factor: $\exp \left(\beta_{\mu} p^{\mu}\right)$, where the four-vector $\beta_{\mu}$ is given by [To68]

$$
\beta_{\mu}=\frac{1}{T} u_{\mu}
$$

In the rest frame of the co-moving volume element one has, as usual, $\beta_{\mu}=(1 / T, \mathbf{0})$.

Let us now discuss, in the rest frame of the plasma, the various constituent particle species of the quark-gluon plasma. For gluons there is no conservation law restricting their number, so their density, in ideal gas approximation, is given by the phase-space integral over the Bose distribution function for massless particles $(\zeta(3)=1.20206 \ldots)$

$$
\rho_{\mathrm{g}}=g_{\mathrm{g}} \int \frac{\mathrm{d}^{3} p}{(2 \pi)^{3}}\left(\mathrm{e}^{\beta|p|}-1\right)^{-1}=g_{\mathrm{g}} \frac{T^{3}}{\pi^{2}} \zeta(3) .
$$

Here $g_{\mathrm{g}}=8 \times 2$ (colour $\times$ spin) is the degeneracy factor of gluon states. We have

$$
\rho_{\mathrm{g}}=16\left(T^{3} / \pi^{2}\right) \zeta(3)
$$

and we note that $\alpha_{\text {s }}$ corrections to eq. (1.3) and further relations of similar character are known explicitly, see e.g. [Ch78, EGR80, RD83, RD85] expressions below. The energy density associated with gluons is $\left(\zeta(4)=\pi^{4} / 90=1.08232 \ldots\right)$

$$
\varepsilon_{\mathrm{g}}=g_{\mathrm{g}} \int \frac{\mathrm{d}^{3} p}{(2 \pi)^{3}}|p|\left(\mathrm{e}^{\beta|p|}-1\right)^{-1}=g_{\mathrm{g}} \frac{3}{\pi^{2}} T^{4} \zeta(4)=\frac{8}{15} \pi^{2} T^{4}
$$


For quarks, we have net conservation of each flavour separately, i.e. of the number of quarks minus the number of antiquarks. This is accounted for by introducing a chemical potential $\mu_{f}$, where $f=$ $\mathrm{u}, \mathrm{d}, \mathrm{s}, \ldots$ denotes each individual flavour. If the quark mass is $m_{f}$, the net number of quarks of flavour $f$ is, according to the Fermi statistical weight

$$
\nu_{f} \equiv \rho_{f}-\rho_{\bar{f}}=g_{f} \int \frac{\mathrm{d}^{3} p}{(2 \pi)^{3}}\left[\left(\exp \left\{\beta\left(\sqrt{p^{2}+m_{f}^{2}}-\mu_{f}\right)\right\}+1\right)^{-1}-\left(\exp \left\{\beta\left(\sqrt{p^{2}+m_{f}^{2}}+\mu_{f}\right)\right\}+1\right)^{-1}\right]
$$

where we have made use of the fact that the chemical potentials of quarks and antiquarks are of equal magnitude and opposite sign.

Equation (1.5), in general, cannot be evaluated in closed form. For the light quarks, $f=\mathrm{u}$, $\mathrm{d}$, we may, however, put $m_{f} \simeq 0$, and then we find, with $g_{f}=2_{\mathrm{s}} \cdot 3_{\mathrm{c}}=6$ for spin and colour:

$$
\nu_{f}=\mu_{f} T^{2}+\left(1 / \pi^{2}\right) \mu_{f}^{3} \quad(f=\mathrm{u}, \mathrm{d})
$$

(for details see [RD85, MU85]). When the quark-gluon plasma state arises from symmetric nuclear matter, i.e. $Z=\frac{1}{2} A$, the number of up- and down-quarks must be equal, so that

$$
\mu_{\mathrm{u}}=\mu_{\mathrm{d}} \equiv \mu_{\mathrm{q}}=\frac{1}{3} \mu_{\mathrm{b}}
$$

and the baryon density $\rho_{\mathrm{b}}$ is

$$
\rho_{\mathrm{b}}=\frac{1}{3}\left(\nu_{\mathrm{u}}+\nu_{\mathrm{d}}\right)=\frac{2}{3} \nu_{f}\left(\mu_{\mathrm{b}} / 3\right) \text {, }
$$

where $\mu_{\mathrm{b}}$ is the baryon chemical potential.

Strange quarks are created in pairs only, and hence we have

$$
\nu_{\mathrm{s}}=n_{\mathrm{s}}-n_{\overline{\mathrm{s}}}=0,
$$

which implies that $\mu_{\mathrm{s}}=0$, c.f. eq. (1.5). Expanding the Fermi function in eq. (1.5), we then have in view of $m_{\mathrm{s}} / T \geq 1$ :

$$
n_{\mathrm{s}}=n_{\overline{\mathrm{s}}}=g_{\mathrm{s}} \int \frac{\mathrm{d}^{3} p}{(Z \pi)^{3}} \sum_{n=1}^{\infty}(-)^{n-1} \exp \left(-n \beta \sqrt{p^{2}+m_{\mathrm{s}}^{2}}\right)=\frac{3}{\pi^{2}} T^{3}\left(\frac{m_{\mathrm{s}}}{T}\right)^{2} \sum_{n=1}^{\infty} \frac{(-)^{n-1}}{n} K_{2}\left(n \frac{m_{\mathrm{s}}}{T}\right),
$$

and again $g_{\mathrm{s}}=2 \mathrm{~s} \cdot 3_{\mathrm{c}}=6$. In the so-called Boltzmann approximation we only retain the $n=1$ term:

$$
n_{\mathrm{s}}=n_{\overline{\mathrm{s}}} \approx \frac{3}{\pi^{2}} T m_{\mathrm{s}}^{2} K_{2}\left(\frac{m_{\mathrm{s}}}{T}\right) \text {. }
$$

We will now compare this to the light antiquark density ( $\overline{\mathrm{q}}$ stands for either $\overline{\mathbf{u}}$ or $\overline{\mathrm{d}}$ ) 


$$
n_{\overline{\mathrm{q}}}=6 \int \frac{\mathrm{d}^{3} p}{(2 \pi)^{3}} \frac{1}{\left(\exp \left(|p| / T+\mu_{\mathrm{q}} / T\right)+1\right)} .
$$

The integral (1.11) can be expanded in a manner analogous to eq. (1.9), and one obtains the following ratio of strange to nonstrange antiquarks:

$$
n_{\overline{\mathrm{s}}} /\left(n_{\overline{\mathrm{u}}}+n_{\overline{\mathrm{d}}}\right) \approx \frac{1}{2}\left(m_{\mathrm{s}} / T\right)^{2} K_{2}\left(m_{\mathrm{s}} / T\right) \exp \left(\mu_{\mathrm{b}} / 3 T\right)
$$

which is depicted in fig. 1.1. There is a strong suppression of light antiquarks in the limit of large $\mu_{\mathrm{b}}$, that is in the baryon-rich quark gas.

The energy density carried by light quarks is found explicitly, assuming again negligible rest mass:

$$
\begin{aligned}
\varepsilon_{\mathrm{q}} & =\varepsilon_{\mathrm{u}}+\varepsilon_{\overline{\mathrm{u}}}+\varepsilon_{\mathrm{d}}+\varepsilon_{\overline{\mathrm{d}}}=12 \int \frac{\mathrm{d}^{3} p}{(2 \pi)^{3}}|p|\left[\left(\exp \left(\beta|p|-\beta \mu_{\mathrm{q}}\right)+1\right)^{-1}+\left(\exp \left(\beta|p|+\beta \mu_{\mathrm{q}}\right)+1\right)^{-1}\right] \\
& =\frac{7 \pi^{2}}{10} T^{4}+3 \mu_{\mathrm{q}}^{2} T^{2}+\frac{3}{2 \pi^{2}} \mu_{\mathrm{q}}^{4} .
\end{aligned}
$$

For an ultrarelativistic gas of particles, even in the presence of perturbative interactions, the pressure of gluons and light quarks can generally be calculated according to the law

$$
P=\frac{1}{3} \varepsilon
$$

while the entropy density is obtained by evaluating

$$
s=\partial P /\left.\partial T\right|_{\mu}
$$

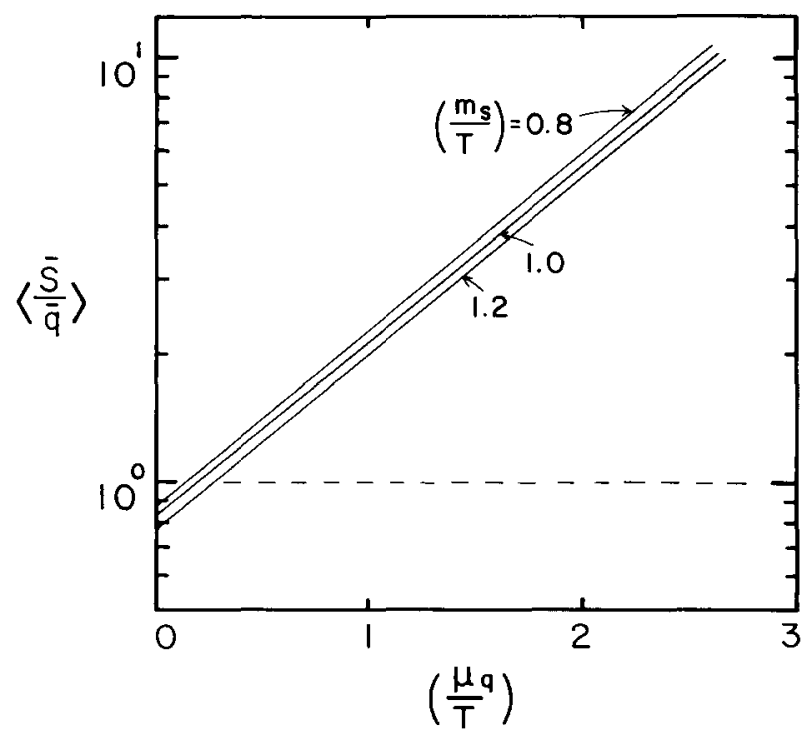

Fig. 1.1. Abundance of strange (or antistrange) quarks relative to the light quark abundance as function of $\mu_{\mathrm{q}} / T$ for several choices of $m_{\mathrm{s}} / T=0.8$, $1,1.2$. 
We shall need just below the relation (1.14) in order to estimate the phase transition back to hadronic gas, while entropy plays an important role in estimating the particle number abundances [GR85]. A rather complete explicit list of all relations $(1.14,1.15$, etc. $)$ including $\mathrm{O}\left(\alpha_{\mathrm{s}}\right)$ corrections is easily found in literature to which the reader is referred to further study (see e.g. [RD85, MU85, CGS86]). Historically, the first discussion of quark matter known to us is that of Carruthers [Ca73].

\subsection{Phase boundary of quark-gluon plasma}

It is convenient, though rather unrealistic, to visualize the hot excited nuclear matter formed in relativistic nuclear collisions as if in contact with a thermodynamical bath of temperature $T$ and baryochemical potential $\mu_{\mathrm{b}}$.

We recall that, given this assumption, the extensive variables, viz., energy, volume, baryon number of the fireball, etc., can be expressed by intensive quantities: The temperature $T$ is a measure of energy per degree of freedom; the baryon chemical potential $\mu_{\mathrm{b}}$ controls the mean baryon density. The statistical quantities such as entropy (a measure for the number of available states), pressure, heat capacity, etc., also will be functions of $T$ and $\mu_{\mathrm{b}}$, and describe the state of nuclear matter. Any theoretical description of the two quite different phases, viz., the hadronic gas and the quark-gluon plasma, must allow for the formation of numerous hadronic resonances on the one side, which then at sufficiently high energy density, dissolve into the state consisting of their deconfined constituents: quarks and gluons.

It is important for the later modelling of the quark-gluon plasma state dissociation into the hadronic gas to keep in mind, as an illustration of what is happening, the $p-V$ diagram shown in fig. 1.2. Here we distinguish three domains. The hadronic gas region is approximately a Van der Waals gas where the pressure rises with reduction of the volume. When the internal excitation rises, the individual hadrons begin to cluster. This reduces the increase in the pressure, since a smaller number of particles exercises a smaller pressure. In a complete description of the different phases we have to allow for a coexistence of hadrons with the plasma state in the sense that the internal degrees of freedom of each cluster, i.e. quarks and gluons, contribute to the total pressure even before the dissolution of individual hadrons. This, indeed, becomes necessary when the clustering overtakes the compressive effects and the hadronic gas pressure falls to zero as $V$ reaches the proper volume of hadronic matter. At this point the pressure rises again very quickly, since in the absence of individual hadrons we now compress only the hadronic constituents. It is interesting to follow the path taken by an isolated quark-guon plasma fireball in the baryon density-temperature $\rho_{\mathrm{b}}-T$ plane. Several cases are depicted in fig. 1.3 . In the Big

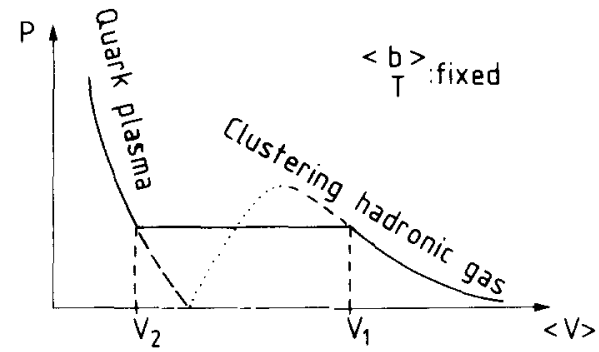

Fig. 1.2. $p-V$ diagram for the gas-plasma first order transition, with the dots indicating a model-dependent, unstable domain between overheated and undercooled phases.

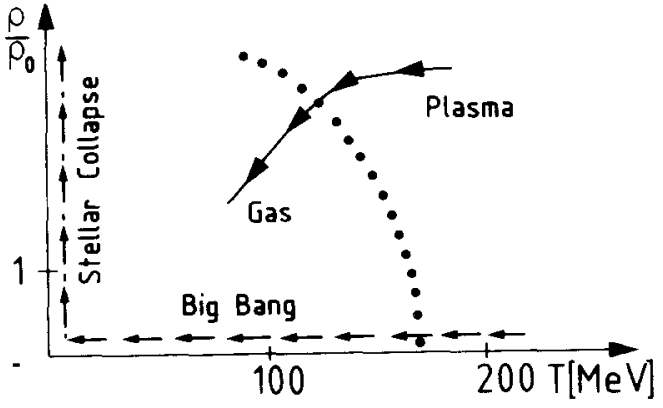

Fig. 1.3. Paths taken in the $\rho_{\mathrm{b}}-T$ plane by different physical events. 
Bang expansion of the early universe, the cooling shown by the dashed line occurs in a universe in which most of the energy is in the form of radiation. Hence, the baryon density $\rho_{\mathrm{b}}$ is quite small compared to the entropy density. In stellar collapse leading to cold neutron stars, we follow the dash-dotted line parallel to the $\rho_{\mathrm{b}}$-axis. The compression is accompanied by little heating.

In contrast, in nuclear collisions, probably the central area covering most of the $\rho_{\mathrm{b}}-T$ plane can be explored by varying the parameters of the colliding nuclei, such as energy, atomic numbers $A_{1}, A_{2}$, impact parameter. We show an example by the full line, and we show only the path corresponding to the cooling of the plasma, i.e., the time evolution after the termination of the nuclear collision, assuming a plasma formation. At the phase boundary described qualitatively by the heavy dots in fig. 1.3, the baryon density $\nu$ must be discontinuous, as suggested tacitly by the first order phase transition shown in fig. 1.2. This, and other theoretical reasons make the use of the baryochemical potential $\mu_{\mathrm{b}}$ instead of $\rho_{\mathrm{b}}$, more advantageous since $\mu_{\mathrm{b}}$, like $T$, is continuous at a phase transition (assuming the presence of a thermodynamical bath). In general, $\rho_{\mathrm{b}}$ and $\mu_{\mathrm{b}}$ may, at any given $T$, be related to each other, if enough is known about the structure of a physical system. But even more importantly, $\mu_{\mathrm{b}}$ like $T$, is a measurable thermodynamic quantity and we will get more used to it in the remainder of this paper. At this time it is first necessary to get a semiquantitative idea which values of $T$ and $\mu_{\mathrm{b}}$ are limiting the existence of the quark-gluon plasma. In fig. 1.4 several boundaries between the phases are shown. The hadronic gas region encompasses the point $\mu_{\mathrm{b}}=T=0$, while quark-gluon plasma is found for $T>T_{\mathrm{cr}}\left(\mu_{\mathrm{b}}\right)$. A first estimate concerning the boundary between the phases in the $\mu-T$ diagram may be obtained considering the ideal gas equations of state of quarks and gluons contained by the vacuum pressure $B$ and the pressure of the hot (massless) pion gas at a given temperature, i.e.

$$
P_{\mathrm{QGP}}=\frac{37}{90} \pi^{2} T^{4}+\mu_{\mathrm{q}}^{2} T^{2}+\frac{1}{2 \pi^{2}} \mu_{\mathrm{q}}^{4}-B, \quad P_{\mathrm{HAD}}=\frac{3 \pi^{2}}{90} T^{4} .
$$

The choice $B^{1 / 4}=150 \mathrm{MeV}$ (near the MIT bag value) leads to the curve marked I in fig. 1.4, while $B^{1 / 4}=250$ leads to the curve marked II. Curve III is the statistical bootstrap critical boundary with a chosen critical point $T_{\text {cr }}=T_{0}=190 \mathrm{MeV}$ at $\mu_{\mathrm{b}}=0$ [HR80].

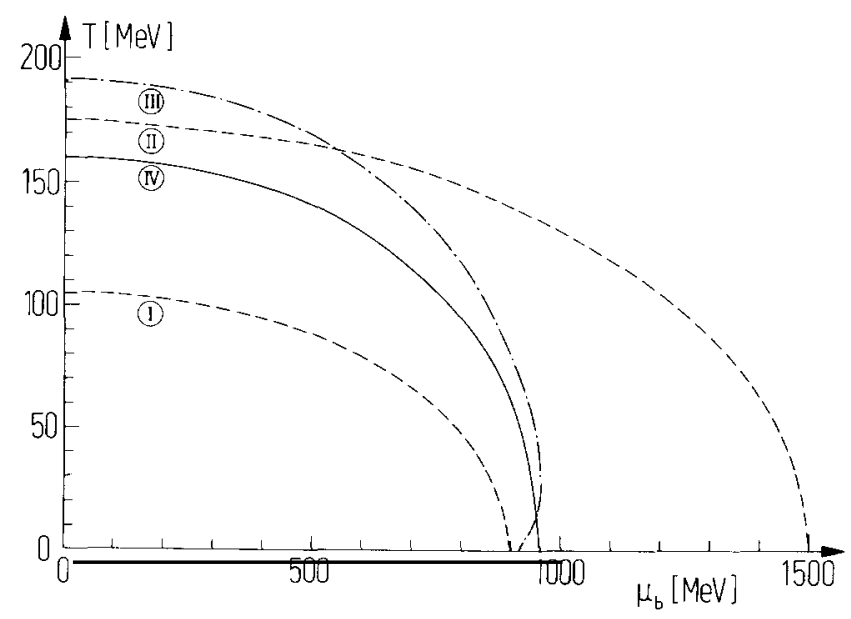

Fig. 1.4. Semiquantitative phase boundaries between quark-gluon plasma and hadronic gas phases. The curve marked IV is used in the domain $0<\mu_{\mathrm{b}}<600 \mathrm{MeV}$ in this paper. See text after eq. (1.16) for explanation of details. 
Table 1.1

Selected critical values of $\left(\mu_{\mathrm{cr}}, T_{\mathrm{cr}}\right)$ and the corresponding $\mu_{\mathrm{b}} / T$ ratio. For $T_{0}=250 \mathrm{MeV}$ and $V \sim t$ as well as $-t^{3}$ we also show $t_{c}$, the lifetime of the pure quark-gluon plasma, assuming that the initial radius of the plasma is $\underline{3} \underline{\mathrm{fm}}$ (see section $\underline{1.5}$ for details of assumptions)

\begin{tabular}{cllll}
\hline $\begin{array}{l}\mu_{\mathrm{b}}^{\mathrm{cr}} \\
(\mathrm{MeV})\end{array}$ & $\begin{array}{l}T_{\mathrm{cr}} \\
(\mathrm{MeV})\end{array}$ & $\mu_{\mathrm{b}} / T$ & $\begin{array}{l}t_{\mathrm{c}}^{(1)} \\
(\mathrm{fm} / \mathrm{c})\end{array}$ & $\begin{array}{l}t_{\mathrm{c}}^{(3)} \\
(\mathrm{fm} / \mathrm{c})\end{array}$ \\
\hline 0 & 160 & 0 & 17 & 7.0 \\
200 & 156 & 1.3 & 18 & 7.2 \\
400 & 148 & 2.7 & 22 & 7.6 \\
600 & 130 & 4.6 & 32 & 8.7 \\
\hline
\end{tabular}

The boundary curve marked IV (full line) will be used in our calculations. It is selected rather arbitrarily by fixing $B^{1 / 4}=200 \mathrm{MeV}, T_{\mathrm{cr}}\left(\mu_{\mathrm{b}}=0\right)=160 \mathrm{MeV}$ and $\mu_{\mathrm{cr}}(T=0)=960 \mathrm{MeV}$ and interpolating according to eq. (1.16) quadratically in between. Note, however, that the coefficients of the quadratic form, LHS of eq. (1.16) were not taken as given by the free quark-gluon gas, but are determined by the above choice of parameters. Explicitly the boundary selected by us satisfies the equation:

$$
\left(T / T_{\mathrm{cr}}^{0}\right)^{4}+\left(T / T_{\mathrm{cr}}^{0}\right)^{2}\left(\mu_{\mathrm{q}} / \mu_{\mathrm{qcr}}^{0}\right)^{2}\left(\mu_{\mathrm{qcr}}^{0} / B^{1 / 4}\right)^{2}\left(T_{\mathrm{cr}}^{0} / B^{1 / 4}\right)^{2}+\left(\mu_{\mathrm{q}} / \mu_{\mathrm{qcr}}^{0}\right)^{4}=1 .
$$

The motivation to choose $T_{\mathrm{cr}}=160 \mathrm{MeV}$ resides in the remarkable fact that transverse momentum spectra in hadronic reactions are governed by a universal slope parameter of $160 \mathrm{MeV}$ [Ha65, HR68]. $\mu_{\mathrm{cr}}=960 \mathrm{MeV}$ was chosen quite arbitrarily in order for $\mu_{\mathrm{cr}}(T=0)$ to be near curve I (MIT bag) and curve III (bootstrap model) at moderate temperatures. However unrealistic this latter choice may be, it will be of no influence in the following considerations where only $\mu_{\mathrm{b}}<600 \mathrm{MeV}$ is used. Selecting a number of values of $\mu_{\mathrm{b}}$ along the critical curve in fig. 1.4 we find the corresponding values of critical temperature and $\mu / T$ as shown in table 1.1 .

\subsection{Evolution for quark-gluon plasma}

\subsubsection{Baryon-rich plasma}

We must make a further assumption in order to describe the physical phenomena and the time evolution of the quark-gluon plasma in nuclear collisions. Our first reaction picture may be realized in two ways. Either it implies stopped nuclear matter leading to a baryon-rich environment for the plasma with relatively large matter radius $R_{0}$ [DR82] or it may be realized in the fragmentation region at ultra-high energies beyond the stopping regime [CDH83]. In the first case assume that the volume occupied by the plasma grows rapidly with time as more nuclear matter enters the reaction zone, while the temperature is assumed to be nearly constant. After a characteristic fireball formation time $t_{0}$ the inflow of matter ceases, and due to continuing expansion the temperature $T$ begins to decrease faster such that $V T^{3}$ ( entropy) is a constant in time for $t>t_{0}$. In the second case $t_{0}$ corresponds to the proper collision time of the penetrating nuclei, measured in the rest frame of the nucleus.

We have therefore the following model assumptions:

$$
V=V_{0}\left(t / t_{0}\right)^{n}, \quad t>t_{0}
$$




$$
T= \begin{cases}T_{0}\left(t / t_{0}\right)^{-n / 3}, & t>t_{0} \\ T_{0}, & t<t_{0} .\end{cases}
$$

$n$ is a parameter, to be taken below as either one or three, to describe longitudinal and spherical expansion, respectively, and the plasma time constant is assumed to be linearly dependent on the plasma radius $R_{0}$,

$$
t_{0}=1.5 R_{0}
$$

as suggested by some hydrodynamical calculations [Ch82]. Equation (1.18b) implies further that the time $t_{\mathrm{c}}$ required for the initial plasma temperature $T_{0}$ to drop to the critical temperature $T_{\mathrm{c}}$ is

$$
t_{\mathrm{c}}=1.5 R_{0}\left(T_{0} / T_{\mathrm{c}}\right)^{3 / n}
$$

The magnitude of $R_{0}$ considered by us is in the domain of $2-4 \mathrm{fm} ; T_{0}$, the initial plasma temperature, will be in the interval $T_{\mathrm{c}}<T_{0}<250 \mathrm{MeV}$ where, as per assumption, $T_{\mathrm{c}}(\mu=0)=160 \mathrm{MeV}$. We note that for $R_{0}=2 \mathrm{fm}$ (i.e. $t_{0}=3 \mathrm{fm} / c=10^{-23} \mathrm{~s}$ ) and $T_{0}=200 \mathrm{MeV}$, eq. (1.19) with $n=1$ leads to $t_{\mathrm{c}}=6 \mathrm{fm} / c=$ $2 \times 10^{-23} \mathrm{~s}$, a 'canonical' time used quite often further below. In the other extreme, with $T_{0}=250 \mathrm{MeV}$ and $R_{0}=4 \mathrm{fm}$, one finds 3 times longer baryon lifetime, $t_{\mathrm{c}}=18 \mathrm{fm} / c$. However, for $n=3$ we find in this case $8.6 \mathrm{fm} / c$. In fig. 1.5 we show schematically the time evolution of $V$ and $T$. Of course, this simple parameterization presented here of the plasma evolution is quite inadequate for a more detailed study. However, its simplicity provides for a first insight into the role that dynamics of nucler collisions and plasma formation and evolution is going to play in the study of properties of this new form of matter by observation of strange antibaryons.

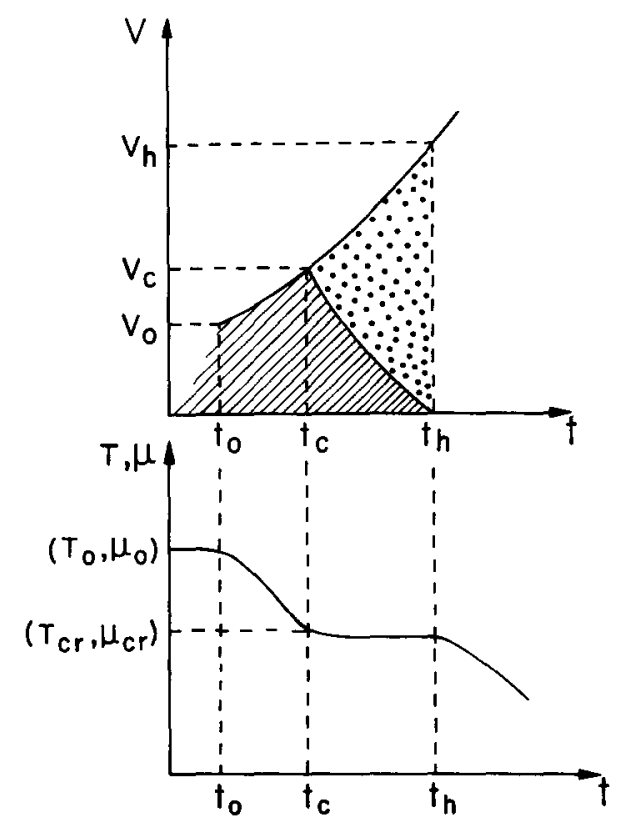

Fig. 1.5. Schematic representation of time evolution of the reaction volume and temperature. Dotted is the hadronic phase. For $t<t_{\mathrm{c}}$ pure quark-gluon plasma is present. $t_{0}$ is the plasma formation time. 
A further refinement of the time evaluation of the plasma state is necessary whenever we need to consider the light quark content, which is controlled by the baryochemical potential. The simple assumption

$$
\mu_{\mathrm{b}}(t) / T(t) \approx \text { const. }
$$

appears to be the best choice in our context. This leads to

$$
\mu_{\mathrm{b}}(t)= \begin{cases}\mu_{\mathrm{b}}^{0}\left(t / t_{0}\right)^{-n / 3}, & t>t_{0} \\ \mu_{\mathrm{b}}^{0}, & t<t_{0} .\end{cases}
$$

The actual value of $\mu_{\mathrm{b}} / T$ established in a particular collision of two nuclei depends on the yet not well-known stopping power of nuclear matter as well as on the parameters of the collision: kinetic energy, size of projectile and target, impact parameter. In the last columns of table 1.1 we give the expected lifetime of the pure plasma state that is the time $t_{\mathrm{c}}$ until the critical values $\mu_{\mathrm{c}}, T_{\mathrm{c}}$ are reached, assuming $R_{0}=3 \mathrm{fm}, t_{0}=1.5 R_{0}$.

As a consequence of the assumption (1.20) the entropy per baryon in the plasma remains constant. However, the baryon number increases for $t<t_{0}$, due to growth of the volume of the plasma state. For $t>t_{0}$ the baryon number remains constant and so does the total entropy of the plasma state. The thermal energy per baryon is constant for $t<t_{0}$ and it is decreasing for $t>t_{0}$. The consequences of this dynamical model for strange particle evolution and formation will be described in some detail in section 7.

\subsubsection{Baryon-symmetric ( gluonic) plasma}

At ultra-high collision energies, when the nuclei may not be able to stop each other, a quark-gluon plasma with vanishing baryon number density is expected to develop in the central rapidity region [AKM80]. The space-time development of this gluonic matter is described by the appropriately modified hydrodynamical model of Landau for hadronic collisions at very high energy [Bj83, Ba83, KRR83]. The matter expands mainly longitudinally, but there is also some flow in the transverse directions.

In the local restframe, the variation of the energy density $\varepsilon$ with proper time is related to the divergence of the local four-velocity $u^{\mu}$ :

$$
\frac{\mathrm{d}}{\mathrm{d} \tau}(\ln \varepsilon)=-\left(1+v_{\mathrm{s}}^{2}\right) \partial_{\mu} u^{\mu}
$$

where $v_{\mathrm{s}}$ is the velocity of sound in the quark-gluon plasma $\left(1 / \sqrt{3}=v_{\mathrm{s}}\right.$ for an ultra-relativistic gas). In the framework of the inside-outside cascade model it is assumed that the partons are produced at proper time $\tau_{0} \sim 1 \mathrm{fm} / c$ at rapidity $y=\operatorname{arctanh}\left(v_{\|}\right)$, where $v$ is the longitudinal velocity in the laboratory frame. The four-velocity is then (see fig. 1.6)

$$
u^{\mu} \sim\left(\gamma, \gamma v_{\|}, 0,0\right)=(t / \tau, x / \tau, 0,0)
$$

so that $\partial_{\mu} u^{\mu}=\tau^{-1}$. The energy density therefore evolves according to the law

$$
\varepsilon(\tau)=\varepsilon_{0}\left(\tau_{0} / \tau\right)^{1+v_{s}^{2}}=\varepsilon_{0}\left(\tau_{0} / \tau\right)^{4 / 3}
$$




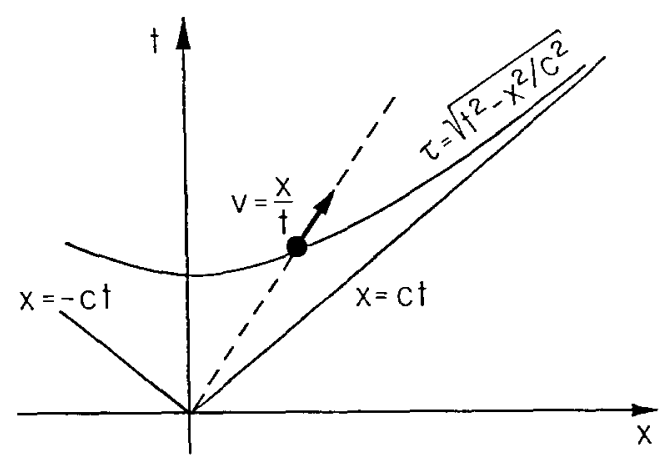

Fig. 1.6. Definition of the coordinates for the evolution of the quark-gluon plasma in the central rapidity region. The dashed line represents the curved line of a parton produced at rapidity $Y$.

As the energy density of a baryon number-symmetric plasma is strictly proportional to $T^{4}$ (see eqs. (1.4) and (1.13)) this implies that the temperature varies like in (1.18b) with $n=1$ :

$$
T=T_{0}\left(\tau / \tau_{0}\right)^{-1 / 3}, \quad \tau \geq \tau_{0},
$$

as it is appropriate for longitudinal expansion.

Having outlined the framework for a detailed study of strange hadrons emerging from the quark-gluon plasma we can now turn to the development of the subject matter by first considering what the spectrum of strange hadrons is telling us about the intrinsic properties of strange quarks.

\section{Strange quark mass and hadronic spectrum}

The parameter controlling decisively the considerations of this paper is the strange quark mass. Recently it has been shown that the choice of a running value for the strange quark mass parameter

$$
m_{\mathrm{s}}=m_{0} \ln \left(R \Lambda_{\mathrm{R}}\right)
$$

allows for an accurate description of the spectrum of strange hadrons in the framework of the MIT-bag model. $\Lambda_{\mathrm{R}}$ introduced in eq. $(2.1)$ is a new parameter to be added to the set of bag model parameters and $R$ is the confinement radius for quarks. Relation (2.1) is an empirical consequence of a systematic study of possible fits to hadronic spectra. Although eq. (2.1) is very suggestive of the continuum $Q C D$ running quark mass formula [NW81, Po76, GP76], one should not necessarily take this as evidence for the validity of $Q C D$, especially as the same study revealed that it is perfectly sufficient to use a constant value of the QCD coupling constant $\alpha_{\mathrm{s}}$. Also note that perturbative QCD predicts a power $4 / 9$ for the logarithm in eq. (2.1) [NW81, GL82].

\subsection{Phenomenological mass formula}

We now describe how the relation (2.1) is extracted from the empirical knowledge about the hadronic spectrum [AR84, AR85]. Consider the MIT bag Hamiltonian [deG75, Jo75]: 


$$
H=B \frac{4}{3} \pi R^{3}+\frac{z_{0}}{R}+\sum_{i} \frac{n_{i} \varepsilon_{i}}{R}+\frac{1}{R} \sum_{i \geq j} c_{i j} h_{i j}
$$

Here $n_{i}$ counts the number of quarks with flavour $i \in\{\mathrm{u}, \mathrm{d}, \mathrm{s}\}$. Isospin breaking effects are not included in eq. (2.2). The coefficients $c_{i j}$

$$
c_{i j}=\left\langle\mathrm{h}\left|\sum_{k<1}(F \sigma)_{i_{k}}(F \sigma)_{j_{l}}\right| \mathrm{h}\right\rangle,
$$

with $F$ standing for the generators of colour SU(3) and $\sigma$ denoting the spin matrices $C_{i j}$ are the expectation values of the colour hyperfine interaction. There are thus 7 parameters: $B, z_{0}, \varepsilon_{\mathrm{q}}, \varepsilon_{\mathrm{s}}, h_{\mathrm{qq}}$, $h_{\mathrm{qs}}$ and $h_{\mathrm{ss}}$ of which the last 4 can be functions of $m_{\mathrm{s}} R$. We recall that the quark energies (in units of $1 / R)$, are usually determined from boundary conditions imposed at the confinement radius $R$. This normally fixes the ratios $h_{\mathrm{qq}} / h_{\mathrm{qs}} / h_{\mathrm{ss}}$, as well as $R$-dependence of $h_{\mathrm{qs}}$ and $h_{\mathrm{ss}}$. By allowing all these matrix elements and energy eigenvalues to assume slightly different values than expected in a model framework, one can account for small, but significant higher order effects, while avoiding the introduction of ad hoc assumptions as to the quark dynamics in the bag and at the surface, as they are introduced, e.g., in chiral bag models.

The nonstrange quarks are assumed to be approximately massless while the strange quarks have a nonnegligible mass. The parameters describing the properties of the strange quarks will depend in a significant manner on $m_{\mathrm{s}} R$, a dimensionless quantity. It is more straightforward to avoid any explicit mentioning of $m_{\mathrm{s}}$ and write a parametric representation:

$$
\begin{aligned}
& \varepsilon_{\mathrm{s}}(R)=\varepsilon_{\mathrm{q}}+\varepsilon_{\mathrm{s}}^{(1)} R+\varepsilon_{\mathrm{s}}^{(2)} R^{2}+\cdots \\
& h_{\mathrm{qs}}\left(M_{\mathrm{s}} R\right)=h_{\mathrm{qs}}(0)+h_{\mathrm{qs}}^{(1)} R+\cdots \\
& h_{\mathrm{ss}}\left(M_{\mathrm{s}} R\right)=h_{\mathrm{ss}}(0)+h_{\mathrm{ss}}^{(1)} R+\cdots
\end{aligned}
$$

where $\varepsilon^{(1)}, \varepsilon^{(2)}, h_{\mathrm{qs}}^{(1)}, h_{\mathrm{ss}}^{(1)}$ may be viewed as further initially independent and dimensioned parameters to be fixed by comparison with the spectrum of strange hadrons. Fitting all the parameters by considering the hadronic spectrum, the $R$-dependence of $\varepsilon_{\mathrm{s}}$, the strange quark energy, is determined. However, as is apparent from eq. (2.4a), one cannot, without a further assumption or physical consideration, identify a numerical value for the mass or kinetic energy of the strange quarks - initially only the strange quark energy, $\varepsilon_{\mathrm{s}}(R) / R$, will be extracted.

\subsection{Fitting procedures}

Explicit fitting procedures show that it is sufficient to consider only the second order terms in $R$ in eq. (2.4a) and first order terms in eqs. (2.4b) and (2.4c). At this point it is worthwhile to realise that each such hypothesis leading to a reduction of the number of parameters can be easily verified numerically by adding or omitting parameters. Failure of improvement of a fit of the hadronic spectrum with an additional parameter implies that it is not necessary to consider such a refinement. In that way it is found that $\varepsilon^{(1)}=0$ and further that the hyperfine interaction is arising from two-body forces since the relationship $h_{\mathrm{ss}}^{(1)}=2 h_{\mathrm{qs}}^{(1)}$ is confirmed in actual numerical fits. 
Table 2.1

Upper portion and lower portion: stable and unstable hadrons. First column: Experimental isospin average masses used as inputs in least square fits. Fit A: Empirical formula for strange quark energy. Fit B: Running strange quark mass

\begin{tabular}{lccc}
\hline Particle & Exp. mass & Fit A & Fit B \\
\hline \hline $\mathrm{N}$ & 939 & 940 & 938.8 \\
$\omega$ & 783 & 782 & 783.1 \\
$\mathrm{~K}$ & 496 & 496 & 495.9 \\
$\phi$ & 1020 & 1021 & 1020.1 \\
$\Sigma$ & 1193 & 1193 & 1193.5 \\
$\Lambda$ & 1116 & 1115 & 1115.6 \\
$\Xi$ & 1318 & 1318 & 1318.3 \\
$\Omega$ & 1672 & 1672 & 1671.8 \\
\hline$\Delta$ & 1232 & 1240 & 1234 \\
$\mathrm{~K}^{*}$ & 895 & 907 & 908.8 \\
$\Sigma^{*}$ & 1385 & 1394 & 1391.4 \\
$\Xi$ & 1533 & 1537 & 1537.1 \\
\hline
\end{tabular}

(A) $E_{\mathrm{s}}=\varepsilon_{\mathrm{q}} / R+\varepsilon^{(2)} R$

(B) $m_{\mathrm{s}}=234 \mathrm{MeV} \ln (R \cdot 760 \mathrm{MeV}), B^{1 / 4}=145.5 \mathrm{MeV}$.

With the six remaining parameters [i.e. $B, z_{0}, \varepsilon_{\mathrm{q}}, \varepsilon^{(2)}, h_{\mathrm{qq}}, h_{\mathrm{qs}}^{(1)}\left(h_{\mathrm{ss}}^{(1)}=2 h_{\mathrm{qs}}^{(1)}\right.$ and $\left.\varepsilon^{(1)}=0\right)$ ] a fit of the eight stable hadron masses leads to a typical spectrum given under Fit $A$ in table 2.1. The masses can be fitted on the average to within $0.7 \mathrm{MeV}$ of the assumed values, the masses of the wide states are predicted quite closely to their actual physical values, yet consistently a little too high (see [AR84] for a qualitative discussion of this behaviour).

However correlations found between the parameters in Fit A imply that there were too many parameters. This is certainly the case since $B, \varepsilon^{(2)}$ and $h_{\mathrm{qs}}^{(1)}$ are three dimensioned parameters while the physics of the problem requires at most two independent dimensioned quantities, namely those associated with the hadronic vacuum and the strange quark mass. These correlations are eliminated by fixing or dropping of certain parameters: $h_{\mathrm{qs}}^{(1)}$ is almost completely determined by the $\Sigma-\Lambda$ splitting. The fit gives $h_{\mathrm{qs}}^{(1)}=-29.3 \pm 2.4 \mathrm{MeV}$ compared to $\frac{3}{8}\left(M_{\Lambda}-M_{\Sigma}\right)=-29.2 \mathrm{MeV}$. Further, the hadronic spectrum is found to be sensitive only to the combinations $B^{1 / 3} \varepsilon_{\mathrm{q}}, B^{1 / 3} z_{0}$ and $B^{1 / 3} h_{\mathrm{qq}}$ and $\varepsilon^{(2)}$. Therefore one can choose one of the four parameters $B, z_{0}, \varepsilon_{\mathrm{q}}, h_{\mathrm{qq}}$, without affecting the fit. The choice $B^{1 / 4}=171 \mathrm{MeV}$ [AR84] leads to $\varepsilon_{\mathrm{g}}=1.688, z_{0}=-1.559$ and $h_{\mathrm{qq}}=0.320$ all values being reduced in comparison with the MIT bag fit [deG75]. Note that in the MIT bag model the value $\varepsilon_{\mathrm{q}}=2.043$ is determined from the boundary condition on the unperturbed fermion spinors. Selecting this value, i.e., fixing $\varepsilon_{\mathrm{q}}=2.043$ results in $B^{1 / 4}=147 \mathrm{MeV}, z_{0}=1.886, h_{\mathrm{qq}}=0.387$ : all values are close but slightly different from the original MIT fit. The quantity $\varepsilon^{(2)}(4 \pi B)^{-1 / 3}=0.203 \mathrm{GeV}^{2 / 3}$ is determined by the $\mathrm{N}-\Lambda$ splitting. Once $B$ is chosen at a preferred value, $\varepsilon^{(2)}$ can be extracted and the energy in each hadron is determined. The important message here is that the isospin averaged hadronic spectrum in the $u, d, s$ sector cannot fix uniquely a value of $\varepsilon_{\mathrm{q}}$ or $B$ independent of a theoretical bias even though only few parameters are fitted to a large number of hadronic states.

\subsection{Strange quark energy and mass}

Once $\varepsilon_{\mathrm{q}}$ or $B$ is selected, the energy of the strange quark $E_{\mathrm{s}}$ as function of $R$ is fixed. Although $\varepsilon_{\mathrm{s}}(R)$ 
varies with choice of $B$ (viz. $\varepsilon_{q}$ ) the energy of strange quarks in various hadrons is virtually independent of this arbitrariness, as the radius $R$ of hadrons changes accordingly. A number of fits to different functional forms $\varepsilon_{\mathrm{s}}$ is shown in fig. 2.1 , all made assuming $B^{1 / 4}=0.170 \mathrm{GeV}$ [AR85]. The point to notice here is that the strange quark energy does not depend on the method of parameterization, underpinning the physical relevance of the result. The assumed forms are:

$$
\varepsilon_{\mathrm{s}} / R=\left\{\varepsilon_{\mathrm{q}}+\varepsilon_{\mathrm{s}}^{(n)} R^{n}+\varepsilon_{\mathrm{s}}^{(m)} R^{m}\right\} / R .
$$

The different curves in fig. 2.1 were determined by assuming values $(n, m) \in(0,1,2,3,4)$ and computing the least square fit to the hadronic spectrum. All different fits terminate with a very good $\chi^{2}$, except for the linear fit $(1,0)$ - dot dashed in fig. 2.1 , which lacks the necessary ability to turn upwards at large $R$.

The energy of strange quarks, as a function of $R$, is determined by the study of the hadronic spectrum, once $B^{1 / 4}$ is selected. In fig. 2.2 we show the energy of strange quarks in various hadrons for various fits with different $B^{1 / 4}$ - recall that we can have any value of $B^{1 / 4}$ as long as $B^{1 / 3} \varepsilon_{\mathrm{q}}$ is kept constant. There is practically no variation of the strange quark energies with a different choice of $B^{1 / 4}$. The strange quark energies in hadrons all are in the range $554-594 \mathrm{MeV}$ with the exception of kaons where it is $715 \mathrm{MeV}$ due to the very small confinement radius.

The large deviation of $E_{\mathrm{s}}(R)$ from kinematic behaviour found in [AR85] suggests that a physical parameterization of the mass $m_{\mathrm{s}}$ is required, such as given in eq. (2.1). Then

$$
E_{\mathrm{s}}(R)=\varepsilon_{\mathrm{s}} / R=\sqrt{\left[\varepsilon_{\mathrm{q}} m_{\mathrm{s}}(R) / R\right]^{2}+\left(m_{\mathrm{s}}(R)\right)^{2}}
$$

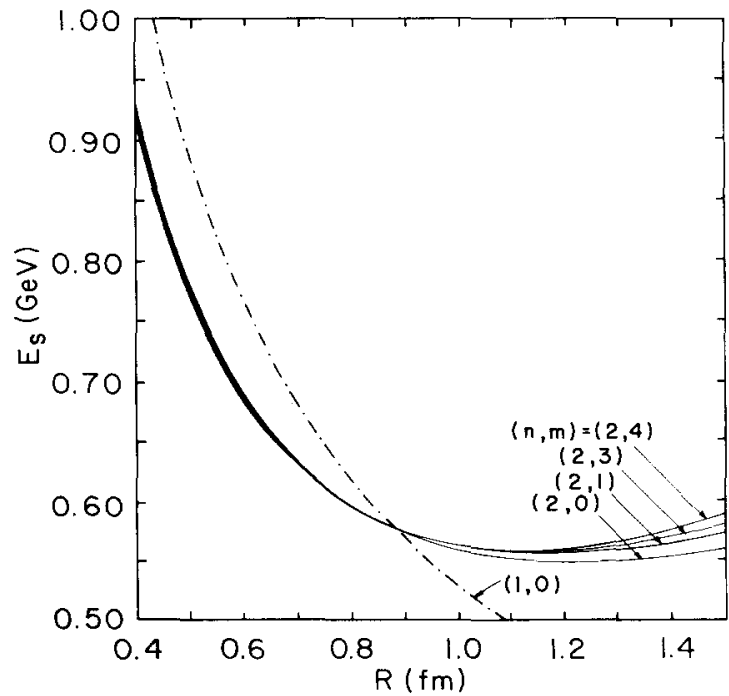

Fig. 2.1. The strange quark energy as a function of confinement radius $R$ for various parameterisations, eq. $(2.5): \mathrm{a}=(n, m)=(2.4)$ : $b=(2.3): c=(2.1): d=(2.0):$ dash dotted $=(1.0) . B^{1 / 4}=170 \mathrm{MeV}$ is assumed in these fits.

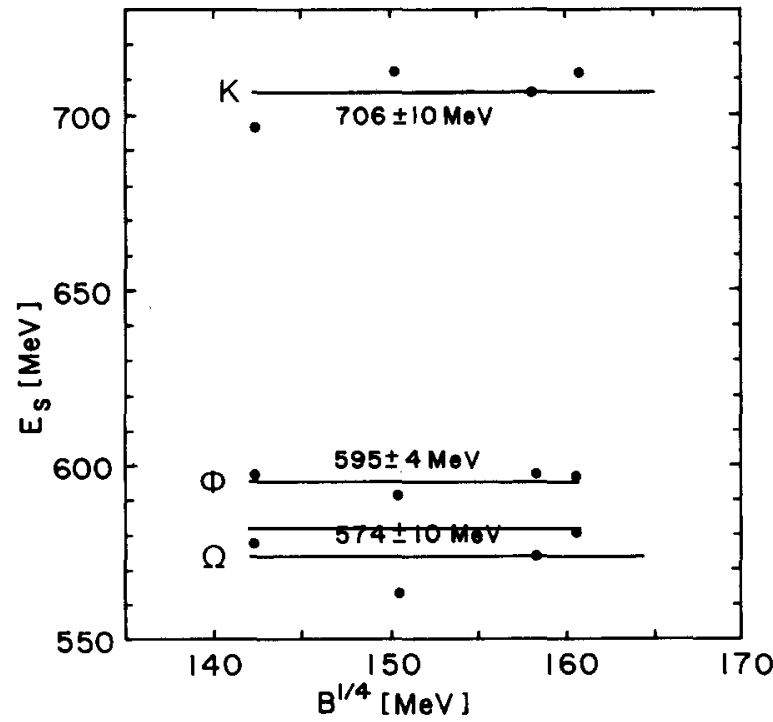

Fig. 2.2. (In)dependence of strange quark energy on $B^{1 / 4}$. 
and $m_{\mathrm{s}}(R)$ varies according to eq. (2.1) while $\varepsilon_{\mathrm{q}}$ is made $R$ dependent according to the boundary value problem posed by the MIT bag model. Assuming $B^{1 / 4}=145.5 \mathrm{MeV}$ the light quark momentum was found at $\varepsilon_{\mathrm{q}}(0)=2.049 \pm 0.013$, very near to the Dirac equation bag eigenvalue. The resulting hadronic masses are given in Fit $B$, table 2.1. All narrow hadronic masses are fitted within $0.5 \mathrm{MeV}$. The other parameters found [AR85] are $h_{\mathrm{qq}}=0.388 \pm 0.006, z_{0}=-1.85 \pm 0.025, m_{0}=234 \pm 14 \mathrm{MeV}, \Lambda_{\mathrm{R}}=760 \pm$ $57 \mathrm{MeV}$. There is a slight correlation between $\varepsilon_{\mathrm{q}}$ and $z_{0}$ and an important one between $m_{0}$ and $\Lambda_{\mathrm{R}}$ as should be expected - only two of the three dimensioned parameters $B^{1 / 4}, m_{0}$ and $\Lambda_{\mathrm{R}}$ can have physical meaning. The effective strange quark mass varies between $173 \mathrm{MeV}$ in kaons and $321 \mathrm{MeV}$ in $\Xi^{*}$ and its $R$ dependence is clearly significant. (See table 2.2.)

Several comments must be made with reference to the above results:

(a) The assumed form of the running mass, eq. (2.1), is different from the (ln) $)^{4 / 9}$ behaviour implied by QCD. It is the need for the mass to go up with increasing $R$ (decreasing momentum) which matters but not the precise functional behaviour.

(b) In our future discussion the result that the strange quark mass is $\sim 170 \mathrm{MeV}$ in a kaon plays an important role. The energy density in a kaon (last column of table 2.2 ) is $0.75 \mathrm{GeV} / \mathrm{fm}^{3}$ while in nonstrange hadrons it is $4 B=0.24 \mathrm{GeV} / \mathrm{fm}^{3}$ for $B^{1 / 4}=145.5 \mathrm{MeV}$. We thus can associate the mass of the strange quark at the $170 \mathrm{MeV}$ level with energy density which we expect at the phase transition to the plasma phase. The energy density of $0.3-0.5 \mathrm{GeV} / \mathrm{fm}^{3}$ is found for strange hadrons in which the strange quark mass comes to $260-310 \mathrm{MeV}$. Note that, in general, strange hadrons are 'denser' as there is less Fermi pressure to balance the vacuum pressure, since the massive quark is more inert than a massless one.

(c) The running strange quark mass seems to be an essential element in the understanding of the hadronic spectrum in the framework of the bag model mass formula (2.2). Its smallness at high energy density assures that strangeness will be easily made in quark-gluon plasma (see section 3 ).

Table 2.2

Masses, radii, strange quark energies, strange quark mass and energy density hadrons as fitted. $B^{1 / 4}=170 \mathrm{MeV}$ is assumed in these fits

\begin{tabular}{|c|c|c|c|c|c|}
\hline Particle & $\begin{array}{l}\text { Fitted } \\
\text { mass } \\
(\mathrm{MeV})\end{array}$ & $\begin{array}{l}R \text { at } \\
B^{1 / 4}=145.5 \mathrm{MeV} \\
(\mathrm{fm})\end{array}$ & $\begin{array}{l}E_{\mathrm{s}} \\
(\mathrm{MeV})\end{array}$ & $\begin{array}{l}m_{\mathrm{s}}(R) \\
(\mathrm{MeV})\end{array}$ & $\begin{array}{l}\varepsilon=M / V \text { at } \\
B^{1 / 4}=145.5 \mathrm{MeV} \\
\left(\mathrm{GeV} / \mathrm{fm}^{3}\right)\end{array}$ \\
\hline \multicolumn{6}{|c|}{ Strange hadrons } \\
\hline $\mathbf{K}$ & 496 & 0.543 & 715 & 173 & 0.75 \\
\hline$\phi$ & 1020 & 0.787 & 594 & 260 & 0.50 \\
\hline$\Sigma$ & 1194 & 0.920 & 566 & 296 & 0.36 \\
\hline$\Lambda$ & 1116 & 0.920 & 566 & 296 & 0.34 \\
\hline$\Xi$ & 1318 & 0.856 & 577 & 279 & 0.50 \\
\hline$\Omega$ & 1672 & 0.910 & 568 & 294 & 0.53 \\
\hline $\mathrm{K}^{*}$ & 909 & 0.857 & 577 & 280 & 0.35 \\
\hline$\Sigma^{*}$ & 1391 & 1.022 & 554 & 321 & 0.31 \\
\hline$\Xi^{*}$ & 1537 & 0.964 & 560 & 307 & 0.41 \\
\hline \multicolumn{6}{|c|}{ Nonstrange hadrons } \\
\hline $\mathrm{N}$ & 939 & 0.987 & & & 0.24 \\
\hline$\omega$ & 783 & 0.929 & & & 0.24 \\
\hline$\Delta$ & 1234 & 1.081 & & & 0.24 \\
\hline
\end{tabular}


We are thus prompted to use $m_{\mathrm{s}}=160-180 \mathrm{MeV}$ in rough accord with the studies of current strange quark mass via various other means (see [GL82, RRY85] for a general review). The value $m_{\mathrm{s}} \sim$ $280 \mathrm{MeV}$ occasionally referred to in literature arises in the context of the MIT bag model in which the significant variation of $m_{\mathrm{s}}$ with the momentum (energy density) has been neglected. Further note that almost always one finds $E_{\mathrm{s}}=560 \mathrm{MeV} \pm 10 \mathrm{MeV}$ (kaons are sole significant exception). This is due to the flatness of $E_{\mathrm{s}}(R)$ for values of $R$ obtained for strange hadrons at given $B^{1 / 4}$, see e.g., fig. 2.1 and table 2.2. This explains why additive quark models have been highly successful in the understanding of the particle properties. However, $E_{\mathrm{s}}=560 \mathrm{MeV}$ is the constituent energy (not mass) of the strange quark in hadrons.

For the study of strange quark-pair production in the following section we shall combine the above mass parameter, $m_{\mathrm{s}} \sim 170 \mathrm{MeV}$, with the value of the running coupling constant taken at the relevant (timelike) four-momentum $Q^{2}=4 m_{\mathrm{s}^{\prime}}^{2}$ Numerically this gives

$$
\alpha_{\mathrm{s}}\left(4 m_{\mathrm{s}}^{2}\right)=\frac{4 \pi}{\left(11-\frac{2}{3} N_{f}\right) \ln \left(4 m_{\mathrm{s}}^{2} / \Lambda^{2}\right)} \sim 0.6
$$

for $N_{f}=3$ flavour and $\Lambda \sim 100 \mathrm{MeV} / \mathrm{c}$ being suggested by fits to scaling violation in deep-inelastic lepton hadron scattering [SW82].

\section{Heavy quark production in quark-gluon plasma}

Consider a relativistic nuclear collision in which a transitional state of quark-gluon plasma occurs. The production of strangeness carrying hadrons proceeds through three steps: (i) locally deconfined strange quarks and antiquarks are created in the quark-gluon phase; (ii) these quarks are incorporated as hadronic constituents during the disintegration of the quark-gluon plasma; and (iii) strange hadrons continue to react, are produced and destroyed in the final hadronic gas phase by (confined) strangeness creation, annihilation or exchange reactions. In this section we study the elementary processes of strange, or more generally heavy, quark pair-production and annihilation in the quark-gluon plasma, and we investigate in depth the approach to chemical equilibrium of the strange quark abundance in the plasma phase. With our apologies, the symbol 's' is used in this section to denote 'strong' in $\alpha_{s}, \sqrt{s}$ (the kinematic variable), 'strangeness' and as a unit of time, aside from further use to denote entropy density. However, it is in our opinion always possible to identify the proper meaning in the given context in which the symbol ' $s$ ' is used.

\subsection{Quark pair production}

In lowest order in perturbative QCD, $(\mathrm{s} \overline{\mathrm{s}})$-quark pairs can be created in collisions of two gluons (fig. 3.1a-c) and by annihilation of light quark-antiquark pairs (fig. 3.1d). The latter process was studied by Biro and Zimanyi [BZ82, BZ83] who found rather long relaxation times for approach to the chemical equilibrium. They therefore questioned the previously proposed [RH80, Ra81] use of (equilibrated) strangeness as a diagnostic tool for quark-gluon plasma. However, calculations of Biro and Zimanyi ignored the role of gluons which were considered by Rafelski and Müller [RM82, KMR86]. They showed that the gluonic production rate (cf. fig. $3.1 \mathrm{a}-\mathrm{c}$ ) is dominating strangeness production and leading to equilibration times comparable to the expected plasma lifetime. Thus flow of strangeness in relativistic 

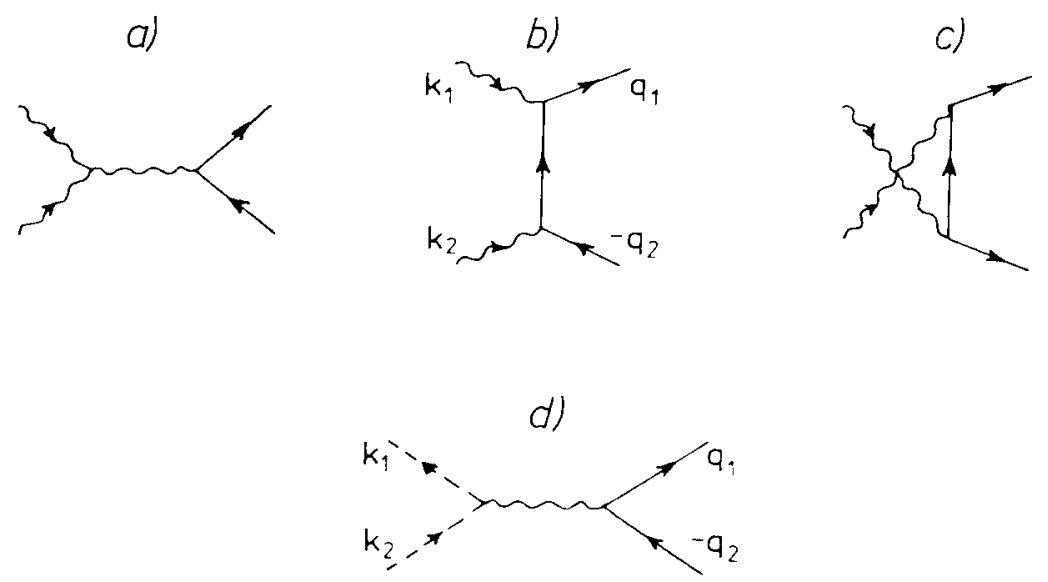

Fig. 3.1. Lowest order QCD diagrams for ss production: (a)-(c) $g g \rightarrow s \bar{s}$; (d) $q \bar{q} \rightarrow s \bar{s}$.

nuclear collisions was established as a promising method of detecting the plasma state. Because of the importance of strangeness production in this diagnostic approach to quark-gluon plasma we will rederive these results here in all detail.

The invariant matrix elements for quark-pair production have been calculated in lowest order in the QCD coupling constant $\alpha_{\mathrm{s}}$ by Georgi et al. [Ge78], Combridge [Co79] and by Matsui, Svetitsky and McLerran [Ma85]. The squared invariant matrix elements, summed over initial and final colour, spin and flavour states are for the individual diagrams shown in fig. 3.1:

$$
\begin{aligned}
& \sum\left|\mathcal{M}_{\mathrm{a}}\right|^{2}=\left(g_{\mathrm{G}} g_{\mathrm{q}} \pi \alpha_{\mathrm{s}}\right)^{2} \frac{\left(M^{2}-t\right)\left(M^{2}-u\right)}{3 s^{2}}, \\
& \sum\left|\mathcal{M}_{\mathrm{b}}\right|^{2}=\left(g_{\mathrm{G}} g_{\mathrm{q}} \pi \alpha_{\mathrm{s}}\right)^{2} \frac{2}{27} \frac{\left(M^{2}-t\right)\left(M^{2}-u\right)-2 M^{2}\left(M^{2}+t\right)}{\left(M^{2}-t\right)^{2}}, \\
& \sum\left|\mathcal{M}_{\mathrm{c}}\right|^{2}=\left(g_{\mathrm{G}} g_{\mathrm{q}} \pi \alpha_{\mathrm{s}}\right)^{2} \frac{2}{27} \frac{\left(M^{2}-t\right)\left(M^{2}-u\right)-2 M^{2}\left(M^{2}+u\right)}{\left(M^{2}-u\right)^{2}}, \\
& \sum\left|\mathcal{M}_{\mathrm{d}}\right|^{2}=N_{f}\left(g_{\mathrm{q}}^{2} \pi \alpha_{\mathrm{s}}\right)^{2} \frac{16}{81} \frac{\left(M^{2}-t\right)^{2}+\left(M^{2}-u\right)^{2}+2 M^{2} s}{s^{2}},
\end{aligned}
$$

while the interference terms between the processes $(\mathrm{a}-\mathrm{c})$ are

$$
\begin{aligned}
& \sum \mathscr{M}_{\mathrm{a}} \mathscr{M}_{\mathrm{b}}^{*}=\left(g_{\mathrm{G}} g_{\mathrm{q}} \pi \alpha_{\mathrm{s}}\right)^{2} \frac{\left(M^{2}-t\right)\left(M^{2}-u\right)+M^{2}(u-t)}{12 s\left(M^{2}-t\right)}, \\
& \sum \mathcal{M}_{\mathrm{a}} \mathscr{M}_{\mathrm{c}}^{*}=\left(g_{\mathrm{G}} g_{\mathrm{q}} \pi \alpha_{\mathrm{s}}\right)^{2} \frac{\left(M^{2}-t\right)\left(M^{2}-u\right)+M^{2}(u-t)}{12 s\left(M^{2}-u\right)}, \\
& \sum \mathscr{M}_{\mathrm{b}} \mathscr{M}_{\mathrm{c}}^{*}=\left(g_{\mathrm{G}} g_{\mathrm{q}} \pi \alpha_{\mathrm{s}}\right)^{2} \frac{M^{2}\left(s-4 M^{2}\right)}{108\left(M^{2}-u\right)\left(M^{2}-t\right)} .
\end{aligned}
$$


Here $M$ is the strange quark mass [we write $M$ instead of $m_{\mathrm{s}}$ to render the generality of the result obvious], and $s, t, u$ are the Mandelstam variables

$$
s=\left(k_{1}+k_{2}\right)^{2}, \quad t=\left(k_{1}-q_{1}\right)^{2}, \quad u=\left(k_{1}-q_{2}\right)^{2} .
$$

The momenta $k_{i}$ and $q_{i}$ are defined in fig. 3.1. The degeneracy factors for gluons and quarks are:

$$
g_{\mathrm{G}}=28_{\mathrm{s}}=16, \quad g_{\mathrm{q}}=23_{\mathrm{c}}=6,
$$

and $N_{f}=2$ is the number of light quark flavours contributing to the reaction (3.1d).

Inserting these numbers and dividing by the number of initial states, i.e. $g_{\mathrm{G}}^{2}$ for processes $(3.1 \mathrm{a}-\mathrm{c})$ and $N_{f} g_{\mathrm{q}}^{2}$ for process $(3.1 \mathrm{~d})$, we obtain the following averaged matrix elements for strange quark production by light quarks and gluons, respectively:

$$
\begin{aligned}
\sum\left|\bar{M}_{\mathrm{q} \overline{\mathrm{q}} \rightarrow \mathrm{s} \bar{s}}\right|^{2}= & \frac{64}{9} \pi^{2} \frac{\alpha_{\mathrm{s}}^{2}\left(Q^{2}\right)}{s^{2}}\left[\left(M^{2}-t\right)^{2}+\left(M^{2}-u\right)^{2}+2 M^{2} s\right] \\
\sum\left|\bar{M}_{\mathrm{gg} \rightarrow \mathrm{s} \bar{s}}\right|^{2}= & \pi^{2} \alpha_{\mathrm{s}}^{2}\left(Q^{2}\right)\left[\frac{12}{s^{2}}\left(M^{2}-t\right)\left(M^{2}-u\right)+\frac{8}{3}\left(\frac{M^{2}-u}{M^{2} t}+\frac{M^{2}-t}{M^{2}-u}\right)\right. \\
& -\frac{16}{3} M^{2}\left(\frac{M^{2}+t}{\left(M^{2}-t\right)^{2}}+\frac{M^{2}+u}{\left(M^{2}-u\right)^{2}}\right)-\frac{6}{s}\left(2 M^{2}-t-u\right) \\
& \left.+6 \frac{M^{2}(t-u)^{2}}{s\left(M^{2}-t\right)\left(M^{2}-u\right)}-\frac{2}{3} \frac{M^{2}\left(s-4 M^{2}\right)}{\left(M^{2}-t\right)\left(M^{2}-u\right)}\right] .
\end{aligned}
$$

The strong coupling constant $\alpha_{\mathrm{s}}\left(Q^{2}\right)$ here must be taken at an invariant momentum $Q^{2}$ which is most appropriately chosen as $Q^{2}=s$ for the reactions under consideration [Co79]. Note that the choice of a common $Q^{2}$ in the various diagrams contributing to the process $g \mathrm{~g} \rightarrow \mathrm{s} \overline{\mathrm{s}}$ is essential for the conservation of gauge invariance at the level of a power series expansion in $\alpha_{\mathrm{s}}$. As this choice for $Q^{2}$ is independent of the squared momentum transfer $t$, the averaged cross sections may be easily determined by integrating over the allowed range of $t$ :

$$
\bar{\sigma}=\frac{1}{16 \pi s^{2}} \int_{t_{-}}^{t_{+}} \mathrm{d} t \sum|\bar{M}|^{2}
$$

with the bounds

$$
t_{ \pm}=M^{2}-\frac{1}{2} s(1 \mp W(s))
$$

where

$$
W(s):=\left(1-4 M^{2} / s\right)^{1 / 2} .
$$

Thus we find: 


$$
\begin{aligned}
& \bar{\sigma}_{\mathrm{q} \overline{\mathrm{q}} \rightarrow \mathrm{s} \overline{\mathrm{s}}}=\frac{8 \pi \alpha_{\mathrm{s}}^{2}}{27 s}\left(1+\frac{2 M^{2}}{s}\right) W(s) \\
& \bar{\sigma}_{\mathrm{gg} \rightarrow \mathrm{s} \overline{\mathrm{s}}}=\frac{2 \pi \alpha_{\mathrm{s}}^{2}}{3 s}\left\{\left[1+\frac{4 M^{2}}{s}+\frac{M^{4}}{s^{2}}\right] \tanh ^{-1} W(s)-\left[\frac{7}{8}+\frac{31}{8} \frac{M^{2}}{s}\right] W(s)\right\} .
\end{aligned}
$$

Given the averaged cross sections we can now calculate the rate of events per unit time summed over all final and initial states. Neglecting at present final state interactions of the reaction due to the presence of strange quarks produced earlier (we return to the question of the influence of Pauli blocking in subsection 3.4) the strangeness production rate is:

$$
\frac{\mathrm{d} N}{\mathrm{~d} t}=\int \mathrm{d}^{3} x \int \frac{\mathrm{d}^{3} k_{1}}{(2 \pi)^{3}} \sum_{i} \rho_{i}\left(k_{1}, x\right) \int \frac{\mathrm{d}^{3} k_{2}}{(2 \pi)^{3}} \sum_{j} \rho_{j}\left(k_{2}, x\right) \int_{4 m_{\mathrm{s}}^{2}}^{\infty} \mathrm{d} s \delta\left(s-\left(k_{1}+k_{2}\right)^{2}\right) \bar{\sigma}(s) v_{12} .
$$

The sum over the initial states includes the discrete quantum numbers $i, j$ (colour, spin, etc.) over which the matrix elements (eq. (3.1)) were averaged. $v_{12}=\left(k_{1} \cdot k_{2}\right) /\left|k_{1}\right|\left|k_{2}\right|$ is the relative velocity of the initial particles, which are taken as massless. In order to facilitate the calculations, a dummy integration over $s$ has been introduced.

Next, we have to specify the phase-space densities $\rho_{i}(k, x)$ for light quarks and gluons in the initial state. In the circumstance of a nuclear collision these will initially be given by the Lorentz boosted quark and gluon structure functions of the colliding nuclei. During the collision these will be modified due to parton-parton collisions and become, under circumstances favourable to the formation of quark-gluon plasma, the thermal distribution functions, boosted to the appropriate rapidity along the beam direction. As was argued on the basis of perturbative QCD [vHP75, CKR77], the $\mathrm{gg} \rightarrow \mathrm{gg}$ scattering process has a large cross section due to the large number of colour degrees of freedom resulting in a short mean free path for gluons. Furthermore, the anticipated lifetime of the fireball created in an ultrarelativistic nuclear collision is of order $6 \mathrm{fm}=2 \times 10^{-23} \mathrm{~s}$, accompanied by an energy density above $1 \mathrm{GeV} / \mathrm{fm}^{3}$. Under these conditions it is likely that each perturbative quantum (light quark, gluon) will rescatter many times during the lifetime of the plasma. One should recall here as well that randomization of the particle momenta leading to a Maxwellian distribution needs under such circumstances only few collisions. Hence, we approximate the momentum distribution functions by the statistical Bose, or respectively, Fermi distribution functions which are (cf. section 1.3)

$$
\begin{aligned}
& f_{\mathrm{g}}(k)=(\exp (\beta \cdot k)-1)^{-1} \\
& f_{\mathrm{q}}(k)=\left(\exp \left(\beta \cdot k-\mu_{\mathrm{q}} / T\right)+1\right)^{-1} \\
& f_{\overline{\mathrm{q}}}(k)=\left(\exp \left(\beta \cdot k+\mu_{\mathrm{q}} / T\right)+1\right)^{-1}
\end{aligned}
$$

where $\beta \cdot k=\beta_{\mu} k^{\mu}$ and $\left(\beta_{\mu} \beta^{\mu}\right)^{-1 / 2}=T$ is the temperature in the local rest frame. In the rest frame of the plasma we have simply $\beta \cdot k=|k| / T$, as light quarks and gluons are considered to be massless.

The equilibrium distributions (3.10) can only be taken seriously for $|\boldsymbol{k}|$ not very much larger than $T$; to populate the high energy tail of the distributions many parton-parton collisions are required, for which there may not be enough time during the lifetime of the plasma. This remark is of particular 
relevance concerning the production of quarks of heavier flavour, such as charmed quarks from the thermalized plasma state. For $m_{\mathrm{c}} \sim 1.5 \mathrm{GeV}$ we have $m_{\mathrm{c}} / T \sim 7-10$ and, therefore, the representation of the parton distribution functions by the expressions (3.10) is inappropriate there. We also note that, while in each individual nuclear collision the momentum distribution may vary, in the ensemble of many different collisions the statistical distributions may be a good approximation of the circumstances. We further note that by considering only the equilibrated phase-space distributions (3.10) in which particles have relatively small randomised momentum, we implicitly neglect pre-equilibrium production of strange quark pairs from direct collisions of quarks and gluons before colliding nuclei form the plasma state, i.e. the direct production of strange hadrons through direct nucleon-nucleon collisions in the early stage of the reaction. However, as strange quark-pair production is not kinematically suppressed in the quark-gluon plasma phase, contrary to the nuclear phase as we shall show below, we do not anticipate that the pre-equilibrium production mechanism contributes significantly to the strangeness population. However, one may expect it to dominate the production of heavy flavours, e.g. charmed quarks (see section 3.5).

In view of these qualifying remarks, we now proceed using in eq. (3.9) for the phase space densities $\rho_{i}(k, x)$ the statistical momentum distributions $f_{\mathrm{g}}(k), f_{\mathrm{q}}(k), f_{\overline{\mathrm{q}}}(k)$ of gluons, quarks and antiquarks which can still have a parametric space-time dependence, i.e. through $T(x)$ or $\mu_{\mathrm{q}}(x)$. The invariant strangeness formation rate per unit time and volume for the elementary processes shown in fig. 3.1 is then:

$$
\begin{aligned}
A= & \frac{\mathrm{d} N}{\mathrm{~d} t \mathrm{~d}^{3} x}=\frac{1}{2} \int_{4 M^{2}}^{\infty} s \mathrm{~d} s \delta\left(s-\left(k_{1}+k_{2}\right)^{2}\right) \int \frac{\mathrm{d}^{3} k_{1}}{(2 \pi)^{3}\left|k_{1}\right|} \int \frac{\mathrm{d}^{3} k_{1}}{(2 \pi)^{3}\left|k_{2}\right|} \\
& \cdot\left\{\frac{1}{2} g_{\mathrm{G}}^{2} f_{\mathrm{g}}\left(k_{1}\right) f_{\mathrm{g}}\left(k_{2}\right) \bar{\sigma}_{\mathrm{gg} \rightarrow \mathrm{s} \overline{\mathrm{s}}}(s)+N_{f} g_{\mathrm{q}}^{2} f_{\mathrm{q}}\left(k_{1}\right) f_{\overline{\mathrm{q}}}\left(k_{2}\right) \bar{\sigma}_{\mathrm{q} \overline{\mathrm{q}} \rightarrow \mathrm{s} \overline{\mathrm{s}}}(s)\right\} .
\end{aligned}
$$

The factor $1 / 2$ in front of the phase space density of gluons accounts for the fact that the reaction is proportional to the number of gluon pairs in each volume element, and the double integration over $k_{1}$ and $k_{2}$ counts each gluon pair twice. This factor is missing in a number of previous computations [RM82, BZ83, CP84, MM84] and is introduced in [Ma85, KMR86]. As we have implicitly assumed that in the rest frame of the plasma the distribution functions $f$ only depend on the absolute value of the momentum, $|\boldsymbol{k}|=k_{0} \equiv k$, we can evaluate the angular integrals in eq. (3.11):

$$
\begin{aligned}
A= & \frac{4}{\pi^{4}} \int_{4 M^{2}}^{\infty} s \mathrm{~d} s \bar{\sigma}_{\mathrm{gg} \rightarrow \mathrm{s} \bar{s}}\left[\int_{0}^{\infty} \mathrm{d} k_{1} \int_{0}^{\infty} \mathrm{d} k_{2} \theta\left(4 k_{1} k_{2}-s\right) f_{\mathrm{g}}\left(k_{1}\right) f_{\mathrm{g}}\left(k_{2}\right)\right] \\
& +\frac{9}{4 \pi^{4}} \int_{4 M^{2}}^{\infty} s \mathrm{~d} s \bar{\sigma}_{\mathrm{q} \overline{\mathrm{q}} \rightarrow \mathrm{s} \bar{s}}\left[\int_{0}^{\infty} \mathrm{d} k_{1} \int_{0}^{\infty} \mathrm{d} k_{2} \theta\left(4 k_{1} k_{2}-s\right) f_{\mathrm{q}}\left(k_{1}\right) f_{\overline{\mathrm{q}}}\left(k_{2}\right)\right],
\end{aligned}
$$

where the step function requires that $k_{1} k_{2} \geq s / 4 \geq M^{2}$, and the functions $f_{\mathrm{g}}(k), f_{\mathrm{q}}(k), f_{\overline{\mathrm{q}}}(k)$ are specified in eq. (3.10). In the glue part of the rate $A$, eq. (3.12), the $k_{1}, k_{2}$ integrals can be carried out analytically by expanding the Bose function, eq. $(3.10)$, in a power series in $\exp (-k / T)$ : 


$$
\begin{aligned}
A_{\mathrm{g}} & =\frac{4}{\pi^{4}} T \int_{4 M^{2}}^{\infty} \mathrm{d} s s^{3 / 2} \bar{\sigma}_{\mathrm{qq} \rightarrow \mathrm{s} \overline{\mathrm{s}}}(s) \sum_{n, m=1}(n m)^{-1 / 2} K_{1}\left(\frac{(n m s)^{1 / 2}}{T}\right) \\
& \simeq \frac{7}{6 \pi^{2}} \alpha_{\mathrm{s}}^{2} M T^{3} \mathrm{e}^{-2 M / T}\left(1+\frac{51}{14} \frac{T}{M}+\cdots\right) .
\end{aligned}
$$

To obtain the quark contribution the integrals must be evaluated numerically except in particular limits in which the Fermi distributions can be suitably approximated. We do not discuss this term further here, as the quark contribution is relatively unimportant, but we present the numerical results below.

Once strange quark-antiquark pairs have been created, the $s \bar{s}$-annihilation reaction will deplete the strange quark population. Under the condition of statistical independence of the creation and annihilation process, this loss term is proportional to the square of the density $\rho_{\mathrm{s}}$ of strange and antistrange quarks. With $\rho_{\mathrm{s}}^{\infty}$ being the saturation density at large times, the following differential equation then approximately describes the evolution of $\rho_{\mathrm{s}}$ as a function of time:

$$
\mathrm{d} \rho_{\mathrm{s}} / \mathrm{d} t=A\left[1-\left(\rho_{\mathrm{s}}(t) / \rho_{\mathrm{s}}^{\infty}\right)^{2}\right]
$$

We note that eq. (3.14a) may also include a term linear in $\rho_{\mathrm{s}}(t)$. For example, when the plasma density is sufficiently high, the produced strange quarks have difficulty in getting away quickly from each other. With a scattering length of the order of $\frac{1}{3} \mathrm{fm}$, in extreme cases, one has to allow for diffusion rather than ready free motion. In the limiting case of very dense medium we find the created sis pair still in a given correlation volume, and hence the annihilation term is linear in $\rho_{\mathrm{s}}$ in such instance

$$
\mathrm{d} \tilde{\rho}_{\mathrm{s}} / \mathrm{d} t \approx A\left\{1-\left[\tilde{\rho}_{\mathrm{s}}(t) / \rho_{\mathrm{s}}^{\infty}\right]\right\}
$$

The solutions of (3.14) and (3.15) are quite similar in their appearance (monotonically rising, saturating functions) and are, respectively,

$$
\begin{aligned}
& \rho_{\mathrm{s}}(t)=\rho_{\mathrm{s}}^{\infty} \tanh (t / 2 \tau) \stackrel{t \rightarrow \infty}{\longrightarrow} \rho_{\mathrm{s}}^{\infty}\left(1-2 \mathrm{e}^{-t / \tau}\right), \\
& \tilde{\rho}_{\mathrm{s}}(t)=\rho_{\mathrm{s}}^{\infty}\left[1-\mathrm{e}^{-t / \tilde{\tau}}\right]
\end{aligned}
$$

with the relaxation time constants $\tau$ and $\tilde{\tau}$ controlling the time scale of approach to the asymptotic equilibrium density:

$$
\begin{aligned}
& \tau=\rho_{\mathrm{s}}^{\infty} / 2 A, \\
& \tilde{\tau}=\rho_{\mathrm{s}}^{\infty} / A,
\end{aligned}
$$

respectively. Note that it takes twice as long to reach equilibrium if there is close $s-\bar{s}$ correlation. A factor 2 in eqs. (3.15a) and (3.16a) is missing in [RM82], but that omission just compensates for the other missing factor $1 / 2$ in front of the gluonic contribution in eq. (3.11). The results for the approach to chemical equilibrium of strange quarks due to the process $g g \rightarrow s \bar{s}$ as presented in [RM82] are therefore correct, whereas the relaxation time given for the less important process $q \bar{q} \rightarrow s \bar{s}$ 
[BZ82, RM82] was too large by a factor of two. Thus the net result, after rectifying all omissions in earlier work is that the true equilibration time is by about ten percent shorter than given in [RM82]. A contrary claim made in [Ma85] is incorrect as pointed out in [KMR86]. More importantly, the precise value of the equilibration time constant is controlled more significantly by the details of QCD interactions, the value of strange quark mass and other properties of the medium into which strange quarks are produced (see also the following subsection).

\subsection{Results for static quark-gluon plasma}

We now discuss the numerical results for the rates, time constants and the expected strangeness abundance [KMR86]. In fig. 3.2a we compare the rates for strangeness production by the processes depicted in fig. 3.1 for the parameter values $\alpha_{\mathrm{s}}=0.6, m_{\mathrm{s}}=150 \mathrm{MeV}$. We see that the gluon contribution dominates the strangeness creation rate $A$. The rate for $\mathrm{q} \overline{\mathrm{q}} \rightarrow \mathrm{s} \overline{\mathrm{s}}$ alone (shown separately - dashed lines) contributes less than 20 percent to the total rate. Using the equilibrium density of strange quarks in the plasma, (cf. section 1.3) we can therefore approximate the relaxation time $\tau$ by that determined by the gluonic production mechanism alone. The analytical result, setting $M=m_{\mathrm{s}}$ of section 2 , with $\tau$ as defined in eq. (3.16a):

$$
\tau \approx \tau_{\mathrm{g}} \simeq \frac{1.61}{\alpha_{\mathrm{s}}^{2} T} \frac{\left(m_{\mathrm{s}} / T\right) \exp \left(m_{\mathrm{s}} / T\right)}{\left(1+\frac{99}{56} T / m_{\mathrm{s}}+\cdots\right)}
$$

is falling rapidly with increasing temperature. In fig. $3.2 \mathrm{~b}$ we show the characteristic relaxation times toward chemical equilibrium, $\tau$, defined in eq. (3.16a). Again it is obvious that gluonic strangeness production is the dominant process since $\tau_{\mathrm{g}}$ is substantially smaller than $\tau_{\mathrm{q}}$ (dashed lines). If we compare the time constant $\tau$ with the estimated lifetime of the plasma state (horizontal line) we find that the strangeness abundance will be close to saturation for temperatures of $200 \mathrm{MeV}$ and above, i.e., for an
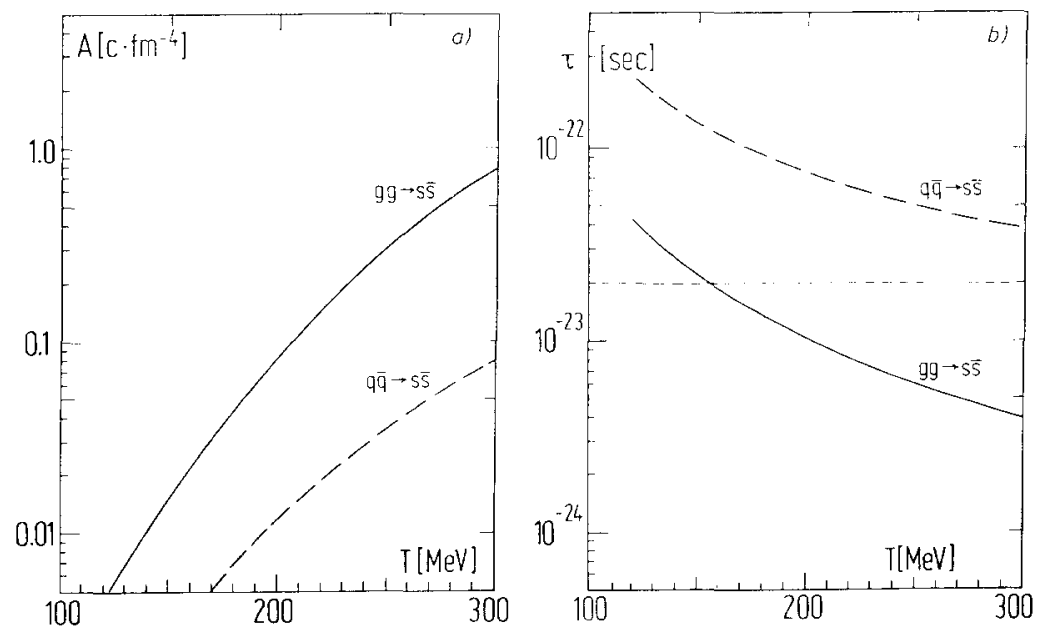

Fig. 3.2. (a) Rates $A$, (b) time constants $\tau$ as function of temperature $T$. Full lines: $q \bar{q} \rightarrow s \bar{s}$ and $g g \rightarrow s \bar{s}$; dashed lines $q \bar{q} \rightarrow s \bar{s}$. The curves are for

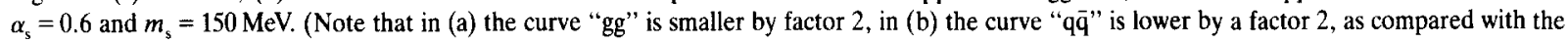
results of [RM82].) 
energy density above $1 \mathrm{GeV} / \mathrm{fm}^{3}$. We note that $\tau$ is quite sensitive to the choice of the strange quark mass parameter $m_{\mathrm{s}}$ and the coupling constant $\alpha_{\mathrm{s}}$. As we have seen in section 2 the appropriate choice of $m_{\mathrm{s}}$, as well as that of $\alpha_{\mathrm{s}}$, depends on the energy density of the plasma state.

The dependence of strangeness production on the mass of the strange quark has been studied in detail by Munehisa and Munehisa [MM84]. We show their results in figs. 3.3, corrected for the factor $1 / 2$ in the gluonic production rate. At fixed $T=200 \mathrm{MeV}$ and $\alpha_{\mathrm{s}}=0.6$ the strangeness equilibration time increases rapidly with $m_{\mathrm{s}}$ (fig. 3.3a), and the total density of produced strange quarks after a fixed lifetime $t_{\mathrm{e}}=20 \mathrm{fm} / \mathrm{c}$ of the plasma falls steeply (fig. 3.3b). This illustrates that enhanced strangeness production is also a promising signal for the chiral phase transition [Sh81a, Pi82] in high-temperature QCD if one adopts the view that quarks are endowed with a high dynamical mass which melts down to smaller values. Figure $3.3 \mathrm{~b}$ shows that a change of $200 \mathrm{MeV}$ in the effective mass $m_{\mathrm{s}}$ of the strange quark would result in almost an order of magnitude change of the strangeness abundance.

At this point we revisit again the process of creation of charmed quarks in quark-gluon plasma, which is quite different due to the fact that $m_{\mathrm{c}} / T \sim 7-10$. Due to the large value of $m_{\mathrm{c}} / T$ the density-quarks at equilibrium is very low, so that conservation of charm must be accounted for exactly and not on average, precluding the use of the grand canonical ensemble. The equilibrium density of charmed quarks is therefore [RD80] (assuming $V \leqslant 1000 \mathrm{fm}^{3}$ ):

$$
\rho_{\mathrm{c}}^{\infty} \sim g_{\mathrm{q}}^{2} V\left(m_{\mathrm{c}} T / 2 \pi\right)^{3} \exp \left(-2 m_{\mathrm{c}} / T\right)
$$

where $g_{\mathrm{q}}=6$ as in eq. (3.3) and $V$ is the quark-gluon plasma volume. Assuming for the moment that

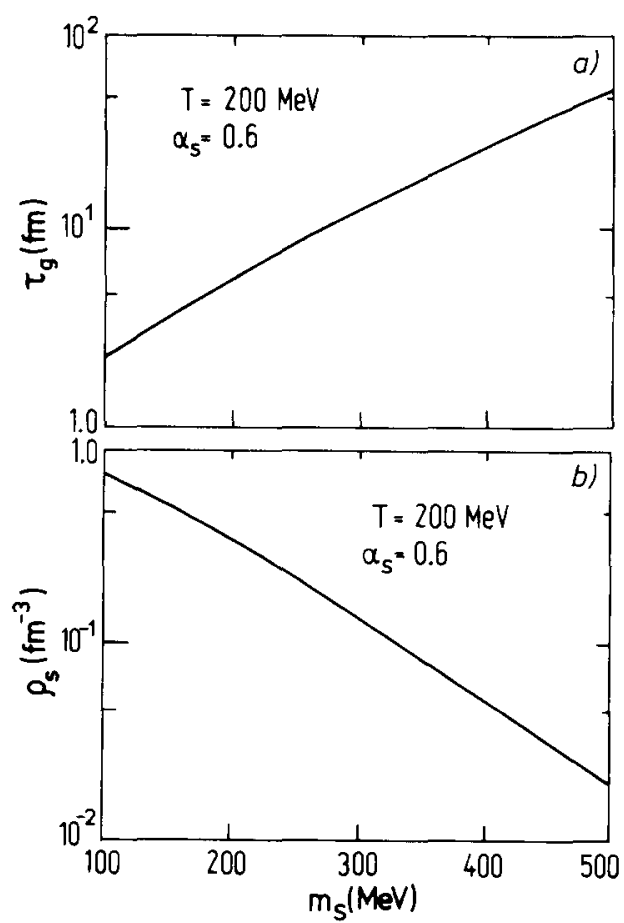

Fig. 3.3. Dependence of strange quark production on strange quark mass for $T=200 \mathrm{MeV}$ and $\alpha_{\mathrm{s}}=0.6$. (a) Equilibration time for the process $\mathrm{gg} \rightarrow \mathrm{s} \bar{s} ;$ (b) density of strange quarks after $t=20 \mathrm{fm} / c$. 
high energy gluons are available for such a far-off thermal equilibrium reaction, we have, according to eqs. (3.13) and (3.16a):

$$
\tau_{\mathrm{g}}^{(\mathrm{c})}=\rho_{\mathrm{c}}^{\infty} / 2 A_{\mathrm{g}}^{(\mathrm{c})} \approx 27 m_{\mathrm{c}}^{2} V / 14 \pi \alpha_{\mathrm{s}}^{2}=V \cdot 860 \mathrm{fm}^{-2} .
$$

Here we have used $\alpha_{\mathrm{s}}=0.2$ for the strong coupling constant at the charmed quark mass scale. Seen together with eq. (3.18) this result implies a very low charmed quark abundance at the break-up time of the quark-gluon plasma expected to live not much longer than $6 \mathrm{fm} / c$. Thus, both the absence of hard gluons mentioned already and smallness of the charm phase space contribute to a rather small abundance of charmed quarks [Ra82]. Nonetheless, detailed studies of charm production, in particular plasma gluon fusion to charmonium [CP84, CV84] have been done and the transparency of plasma to charm has been considered [Re84]. We will discuss this further in section 3.5 .

So it turns out that it is just the small window - quark mass equal to $\left(\frac{1}{2}-1\right) \times$ temperature - which makes the strangeness formation time similar to the expected lifespan of quark-gluon plasma and allows us to observe the presence of this new form of matter by the observation of consequences of large strange quark density and abundance created by gluons during the short lifetime of the plasma state. The evolution of the density of strange quarks, eq. (3.15a), relative to the baryon number content of the plasma state, is shown in fig. 3.4 for various temperatures. The saturation of the abundance is visible for $T \geq 200 \mathrm{MeV}$. The strangeness abundance shows a pronounced threshold behaviour at $T \approx 160 \mathrm{MeV}$.

In order to appreciate the anomalously large strange particle abundances emerging from quark-gluon plasma, we will consider the formation of strangeness in the case where no quark-gluon plasma has been formed and hadronic matter consists of individual hadrons in detail in section 5. In particular, the approach to chemical equilibrium abundances in the hadronic gas is of importance and it will turn out that the absence of the equilibrium abundances in the hadronic gas helps distinguish between the two different states of hadronic matter at the level of singly strange hadrons. Quark-gluon plasma acts as a source of strangeness, facilitating rapid saturation of the (hadronic gas) phase space. Absence of gluonic degrees of freedom assures that far too little strangeness is produced in individual hadronic (gas) collisions.

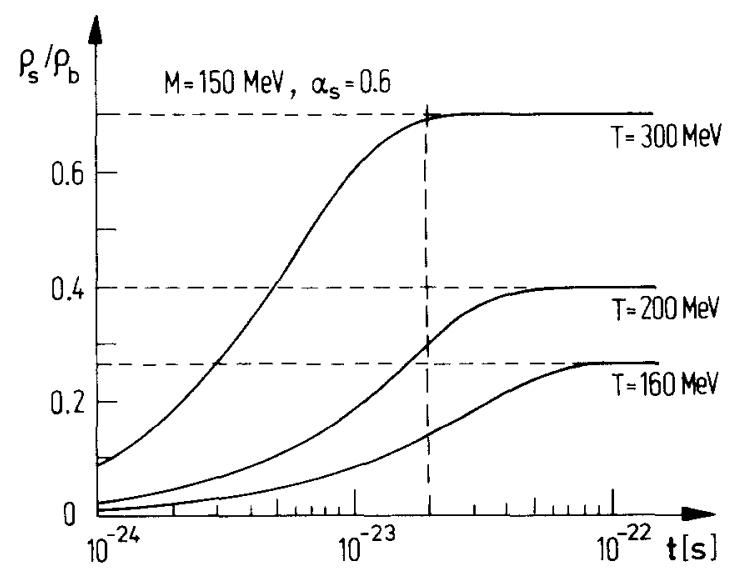

Fig. 3.4. Time evolution of the relative strange quark to baryon number abundance in the plasma for various temperatures $T$ ( $m_{\mathrm{s}}=150 \mathrm{MeV}$, $\left.\alpha_{\mathrm{s}}=0.6\right)$. 


\subsection{Influence of volume expansion}

When the volume occupied by the quark-gluon plasma changes in time (expands), we have to supplement eq. (3.14a) with the volume dilution term (see section 7 for further extensive discussion)

$$
\frac{\mathrm{d} \rho_{\mathrm{s}}}{\mathrm{d} t}=-\rho_{\mathrm{s}} \frac{1}{V} \frac{\mathrm{d} V}{\mathrm{~d} t}+A\left(1-\left(\rho_{\mathrm{s}} / \rho_{\mathrm{s}}^{\infty}\right)^{2}\right)
$$

where now $A$ and $\rho_{\mathrm{s}}^{\infty}$ are time dependent quantities in consequence of temperature being a time dependent function. Both $A_{\mathrm{g}} \approx A$ and $\rho_{\mathrm{s}}^{\infty}$ are not dependent on the chemical potential of light quarks. As described in section 1, we assume the simple functional relations, eq. (1.18) to parameterize the time dependence of $T$ and $V$. In fig. 3.5a the resulting evolution of strange particle density, calculated
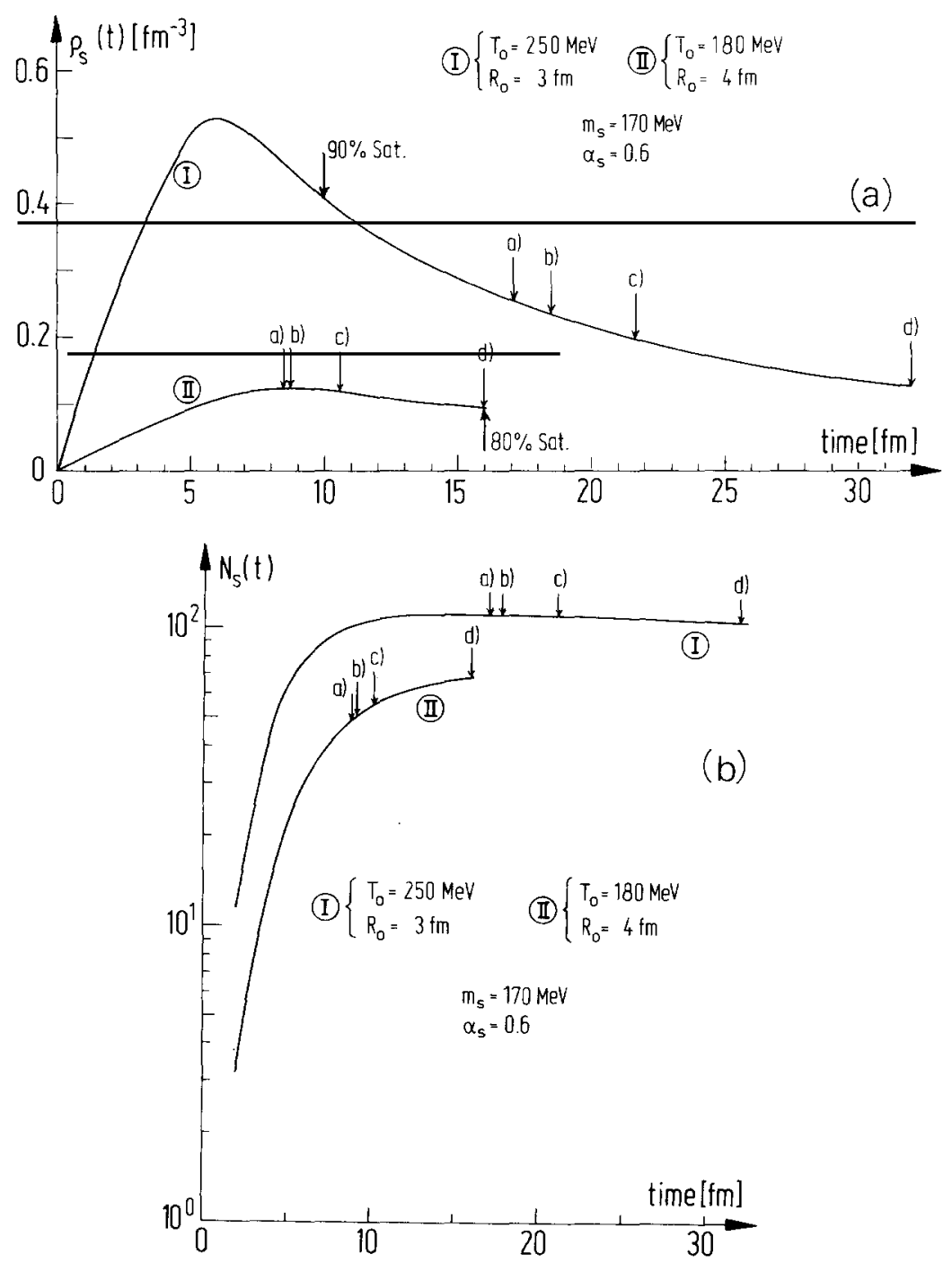

Fig. 3.5. (a) Evolution of strangeness density for two typical evaluation scenarios of the plasma. See text for explanations. (b) Total strangeness evolution in plasma $\left(\rho_{\mathrm{s}}(t)\right.$ multiplied with $\left.V(t)\right)$. 
assuming $m_{\mathrm{s}}=170 \mathrm{MeV}, \alpha_{\mathrm{s}}=0.6$ is shown [KMR86]. Case I corresponds to a rather hot blob of plasma of size $3 \mathrm{fm}$ and initial $T=250 \mathrm{MeV}$, while case II corresponds to the initial plasma temperature being more modest, $T_{0}=180 \mathrm{MeV}$, while the radius being $4 \mathrm{fm}$. $V \sim t$ has been assumed here. Since the product $T \cdot R$ is nearly the same, the energy content is almost equal in both examples. They hence represent two different conceivable scenarios of a collision of heavy nuclei. The arrow marked $90 \%$ shows the time at which the phase space saturation reaches that level. The fall of the strangeness density is a consequence of the volume expansion, rather than strangeness annihilation. As shown in fig. 3.5b, the total strangeness is quite rapidly built up in the collision, and we can expect 80-120
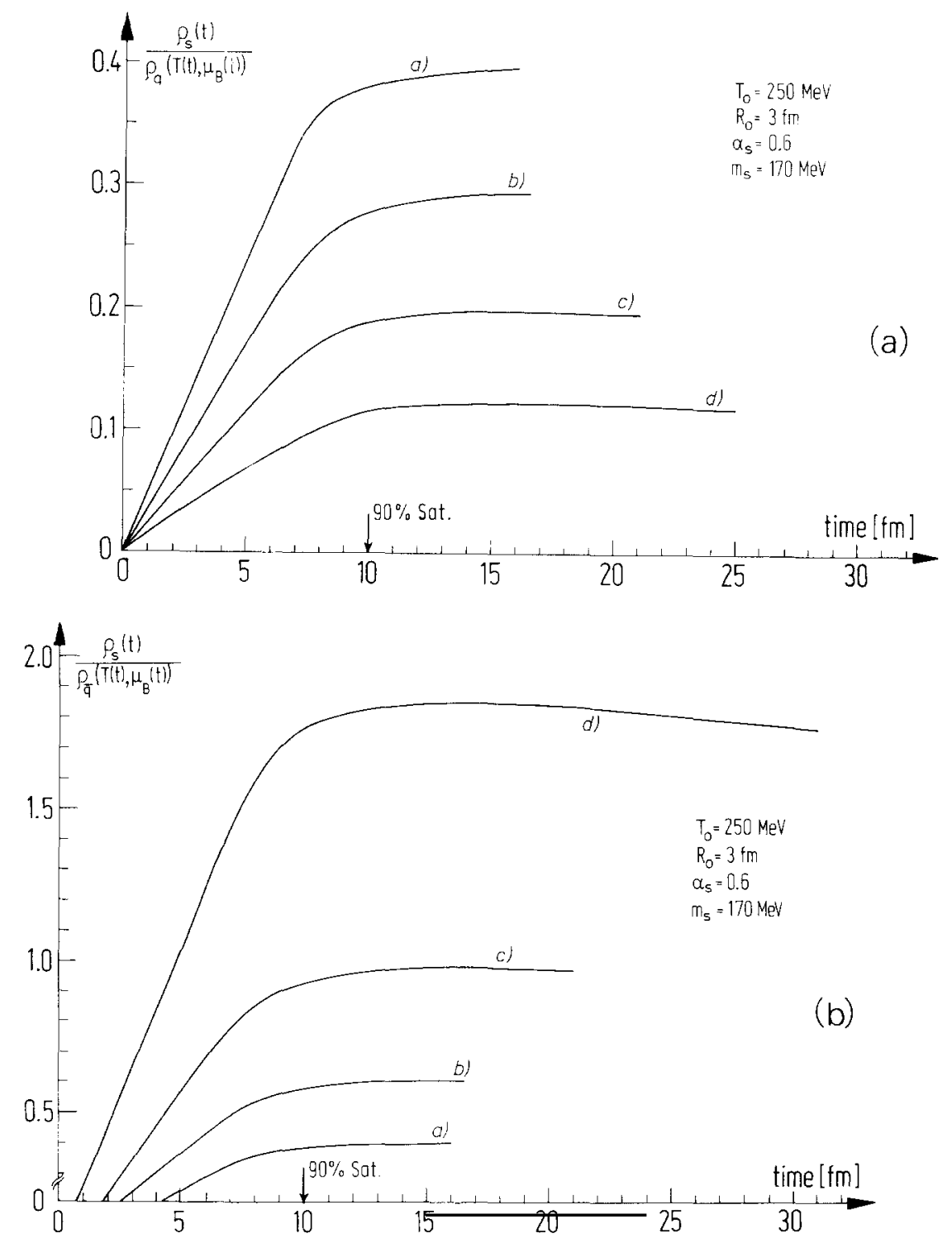

Fig. 3.6. Relative abundance of strangeness: (a) $\rho_{\mathrm{s}} / \rho_{\mathrm{q}}$, (b) $\rho_{\mathrm{s}} / \rho_{\overline{\mathrm{q}}}$. 
strange quark pairs to be produced. There is virtually no net strangeness annihilation $s \bar{s} \rightarrow g g$ as the plasma expands. This is because in the expansion process the temperature and strangeness density both drop and the combined effect is sufficient to rapidly decouple strangeness from the hadronic reequilibration reactions. Points (a), (b), (c) and (d) indicate when, along the assumed critical curve (see fig. 1.3), the critical temperature is reached at which the chemical potential assumes the values 0,200 , $400,600 \mathrm{MeV}$, respectively (see table 1.1 ), corresponding to $T_{\mathrm{cr}}=160,156,148,130 \mathrm{MeV}$, respectively. The phase transition to the hadronic gas occurs at these points, depending on baryon content of the plasma. As we see from fig. 3.5a, the transition density is expected in the narrow interval $0.15 / \mathrm{fm}^{3}<$ $\rho_{\mathrm{s}}<0.3 / \mathrm{fm}^{3}$. This value indicates that clustering of two strange quarks in one hadronic volume will be frequent, and further that the total abundance of strangeness is a measure of the plasma volume at the phase transition [KMR86].

Under the assumption of ideal gas relations between the baryochemical potential $\mu_{\mathrm{b}}$ and the $\mathrm{q}=\mathrm{u}+\mathrm{d}$ and $\overline{\mathrm{q}}$ light flavour densities, we compute the time evolution of relative strangeness abundance evaluation $\rho_{\mathrm{s}}(t) / \rho_{\mathrm{q}}(t)$ and $\rho_{\overline{\mathrm{s}}}(t) / \rho_{\overline{\mathrm{q}}}(t)$ for case I, shown in fig. 3.6. Curves marked (a)-(d) correspond to values of $\mu_{\mathrm{b}} / T$ as fixed in table 1.1 and discussed above. Naturally, with increasing $\mu_{\mathrm{b}}$ the relative strangeness abundance $\rho_{\mathrm{s}} / \rho_{\mathrm{q}}$ diminishes, but is substantial even at large $\mu_{\mathrm{b}}$. As baryon number increases, $\overline{\mathrm{q}}$ abundance decreases and the opposite trend results in the $\rho_{\overline{\mathrm{s}}} / \rho_{\overline{\mathrm{q}}}$ abundance. These two characteristic points have been emphasized previously [RH80, Ra81, Ra82, Ra84, RD83] and are essential prerequisites in order that the proposed anomalous abundances of strange antibaryons develop in nuclear collisions. We record, in particular, that at $\mu_{\mathrm{b}} / T \sim 2.7$ (curve (c)), we expect equal numbers of $\overline{\mathrm{s}}$ and $\overline{\mathrm{u}}+\overline{\mathrm{d}}$ quarks, while for each 2.5 nonstrange quarks we find one s or $\overline{\mathrm{s}}$ quark in the plasma.

One may argue that the selected scenario in respect of volume or temperature of the plasma has been overly simplified and optimistic with regard to the strangeness phase space saturation in plasma. As the main developments of our work depend nearly exclusively on the ability of the plasma state to reach the equilibrium strangeness density we now will show that this is quite generally the case. The evolution of the plasma depends on two initial quantities: $R_{0}$ and $T_{0} ; R_{0}$ controls the characteristic time of the plasma, see eq. (1.1c), but the lifetime is prolonged by having high initial temperature $T_{0}$. Further, high temperature greatly facilitates the formation of strangeness, cf. eq. (3.17). All these

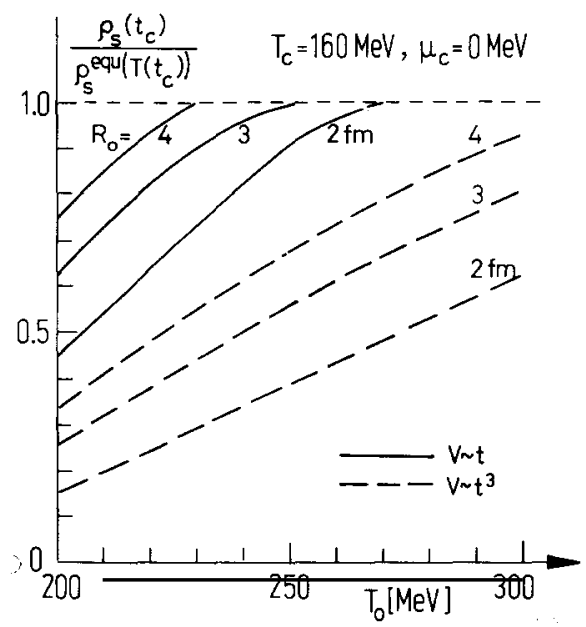

Fig. 3.7. Degree of strangeness abundance equilibration as a function of initial temperature $T_{0}$. The radius of the plasma is a parameter: $R_{0}=2,3$, $4 \mathrm{fm}$. 
influences can be systematically investigated by considering the degree of strangeness equilibration reached in the plasma as a function of $T_{0}$ with $R_{0}$ being a parameter. In fig. 3.7 we see that to within factor 2 we always achieve saturation of strangeness phase space, of course more easily for the long-lived large plasma or a hot initial environment. Here $t_{\mathrm{c}}$ is the time at which the plasma state cools to the phase transition temperature and commences to coexist with the hadronic gas phase (see section 1 and table 1.1).

\subsection{Influence of Pauli blocking of final states}

Matsui, Svetitsky and McLerran [Ma85] have recently studied the influence of final state Pauli blocking on the rate constants for the strangeness production processes $g g \rightarrow s \bar{s}$ and $q \bar{q} \rightarrow s \bar{s}$. As more and more strange quarks and antiquarks are produced they occupy, in part, the available phase space and may impede production through the influence of the Pauli exclusion principle. In order to account for this effect, it is necessary to make additional assumptions beyond those introduced in section 3.1, because the phase space distribution of already produced strange quarks must be identified. Matsui et al. assumed that the strange quarks produced are rapidly thermalized, so that their momentum distribution can be represented by the form

$$
f_{\mathrm{s}}(k)=f_{\overline{\mathrm{s}}}(k)=\left(\lambda_{\mathrm{s}}^{-1} \mathrm{e}^{\beta \cdot k}+1\right)^{-1}
$$

Here $\lambda_{\mathrm{s}} \equiv \exp \left(\mu_{\mathrm{s}} / T\right)$ is the fugacity of strange quarks which describes the amount of saturation of the statistical phase space density. Initially, for $\rho_{\mathrm{s}}=0$, we have $\lambda_{\mathrm{s}}=0\left(\mu_{\mathrm{s}}=-\infty\right)$; chemical equilibrium is characterized by $\lambda_{\mathrm{s}}=1\left(\mu_{\mathrm{s}}=0\right)$. Since strange quarks are always produced in pairs, $\rho_{\mathrm{s}}$ and $\rho_{\overline{\mathrm{s}}}$ are equal, which implies $\lambda_{\mathrm{s}}=\lambda_{\overline{\mathrm{s}}}$. As the chemical equilibrium requires $\lambda_{\overline{\mathrm{s}}}=\lambda_{\mathrm{s}}^{-1}$, the equilibrium abundance must satisfy $\lambda_{\mathrm{s}}=\lambda_{\overline{\mathrm{s}}}=1$ as stated. While assumption of eq. (3.21) seems, at first sight, perfectly reasonable, it follows upon far-reaching assumptions, e.g. that the space correlations between the quark and the antiquark of a newly produced strange quark pair are rapidly destroyed.

Once $f_{\mathrm{s}}(k)$ and $f_{\overline{\mathrm{s}}}(k)$ are specified (cf. eq. (3.21)), it is easy to express the production rate $A_{\text {gain }}$ and the annihilation rate $A_{\text {loss }}$ of strange quarks in terms of the invariant matrix elements (3.1) and the phase space densities, taking Pauli blocking and effects of Bose statistics into account:

$$
\begin{aligned}
A_{\text {gain }}= & \int \frac{\mathrm{d}^{3} k_{1}}{(2 \pi)^{3} 2\left|k_{1}\right|} \int \frac{\frac{\mathrm{d}^{3} k_{2}}{(2 \pi)^{3} 2\left|k_{2}\right|} \int \frac{\mathrm{d}^{3} p_{1}}{(2 \pi)^{3} 2\left|p_{1}\right|} \int \frac{\mathrm{d}^{3} p_{2}}{(2 \pi)^{3} 2\left|p_{2}\right|}(2 \pi)^{4} \delta\left(p_{1}+p_{2}-k_{1}-k_{2}\right)}{}+\times\left[\frac{1}{2} f_{\mathrm{g}}\left(k_{1}\right) f_{\mathrm{g}}\left(k_{2}\right) \sum\left|\mathcal{M}_{\mathrm{a}}+\mathcal{M}_{\mathrm{b}}+\mathcal{M}_{\mathrm{c}}\right|^{2}+f_{\mathrm{q}}\left(k_{1}\right) f_{\mathrm{q}}\left(k_{2}\right)\left|\mathcal{M}_{\mathrm{d}}\right|^{2}\right]\left[1-f_{\mathrm{s}}\left(p_{1}\right)\right]\left[1-f_{\overline{\mathrm{s}}}\left(\rho_{2}\right)\right] \\
A_{\text {loss }}= & \int \frac{\mathrm{d}^{3} k_{1}}{(2 \pi)^{3} 2\left|k_{1}\right|} \int \frac{\mathrm{d}^{3} k_{2}}{(2 \pi)^{3} 2\left|k_{2}\right|} \int \frac{\mathrm{d}^{3} p_{1}}{(2 \pi)^{3} 2\left|p_{1}\right|} \int \frac{\mathrm{d}^{3} p_{2}}{(2 \pi)^{3} 2\left|p_{2}\right|}(2 \pi)^{4} \delta\left(\rho_{1}+p_{2}-k_{1}-k_{2}\right) \\
& \times\left[\frac{1}{2}\left(1+f_{\mathrm{g}}\left(p_{1}\right)\right)\left(1+f_{\mathrm{g}}\left(p_{2}\right)\right) \sum\left|\mathcal{M}_{\mathrm{a}}+\mathcal{M}_{\mathrm{b}}+\mathcal{M}_{\mathrm{c}}\right|^{2}\right. \\
& \left.+\left(1-f_{\mathrm{q}}\left(p_{1}\right)\right)\left(1-f_{\overline{\mathrm{q}}}\left(p_{2}\right)\right) \sum\left|\mathcal{M}_{\mathrm{d}}\right|^{2}\right] f_{\mathrm{s}}\left(k_{1}\right) f_{\overline{\mathrm{s}}}\left(k_{2}\right)
\end{aligned}
$$


The factor $1 / 2$ in front of the glue contributions again account for the double-counting of gluon pairs. In eqs. (3.22) and (3.23) the law of detailed balance is incorporated, i.e. the squared invariant matrix elements, summed over degeneracy of initial and final states, are equal for the forward and reverse reaction. Further, since

$$
\sigma=\frac{1}{4 k_{1} \cdot k_{2}} \int \frac{\mathrm{d}^{3} p_{1}}{(2 \pi)^{3} 2\left|p_{1}\right|} \int \frac{\mathrm{d}^{3} p_{2}}{(2 \pi)^{3} 2\left|p_{2}\right|}(2 \pi)^{4} \delta\left(p_{1}+p_{2}-k_{1}-k_{2}\right)|\mathcal{M}|^{2}=\frac{1}{16 \pi s^{2}} \int_{t_{-}}^{t_{+}} \mathrm{d} t|\mathcal{M}|^{2},
$$

we can establish the relation with our previous eq. (3.11), provided that the statistical factors $\left(1-f_{\mathrm{s}}\right)$, $\left(1-f_{\bar{s}}\right)$ are replaced by one [KMR86]. This amounts to the neglect of Pauli blocking in the final state. Observing the elementary properties of the thermal distributions, viz.

$$
\begin{aligned}
& 1+f_{\mathrm{g}}(k)=\exp (\beta \cdot k) f_{\mathrm{g}}(k), \\
& 1-f_{\mathrm{q}}(k)=\exp \left(\beta \cdot k-\mu_{\mathrm{q}} / T\right) f_{\mathrm{q}}(k), \\
& 1-f_{\overline{\mathrm{q}}}(k)=\exp \left(\beta \cdot k-\mu_{\mathrm{q}} / T\right) f_{\overline{\mathrm{q}}}(k), \\
& 1-f_{\mathrm{s}}(k)=1-f_{\overline{\mathrm{s}}}(k)=\lambda_{\mathrm{s}}^{-1} \exp (\beta \cdot k) f_{\mathrm{s}}(k),
\end{aligned}
$$

it is possible to rewrite the effective reaction rate as

$$
\begin{aligned}
A_{\text {eff }}\left(\lambda_{\mathrm{s}}\right) & \equiv A_{\text {gain }}\left(\lambda_{\mathrm{s}}\right)-A_{\text {loss }}\left(\lambda_{\mathrm{s}}\right) \\
& =\left(1-\lambda_{\mathrm{s}}^{2}\right) A_{\text {gain }}\left(\lambda_{\mathrm{s}}\right)=\left(\lambda_{\mathrm{s}}^{-2}-1\right) A_{\text {loss }}\left(\lambda_{\mathrm{s}}\right) .
\end{aligned}
$$

Upon approach to chemical equilibrium, i.e. $\lambda_{\mathrm{s}} \rightarrow 1$, the overall reaction rate $A_{\text {eff }}$ obviously tends to zero as

$$
A_{\mathrm{eff}}\left(\lambda_{\mathrm{s}}\right) \underset{\lambda_{\mathrm{s}} \rightarrow 1}{\longrightarrow}\left(1-\lambda_{\mathrm{s}}\right) 2 A_{\mathrm{gain}}\left(\lambda_{\mathrm{s}}=1\right) .
$$

In order to determine the relaxation time near the equilibrium distribution which may be expected to be influenced by Pauli blocking, one now has to study the dependence of the strange quark density $\rho_{\mathrm{s}}$ on the fugacity $\lambda_{\mathrm{s}}$ near $\lambda_{\mathrm{s}}=1$ :

$$
\begin{aligned}
\frac{\partial}{\partial \lambda_{\mathrm{s}}} \rho_{\mathrm{s}} & =\frac{\partial}{\partial \lambda_{\mathrm{s}}} \int \frac{\mathrm{d}^{3} k}{(2 \pi)^{3}}\left(\lambda_{\mathrm{s}}^{-1} \mathrm{e}^{\beta \cdot k}+1\right)^{-1} \\
& =\lambda_{\mathrm{s}}^{-1}\left[\rho_{\mathrm{s}}-\int \frac{\mathrm{d}^{3} k}{(2 \pi)^{3}}\left(\lambda_{\mathrm{s}}^{-1} \mathrm{e}^{\beta \cdot k}+1\right)^{-2}\right] \\
& \underset{\lambda_{\mathrm{s}} \rightarrow 1}{\longrightarrow} \rho_{\mathrm{s}}^{\infty}-\int \frac{\mathrm{d}^{3} k}{(2 \pi)^{3}}\left(\mathrm{e}^{\beta \cdot k}+1\right)^{-2} \equiv \rho_{\mathrm{s}}^{\infty^{\prime}}
\end{aligned}
$$


The evolution equation

$$
\mathrm{d} \rho_{\mathrm{s}} / \mathrm{d} t=A_{\text {eff }}\left(\lambda_{\mathrm{s}}\right)
$$

then takes the asymptotic form

$$
\rho_{\mathrm{s}}^{\infty^{\prime}} \mathrm{d} \lambda_{\mathrm{s}} / \mathrm{d} t \approx\left(1-\lambda_{\mathrm{s}}\right) 2 A_{\text {gain }}^{\prime} \quad\left(\lambda_{\mathrm{s}} \rightarrow 1\right)
$$

with $A_{\text {gain }}^{\prime}=A_{\text {gain }}\left(\lambda_{\mathrm{s}}=1\right)$. This saturation equation has the same asymptotic solution as eq. (3.14a), with the equilibration time constant

$$
\tau^{\prime}=\rho_{\mathrm{s}}^{\infty^{\prime}} / 2 A_{\mathrm{gain}}^{\prime}
$$

The relaxation time (3.31) has been evaluated numerically by Matsui et al. [Ma85]. In addition to the effects of Fermi and Bose statistics for light quarks and gluons, which are already contained in eq. (3.16a), it aso contains the influence of Pauli blocking. This effect will be of relevance only for high equilibrium density of strange quarks, i.e. at temperatures $T$ considerably higher than the strange quark mass $m_{\mathrm{s}}$, as shown in fig. 3.8. The solid line represents the strangeness equilibration time as defined in eq. (3.31), while the dashed line reproduces the previous result (3.16a), which neglects the Pauli blocking in the strange quark phase space [KMR86]. The small difference is seen to grow gradually with temperature, not exceeding 20 percent even up to $T=400 \mathrm{MeV}$, which corresponds to an energy density of about $20 \mathrm{GeV} / \mathrm{fm}^{3}$.

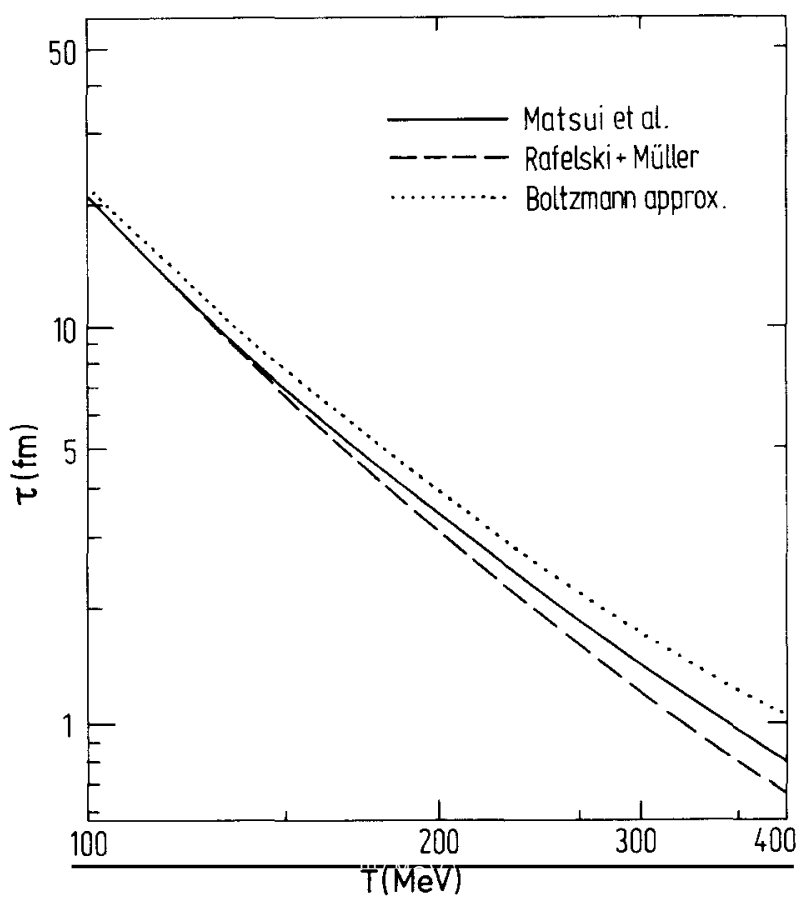

Fig. 3.8. Influence of Pauli blocking on the strangeness equilibration rate. Solid line: with Pauli blocking; dashed line: without Pauli blocking [Ma85]; dotted line: Boltzmann statistics. 
The dotted curve shows the result obtained if all phase space distribution functions in eq. (3.22) are replaced by the classical (Boltzmann) distribution. This substitution leads to a somewhat lower rate of strangeness production, mainly through partial suppression of gluonic degrees of freedom in absence of Bose statistics. It is obvious here that the classical momentum distributions are sufficient for a quantitative estimate of the strangeness equilibration time in the quark-gluon plasma, given the much larger uncertainties pertaining to the precise values of the relevant parameters $m_{\mathrm{s}}$ and $\alpha_{\mathrm{s}}$.

\subsection{Pre-equilibrium production of $J / \psi$ particles}

We now briefly return to discuss the production of charmed quark-antiquark pairs. While charmed quarks are probably not a very useful signature of the quark-gluon plasma, because the energies available in the thermally equilibrated phase space distributions are far too low, charmed quarks may be readily produced in the pre-equilibrium phase of the collision, and may therefore be an important background source of high-energy leptons. In fact, in hadronic collisions at high energy the $J / \psi$ is the main source of lepton pairs with invariant mass above $3 \mathrm{GeV}$ and not the Drell-Yan process [CP84]. This is so because of the $15 \%$ branching ratio for the decay of $J / \psi$ into $e^{+} e^{-}$and $\mu^{+} \mu^{-}$.

The diagrams of fig. 3.1 are not appropriate for description of charmonium production because the quark pair in the final state normally does not carry the appropriate quantum numbers (colour singlet, spin triplet). Cleymans and Philippe [CP84] have, therefore, considered the higher order diagrams of fig. 3.9, in which the quantum number balance is achieved by emission of a (soft) gluon in addition to the $\mathrm{J} / \psi$. Recalling the discussion of subsections 3.1 and 3.4 , the rate of $\mathrm{J} / \psi$-production by two gluons is:

$$
\begin{aligned}
A_{\mathrm{J} / \psi}= & \frac{1}{2} \int \frac{\mathrm{d}^{3} k_{1}}{(2 \pi)^{3} 2\left|k_{1}\right|} \int \frac{\mathrm{d}^{3} k_{2}}{(2 \pi)^{3} 2\left|k_{2}\right|} \int \frac{\mathrm{d}^{3} p_{\mathrm{J}}}{(2 \pi)^{3} 2 E_{\mathrm{J}}} \int \frac{\mathrm{d}^{3} p^{\prime}}{(2 \pi)^{3} 2\left|p^{\prime}\right|}(2 \pi)^{4} \\
& \times \delta\left(k_{1}+k_{2}-p_{\mathrm{J}}-p^{\prime}\right) g_{\mathrm{G}}^{2} f_{\mathrm{g}}\left(k_{1}\right) f_{\mathrm{g}}\left(k_{2}\right) \sum\left|\bar{M}_{\mathrm{gg} \rightarrow \mathrm{Jg}}\right|^{2}\left(1+f_{\mathrm{g}}\left(p^{\prime}\right)\right) \\
= & \frac{1}{2} \int \frac{\mathrm{d}^{3} p_{\mathrm{J}}}{(2 \pi)^{2} 2 E_{\mathrm{J}}} \int \frac{\mathrm{d}^{3} k_{1}}{(2 \pi)^{3} 2\left|k_{1}\right|} \int \frac{\mathrm{d}^{3} k_{2}}{(2 \pi)^{3} 2\left|k_{2}\right|} \delta\left(\left(k_{1}+k_{2}-p_{\mathrm{J}}\right)^{2}\right) g_{\mathrm{G}}^{2} f_{\mathrm{g}}\left(k_{1}\right) f_{\mathrm{g}}\left(k_{2}\right) \\
& \times \sum\left|\bar{M}_{\mathrm{gg} \rightarrow \mathrm{Jg}}\right|^{2} .
\end{aligned}
$$

Here we have neglected Pauli blocking effects for charmed quarks, and in the second step also induced emission of the final state gluon. We also made use of the masslessness of the gluon in the delta function. The overall factor $1 / 2$ introduced to avoid double counting of gluon pairs is also missing in

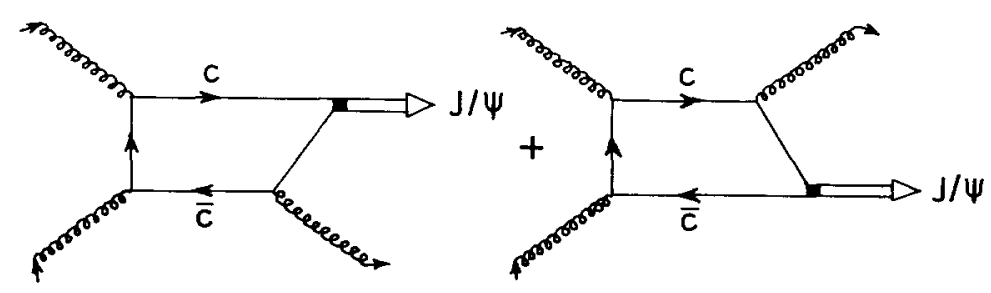

Fig. 3.9. QCD diagrams for charmonium $\mathrm{J} / \psi$ production by gluons. 
[CP84]. The invariant matrix element, summed over final and averaged over initial quantum numbers is:

$$
\sum\left|\bar{M}_{\mathrm{gg} \rightarrow \mathrm{Jg}}\right|^{2}=5 \pi^{2} \alpha_{\mathrm{s}}^{3} M_{\mathrm{J}}^{3} \frac{\Gamma\left(\mathrm{J} / \psi \rightarrow \mu^{+} \mu^{-}\right)}{\alpha^{2}} \frac{s^{2}\left(s-M_{\mathrm{J}}^{2}\right)^{2}+t^{2}\left(t-M_{\mathrm{J}}^{2}\right)^{2}+u^{2}\left(u-M_{\mathrm{J}}^{2}\right)^{2}}{\left(s-M_{\mathrm{J}}^{2}\right)^{2}\left(t-M_{\mathrm{J}}^{2}\right)^{2}\left(u-M_{\mathrm{J}}^{2}\right)^{2}}
$$

where $M_{\mathrm{J}}$ is the mass of the $\mathrm{J} / \psi$ resonance. The leptonic decay width of the $\mathrm{J} / \psi$ is used to express the quark-antiquark wavefunction at the origin. Of course, the strong coupling constant $\alpha_{\mathrm{s}}$ must now be taken at $Q^{2}=M_{\mathrm{J}}^{2}$

The rate (3.22) and the momentum spectrum of $\mathrm{J} / \psi$ particles has been calculated by Cleymans et al. [CP84, CV84] and by Reusch [Re84] on the basis of two different assumptions for the gluon distribution $f_{\mathrm{g}}(k)$ : (i) for the thermal distribution (3.10a) in the quark-gluon plasma ('thermal production'), and (ii) for the unperturbed distribution of gluons in the incoming nucleons ('direct production'). The latter assumption corresponds to a picture of the nucleus-nucleus collision as an ensemble of $A_{1} \cdot A_{2}$ independent nucleon-nucleon collisions, where $A_{i}$ are the atomic numbers of the colliding nuclei. Note that in this latter case the factor $1 / 2$ in eq. (3.32) must be dropped as the two gluons originate from different sources, and double-counting cannot occur. In fig. 3.10 the results (full line pre-equilibrium, dashed line equilibrium production rate) are compared showing that direct production of $\mathrm{J} / \psi$ particles can be expected to be dominating over the thermal production in most regions of phase space. This makes charm production rather unattractive as possible signature for the quark-gluon plasma, but

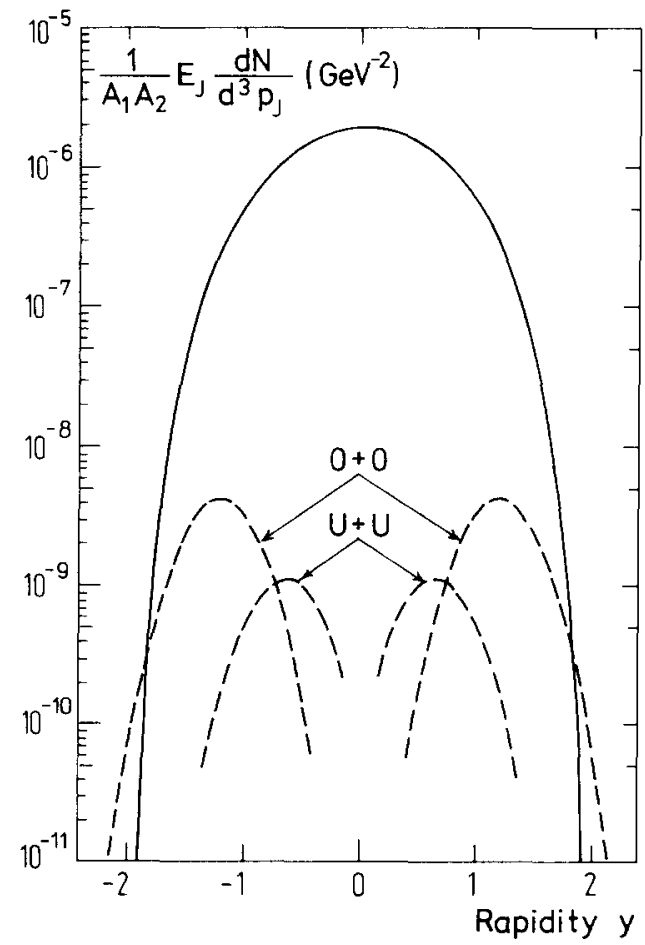

Fig. 3.10. Charmonium production rate for $\mathbf{J} / \psi$ production in nucleus-nucleus collisions, divided by product of atomic numbers. Solid line: direct production (individual nucleon-nucleon collisions) at c.m. energy $22.5 \mathrm{GeV} /$ nucleon. Dashed lines: thermal production at $T=250 \mathrm{MeV}$. The transverse momentum was taken as $250 \mathrm{MeV} / \mathrm{c}$ (after [CP84]). 
indicates that direct $\mathrm{J} / \psi$ production may constitute a serious background for lepton pairs of large invariant mass when such are used to diagnose quark-gluon plasma.

\section{Final remarks}

We thus have learned that only the strange quark abundance nearly saturates in a sufficiently excited quark-gluon plasma with $T \geqslant 200 \mathrm{MeV}, \varepsilon \geqslant 1 \mathrm{GeV} / \mathrm{fm}^{3}$ owing to the high gluon density and $m_{\mathrm{s}} \leqslant T$. This allows strangeness to be an important observable indicating in turn the abundant presence of gluons in the reaction. The high particle density of strange quarks in plasma assures that when the quark matter hadronizes some of the numerous $\mathrm{s}$ and $\overline{\mathrm{s}}$ quarks may form strangeness clusters such as $\Xi, \Omega$, and particularly important, their antiparticles, and also exotic strange objects, instead of being bound in kaons only. Consequently, we will emphasize below in our investigation, our expectations about these multistrange hadrons.

If the plasma state froze out directly into a low density hadron gas, we would expect the formation of an extraordinarily high strange quark density, with an anonymously large abundance of strange antibaryons as compared with antinucleons. In a dynamical collision process, initial high hyperon abundance will be redistributed by the processes of expansion and mixing (see section 7). More pronounced effects can be foreseen here for multistrange antihyperons due to high absolute strangeness abundance. The remainder of this report will be devoted to the development of the necessary tools and a calculation of the expected particle abundances.

\section{Conversion from the quark-gluon phase to the hadronic phase (Hadronization of the quark-gluon plasma)}

\subsection{Hadronization scenarios}

Very little is known about the process of hadron formation from a cooling quark-gluon plasma. It is not even clear whether hadronization in the context of a nuclear collision proceeds in bulk, by formation of bubbles of hadronic matter coexisting with the quark-gluon phase or by evaporation of hadrons from the surface. The latter process, in particular meson emission from the surface of the plasma, has been considered by several authors [DR83, BGM83, Sch84, ME85]. Detailed calculations have indicated that it probably is a slower process than has been initially estimated. A crude estimate of the plasma lifetime subject to pion radiation can be obtained [Mü85] by dividing the total energy contained in the plasma volume by the energy flux per unit time carried away by a pion blackbody radiation $\left(m_{\pi} \approx 0\right)$

$$
c \tau \sim \frac{c E_{-E_{\mathrm{QG}}}}{\mathrm{d} E_{\pi} / \mathrm{d} t}=\frac{\left(\pi^{2} / 30\right) g_{\mathrm{QG}} T^{4}-\pi R^{2} L}{\left(\pi^{2} / 120\right) g_{\pi} T^{4} 2 \pi R L}=\frac{2 g_{\mathrm{QG}}}{N_{\pi}} R \sim 25 R
$$

where $t_{\mathrm{c}}=37, g_{\pi}=3$ and we have assumed a cylindrical geometry with length $L$ and diameter $R$. For a transverse extension $R \sim 3 \mathrm{fm}$, which may be reasonable for collisions of heavy nuclei, we find $t_{\mathrm{h}} \sim 75 \mathrm{fm} / c$.

Other authors have assumed slow hadronization in bulk, where a mixed phase of quark-gluon plasma and hadronic matter exists for an extended period of time [KM85, FKR85]. Kapusta and Mekjian, 
taking only longitudinal expansion of the fireball into account, find that the mixed phase exists for a period

$$
t_{\mathrm{h}} \sim\left(g_{\mathrm{QG}} / g_{\pi}\right) t_{\mathrm{c}} \sim 12 t_{\mathrm{c}}
$$

where $t_{\mathrm{c}}$ is the time it takes for the quark-gluon plasma to cool to the critical temperature for hadronization. For an initial temperature $T_{0} \sim 300 \mathrm{MeV}$ they find $t_{\mathrm{h}} \sim 75 \mathrm{fm} / c$. Hence we conclude that surface radiation and bulk transition to a mixed phase are competing processes with roughly equal timescales. In section 7 we examine the consequences of a simple dynamical picture and find similar magnitudes for $t_{\mathrm{h}}$.

Another possibility is an expansion driven supercooling of the quark-gluon plasma possibly followed by sudden and explosive transition to a superheated hadronic phase. In recent calculations [KM85] such supercooling occurs for a rather long period of time $(5-50 \mathrm{fm} / c$ depending on the initial temperature). We note that this is a very much longer time than the characteristic timescale for equilibration processes in the quark-gluon plasma $\left(\tau_{\mathrm{eq}} \leqslant 0.5 \mathrm{fm} / \mathrm{c}\right)$. Since in plasma quarks and antiquarks assemble easily into hadronlike clusters [CCR84] due to their interaction, it is our opinion that hadronization itself will then not require a subsequent complete long-range reordering of the phase structure. In our view, this scenario is therefore unlikely to coexist with particle emission processes and other faster disintegration paths of the plasma.

\subsection{Fragmentation of quarks and gluons}

In the following we shall explore the consequences of a schematic fragmentation-recombination model for hadronization that is an extended and revised version of the combinatoric break-up model of Biro and Zimanyi [BZ83]. The model may be expected to provide a reasonable description of the hadronization process when it occurs in bulk without extended supercooling and subsequent reheating.

The break-up model in its initial form was based on the combinatoric distribution of quarks and antiquarks into mesons and baryons, taking into account all possible configurational degeneracies. The main reason why this model cannot provide an accurate description of the hadronization process is that the number of existing particles is reduced by about a factor of four if only recombination is assumed: each quark-antiquark pair reduces to a single meson, and the hadronization of gluons - which are almost as abundant as quarks and antiquarks combined - has been neglected. Although the reduction in the number of particles is a well-known phenomenon in chemical recombination processes, it contradicts basic laws of statistical physics when applied to a gas of relativistic particles. For a gas of massless fermions or bosons at zero chemical potential the entropy density is strictly proportional to the number density of particles, in particular

$$
\begin{aligned}
& (s / n)_{\text {bosons }}=4 \zeta(4) / \zeta(3) \sim 3.6 \\
& (s / n)_{\text {fermions }}=4 \times 7 \zeta(4) / 6 \zeta(3) \sim 4.2 .
\end{aligned}
$$

In the presence of a finite chemical potential $\mu$ the ratio is reduced for fermions, because the degenerate particles do not carry entropy. However, even for large $\mu=2 T$ the ratio is still approximately 2.5 .

If quarks and antiquarks would simply recombine into mesons, mainly pions, there would be only 
half as many pions afterwards as there were quarks and antiquarks before: $N_{\pi}=\frac{1}{2}\left(N_{\mathrm{q}}+N_{\bar{q}}\right.$ ) (assuming $\mu=0$, for simplicity here). According to (4.3a) each pion carries 3.6 units of entropy (neglecting the pion mass: actual entropy per pion is near 4 for $m / T \gtrsim 1$ ), while each quark and antiquark carries 4.2 units of entropy. The entropy ratio between the hadronic (pion) gas and the qurk-gluon gas would therefore be

$$
\frac{s_{\pi}}{s_{\mathrm{q} \overline{\mathrm{q} g}}}=\frac{n_{\pi}}{n_{\mathrm{g}}+\left(\overline{n_{\mathrm{q}}}+n_{\overline{\mathrm{q}}}\right)^{\frac{7}{6}}} \sim \frac{1}{4}
$$

using the primordial gluon and quark densities

$$
\begin{aligned}
& n_{\mathrm{g}}=16\left(T^{3} / \pi^{2}\right) \zeta(3), \\
& n_{\mathrm{q}}+n_{\overline{\mathrm{q}}}=12\left(3 T^{3} / 2 \pi^{2}\right) \zeta(3) \quad(\mu=0),
\end{aligned}
$$

i.e.

$$
n_{\mathrm{g}}=\frac{8}{9}\left(n_{\mathrm{q}}+n_{\overline{\mathrm{q}}}\right)
$$

But contrary to eq. (4.4) we know that the total entropy should increase during the phase transition in view of the second law of thermodynamics. In order to appreciate what went wrong, we recall that the usual chemical reactions involve nonrelativistic particles, whose entropy per particle increases with temperature and also possibly on account of internal excitations. The entropy can, therefore, be made to increase while the number of particles decreases due to recombination. Such an effect is not possible for relativistic particles. The only practical way in which the factor 4 can be compensated is by allowing quarks and gluons to fragment into more quark-antiquark pairs before recombining into mesons. An alternate way would be to blow up the volume occupied by hadronic matter by a large factor, while $T$ and $\mu$ remain unchanged, but this would very likely be a highly endothermic process, impossible in the absence of a heat bath.

The large amount of entropy residing in the quark-gluon plasma can only be disposed of by generating a sufficiently large number of pions - this has been discussed at length in [GR85] and an estimate of $\mathrm{K}^{+} / \pi^{+} \sim 0.3-0.4$, i.e. 3 times hadronic reaction average and similar to the measured ratios in hadronic jets [KRa85] has been obtained. A tacit assumption is made here to the effect that all entropy resides in particle excitations in the plasma as well as in the hadronic phase. Therefore the possibility that the vacuum state itself may carry nonzero entropy or, more importantly, that there is a difference in entropy content between the perturbative and the true QCD vacuum is being disregarded. That this may occur is known e.g. from the electromagnetic Casimir effect where, under suitable conditions, the vacuum entropy may be a function of the boundary conditions (see e.g. [PMG85]). There is no indication at present that the modification of the vacuum has an important influence on the entropy balance, but it would be very interesting to see a more thorough study of this question.

There exists a simple mechanism permitting explicit implementation of the fragmentation process: recall that a quark-antiquark pair will only in one case out of nine be found in a colour singlet state that can form a single meson. In the other eight cases the quark-antiquark pair, forming a colour octet state, must first radiate a gluon in order to be able to recombine into a meson. Similarly, gluons will not, in general, simply disappear into the vacuum during the hadronization process in view of their colour 
octet nature, but will fragment into a quark-antiquark pair. We shall explore the consequences of this concept by considering the competition between the three processes (a) $q \bar{q} \rightarrow \pi$, (b) $q \bar{q} \rightarrow \pi G$, (c) $\mathrm{G} \rightarrow \mathrm{q} \overline{\mathrm{q}}$, shown diagrammatically in fig. 4.1. We shall denote the reaction rates for the three processes by $A, B, C$, respectively.

Then the following rate equations hold:

$$
\begin{aligned}
& \dot{N}_{\pi}=(A+B) N_{\mathrm{q}} N_{\overline{\mathrm{q}}} \\
& \dot{N}_{\mathrm{q}}=C N_{\mathrm{g}}-(A+B) N_{\mathrm{q}} N_{\overline{\mathrm{q}}} \\
& \dot{N}_{\overline{\mathrm{q}}}=C N_{\mathrm{g}}-(A+B) N_{\mathrm{q}} N_{\overline{\mathrm{q}}} \\
& \dot{N}_{\mathrm{g}}=-C N_{\mathrm{g}}+B N_{\mathrm{q}} N_{\overline{\mathrm{q}}} .
\end{aligned}
$$

Taking the difference between $(4.7 \mathrm{~b})$ and $(4.7 \mathrm{c})$ we immediately recover the baryon number conservation

$$
\dot{N}_{\mathrm{q}}-\dot{N}_{\overline{\mathrm{q}}}=0
$$

that is

$$
N_{\mathrm{q}}=N_{\overline{\mathrm{q}}}+b / 3
$$

where $b$ is the baryon number in plasma.

Adding $(4.7 \mathrm{c})$ and $(4.7 \mathrm{~d})$ we obtain the relation

$$
\dot{N}_{\bar{q}}+\dot{N}_{\mathrm{g}}=-A N_{\mathrm{q}} N_{\overline{\mathrm{q}}}
$$

which may be used to eliminate $N_{\bar{q}}$ from the right hand side of eq. (4.7a) to give

$$
\dot{N}_{\pi}=-(1+B / A)\left(\dot{N}_{\bar{q}}+\dot{N}_{\mathrm{g}}\right)
$$

that is

$$
N_{\pi}=-(1+B / A)\left(N_{\overline{\mathrm{q}}}+N_{\mathrm{g}}-N_{\overline{\mathrm{q}}}(0)-N_{\mathrm{g}}(0)\right) .
$$
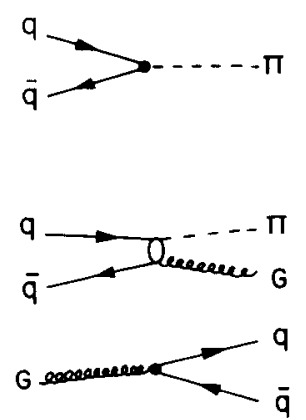
When the hadronization process is completed at $t=t_{\mathrm{h}}$, all antiquarks and gluons have been converted into mesons (here only pions) neglecting the rare formation of an antibaryon. We find, therefore,

$$
N_{\pi}\left(t_{\mathrm{h}}\right) \approx(1+B / A)\left(N_{\overline{\mathrm{q}}}(0)+N_{\mathrm{g}}(0)\right)
$$

However, requiring the condition $s_{\pi} \geq s_{\mathrm{q} \bar{q} g}$ in order to respect the second law of thermodynamics we expect (cf. eq. (4.4))

$$
N_{\pi}\left(t_{\text {had }}\right) \geq \frac{7}{3} N_{\mathrm{q}}(0)+N_{\mathrm{g}}(0) \quad(\mu=0) .
$$

Making use of eqs. (4.5) and (4.6) in order to relate $N_{\mathrm{q}}(0)$ and $N_{\mathrm{g}}(0)$, we obtain from eqs. (4.10) and (4.11) the condition

$$
B / A \geq \frac{12}{25} \sim \frac{1}{2} \quad(\mu=0) .
$$

We have thus shown that, in order to conserve entropy during the hadronization process, every gluon and about one third of the quarks must fragment before coalescing into mesons. Note that if the rates $A$ and $B$ were solely determined by colour weight factors we would have $B=8 A$. However, due to dynamical and kinematical reasons (diagram $4.1 \mathrm{~b}$ involves an additional factor $\alpha_{\mathrm{s}}$ and is phase-space suppressed) the rate $B$ can be expected to be considerably smaller than $8 A$. For $B / A=12 / 25$ at $\mu=0$ the entropy remains conserved in our model of the hadronization process; for higher ratios, the entropy increases during the phase transition. For future reference we also calculate the total number of fragmenting (participating) gluons $\tilde{N}_{\mathrm{g}}$ in the process represented by the diagrams of fig. 4.1. This number is defined as the difference between the final pion and the expected pion number if all primordial antiquarks recombined to form pions:

$$
\tilde{N}_{\mathrm{g}}=N_{\pi}-N_{\mathrm{q}}(0)=N_{\mathrm{g}}(0)+\frac{B}{A}\left(N_{\mathrm{q}}(0)+N_{\mathrm{g}}(0)\right)=\left(1+\frac{25}{16} \frac{B}{A}\right) N_{\mathrm{g}}(0) .
$$

Clearly, $\tilde{N}_{\mathrm{g}}>N_{\mathrm{g}}(0)$ and in view of eq. (4.12) the number of participating gluons is at least about twice as large as the number of primordial gluons when $\mu=0$.

At finite baryochemical potential the necessity for quark fragmentation is reduced, since baryon formation accounts for a significant fraction of the total entropy of the hadronic gas. On account of their large mass $M_{\mathrm{b}}$ the specific entropy of a baryon exceeds the value (4.3b). In the framework of the nonrelativistic approximation one finds

$$
(s / n)_{\text {baryon }} \approx 4+\left(M_{\mathrm{b}}-\mu_{\mathrm{b}}\right) / T
$$

where $\mu_{\mathrm{b}}=3 \mu_{\mathrm{q}}$ is the baryochemical potential. Although expression (4.14) assumes largest values for small $\mu_{\mathrm{b}}$, the net contribution of baryons to the total entropy grows with the baryon density which grows rapidly with $\mu_{\mathrm{b}}$, as $\exp \left[-\left(M_{\mathrm{b}}-\mu_{\mathrm{b}}\right) / T\right]$. Numerical studies show explicitly that the need for quark fragmentation in order to conserve entropy in the process of phase transition (or transformation) quickly decreases as $\mu_{\mathrm{b}}$ grows. Thus at high net baryon density $\rho>\rho_{0}$ it is sufficient in our model calculations to allow only for fragmentation of those gluons which are initially present in the plasma phase, i.e. to put $\tilde{N}_{\mathrm{g}}=N_{\mathrm{g}}$. 


\subsection{Combinatoric break-up model}

Having accounted for entropy conservation and possible entropy generation in the hadronization process, we have determined which portions of the quarks and antiquarks contained in the mesons and baryons after the quark-gluon plasma break-up are remnants of the previously existing (constituent) particles and which are newly produced by fragmentation. When we now study the flavour composition of the hadrons created in the break-up, we must first determine the flavour content both of the constituent quarks and antiquarks and of those produced by fragmenting gluons.

The flavour composition of the quark-gluon plasma at equilibrium and the approach to it has been discussed in detail in the previous sections. We shall assume that the flavour content of the quark constituents of the plasma are just those obtained from integration of the rate equation up to the time of break-up. When the break-up occurs gradually via an intermediate mixed phase, then a different flavour distribution will have to be used at each instant of time according to the evolution equations. However, we will not consider possible re-dissolution of hadrons into the quark-gluon phase through the boundary between the two phases. We thus view the break-up process as proceeding in a single direction: quarks coalesce to form hadrons but not vice versa.

In order to determine the flavour content of the quark-antiquark pairs produced by fragmentation, further theoretical or experimental information is required. Fortunately, flavour dependence of quark jet fragmentation has been extensively studied both experimentally and theoretically. Although it is not entirely clear whether the concepts developed for jet fragmentation are valid in the environment of the hadronizing quark-gluon plasma, we shall adopt these concepts as a first guideline. In particular, it has been shown in the context of the flux-tube model [CNN79, GM83] that the relative probability of glue fragmentation into a quark pair of mass $m_{i}$ is controlled by the parameter

$$
f_{i}=N \exp \left(-m_{i}^{2} / \kappa\right)
$$

where $\kappa \sim 1 \mathrm{GeV} / \mathrm{fm}$ is the QCD string constant. These values have been used in stochastic quark jet fragmentation models [FF78, AGIS83] and were shown to provide a successful description of the experimental data. For light quarks we can take $m_{\mathrm{u}}=m_{\mathrm{d}}=0$, so that the normalization constant becomes

$$
N^{-1}=2+\exp \left(-m_{\mathrm{s}}^{2} / \kappa\right)
$$

leading to $f_{\mathrm{u}}=f_{\mathrm{d}} \sim 0.425, f_{\mathrm{s}} \sim 0.15$ for $m_{\mathrm{s}} \sim 170 \mathrm{MeV}$. We shall not consider fragmentation into heavier quark flavours.

The number of quark-antiquark pairs of each flavour is now obtained by multiplying the effective number of fragmenting gluons $\tilde{N}_{\mathrm{g}}$, given by eq. (4.14), with the probability $f_{i}$ for producing the considered flavour.

The flavour composition of all the quarks and antiquarks that finally become constituents of the hadrons produced in the break-up of the plasma is now fully determined. At a given time there are for each flavour, the primary quarks $\mathrm{N}_{i}$ or antiquarks $\overline{\mathrm{N}}_{i}$ created by the quarko-chemical reactions, and there are those generated by glue fragmentation. When we combine these, we obtain the following expressions from the numbers of quarks and antiquarks of each flavour that effectively contribute to hadronization:

$$
\tilde{N}_{\mathrm{q}}=N_{\mathrm{q}}+f_{\mathrm{q}} \tilde{N}_{\mathrm{g}}, \quad \tilde{N}_{\overline{\mathrm{q}}}=N_{\overline{\mathrm{q}}}+f_{\mathrm{q}} \tilde{N}_{\mathrm{g}}
$$




$$
\tilde{N}_{\mathrm{s}}=\tilde{N}_{\overline{\mathrm{s}}}=N_{\mathrm{s}}+f_{\mathrm{s}} \tilde{N}_{\mathrm{g}}
$$

where

$$
N_{\mathrm{q}}=N_{\mathrm{u}}+N_{\mathrm{d}}, \quad f_{\mathrm{q}}=f_{\mathrm{u}}+f_{\mathrm{d}} .
$$

$N_{\mathrm{q}}$ will be taken at the (chemical) equilibrium value, but $N_{\mathrm{s}}$ must be determined by integration of the strangeness rate equations discussed in section 3 , should it be different from equilibrium value in a particular case considered.

After the development of the gluon fragmentation we can now apply the combinatoric break-up model to determine the flavour composition of the hadrons at the beginning of the evolution of the final hadronic phase. In the framework of this combinatoric model, the available quarks are distributed among the final state hadrons by using two constant probabilities: one for meson production and one for baryon production. The abundance of mesons is assumed to be related to quark abundances as follows:

$$
\begin{aligned}
& N_{\pi}=\alpha \tilde{N}_{\mathrm{q}} \tilde{N}_{\overline{\mathrm{q}}}, \\
& N_{\mathrm{K}}=\alpha \tilde{N}_{\mathrm{q}} \tilde{N}_{\overline{\mathrm{s}}}, \quad N_{\overline{\mathrm{K}}}=\alpha \tilde{N}_{\mathrm{s}} \tilde{N}_{\overline{\mathrm{q}}}, \\
& N_{\phi}=\alpha \tilde{N}_{\mathrm{s}} \tilde{N}_{\overline{\mathrm{s}}},
\end{aligned}
$$

where $\alpha$ is a recombination constant characterising the formation of mesons. The abundance of baryons is similarly given by

$$
\begin{array}{ll}
N_{\mathrm{N}}=\frac{1}{3 !} \beta \tilde{N}_{\mathrm{q}}^{3}, & N_{\overline{\mathrm{N}}}=\frac{1}{3 !} \beta \tilde{N}_{\overline{\mathrm{q}}}^{3}, \\
N_{\mathrm{Y}}=\frac{1}{2 !} \beta \tilde{N}_{\mathrm{q}}^{2} \tilde{N}_{\mathrm{s}}, & N_{\overline{\mathrm{Y}}}=\frac{1}{2 !} \beta \tilde{N}_{\overline{\mathrm{q}}}^{2} \tilde{N}_{\overline{\mathrm{s}}}, \\
N_{\Xi}=\frac{1}{2 !} \beta \tilde{N}_{\mathrm{q}} \tilde{N}_{\mathrm{s}}^{2}, & N_{\bar{\Xi}}=\frac{1}{2 !} \beta \tilde{N}_{\overline{\mathrm{q}}} \tilde{N}_{\overline{\mathrm{s}}}^{2}, \\
N_{\Omega}=\frac{1}{3 !} \beta \tilde{N}_{\mathrm{s}}^{3}, & N_{\bar{\Omega}}=\frac{1}{3 !} \beta N_{\overline{\mathrm{s}}}^{3},
\end{array}
$$

and $\beta$ is the recombination constant characterising the formation of baryons. The various quarks are distributed among the hadrons subject to the conservation laws for each flavour:

$$
\begin{aligned}
& \tilde{N}_{\mathrm{q}}=N_{\pi}+N_{\mathrm{K}}+3 N_{\mathrm{N}}+2 N_{\mathrm{Y}}+N_{\Xi}, \\
& \tilde{N}_{\overline{\mathrm{q}}}=N_{\pi}+N_{\overline{\mathrm{K}}}+3 N_{\overline{\mathrm{N}}}+2 N_{\overline{\mathrm{Y}}}+N_{\bar{\Xi}}, \\
& \tilde{N}_{\mathrm{s}}=N_{\overline{\mathrm{K}}}+N_{\phi}+N_{\mathrm{Y}}+2 N_{\Xi}+3 N_{\Omega}, \\
& \tilde{N}_{\overline{\mathrm{s}}}=N_{\mathrm{K}}+N_{\phi}+N_{\overline{\mathrm{Y}}}+2 N_{\Xi}+3 N_{\bar{\Omega}} .
\end{aligned}
$$


When eqs. (4.17) and (4.18) are inserted into these conservation laws, the four equations reduce to two identical sets of two equations each for the coefficients $\alpha$ and $\beta$ :

$$
\begin{aligned}
& \alpha \bar{Q}+\frac{1}{2} \beta Q^{2}=1, \\
& \alpha Q+\frac{1}{2} \beta \bar{Q}^{2}=1,
\end{aligned}
$$

with the abbreviations

$$
Q=\tilde{N}_{\mathrm{q}}+\tilde{N}_{\mathrm{s}}, \quad \bar{Q}=\tilde{N}_{\overline{\mathrm{q}}}+\tilde{N}_{\overline{\mathrm{s}}} .
$$

Equation (4.20) leads to:

$$
\begin{aligned}
& \alpha=\frac{Q+\bar{Q}}{Q^{2}+Q \bar{Q}+\bar{Q}^{2}}=\frac{4(Q+\bar{Q})}{3(Q+\bar{Q})^{2}+(Q-\bar{Q})^{2}}, \\
& \beta=\frac{2}{Q^{2}+Q \bar{Q}+\bar{Q}^{2}}=\frac{8}{3(Q+\bar{Q})^{2}+(Q-\bar{Q})^{2}} .
\end{aligned}
$$

In view of strangeness conservation we have $N_{\mathrm{s}}=N_{\tilde{\mathrm{s}}}$, and therefore

$$
\begin{aligned}
& Q+\bar{Q}=\bar{N}_{\mathrm{q}}+\tilde{N}_{\overline{\mathrm{q}}}+2 \tilde{N}_{\mathrm{s}} \\
& Q-\bar{Q}=\tilde{N}_{\mathrm{q}}-\tilde{N}_{\overline{\mathrm{q}}} .
\end{aligned}
$$

As the pion differs from the other mesons due to its double role as member of the meson multiplet and as Goldstone boson of chiral symmetry breaking, it might be tempting to replace the expression $N_{\pi}=\alpha \tilde{N}_{\mathrm{q}} \tilde{N}_{\overline{\mathrm{q}}}$ in eq. (4.17) by an analogous formula with an independent parameter $\alpha_{\pi}$ :

$$
N_{\pi}=\alpha_{\pi} \tilde{N}_{\mathrm{q}} \tilde{N}_{\overline{\mathrm{q}}}
$$

It turns out, however, that the balance equations (4.19) require $\alpha_{\pi}=\alpha$, otherwise they are inconsistent.

\subsection{Hadrons from quark-gluon plasma}

We shall now explore the predictions of the recombination-fragmentation model for the chemical composition of hadronic matter immediately after the hadronization process, if we assume chemical (and thermal) equilibrium in the quark-gluon phase.

The primordial $\mathrm{q}, \overline{\mathrm{q}}$ and $\mathrm{s}=\overline{\mathrm{s}}$ abundances are generally assumed to be given by their respective equilibrium values (we kept the first 5 terms in the series expansion below):

$$
\rho_{\mathrm{s}}=\rho_{\overline{\mathrm{s}}} \simeq \frac{3 T^{3}}{\pi^{2}}\left(\frac{m_{\mathrm{s}}}{T}\right)^{2} \sum_{n} \frac{(-)^{n-1}}{n} K_{2}\left(\frac{n m_{\mathrm{s}}}{T}\right)
$$




$$
\begin{aligned}
& \rho_{\overline{\mathrm{q}}} \simeq \frac{6 T^{3}}{\pi^{2}} \sum_{n} \frac{(-)^{n-1}}{n} \exp \left(-n \mu_{\mathrm{b}} / 3 T\right) \\
& \rho_{\mathrm{q}}-\rho_{\overline{\mathrm{q}}}=\frac{T^{3}}{\pi^{2}}\left\{\left(\frac{\mu_{\mathrm{b}}}{3 T}\right)^{3}+\pi^{2}\left(\frac{\mu_{\mathrm{b}}}{3 T}\right)\right\} \\
& \rho_{\mathrm{q}}=\rho_{\overline{\mathrm{q}}}\left[\left(\rho_{\mathrm{q}}-\rho_{\overline{\mathrm{q}}}\right) / \rho_{\overline{\mathrm{q}}}+1\right]
\end{aligned}
$$

which are to be multiplied by the volume to give $N_{\mathrm{q}}, N_{\overline{\mathrm{q}}}, N_{\mathrm{s}}=N_{\overline{\mathrm{s}}}$. To find the active quark numbers $\tilde{N}_{\mathrm{q}}, \tilde{N}_{\overline{\mathrm{q}}}, \tilde{N}_{\mathrm{s}}=\tilde{N}_{\overline{\mathrm{s}}}$ according to eqs. (4.16) we also need the density of gluons, i.e.

$$
\rho_{\mathrm{g}}=16\left(T^{3} / \pi^{2}\right) \zeta(3)
$$

For the purpose of the numerical study of the importance of fragmentation, it is convenient to write, instead of eq. (4.16)

$$
\tilde{N}_{\mathrm{q}}=N_{\mathrm{q}}+f_{\mathrm{q}} N_{\mathrm{g}}+f_{\mathrm{q}}^{\prime}\left(N_{\mathrm{q}}+N_{\overline{\mathrm{q}}}\right)
$$

same equation for light antiquarks and

$$
\tilde{N}_{\mathrm{s}}=\tilde{N}_{\overline{\mathrm{s}}}=N_{\mathrm{s}}+f_{\mathrm{s}} N_{\mathrm{g}}
$$

\begin{tabular}{|c|c|c|c|}
\hline Curve & $f_{\mathrm{q}}$ & $f_{\mathbf{q}}^{\prime}$ & Comments (fig. 4.2) \\
\hline (a) & 0 & 0 & No fragmentation \\
\hline (b) & 0.85 & 0 & Only gluon fragmentation \\
\hline (c) & $0.85 / 2$ & $1 / 2$ & $\begin{array}{l}\text { Reduced gluon fragmentation } \\
\text { and some quark fragmentation }\end{array}$ \\
\hline (d) & 0 & 1 & Only quark fragmentation \\
\hline (e) & 0.85 & 1 & $\begin{array}{l}\text { Fragmentation of quarks and } \\
\text { gluons, quarks fragment stronger } \\
\text { than gluons }\end{array}$ \\
\hline
\end{tabular}

Light quark fragmentation to strange quarks has been neglected here. In this way we can explicitly explore the consequences of the fragmentation of both gluons and quarks which is not anymore possible in eq. (4.16). $f_{\mathrm{q}}$ has the same meaning as previously, while $f_{\mathrm{q}}^{\prime}$ is a quark fragmentation probability parameter. We denote in fig. 4.2 the curves as:

$f_{\mathrm{s}}=0.15$ in all instances, i.e. there is a small glue fragmentation into strange quarks.

In fig. 4.2 the ratio of quark-gluon plasma to hadronic gas of 'meson per baryon' is shown along the critical curve separating the phases, see section 1.4 . In the quark-gluon plasma hadronization we have

$$
\begin{aligned}
& \rho^{\mathrm{QGP}}(\mathrm{q} \bar{q})=\alpha \tilde{q} \overline{\bar{q}} \\
& \rho_{\mathrm{B}}^{\mathrm{QGP}}=(q-\bar{q}) / 3
\end{aligned}
$$

while in the hadronic gas 


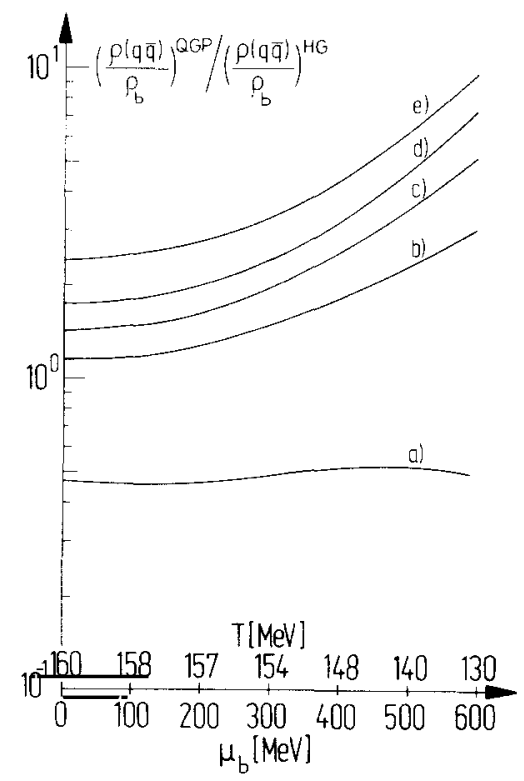

Fig. 4.2. Mesons per baryon along the assumed critical curve, both $\left(T_{\mathrm{cr}}, \mu_{\mathrm{cr}}\right)$ are given. Ratio of quark-gluon plasma recombination to hadronic gas abundances is shown. Curve (a) excludes fragmentation, while (b)-(e) include different degrees of fragmentation (see table in text for fragmentation parameters).

$$
\begin{aligned}
& \rho^{\mathrm{HG}}(\mathrm{q} \overline{\mathrm{q}})=\rho_{\pi}+\rho_{\eta}+\rho_{\eta^{\prime}}+\rho_{\rho}+\rho_{\omega}+\rho_{\mathrm{\delta}} \\
& \rho_{\mathrm{B}}^{\mathrm{HG}}=\rho_{\mathrm{N}}+\rho_{\Delta}+\rho_{\mathrm{Y}}+\rho_{\Xi}+\rho_{\Omega}-\text { antibaryons } .
\end{aligned}
$$

We see in fig. 4.2 that without fragmentation, considering only recombination, the 'meson per baryon' function along the assumed critical curve is smaller in quark-gluon plasma than in hadronic gas phase. The introduction of fragmentation permits that there are more mesons per baryons in plasma than in the corresponding hadronic gas phase. It is hard to decide which of the fragmentation curves (b) - (e) best reflect on the actual physical behaviour of hadronising plasma. With fragmentation permitted, we find that particle multiplicity from quark-gluon plasma can easily be three times higher than if no plasma were formed, in agreement with qualitative estimates based on entropy arguments. But the real strength of our approach is only apparent once strange quarks are involved.

We first consider the degree of clustering of $s-\bar{s}$ quarks in hadronising plasma along the critical curve, cf. fig. 4.3. Here we use

$$
\begin{aligned}
& \rho(\mathrm{s} \bar{s})=\alpha \tilde{s} \bar{s} \\
& \rho(\mathrm{q} \bar{s})+\rho(\overline{\mathrm{q}} \mathrm{s})=\alpha(\tilde{\bar{s}} \tilde{q}+\tilde{\bar{q}} \tilde{s})
\end{aligned}
$$

and hence $(\tilde{\bar{s}}=\tilde{s})$ :

$$
\frac{\rho(\mathrm{ss})}{\rho(\mathrm{qs})+\rho(\overline{\mathrm{q}} \mathrm{s})}=\frac{\tilde{s}}{\tilde{q}+\tilde{\bar{q}}} .
$$




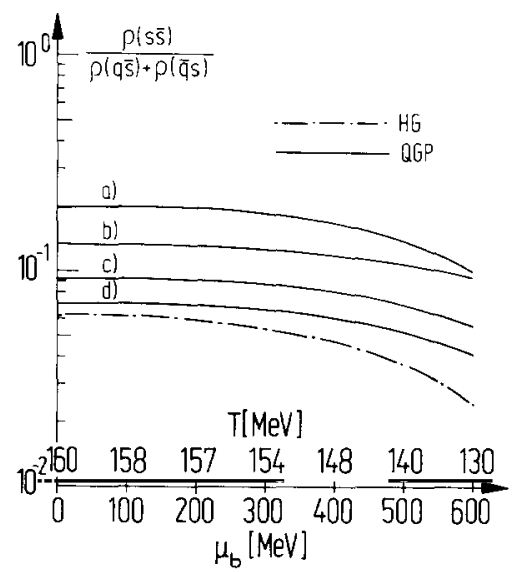

Fig. 4.3. ss̄ clustering: Full lines quark-gluon plasma recombination results. (a) without fragmentation. (b)-(d) including fragmentation (see table in text for choice of parameters). Dashed-dotted are hadronic gas equilibrium results.

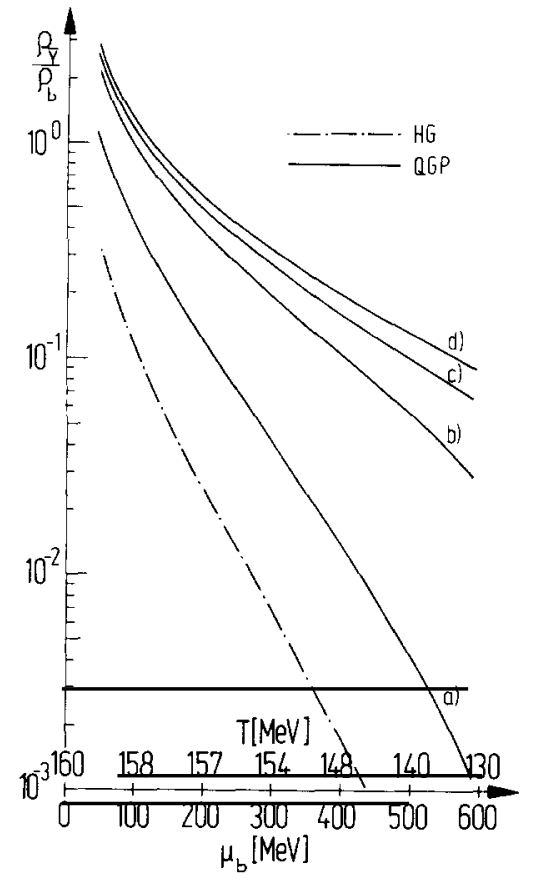

Fig. 4.4. Antihyperon abundance along the assumed critical curve, full lines recombination from qurk-gluon plasma, (b)-(d) including fragmentation. Dashed-dotted are hadronic gas equilibrium results.

The dashed-dotted line in fig. 4.3 is the hadronic gas result using:

$$
\begin{aligned}
& \rho_{\mathrm{s} \overline{\mathrm{s}}}^{\mathrm{HG}}=\rho_{\phi}+\rho_{\mathrm{S}^{*}} \\
& \rho_{\mathrm{s} \overline{\mathrm{q}}}^{\mathrm{HG}}+\rho_{\overline{\mathrm{s}} \mathrm{q}}=\rho_{\mathrm{K}}+\rho_{\overline{\mathrm{K}}}+\rho_{\mathrm{K}^{*}}+\rho_{\overline{\mathrm{K}}^{*}} .
\end{aligned}
$$

We note that the curve marked (d) is very close to the hadronic gas result. It corresponds to permitted fragmentation of quarks and gluons. As before (a) has only recombination and it then leads to prediction of substantial sis clustering, which is diluted by the fragmentation process. Specifically, the notation is in figs. 4.3 and 4.4 below:

\begin{tabular}{llll}
\hline Curve & $f_{\mathrm{q}}$ & $f_{\mathrm{q}}^{\prime}$ & Comments (figs. 4.3 and 4.4) \\
\hline (a) & 0 & 0 & Only recombination \\
(b) & 0.85 & 0 & Fragmentation of gluons \\
(c) & 0.85 & 0.5 & Fragmentation of quarks \& gluons \\
(d) & 0.85 & 1 & $\begin{array}{l}\text { Fragmentation of quarks \& gluons, } \\
\text { quarks fragment stronger than gluons }\end{array}$ \\
\hline
\end{tabular}

Thus we find quite abundant formation of $\bar{s}\left(\phi, S^{*}\right)$ in any scenario of nuclear reaction, with 5-20\% of all strangeness found in these hadronic states. In this regard we agree with earlier suggestions (see Shor 
[Sh85] and references therein) that abundantly produced $\phi$-mesons are indicative for the plasma state formation. However, as the presented calculations show, the total $\phi$ abundance will not exceed the hadronic gas equilibrium value significantly. Since a $\phi$-meson is nearly a virtual $\mathrm{K}-\overline{\mathrm{K}}$ cluster, we expect that rapid strangeness exchange reactions on baryons will rapidly re-establish hadronic gas equilibrium expectations. In that observation we differ significantly from conjectures presented recently [Sh85] that $\phi$-mesons will escape easily from the fireball due to their small 'size'. We must allow for resonant strangeness exchange reactions on baryons, leading to rapid equilibration of the $\phi$-meson population.

In fig. 4.4 we show the abundance of antihyperons per baryon resulting from the hadronisation, along the critical curve. We use

$$
\rho_{\overline{\mathrm{Y}}}^{\mathrm{QGP}}=\frac{\beta}{2 !} \tilde{q}^{2 \tilde{\bar{S}}}
$$

and $\rho_{\mathrm{B}}^{\mathrm{OGP}}$ as in eq. (4.27b). Dashed-dotted is the hadronic gas result. As the fragmentation provides additional antiquarks we are seeing that fragmentation curves (b), (c), (d), lead to very substantial abundance of antihyperons nearly two orders of magnitude above the already substantial (normally unattainable) equilibrium abundance expected in hadronic gas (dashed-dotted curve). We will discuss the method of computing hadronic gas equilibrium abundance, in detail, further below in section 6 and
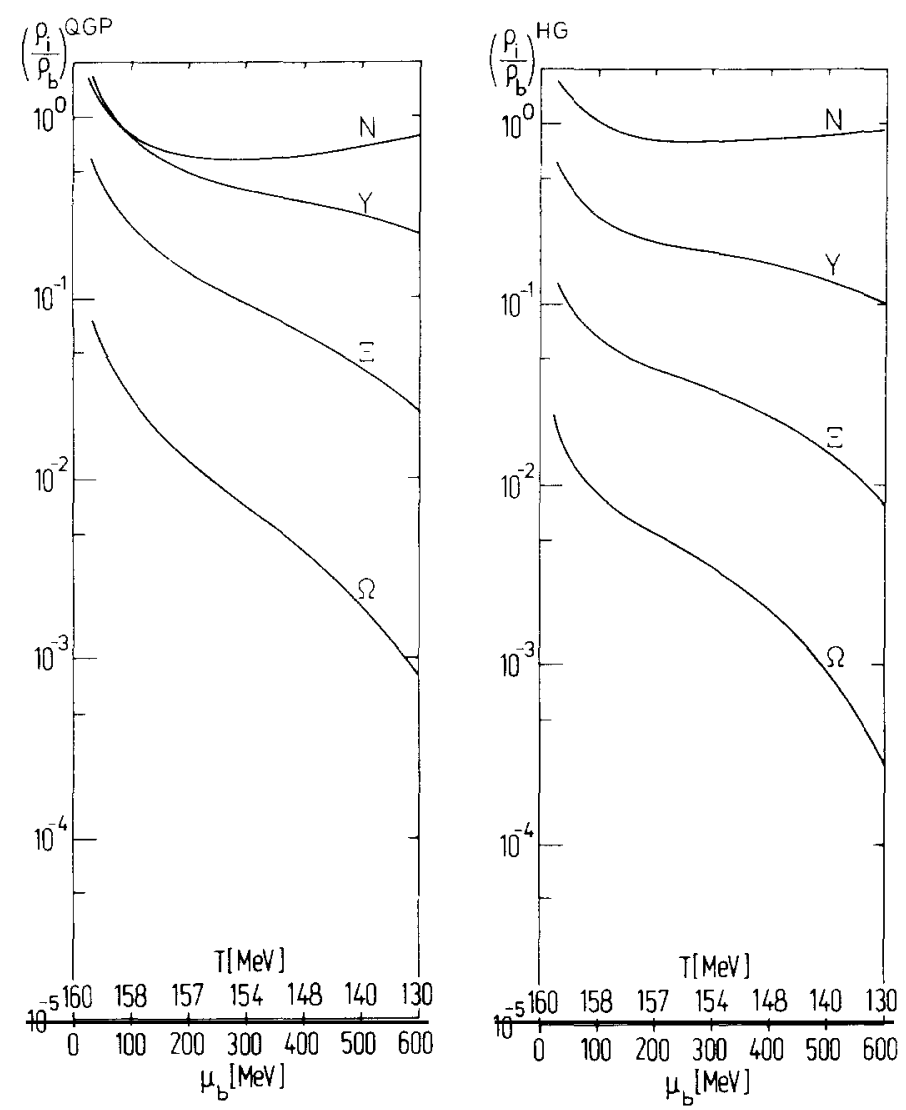

Fig. 4.5. Baryon abundance from quark-gluon recombination without fragmentation along critical curve. Quark-gluon plasma and equilibrium hadronic gas are shown separately. 
note here, however, that we should not expect that equilibrium abundance is reached at all without the existence of a plasma source of antihyperons (see section 5).

It is very important to appreciate the result that for each baryon we expect one tenth or more antihyperons. Even though this number may be depleted by annihilation reactions (see section 7), it is less depleted than the number of antinucleons and hence the cornerstone of the proposal is established that $\bar{Y} / \bar{N}$ ratio is a characteristic observable of the quark-gluon plasma [Ra81-84].

These considerations make clear that antibaryon production from the quark-gluon plasma cannot be reliably predicted without proper treatment of entropy conservation through gluon fragmentation. The suggestion by Greiner and coworkers [HSG84, He85] that antinucleon and even antinuclei formation could be a signature of the quark-gluon plasma is based on a description which totally neglects this effect. (We shall return to this point in section 7.3 when we discuss results of a full-scale calculation of quark-gluon plasma expansion, cooling and hadronization.)

Mainly as a matter of record we show in figs. 4.5 and 4.6 baryon and resp. antibaryon abundance in the recombination model without fragmentation along the critical line and compare it with the equilibrium hadronic gas. As we shall study in detail below, antibaryons in hadronic gas must be expected to always be far below the equilibrium abundance except if quark-gluon plasma is formed. Notable is the enhancement of $\bar{\Omega}$ abundance even without fragmentation. As seen in fig. 4.4,
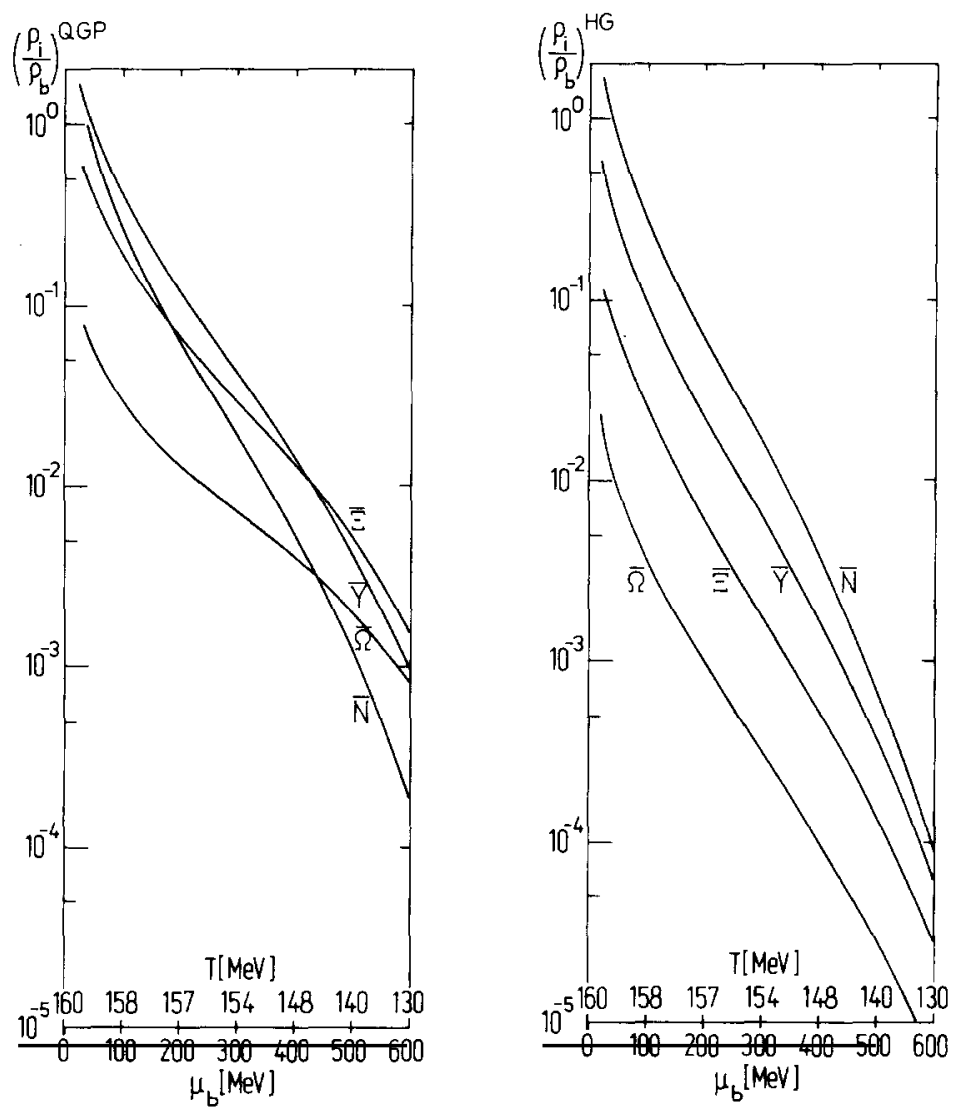

Fig. 4.6. Antibaryon abundance from quark-gluon recombination without fragmentation along critical curve. Quark-gluon plasma and equilibrium hadronic gas are shown separately. 
fragmentation will enhance antibaryon abundance as more antiquarks become disposable to form antibaryons. Thus we can conclude this section with the observation that a substantial enhancement is expected for antibaryon abundances arising from the plasma state, even when compared to the equilibrium abundance of the hadronic gas. It is predicted that from a baryon-rich plasma antihyperons will be initially produced quite abundantly, at the level of 0.1 per baryon (cf. fig. 4.4). We return to discuss the evolution of these abundances in expanding fireballs in section 7.

Kämpfer et al. [Ka85] have modified the recombination model to account for the available phase space of the produced hadrons, which depends on the mass $m_{i}$ of the hadronic state. They find that the entropy of the hadron gas is maximised, given a fixed number of hadronic constituents, when the particle numbers obtained from the recombination model (without fragmentation) are multiplied by Boltzmann factors

$$
f_{i}=\exp \left(-m_{i} / T\right)
$$

The introduction of these weights amounts to the assumption that thermal and chemical equilibrium is reached instantaneously in the hadron phase, as far as it is allowed by the limitations from the availability of constituent quarks and antiquarks.

When the factors $f_{i}$ are included, the expected composition of the hadronic gas phase becomes much closer to that at chemical equilibrium, which will be discussed in section 6 (see fig. 6.2). Figure 4.7 shows the results obtained by Kämpfer et al., not accounting for fragmentation processes. We shall test the validity of the assumption underlying the factors (4.32) in section 7.2 . There we treat the temporal

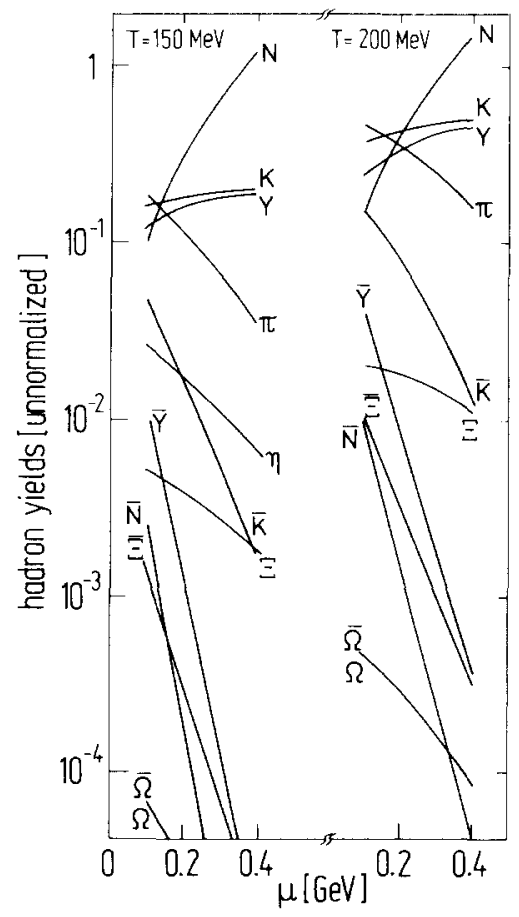

Fig. 4.7. Unnormalized hadron yields versus quark chemical potential $\left(\mu=\mu_{\mathrm{b}} / 3\right)$ at freeze-out, for two different temperatures. The model assumes the validity of weight factors $f_{i}=\exp \left(-m_{i} / T\right)$, but no fragmentation (from [Ka85]). 
evolution of the composition of the hadronic gas phase when it is still in equilibrium with the quark-gluon plasma. We shall find then that relative equilibrium is reached only when the coexistence period is very long, certainly longer than $20 \mathrm{fm} / c$. In practical cases, therefore, the introduction of weight factors like (4.32) must be regarded with considerable doubt.

\section{Strange hadrons in hadronic gas - Evolution and formation}

\subsection{Relevant reactions}

We now study the dynamics of both strangeness production and redistribution in the hadronic gas phase and proceed to compute the timescales involved in strangeness production and exchange reactions, with particular emphasis put on the case of multiple strangeness carrying antibaryons [KR85]. A scenario will be assumed in which there is no quark-gluon plasma formed. However, the set of evolution equations we develop for the strange particle evolution, in the spirit of chemical reaction (kinetic) equations [Me82] is valid under all assumed reaction scenarios as only the initial conditions need to be modified from case to case. The purpose of this section is to estimate the strange particle abundances expected if no quark-gluon plasma has been found.

At the stage of the nuclear reaction when a thermally equilibrated fireball has been established, the standard reaction for strangeness production

$$
\mathrm{N}+\mathrm{N} \rightarrow \mathrm{N}+\mathrm{Y}+\mathrm{K}
$$

where $\mathrm{Y}=\Lambda$ or $\Sigma$, play almost no role, because: (1) its threshold at $\sim 670 \mathrm{MeV}$ energy in the centre-of-mass frame is higher than in reactions between a pion and a baryon, or between two pions, (2) the pions are the most abundant particles except perhaps at very high values of $\mu_{\mathrm{b}}$, (3) the phase space of two-particle final states is more favourable than that of three-body final states. In consequence, the following strangeness producing reactions were considered by Koch and Rafelski [KR85]:

$$
\begin{array}{lll}
\pi+\mathrm{N} \rightarrow \mathrm{K}+\mathrm{Y} & \pi+\overline{\mathrm{N}} \rightarrow \overline{\mathrm{K}}+\overline{\mathrm{Y}} & \pi+\pi \rightarrow \mathrm{K}+\overline{\mathrm{K}} \\
\pi+\mathrm{Y} \rightarrow \Xi+\mathrm{K} & \pi+\overline{\mathrm{Y}} \rightarrow \overline{\mathrm{K}}+\bar{\Xi} \\
\pi+\Xi \rightarrow \Omega+\mathrm{K} & \pi+\bar{\Xi} \rightarrow \overline{\mathrm{K}}+\bar{\Omega} .
\end{array}
$$

The common reaction feature of all processes listed under (5.1) is the $q \bar{q} \rightarrow s \bar{s}$ reaction, see fig. 5.1, where three quarks are spectators, and a $\mathrm{q} \overline{\mathrm{q}}$ pair is annihilated and an $\bar{s}$ pair is formed. The experimental value of the OZI-rule forbidden cross section for the reaction $\pi+N \rightarrow Y+K(N \cong$ nucleon), is only about $0.1 \mathrm{mb}$ in the energy region of interest. Hence, the strangeness phase space saturation in $\mathrm{HG}$ should be a relatively slow process since the sis forming collision time of a pion with a nucleon, at $\rho \sim 3 \rho_{0}=\frac{1}{2} / \mathrm{fm}^{3}$ is

$$
\tau \sim(\sigma v \rho)^{-1} \sim\left(10^{-2} \mathrm{fm}^{2} \cdot 3 \times 10^{23} \mathrm{fm} / \mathrm{s} \cdot \frac{1}{2} / \mathrm{fm}^{3}\right)^{-1} \sim 10^{-21} \mathrm{~s} .
$$

It is also clear that direct pair production of multiple strangeness carrying (anti-)baryons is strongly 

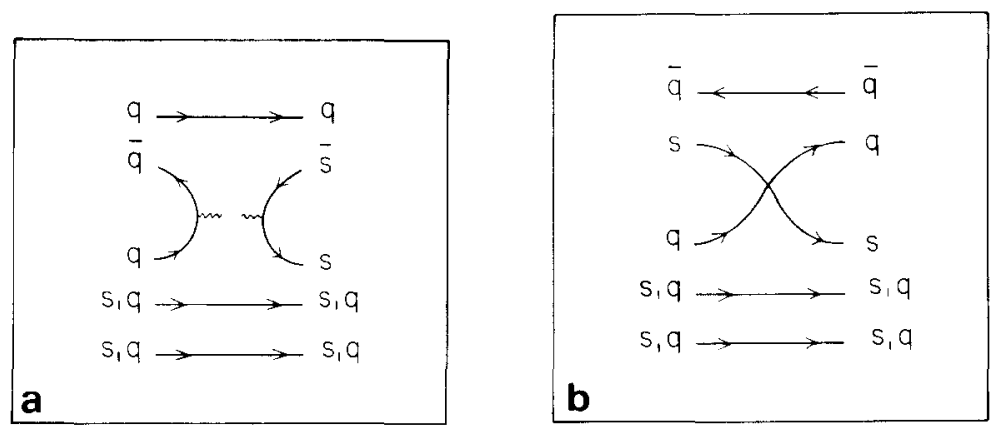

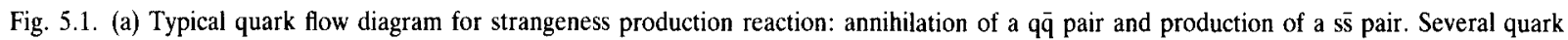
spectator lines are also indicated. (b) Typical quark flow diagram for strangeness exchange reaction: exchange of the s-quark from the initial $\mathrm{K}$-meson to the final baryon. Several quark spectator lines are also indicated.

suppressed in nuclear collisions. But these particles may be produced by the following strange quark exchange reactions:

$$
\begin{array}{ll}
\overline{\mathrm{K}}+\mathrm{N} \rightarrow \mathrm{Y}+\pi & \mathrm{K}+\overline{\mathrm{N}} \rightarrow \overline{\mathrm{Y}}+\pi \\
\overline{\mathrm{K}}+\mathrm{Y} \rightarrow \Xi+\pi & \mathrm{K}+\overline{\mathrm{Y}} \rightarrow \bar{\Xi}+\pi \\
\overline{\mathrm{K}}+\Xi \rightarrow \Omega+\pi & \mathrm{K}+\bar{\Xi} \rightarrow \bar{\Omega}+\pi .
\end{array}
$$

All these reactions are 'exothermic' as the reaction products are less massive than the particles in the initial state. Experimentally, one finds for the strangeness exchange reaction $\bar{K}+\mathrm{N} \rightarrow \mathrm{Y}+\pi$ a cross section roughly ten times larger than the production cross section eq. (5.1). This means that strangeness is much faster redistributed among the strange particle family than produced. The underlying type of subprocess for all reactions in eq. (5.2) is depicted in fig. 5.2 where the strange quark from a kaon is exchanged to the baryon and forms a hyperon $\mathrm{Y}$.

Fast redistribution of strange s-quarks among different hadrons is required for the relative chemical equilibrium of s-quarks to be reached during the lifetime of the fireball. It is worth mentioning that the exchange processes often proceed via resonant intermediate states such as $\bar{K}+N \rightarrow Y_{\text {res }} \rightarrow \mathrm{Y}+\pi$. This effect is included in the dynamical approach described here in that the resonance cross sections for the exchange reactions are being used. What is neglected is the possibility of the intermediate resonant state reacting before decay. This effect along with other effects such as, e.g. a possible increase in effective hadronic size leading to an increase of the exchange reaction cross sections, is not taken into account.

As emphasised in eq. (5.2) the generation of strange antibaryons proceeds via antinucleons which, however, have a very low abundance density in the hadronic phase. This is a consequence of the small production cross sections for antinucleons in combination with the strength of $\mathrm{NN}$ annihilations into multiple pion final states. Consider e.g. the annihilation reaction: the energies below $1 \mathrm{GeV}$ the $p+\bar{p} \rightarrow 5 \pi$ cross section is approximately 100 times larger than the strangeness production cross section via pions. Thus even though the (unsaturated) phase space density of antinucleons is quite low, it is essential to consider the following set of annihilation reactions in our calculations in order to account for the competition of the strangeness exchange, reaction (5.2), with annihilation reactions: 


$$
\begin{array}{ll}
\mathrm{N}+\overline{\mathrm{N}} \rightarrow \sim 5 \pi, & \\
\mathrm{Y}+\overline{\mathrm{N}} \rightarrow \overline{\mathrm{K}}+\sim 4 \pi, & \overline{\mathrm{Y}}+\mathrm{N} \rightarrow \mathrm{K}+\sim 4 \pi, \\
\Xi+\overline{\mathrm{N}} \rightarrow 2 \overline{\mathrm{K}}+\sim 3 \pi, & \bar{\Xi}+\mathrm{N} \rightarrow 2 \mathrm{~K}+\sim 3 \pi, \\
\Omega+\overline{\mathrm{N}} \rightarrow 3 \overline{\mathrm{K}}+\sim 2 \pi, & \bar{\Omega}+\mathrm{N} \rightarrow 3 \mathrm{~K}+\sim 2 \pi .
\end{array}
$$

So far, in this context, all the annihilation strength has been concentrated in the five-body final state because this channel is favoured by phase space arguments. Adding a mean thermal energy to the rest masses of all particles in the final channel, we find to a good approximation the energy of the incoming baryon-antibaryon channel. It is well known that phase space can effectively suppress low energy many-body final channels and favours 'thermal' production modes [Ha65]. Also annihilation between two strange baryons has been neglected, because in this case both participants have a suppressed phase space density. It is known from experimental study of $\mathrm{p} \overline{\mathrm{p}}$ annihilation that the multiple pion final state is often produced via intermediate resonance states, such as $\rho \rho \pi, \omega \pi \pi$, etc. The open issue here is again whether these intermediate resonances could possibly participate in further reactions before they decay.

As only a selected number of all accessible hadronic states have been included in the above considerations, one may wonder if the inclusion of the higher-lying hadrons does not essentially alter the picture. While the number densities of particles in statistical equilibrium get exponentially smaller with their mass increasing, the number of states also increases exponentially. However, in a short-lived system such as formed in high energy nuclear collisions tails of mass distributions will hardly be populated (except perhaps in the case of quark-gluon plasma) and only a limited number of hadronic resonances will be excited. Including the kinetic equations, all low-lying hadronic states, and using resonant cross sections seems therefore an entirely appropriate approach. As we have seen, the time constant for strange hadron production is about $10^{-21} \mathrm{~s}$ and hence it is fully consistent to ignore all (even infinitely many) states of high mass which will not have the slightest chance of being excited in collisions of individual hadrons during $10^{-22} \mathrm{~s}$ duration of the high energy nuclear collision, except in the case of a transitory formation of quark-gluon plasma which we do not consider in this section.

\subsection{Rate equations for the production of strange particles}

We now proceed to develop the evolution equations for the densities of particles included in the time evolution calculations. Note that only the time evolution of the densities and total particle numbers will be considered and the evolution of momentum distributions has not been investigated.

The reason behind this neglect of a treatment of the momentum space evolution is, of course, the belief that the rates for approach to a thermal phase space distribution are at least an order of magnitude larger than those governing chemical equilibration. This is certainly correct for strangeness production, as the results discussed below indicate. (For reactions among nonstrange hadrons such a division into chemical and thermal equilibrium may not be possible, since excited hadronic states, e.g. the $\rho$ or the $\Delta$, play a double role as chemical species and as resonances in the interaction $S$-matrix.)

The momentum integrated time evolution equations take the general form (see appendix $A$ for explicit form) 


$$
\frac{\mathrm{d}}{\mathrm{d} t} \rho_{i}(t)=\sum_{\alpha}\left\{\left\langle\sigma_{\alpha} v\right\rangle \prod_{k_{\alpha}} \rho_{k}(t)\right\}-\sum_{\beta}\left(\left\langle\sigma_{\beta} v\right\rangle \rho_{i}(t) \prod_{k_{\beta}} \rho_{k}(t)\right)
$$

where the index $i, k$ denotes the constituent particles $\pi, \mathrm{N}, \mathrm{Y}, \Xi$ etc., and their antiparticles, we do not distinguish different isospin states, and $\beta$ sums over all reactions producing and resp. depleting the population of the constituent $i$. Index $k_{\alpha}$ denotes the particles (typically two) which participate in the production reaction $\alpha$, while $k_{\beta}$ counts the reaction components (excluding $i$ ) in the depleting reaction.

The quantity $\left\langle\sigma_{\alpha} v\right\rangle$ is the (thermal) averaged cross section for an initial channel to lead to a specific final channel including particle $i$. For two-body initial states, denoting by $\mathrm{a}, \mathrm{b}$ the two particles, we have:

$$
\left\langle\sigma_{\mathrm{ab}}^{n} v_{\mathrm{ab}}\right\rangle=\frac{\int \mathrm{d}^{3} P_{\mathrm{a}} \mathrm{d}^{3} P_{\mathrm{b}} f_{\mathrm{a}}\left(P_{\mathrm{a}}\right) f_{\mathrm{b}}\left(P_{\mathrm{b}}\right) \sigma_{\mathrm{ab}}^{n} v_{\mathrm{ab}}}{\int \mathrm{d}^{3} P_{\mathrm{a}} \mathrm{d}^{3} P_{\mathrm{b}} f_{\mathrm{a}}\left(P_{\mathrm{a}}\right) f_{\mathrm{b}}\left(P_{\mathrm{b}}\right)}
$$

where $\sigma_{\mathrm{ab}}^{n}$ is the cross section for the $\mathrm{a}+\mathrm{b} \rightarrow n$-bodies, $v_{\mathrm{ab}}$ the relative velocity of the incoming particles and $f_{\mathrm{a}}\left(P_{\mathrm{a}}\right), f_{\mathrm{b}}\left(P_{\mathrm{b}}\right)$ for their momentum distributions, which are explicitly renormalized above such that

$$
\int \mathrm{d}^{3} P_{i} f_{i}\left(P_{i}\right)=1
$$

For almost all particles in the hadronic gas it is sufficient to use the Boltzmann momentum distribution function, but for pions further improvement is required.

The value of $\langle\sigma v\rangle$ at a given temperature depends strongly on the threshold energy required to make the transition from the initial to the final state. In particular, $\sigma$ is zero unless the energy of the incoming channel in the centre-of-mass system is greater than the sum of the rest masses of the particles in the final channel. This is made explicit if we write $\sigma$ in terms of $T$-matrix elements [KB73]:

$$
\sigma_{\mathrm{ab}}^{n}=F^{-1} \int \prod^{n} \mathrm{~d}^{4} P_{i} \delta\left(P_{i}^{2}-m_{i}^{2}\right) \Theta\left(P_{i}^{0}\right) \delta^{4}\left(P_{\mathrm{a}}+P_{\mathrm{b}}-\sum_{i}^{n} P_{i}\right)|\langle\mathrm{f}|T| i\rangle|^{2}
$$

where

$$
F=2\left\{\left[s-\left(m_{\mathrm{a}}+m_{\mathrm{b}}\right)^{2}\right]\left[s-\left(m_{\mathrm{a}}-m_{\mathrm{b}}\right)^{2}\right]\right\}^{1 / 2}(2 \pi)^{3 n-4}
$$

and, as usual, $s^{1 / 2}$ is here the total energy in the c.m. system (and not strangeness or entropy).

Instead of calculating the transition probabilities

$$
|M|^{2}:=|\langle\mathrm{f}|T| i\rangle|^{2}
$$

from a suitable reaction model - which is not explicitly feasible at present - measured cross sections $\sigma_{\mathrm{ab}}^{n}(s)$ are used in a functional parameterisation of the data in terms of the centre-of-mass energy $\sqrt{s}$. With some algebra and in the Boltzmann approximation for the distribution functions it is then possible to write $(\sigma v)$, eq. $(5.5)$ in the form 


$$
\left\langle\sigma_{\mathrm{ab}}^{n} v_{\mathrm{ab}}\right\rangle=\frac{\beta}{8} \frac{\int_{\sqrt{s_{0}}}^{\infty} \mathrm{d} \sqrt{s} \sigma(\sqrt{s})\left[s-\left(m_{\mathrm{a}}+m_{\mathrm{b}}\right)^{2}\right]\left[s-\left(m_{\mathrm{a}}-m_{\mathrm{b}}\right)^{2}\right] K_{1}(\beta \sqrt{s})}{m_{\mathrm{a}}^{2} \overline{m_{\mathrm{b}}^{2}} \overline{K_{2}\left(\beta m_{\mathrm{a}}\right) K_{2}\left(\beta m_{\mathrm{b}}\right)}}
$$

where $\sqrt{s_{0}}$ is the threshold denoting the lowest energy necessary to allow for the reaction in the centre-of-mass system of the two colliding particles under consideration. One should note that the average (5.7) is weighted with the factor $K_{1}\left(\beta \sqrt{s_{0}}\right)$ which for many reactions is a small number.

Equation (5.7) is derived as follows: for the denominator of (5.5a) one simply finds, using standard expressions for Boltzmann momentum distributions:

$$
\begin{aligned}
& f_{i}(P)=\exp \left(-\beta \sqrt{P_{i}^{2}+m_{i}^{2}}\right) \\
& \int \mathrm{d}^{3} P_{i} f_{i}\left(P_{i}\right)=4 \pi m_{i}^{2} \beta^{-1} K_{2}\left(\beta m_{i}\right)
\end{aligned}
$$

with $\beta=T^{-1}$ being the inverse temperature. The nominator of eq. (5.5a) is now recast into the covariant form:

$$
\begin{aligned}
\int \frac{\mathrm{d}^{3} P_{\mathrm{a}}}{2 E_{\mathrm{a}}} \frac{\mathrm{d}^{3} P_{\mathrm{b}}}{2 E_{\mathrm{b}}} f_{\mathrm{a}}\left(P_{\mathrm{a}}\right) f_{\mathrm{b}}\left(P_{\mathrm{b}}\right) \sigma(s) v_{\mathrm{ab}} 2 E_{\mathrm{a}} 2 E_{\mathrm{b}} \\
=\int \mathrm{d}^{4} P_{\mathrm{a}} \mathrm{d}^{4} P_{\mathrm{b}} f_{\mathrm{a}}\left(P_{\mathrm{a}}\right) f_{\mathrm{b}}\left(P_{\mathrm{b}}\right) \delta_{0}\left(P_{\mathrm{a}}^{2}-m_{\mathrm{a}}^{2}\right) \delta_{0}\left(P_{\mathrm{b}}^{2}-m_{\mathrm{b}}^{2}\right) \sigma(s) \lambda_{\mathrm{in}}^{1 / 2}(s),
\end{aligned}
$$

where we have rewritten the expressions to be manifestly covariant

$$
\begin{aligned}
& \int \frac{\mathrm{d}^{3} P_{i}}{2 E_{i}}=\int \mathrm{d}^{4} P_{i} \delta_{0}\left(P_{i}^{2}-m_{i}^{2}\right), \\
& v_{\mathrm{ab}} 2 E_{\mathrm{a}} 2 E_{\mathrm{b}}=\lambda_{\mathrm{in}}^{1 / 2}(s)=\left\{\left[s-\left(m_{\mathrm{a}}+m_{\mathrm{b}}\right)^{2}\right]\left[s-\left(m_{\mathrm{a}}-m_{\mathrm{b}}\right)^{2}\right]\right\}^{1 / 2} .
\end{aligned}
$$

The covariantly written Boltzmann distributions $f_{i}\left(P_{i}\right)=\exp \left(-\beta \cdot P_{i}\right)$ (in c.m. form $\beta_{\mu}=(1 / T, 0)$ ) are additive in the momenta

$$
f_{\mathrm{a}}\left(P_{\mathrm{a}}\right) f_{\mathrm{b}}\left(P_{\mathrm{b}}\right)=\int \mathrm{d}^{4} P \mathrm{e}^{-\beta P} \delta^{4}\left(P-P_{\mathrm{a}}-P_{\mathrm{b}}\right)
$$

and $P$ is the total momentum of the incoming particles. Using $\int \mathrm{d} s \delta\left(P^{2}-s\right)=1$ we can thus write:

$$
\int \mathrm{d}^{3} P_{\mathrm{a}} \mathrm{d}^{2} P_{\mathrm{b}} f_{\mathrm{a}}\left(P_{\mathrm{a}}\right) f_{\mathrm{b}}\left(P_{\mathrm{b}}\right) \sigma(s) v_{\mathrm{ab}}=\int_{\mathrm{s}_{0}}^{\infty} \mathrm{d} s \sigma(s) \lambda_{\mathrm{in}}^{1 / 2}(s) \int \mathrm{d}^{4} P \mathrm{e}^{-\beta P} \delta\left(P^{2}-s\right) .
$$

The last integral is now nothing more than the relativistic invariant momentum phase space for two particles and equals 


$$
\int \mathrm{d}^{4} P_{\mathrm{a}} \mathrm{d}^{4} P_{\mathrm{b}} \delta^{4}\left(P-P_{\mathrm{a}}-P_{\mathrm{b}}\right) \delta_{0}\left(P_{\mathrm{a}}^{2}-m_{\mathrm{a}}^{2}\right) \delta_{0}\left(P_{\mathrm{b}}^{2}-m_{\mathrm{b}}^{2}\right)=(\pi / 2) s^{-1} \lambda_{\mathrm{in}}^{1 / 2}(s)
$$

Recall further:

$$
\int \mathrm{d}^{4} P \mathrm{e}^{-\beta P} \delta\left(P^{2}-s\right)=\frac{2 \pi}{\beta} \sqrt{s} K_{1}(\beta \sqrt{s}) .
$$

Inserting eqs. (5.12) and (5.13) in (5.11) and using eq. (5.8) leads, when inserted into eq. (5.5) straightforwardly to expression (5.7).

Turning back now to the explicit evaluation of the thermal averages $\langle\sigma v\rangle$ we note that experimental information is available for the following reactions
(a) $\pi+\mathrm{N} \rightarrow \mathrm{K}+\mathrm{Y} \quad$ 'strangeness production'
(only charged pions)
(b) $\overline{\mathrm{K}}+\mathrm{N} \rightarrow \mathrm{Y}+\pi \quad$ 'strangeness exchange'
(only charged kaons)
(c) $\mathrm{p}+\overline{\mathrm{p}} \rightarrow 5 \pi \quad$ 'annihilation'.

More details about the reaction channels used and cross section parameterization are given in appendix B. Fortunately, there is for each class of above-mentioned reactions at least one experimental cross section which can be used to calculate the corresponding thermal averages.

In the case of particular reactions for which no experimental information is available, one can proceed by assuming that the $T$-matrix elements contained in the expressions for the cross sections are only weakly dependent on the transferred momentum in the considered energy range. This assumption seems to be valid for low centre-of-mass energies in hadron-hadron reactions [KB73].

We proceed to exploit these assumptions. Taking the nearly constant matrix element out of the integral in eq. (5.6) we find for the numerator of eq. (5.6a):

$$
\begin{aligned}
I_{n} & \simeq|M(\mathrm{a}+\mathrm{b} \rightarrow n)|^{2} \int \prod_{i}^{n} \mathrm{~d}^{4} P_{i} \delta_{0}\left(P_{i}^{2}-m_{i}^{2}\right) \delta\left(P-\sum_{i} P_{i}\right) \\
& :=|M(\mathrm{a}+\mathrm{b} \rightarrow n)|^{2} \operatorname{IMS} n\left(P^{2}, m_{i}\right)
\end{aligned}
$$

which permits us to write for the integral in the numerator of eq. (5.5)

$$
|M|^{2} \int_{s_{0}}^{\infty} \mathrm{d} s \operatorname{IMS} 2\left(\text { in) IMSn(out) } \int \mathrm{d}^{4} P \mathrm{e}^{-\beta P} \delta\left(P^{2}-s\right)\right.
$$

The last integral above being given in eq. (5.13), we obtain: 


$$
\left\langle\sigma_{\mathrm{ab}}^{n} v_{\mathrm{ab}}\right\rangle=\frac{|M(\mathrm{a}+\mathrm{b} \rightarrow n)|^{2}}{(2 \pi)^{3 n-3} 4} \frac{\int_{\sqrt{s_{0}}}^{\infty} \mathrm{d} \sqrt{s} s \operatorname{IMS} 2\left(\text { in) } \operatorname{IMS} n \text { (out) } K_{1}(\beta \sqrt{s})\right.}{m_{\mathrm{a}}^{2} K_{2}\left(\beta m_{\mathrm{a}}\right) m_{\mathrm{b}}^{2} K_{2}\left(\beta m_{\mathrm{b}}\right) \beta^{-1}}
$$

with $|M|^{2}=|\langle\mathrm{f}|T| i\rangle|^{2}$ and IMS2(in), IMSn(out) being the two- and $n$-particle invariant phase space volumes of the in- and out-going particles as defined by eq. (5.14). In the two-particle case

$$
\operatorname{IMS} 2=(\pi / 2) s^{-1}\left\{\left[s-\left(m_{1}+m_{2}\right)^{2}\right]\left[s-\left(m_{1}-m_{2}\right)^{2}\right]\right\}^{1 / 2} .
$$

In the relativistic limit, all masses can be neglected and

$$
\operatorname{IMS} n \cong\left(\frac{\pi}{2}\right)^{n-1} \frac{1}{(n-1) !(n-2) !} s^{n-2} .
$$

The relativistic approximation eq. (5.16b) introduces in our studies a tolerable error $(\sim 20 \%)$ in the framework of accuracy desired here.

Since the same constant matrix element for each underlying subprocess is used, only the phase space dependence of the different reactions remains. Thus, for example, the strangeness production cross section in its thermal average, experimentally given $\left\langle\sigma_{\pi N}^{\bar{K} Y} v_{\pi N}\right\rangle$, may be used to compute

$$
\left\langle\sigma_{\pi \mathrm{Y}}^{\mathrm{K} \Xi} v_{\pi \mathrm{Y}}\right\rangle=\left\langle\sigma_{\pi \mathrm{N}}^{\mathrm{KY}} v_{\pi \mathrm{N}}\right\rangle P_{1} ; \quad P_{1}=\left\langle\sigma_{\pi \mathrm{Y}}^{\mathrm{K} \Xi} v_{\pi \mathrm{Y}}\right\rangle /\left\langle\sigma_{\pi \mathrm{N}}^{\mathrm{KY}} v_{\pi \mathrm{N}}\right\rangle
$$

where $P_{1}$ now, per assumption of constant $|M|^{2}$, only depends on $T$ through its dependence on particle phase space. Similarly,

$$
\begin{array}{ll}
\left\langle\sigma_{\pi \Xi}^{\mathrm{K} \Omega} v_{\pi \Xi}\right\rangle=\left\langle\sigma_{\pi \mathrm{N}}^{\mathrm{KY}} v_{\pi \mathrm{N}}\right\rangle P_{2} ; & P_{2}=\left\langle\sigma_{\pi \Xi}^{\mathrm{K} \Omega} v_{\Xi \pi}\right\rangle /\left\langle\sigma_{\pi \mathrm{N}}^{\mathrm{KY}} v_{\pi \mathrm{N}}\right\rangle \\
\left\langle\sigma_{\pi \pi}^{\mathrm{K} \overline{\mathrm{K}}} v_{\pi \pi}\right\rangle=\left\langle\sigma_{\pi \mathrm{N}}^{\mathrm{KY}} v_{\pi \mathrm{N}}\right\rangle P_{3} ; & P_{3}=\left\langle\sigma_{\pi \pi}^{\mathrm{K} \overline{\mathrm{K}}} v_{\pi \pi}\right\rangle /\left\langle\sigma_{\pi \mathrm{N}}^{\mathrm{KY}} v_{\pi \mathrm{N}}\right\rangle
\end{array}
$$

where all $P_{i}$ depend only on the phase space.

In an analogous manner one obtains the mean thermal cross sections falling under the categories 'strangeness exchange' and 'annihilation'. The various exchange cross sections are normalized by

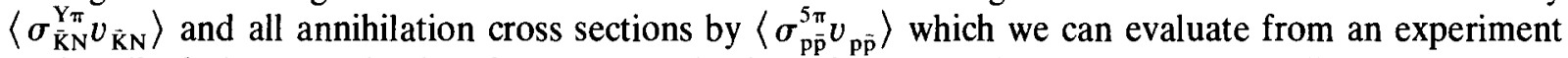
as described above, and using the parameterization of cross sections given in appendix $\mathrm{B}$.

The matrix element $|M|^{2}$ for reactions where particles and antiparticles are interchanged are the same, and the mean thermal cross section of the reverse reactions are given by those of the forward reactions times the constant which weights the equilibrium densities of the incoming articles by the outgoing ones. The equilibrium densities of various hadronic gas constituents are discussed in the following section 6 .

We conclude that in this framework there are three typical reaction cross sections, viz. 'strangeness production', 'strangeness exchange' and 'baryon annihilation'. In fig. 5.2 we present the thermally averaged strangeness formation and exchange cross sections obtained within the above outlined framework. We note that strangeness exchange reactions leading to multiple strange clusters are substantial, 1-3 mb at all temperatures. Strangeness formation reactions are dominated by the 


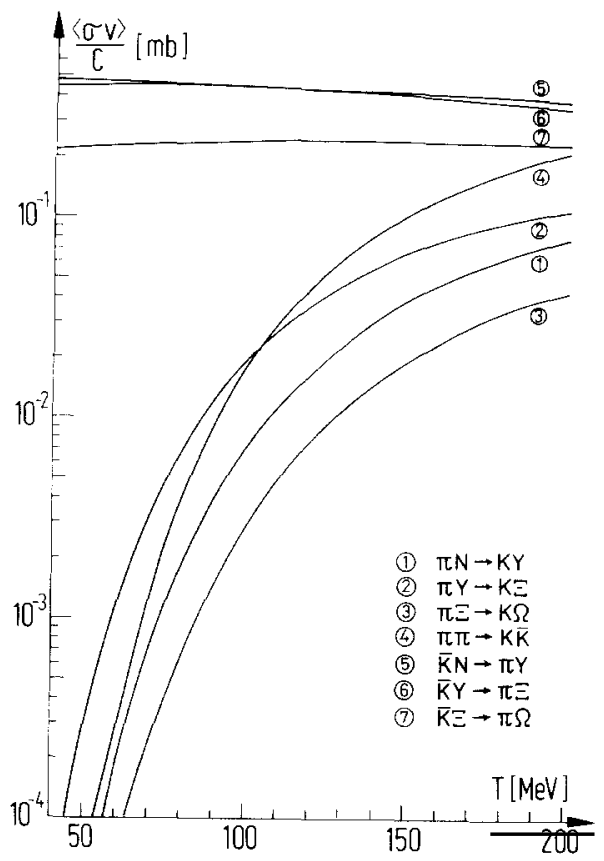

Fig. 5.2. Thermally averaged strangeness formation and exchange cross sections $\langle\sigma v\rangle / c$, based on the assumption of universal invariant matrix elements.

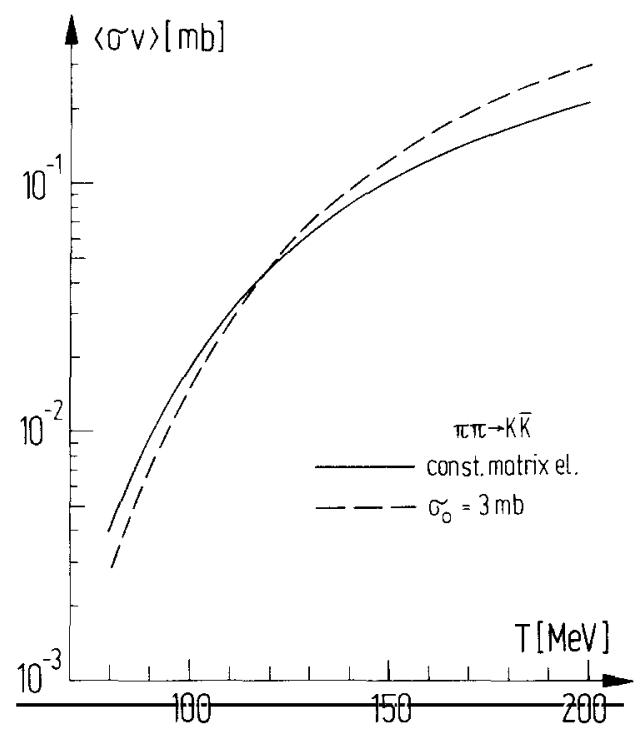

Fig. 5.3. Comparison of the predicted thermally averaged cross sections for the reaction $\pi \pi \rightarrow \mathrm{K} \overline{\mathrm{K}}$, based on the universal matrix element assumption (solid line) and on the assumption of constant cross section above threshold (dashed line).

$\pi \pi \rightarrow \mathrm{KK}$ reaction, which at $T=200 \mathrm{MeV}$ reaches the strength $0.2 \mathrm{mb}$. This behaviour confirms the previously stated assertion that relative chemical equilibrium involving baryons is much easier to achieve than the absolute saturation of the strange particle phase space.

We note that the cross section of the reaction $\pi \pi \rightarrow K \bar{K}$, which is an important channel for strangeness production in the thermally equilibrated hadronic gas, cannot be measured directly. It can, however, be derived from the reactions $\mathrm{pp} \rightarrow \mathrm{pp} \pi \pi, \mathrm{ppK \overline {K }}$ with the help of dispersion relations. Protopopescu et al. [Pr73] have found in this way that the cross section $\sigma(\pi \pi \rightarrow K \bar{K})$ is approximately constant above threshold with a value of $\sigma_{0}=3 \pm 1 \mathrm{mb}$. In order to check the approximate validity of our assumption we have compared in fig. 5.3 the results obtained for the thermally averaged cross section on the basis of the two different model assumptions: the solid line shows the prediction of the universal matrix element model (cf. fig. 5.2), while the dashed line shows the expected averaged cross section found by assuming constant cross section threshold. That the two results agree within the experimental bounds is reassuring.

The calculated average cross sections enter the nonlinear master equations, cf. appendix A, that describe the evolution of the chemical composition of the hot hadronic matter phase. Before these equations can be solved, it is necessary to specify the initial values of all the densities at a given time $t_{0}$. If one is interested in deciding whether multistrange particle abundances tell us whether a quark-gluon plasma phase has been temporarily produced in a nuclear collision, one must compare scenarios in which particle abundances are calculated as if the reaction were to proceed either entirely through hadronic matter or with the intermediate presence of a deconfined quark-gluon phase. In the first case, one may set the densities of strange particles at $t_{0}$ all equal to zero, neglecting direct strangeness production in the first moments of the collision before thermal equilibrium is reached. In the other case, 
strangeness is first produced in the quark-gluon plasma and then - in its break up - the strange quarks and antiquarks are built into hadrons. The hadronic reaction then starts with the strangeness abundance that has been built up in the quark-gluon plasma, and possibly modified in the phase transition back to hadronic matter. Predictions, therefore, require a specific model of the hadronization process, which we described in the previous section 4 .

\subsection{Approach to equilibrium of strangeness abundance in hot hadronic matter}

We shall now discuss the solution of the rate equations for the abundances of strange particles in a thermally, and isospin- but not strangeness, equilibrated hadronic gas. Let the production of strangeness commence at a time $t_{0}=1 \times 10^{-24} \mathrm{sec}$, since after that time the pion- and nucleon densities should have reached approximately their equilibrium values [Me82]. For the antinucleon density we use the chemical equilibrium value, being aware of the fact that the assumption of baryochemical equilibrium for antinucleons is overestimating the possible abundance. This leads to an overestimate of the abundance of strange antibaryons generated by hadronic interactions in the absence of quark-gluon plasma. A reduced initial abundance of antinucleons would cause a still longer equilibration time of strange antibaryons.

The time evolution of the hadronic gas system described above is fixed once the temperature $T$ and baryon chemical potential $\mu_{\mathrm{b}}$ are prescribed. For simplicity, we assume for the purpose of computing the characteristic times of equilibration and time evolution of particle abundances that $T$ and $\mu_{\mathrm{b}}$ are constant over space-time.

Let us begin the discussion with the study of the time evolution of the total strangeness abundance $\rho_{\mathrm{s}}$ which is calculated according to the quark content:

$$
\rho_{\mathrm{s}}=\rho_{\overline{\mathrm{K}}}+\rho_{\overline{\mathrm{K}}^{*}}+\rho_{\mathrm{Y}}+2 \rho_{\Xi}+3 \rho_{\Omega}
$$

where, as before, $\rho_{\mathrm{Y}}=\rho_{\Lambda}+\rho_{\mathrm{\Sigma}}$. Due to exact strangeness conservation, $\rho_{\mathrm{s}}$ is equal to the total antistrangeness density $\rho_{\bar{s}}$. Similar equations define light quark density or baryon number density.

The results of the calculation are shown in fig. 5.4 for $T=160 \mathrm{MeV}$ and two values of the baryon chemical potential, $\mu_{\mathrm{b}}=0$ and $\mu_{\mathrm{b}}=450 \mathrm{MeV}$. When looking at both curves, we see that after a typical break-up time of the hadronic fireball of about $5 \times 10^{-23} \mathrm{~s}$, the strangeness abundance predicted for the hadronic phase is still about a factor of three smaller than its equilibrium value. Individual particles with higher strangeness content such as $\bar{\Omega}$ (see below) will be still more distant from equilibrium distribution. One should be aware that complex circumstances of nuclear collisions in which no plasma was formed may further reduce strangeness abundance and that we have made rather optimistic assumptions about the equilibration time for the nonstrange antibaryons.

We next turn to the discussion of the multistrange baryons and antibaryons. We find that the density of anti-omegas as well as omegas is in HG a factor of about $10^{3}$ lower than their equilibrium values for a break-up time between $10^{-23}$ and $10^{-22} \mathrm{~s}$ (see fig. 5.5). This is true for baryon-less as well as for baryon-rich hadronic gas. Anticascades $(\bar{\Xi})$ are suppressed by a factor of about $10^{2}$ with respect to their equilibrium values. We emphasize again that in more realistic calculations with expansion and cooling of the hadronic fireball taken into account, and with a lower initial density of antinucleons than the equilibrium values taken here, one would end up with an even lower density of strange antibaryons after a given time $t$. Hence results presented in fig. 5.5 must be strictly considered as upper limits for plasma-less nuclear collisions. 


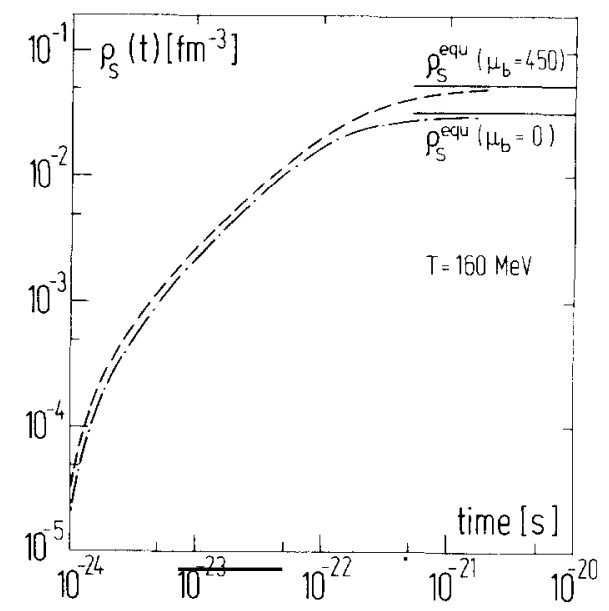
Fig. 5.4. Time evolution of strangeness density in hot hadronic matter at fixed temperature $T=160 \mathrm{MeV}$ and baryochemical potentials $\mu_{\mathrm{b}}=0$ and
$\mu_{\mathrm{b}}=450 \mathrm{MeV}$.

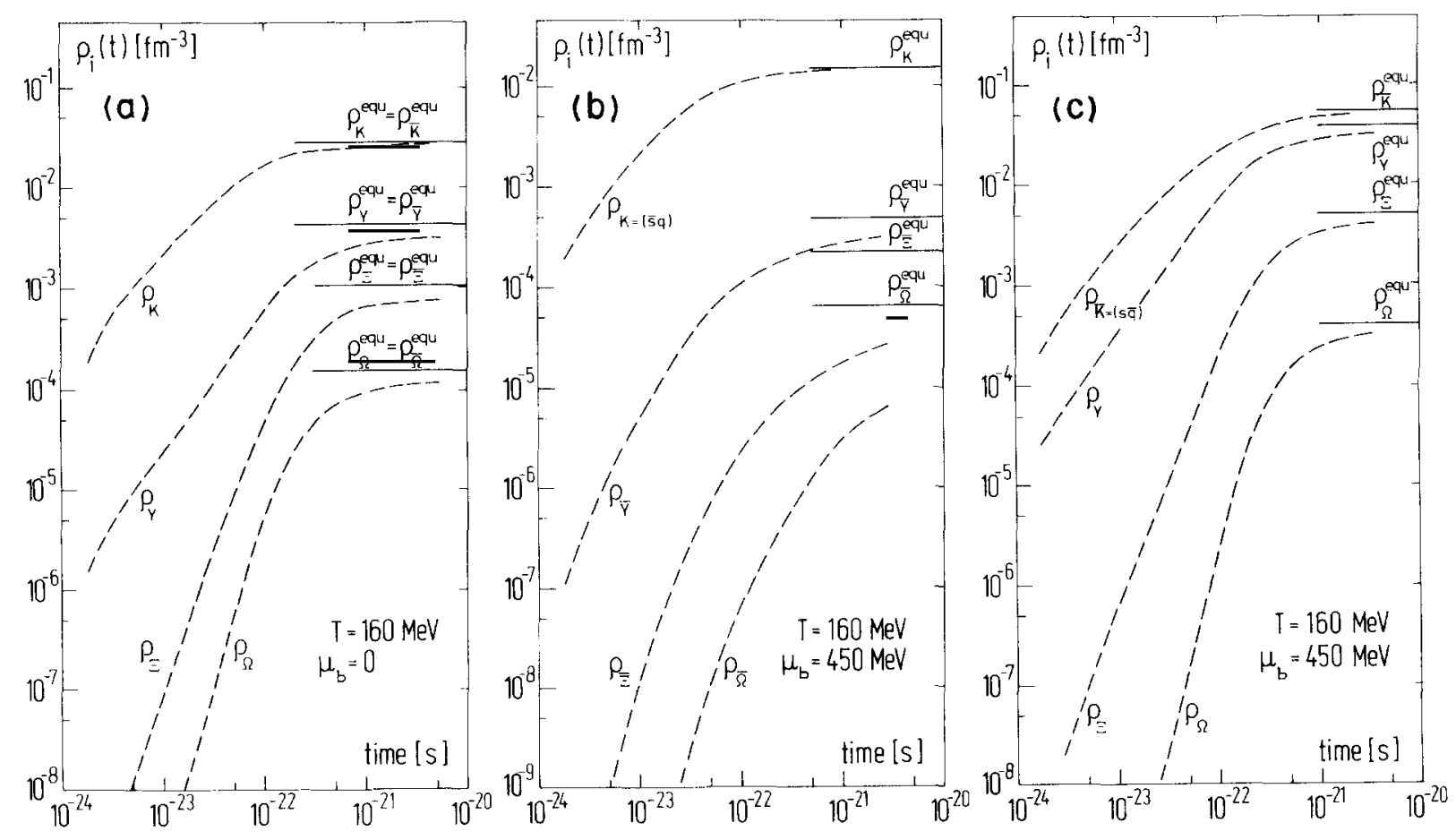

Fig. 5.5. Approach to equilibrium of various strange particle densities in hot hadronic matter at fixed temperature $T=160$ and $\mu_{\mathrm{b}}=0$ (a) and $\mu_{\mathrm{b}}=450 \mathrm{MeV}$ (b) and (c). In (a) at $\mu_{\mathrm{b}}=0$. Strange and antistrange particles have the same abundance. At finite baryon density ( $\mu_{\mathrm{b}}=450 \mathrm{MeV}$ ) $\bar{s}$-hadrons are shown in (b) while s-hadrons are shown in (c). 
For this reason, strange antibaryons appear as the most promising signal for the temporary existence of a deconfined quark-gluon phase during the course of the collision. As we have already seen in section 4 , the quark fragmentation-recombination model of quark gluon plasma leads to a quite substantial abundance of these rare hadronic states.

\section{Strangeness abundance in hadronic gas in chemical equilibrium}

We now consider abundances of strange particles in the chemically equilibrated hadronic gas phase. Although we have just shown that in the hadronic gas phase such a state is probably not attainable, this exercise is not entirely academic as various pre-equilibrium reactions on quark-gluon plasma seeds may produce the required strange particle abundance should precritical conditions for the formation of quark-gluon plasma be achieved. The $\mathrm{HG}$ is a very complicated state of hadronic matter due to its strong particle interactions with the presence of numerous hadronic resonances. But it turns out that just the postulate of the resonance-dominance of hadron-hadron interactions [Ha65] allows a vast simplification of the theoretical treatment. When regarding the hadronic gas phase as a mixture of various noninteracting hadronic resonance gases, all information about the interaction is hidden in the mass spectrum $\tau\left(m^{2}, b\right)$ which describes the number of hadrons of baryon number $b$ in a mass interval $\mathrm{d} m^{2}$.

In the grand canonical description one then finds that the nonstrange hadrons influence the strange ones by providing a background value of the chemical potentials, and in particular the baryon number chemical potential $\mu_{\mathrm{b}}$. This is best seen considering that the total partition function is additive as long as the various gas fractions interact only via particle resonances. We then have

$$
\ln Z=\ln Z^{\text {nonstrange }}+\ln Z^{\text {strange }} .
$$

In order to understand strange particle abundances, it is sufficient to consider $\ln Z^{\text {strange }}$ at given $\mu_{\mathrm{b}}$. However, the relationship between $\mu_{\mathrm{b}}$ and the baryon density $\rho_{\mathrm{b}}$ is controlled almost completely by the nonstrange particles and hence can not be determined by the considerations presented here. However $\mu_{\mathrm{b}}$, like $T$, is a thermodynamical variable accessible to direct measurement (see subsection 6.3) while $\rho_{\mathrm{b}}$ is difficult to measure directly.

\subsection{Grand canonical treatment}

Relatively low density of strange particles permits us to consider the grand canonical partition function in the Boltzmann approximation:

$$
\ln Z^{\mathrm{s}}\left(V, T ; \lambda_{i}\right)=\sum_{j} Z_{j} \prod_{\substack{i=\mathrm{b}, \mathbf{s}, \mathrm{Q}}}\left(\lambda_{i}^{(j)}\right)^{n_{j i}}
$$

where the one-particle Boltzmann partition functions

$$
Z_{j}(V, T)=g_{j}\left(V T^{3} / 2 \pi^{2}\right)\left(m_{j} / T\right)^{2} K_{2}\left(m_{j} / T\right)
$$


of the particle species $j$ with the corresponding degeneracy factor $g$ and mass $m$ appear. The fugacities $\lambda_{i}$ as introduced in eq. (6.2) control the quantum number content of the particle species $j$. We have: $\lambda_{\mathrm{s}}$ which controls the strange quark content, $\lambda_{\mathrm{b}}$ which controls the baryon number content and occasionally we also consider a $\lambda_{\mathrm{Q}}$ associated with the electric charge. The power $n_{j i}$ is the number of charges ' $i$ ' in the hadron ' $j$ '. The upper index $(j)$ indicates that we are looking at the fugacity associated with the given hadron $j$.

It is useful to record the explicit form of eq. (6.2) when only single strange hadrons are considered and isospin breaking effects are ignored:

$$
\ln Z^{\mathrm{s}=1}\left(V, T: \lambda_{\mathrm{b}}, \lambda_{\mathrm{s}}\right)=Z_{\mathrm{K}}\left(\lambda_{\mathrm{s}} / \lambda_{\mathrm{q}}+\lambda_{\mathrm{q}} / \lambda_{\mathrm{s}}\right)+Z_{\mathrm{Y}}\left(\lambda_{\mathrm{s}} \lambda_{\mathrm{q}}^{2}+\lambda_{\mathrm{s}}^{-1} \lambda_{\mathrm{q}}^{-2}\right)
$$

where

$$
\lambda_{\mathrm{q}}^{3}=\lambda_{\mathrm{b}}
$$

according to the fact that three light quarks make up a baryon and

$$
Z_{\mathrm{Y}}=\left(V T^{3} / 2 \pi^{2}\right)\left(2 W\left(x_{\Lambda}\right)+3 W\left(x_{\Sigma}\right)\right) \text {. }
$$

Here it is convenient to write $x_{i}=M_{i} / T$ and use the function

$$
W(x)=x^{2} K_{2}(x) \underset{x \rightarrow 0}{\longrightarrow} 2 .
$$

Similarly

$$
Z_{\mathrm{K}}=\left(V T^{3} / 2 \pi^{2}\right) 2 W\left(x_{\mathrm{K}}\right)
$$

Using the partition function (6.2), we can calculate the mean strangeness by evaluating

$$
S=\left\langle n_{\mathrm{s}}-n_{\overline{\mathrm{s}}}\right\rangle=\lambda_{\mathrm{s}} \frac{\partial}{\partial \lambda_{\mathrm{s}}} \ln Z\left(V, T, \lambda_{\mathrm{s}}, \ldots\right)
$$

which is the difference between strange and antistrange components. This expression must be equal to zero since strangeness is a conserved quantum number with respect to the strong interactions. This introduces an important constraint, e.g. it fixes $\lambda_{\mathrm{s}}$ in terms of $\lambda_{\mathrm{b}}$ (and $\lambda_{\mathrm{Q}}$ when considered). Let us ignore, in the first instance the multistrange particles. Then inserting eq. (6.4) in eq. (6.9) we find

$$
\gamma:=\lambda_{\mathrm{s}} / \lambda_{\mathrm{q}}=\left[\frac{Z_{\mathrm{K}}+\lambda_{\mathrm{b}}^{-1} Z_{\mathrm{Y}}}{Z_{\mathrm{K}}+\lambda_{\mathrm{b}} Z_{\mathrm{Y}}}\right]^{1 / 2} .
$$

We record a strong dependence of $\gamma$ on the baryon number. Recall that

$$
\lambda_{\mathrm{b}}=\exp \left(\mu_{\mathrm{b}} / T\right)
$$

and hence the term with $\lambda_{\mathrm{b}}^{-1}$ will tend to zero as $\mu_{\mathrm{b}}$ gets larger and the term with $\lambda_{\mathrm{b}}$ will dominate the 
expression for $\gamma$. As a consequence the particles with fugacity $\lambda_{s}$ (i.e. $\bar{q} s$, qqs) are slightly suppressed. Conversely, the abundance of particles which contain the strange antiquark ( $\mathrm{q} \overline{\mathrm{s}}, \overline{\mathrm{q}} \overline{\mathrm{q}} \overline{\mathrm{s}})$ will be favoured by the factor $\gamma^{-1}$ due to the presence of finite nuclear matter density. Further we note that $\mathrm{q}^{-} \mathrm{s}\left(\mathrm{K}^{+}, \mathrm{K}_{0}\right)$ kaons are the dominant carriers of $\bar{s}$-quarks, while qqs hyperon states are main carriers of s-quarks at finite baryon density. We further note that in eq. (6.10) $\lambda_{\mathrm{s}} \neq 1$, which is the case in quark-gluon plasma - it is for this reason that when quark-gluon plasma dissociates a nonequilibrium density of strange antibaryons emerges. Strange antibaryons are normally suppressed in equilibrated hadronic gas according to eq. $(6.10)$.

In order to obtain the mean equilibrium abundance of different strange particles, we introduce for each species its own dummy fugacity (which we subsequently will set equal to unity). Recall that the different strange particles need to reach relative chemical equilibrium for the calculation of ratio of abundances to be valid. Remember, however, that strange antibaryons will not follow this assumption while strange mesons and baryons, in general, approach the relative chemical equilibrium expediently.

We further observe that even assuming absolute chemical equilibrium in the gas phase we found in section 4 , in comparison, five times more strange hadrons from fragmentation-recombination of the plasma in comparable thermodynamic conditions. The baryon density in the hadronic phase is, however, lower if a first order phase transition is encountered. Due to this effect, the total equilibrium strangeness abundance would be nearly equal in both phases, as emphasized correctly by Redlich [Re85]. However, we note that the approach to equilibrium will be even further delayed in the thinner gas phase assumed by Redlich than it is otherwise, resulting in effectively still smaller expected strangeness abundance in the hadronic gas phase. No matter how one looks at it, all strangeness is always produced in QGP phase simply because the density of gluons is so extraordinarily enhanced there, due to their colour degeneracy factor 8 and their masslessness.

In fig. 6.1(a,b,c) we have shown three examples, namely, the ratios of antihyperons to hyperons. The explicit expressions for these ratios turn out to be very simple:

$$
\begin{aligned}
& \left\langle n_{\bar{\Lambda}}\right\rangle /\left\langle n_{\Lambda}\right\rangle=\lambda_{\mathrm{q}}^{-4} \lambda_{\mathrm{s}}^{-2}=\lambda_{\mathrm{b}}^{-2} \gamma^{-2} \\
& \left\langle n_{\bar{\Xi}}\right\rangle /\left\langle n_{\Xi}\right\rangle=\lambda_{\mathrm{q}}^{-2} \lambda_{\mathrm{s}}^{-4}=\lambda_{\mathrm{b}}^{-2} \gamma^{-4} \\
& \left\langle n_{\bar{\Omega}}\right\rangle /\left\langle n_{\Omega}\right\rangle=\lambda_{\mathrm{s}}^{-6}=\lambda_{\mathrm{b}}^{-2} \gamma^{-6} .
\end{aligned}
$$

Note in passing that the actual value of $\lambda_{\mathrm{s}}$ is slightly different from $\lambda_{\mathrm{s}}$ as defined in eq. (6.10) when multiply strange hadrons are also counted in the strangeness conservation relation eq. (6.9). We observe in fig. 6.1c that the enhancement of antistrangeness due to the factor $\gamma^{-6}$ is visible in the ratio $\bar{\Omega} / \Omega$ which approaches unity for large temperatures over the whole $\mu_{\mathrm{b}}$-region. Such a behaviour of the equilibrium abundances is not reflecting on the abundances developing in actual nuclear collision, since hadronic gas reactions cannot excite equilibrium density in $\bar{\Omega}$ as discussed at length in section 5 . The abundance of $\bar{\Omega}$ can be significant only if quark-gluon plasma has been formed.

In fig. 6.1a the expected suppression of $\widehat{\Lambda}$ at finite baryochemical potential because of the strangeness chemistry is recorded. This ratio exhibits both a strong temperature and $\mu_{\mathrm{b}}$ dependence. The remarkably small upper limit abundance of $\bar{\Lambda}$, i.e. $10^{-4} \Lambda$ of the $\Lambda$-abundance in hadronic gas phase, under conditions likely to be reached in an experiment at the end of the hadronization phase $\left(T \sim 120-180 \mathrm{MeV}, \mu_{\mathrm{b}} \sim(4-6) T\right)$ is characteristic of the nature of the hot hadronic matter phase. In section 4 we have obtained much higher estimates $\bar{\Lambda} / \Lambda$ ratio initially fed by the quark-gluon plasma into 

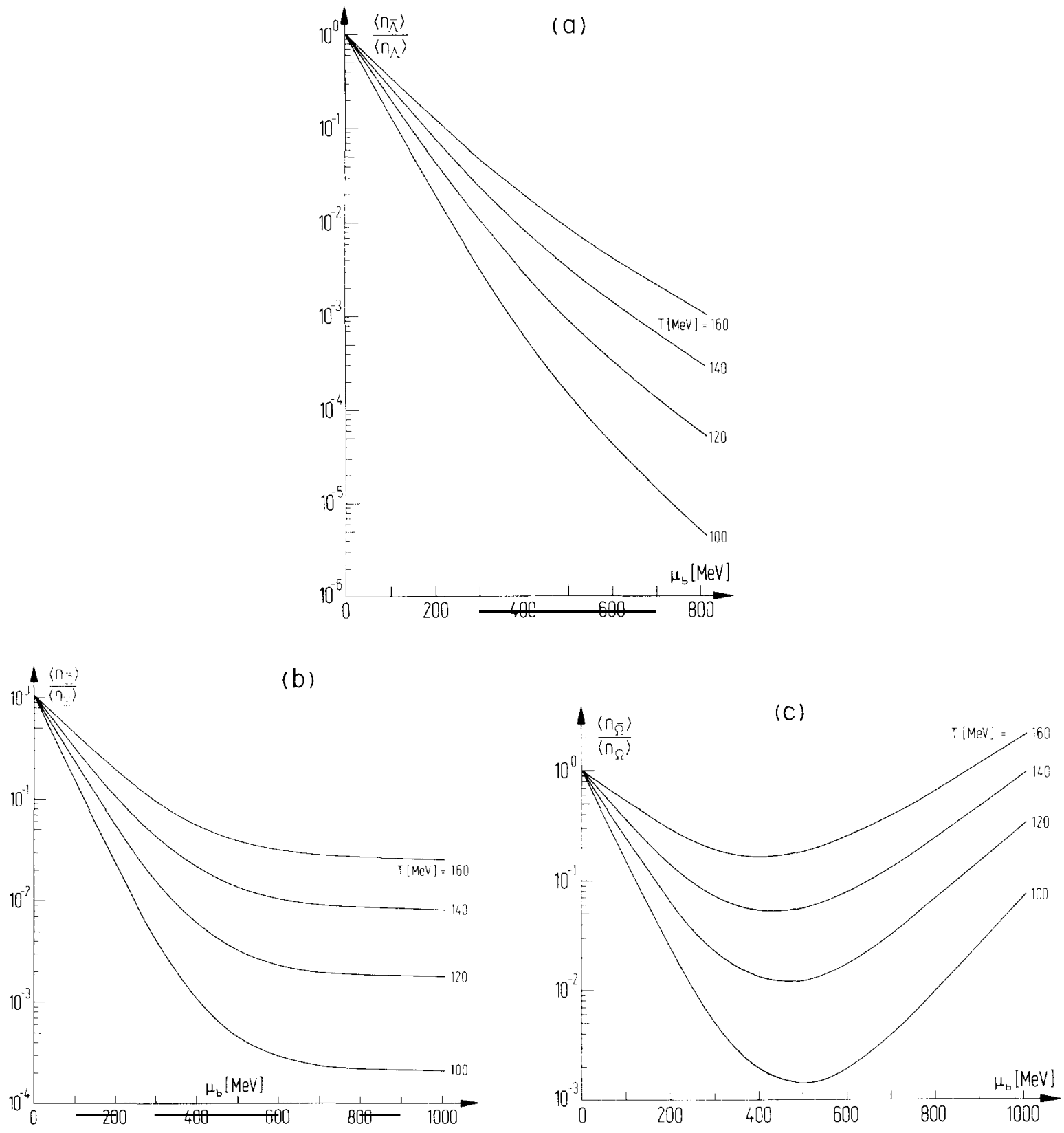

Fig. 6.1. Strange baryon/antibaryon particle ratios in hadronic gas equilibrium abundance ratio of $\bar{\Lambda} / \Lambda$ in dependence on baryochemical potential $\mu_{\mathrm{b}}$ at fixed temperature $T:$ (a) $\tilde{\Lambda} / \Lambda ;$ (b) $\Xi / \Xi ;$ (c) $\bar{\Omega} / \Omega$.

the hadronic phase. The actual final abundance of antihyperons expected from a transient quark-gluon plasma phase must be computed in an off-equilibrium approach by taking the initial values obtained in section 4 and using the methods of section 5, coupled with a dynamical model, to evolve the densities until essentially all particle-particle interactions disappear. Here presented equilibrium ratios are to be taken as upper limits in the instance that no quark-gluon plasma has been formed. 


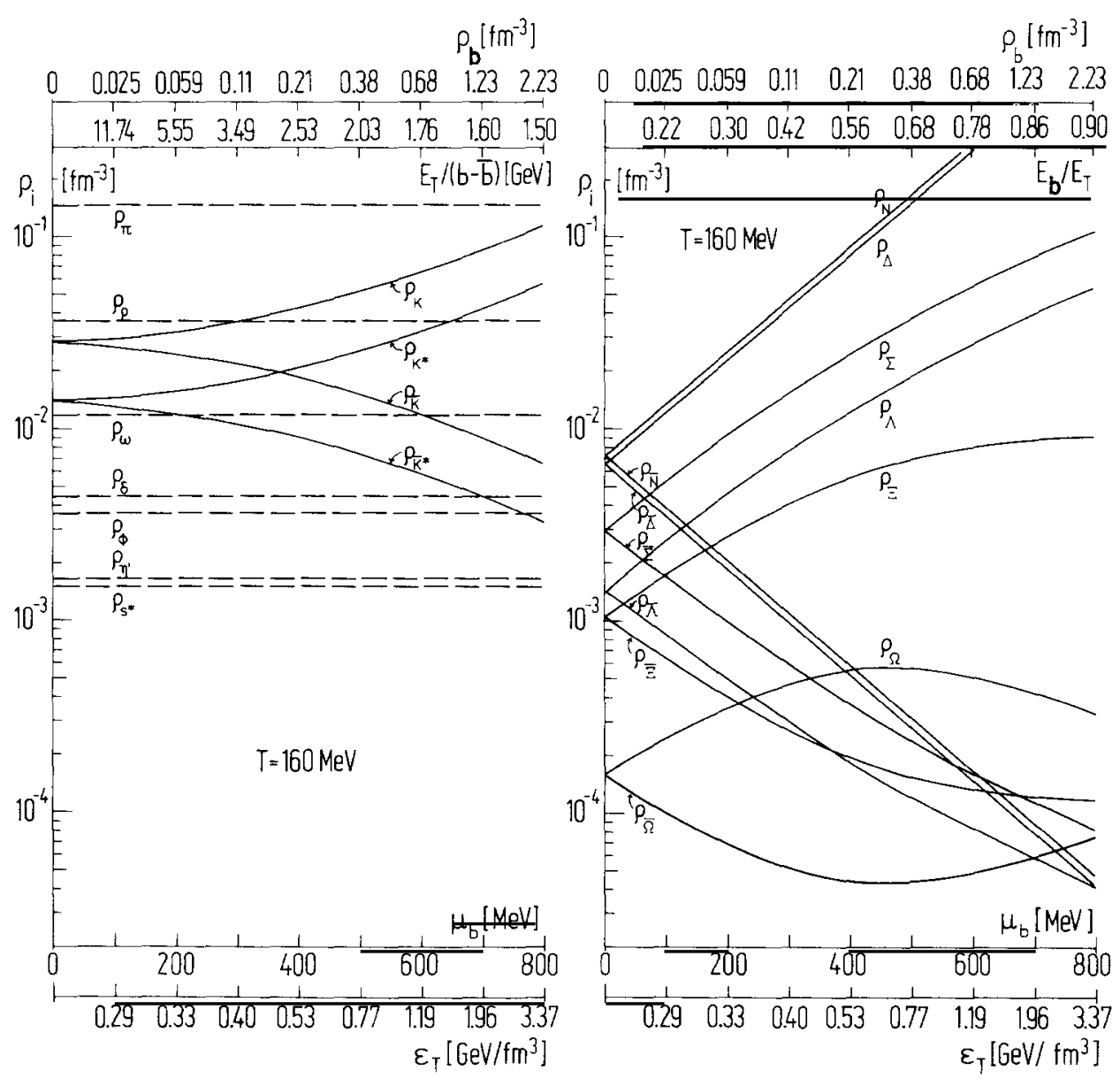

Fig. 6.2. Particle densities in equilibrated hadronic gas at $T=160 \mathrm{MeV}$, as a function of baryochemical potential $\mu_{\mathrm{b}}$.

Figure 6.2 describes in a most complete way the strange particle contents of the hadronic gas at $T=160 \mathrm{MeV}$ as a function of the baryochemical potential: The meson (left) and baryon-antibaryon densities (right hand side) are presented. We include (dashed) nonstrange mesons and also $\mathrm{N}$ and $\Delta$ baryons. The total energy density of the particles shown is displayed in parallel to the $\mu_{\mathrm{b}}$ axis at the bottom, while the baryon density is displayed horizontally on the top. We also show the energy per baryon (left) and the fraction of the energy in the baryons (right).

First we note that $T / \mu_{\mathrm{q}}=1$, i.e. $T / \mu_{\mathrm{b}}=\frac{1}{3} \mu_{\mathrm{b}} / T=3$, our favourite value in the foregoing discussion corresponds to an energy density $E_{T}=1 \mathrm{GeV} / \mathrm{fm}^{3}$, energy per baryon $\sigma_{T} / \mathrm{b}=E_{T} /(\mathrm{B}-\overline{\mathrm{B}})=1.9 \mathrm{GeV}$ and baryon density $\rho_{\mathrm{b}}-5 \rho_{0}$, the latter being completely dominated by $\rho_{\mathrm{N}}$ and $\rho_{\Delta}$. At this value of $\mu_{\mathrm{b}}$ we clearly see suppression of strange antibaryons against strange baryons. Recall this equilibrium result is not confirmed by our nonequilibrium and phase transition calculations. More details about the relation between energies and particle densities at $T=160 \mathrm{MeV}$ as a function of $\mu_{\mathrm{b}}$ is given in fig. 6.3. It cannot be emphasized often enough that all quantities assume values accessible to nuclear collisions of $50 \mathrm{GeV} / \operatorname{nucl}(\mathrm{Lab})$ when $\mu_{\mathrm{b}} \sim 450-600 \mathrm{MeV}$. 


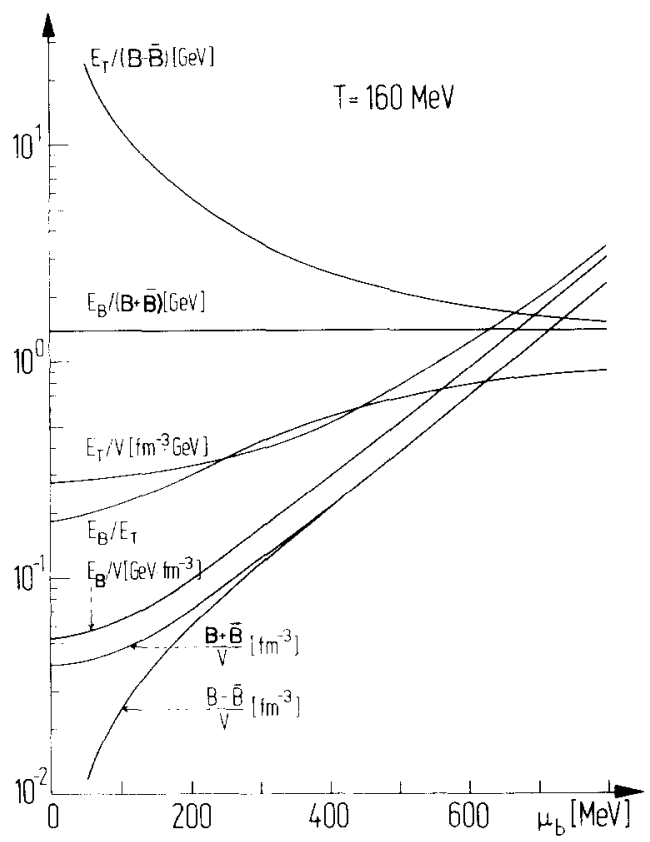

Fig. 6.3. Energy density $E_{T} / V$, energy per baryon $E_{T} /(\mathrm{B}-\overline{\mathrm{B}})$, baryon density $(\mathrm{B}-\overline{\mathrm{B}}) / V$ as a function of $\mu_{\mathrm{b}}$ for $T=160 \mathrm{MeV}$ equilibrated hadronic gas. Only particles shown in fig. 6.2 contribute to quantities displayed here.

\subsection{State of chemical equilibration in hadron-nucleus reactions}

We have, by now, largely settled the theoretical framework for analysing strange particle abundances in both possible phases of hadronic matter. We have shown that consideration of the dynamical approach of the abundance to the maximum available phase space density is an essential cornerstone of the study of both phases of hadronic matter. Our intention now is to show that hadron-nucleus collisions at high centre-of-mass energies show a degree of chemical equilibration among strange particles which cannot be achieved in a phase in which the process of chemical equilibration is based on reactions between individual hadrons only. While the intermediate state formed may not have the character of a plasma, it most certainly involves the subnuclear degrees of freedom.

Let us first consider certain $p-p$ and $p$-nucleus experiments where the data were analysed in the framework of a chemical equilibrium hadronic gas model [GKR84]. Since we want to describe different particle multiplicities, including the rapidity distribution of the baryon number, it is necessary to find for the baryochemical potential $\mu_{\mathrm{b}}$, controlling the baryon number, a dynamical distribution interpolating between the central and projectile rapidity regions. The linear relationship

$$
|x| m_{\mathrm{N}}=\mu_{\mathrm{b}}
$$

has been proposed by [GKR84] with $x$ being the usual Feynman variable, viz. the fraction of the maximal momentum possible a particle carries when detected. $m_{\mathrm{N}}=940 \mathrm{MeV}$ is the nucleon mass.

The physics underlying this approach is the hypothesis that the valence quarks of one nucleon penetrate the target rather freely without much interaction, while much of the energy and momentum in the gluon field remains in the central reaction region. This region, therefore, should exhibit smaller 
net baryon and charge number whereas in the maximum rapidity region we would expect to see the quantum numbers of the incoming particles, that is the baryon number is found mainly in the projectile (and target) fragmentation regions. A dynamical relation between $x$ and $\mu_{\mathrm{b}}$ fulfilling these requirements is given by eq. (6.13) - for $x= \pm 1$ (projectile/target region) we have $\mu_{\mathrm{b}} \rightarrow m_{\mathrm{N}}$ and for $x=0$ (central region) we have $\mu_{\mathrm{b}}=0$. Note that eq. (6.13) is not applying to nucleus-nucleus collisions at energies considered here, in which we expect that quark currents are stopped in the central region as well (see section 1).

The approach outlined in section 6.1 is applied incorporating, aside from baryon number $(b)$ and strangeness $(S)$ the conservation of the electric charge $(Q)$. Charge conservation is necessary in the light of experimental data when needing to distinguish up- and down-quarks: the proton, used as a projectile, has twice as high u-quark than d-quark abundance.

For the singly strange particles we have:

$$
\begin{aligned}
& \left\langle n_{\mathrm{K}^{+}}\right\rangle /\left\langle n_{\mathrm{K}^{-}}\right\rangle=\lambda_{\mathrm{Q}}^{2} \lambda_{\mathrm{s}}^{-2} \lambda_{\mathrm{q}}^{2} \\
& \left\langle n_{\bar{\Lambda}}+n_{\bar{\Sigma}^{0}}\right\rangle /\left\langle n_{\Lambda}+n_{\Sigma^{0}}\right\rangle=\lambda_{\mathrm{q}}^{-4} \lambda_{\mathrm{s}}^{-2} \\
& \left\langle n_{\Sigma^{\mp}}\right\rangle /\left\langle n_{\Sigma^{\ddagger}}\right\rangle=\lambda_{\mathrm{q}}^{-4} \lambda_{\mathrm{s}}^{-2} \lambda_{\mathrm{Q}}^{ \pm 2} .
\end{aligned}
$$

$\lambda_{\mathrm{s}}$ by itself is, by virtue of strangeness conservation, a function of $\lambda_{\mathrm{b}}$ and $\lambda_{\mathrm{Q}}$ (compare eq. (6.10)). The measured $\pi^{+} / \pi^{-}$ratio may be first used in order to fix $\lambda_{\mathrm{Q}}$ which describes the up-down quark asymmetry. We have:

$$
\pi^{+} / \pi^{-} \simeq \lambda_{\mathrm{Q}}^{2}
$$

Indeed, the experimental data for (pp reactions) can be satisfactorily represented by the functional form

$$
\lambda_{\mathrm{Q}}^{2}=\pi^{+} / \pi^{-}=\exp (2 x): \quad \text { pp reactions }
$$

in the region $0.2 \leq x \leq 0.7$.

The assumption (6.13) implies, in turn

$$
\begin{aligned}
& \lambda_{\mathrm{b}}^{2}=\exp \left(2 \mu_{\mathrm{b}} / T\right)=\exp \left(2 x m_{\mathrm{N}} / T\right) \\
& \left\{\begin{array}{lll}
=\exp (10 x) ; & T=175 \mathrm{MeV}: & \text { pp reactions } \\
=\exp (12.5 x) ; & T=150 \mathrm{MeV}: & \text { pN reactions }
\end{array}\right.
\end{aligned}
$$

where, as indicated, a range of the temperature parameter $T$ was taken above and below the value of $160 \mathrm{MeV}$. A similar change between $\mathrm{pp}$ and $\mathrm{pN}$ reactions in $\lambda_{\mathrm{Q}}$ is required and we will also use:

$$
\lambda_{\mathrm{Q}}^{2}=\exp (2.2 x): \quad \mathrm{pN} \text { reactions }
$$

In fig. 6.4 we compare the so calculated and the measured strange particle ratios $\left[(a) \mathrm{K}^{+} / \mathrm{K}^{-}\right.$in $\mathrm{p}-\mathrm{p}$ collisions; (b) $\mathrm{K}^{+} / \mathrm{K}^{-}$in $\mathrm{p}-\mathrm{N}$ collisions; (c) $\bar{\Lambda} / \Lambda$ in $\mathrm{p}-\mathrm{N}$ collisions and (d) $\overline{\mathrm{p}} \pi^{+} / \mathrm{p} \pi^{-}$in $\mathrm{p}-\mathrm{N}$ collisions]. 

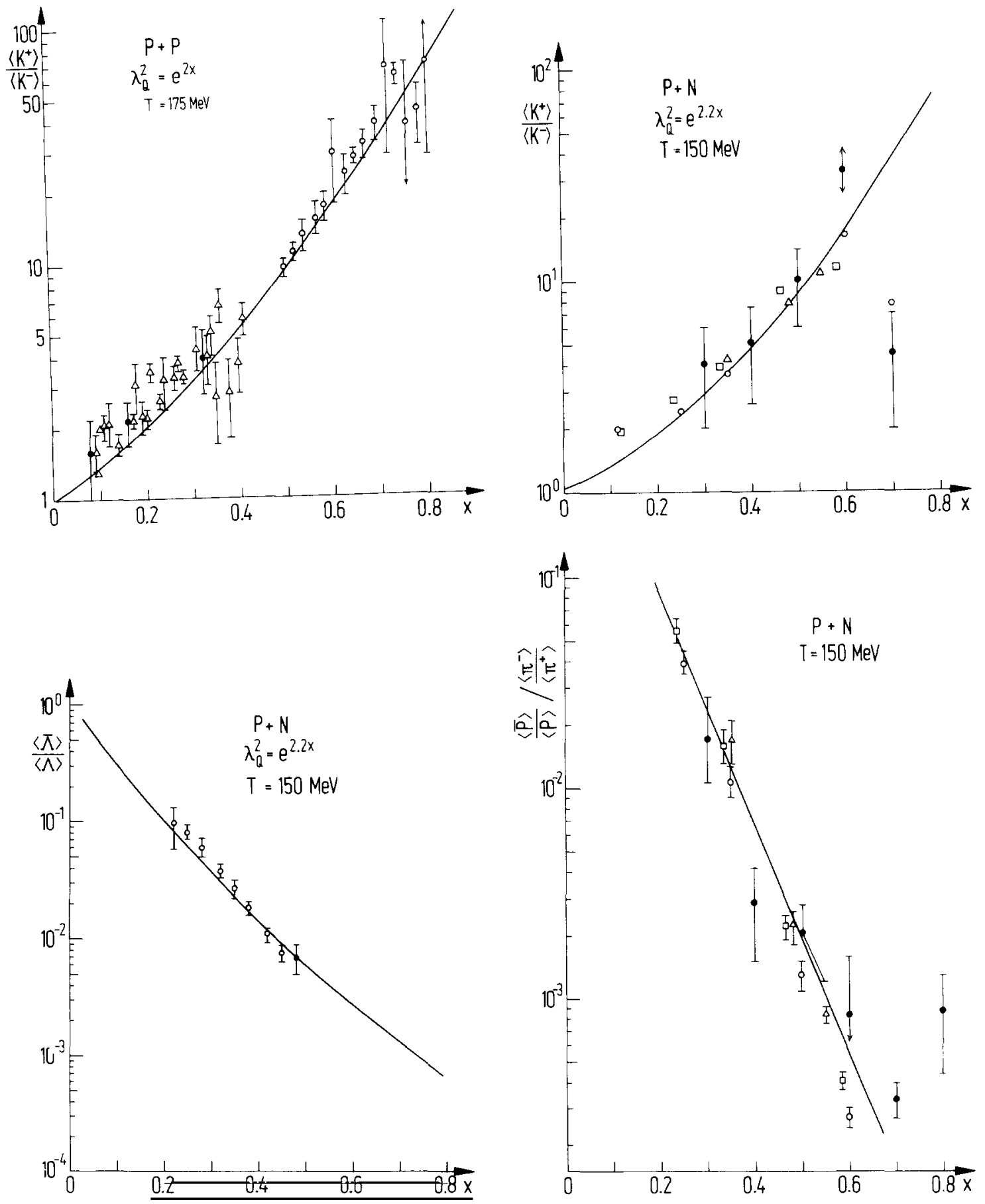

Fig. 6.4. Particle abundance ratios as function of $x$ in $\mathrm{pp}$ and $\mathrm{pN}$ collisions. Drawn lines are our calculated results with $T^{(\mathrm{pp})}=175 \mathrm{MeV}$ and $\lambda_{\mathrm{Q}}^{(\mathrm{pp})}=\exp (2 x)$ or $\lambda_{\mathrm{Q}}^{(\mathrm{pN})}=\exp (2.2 x)$ and $T^{(\mathrm{pN})}=150 \mathrm{MeV}$. (a) $\mathrm{K}^{+} / \mathrm{K}^{-}(\mathrm{pp})$ : ref. [Ca74] $(\triangle)$; ref. [Ro75] $(\cdot)$; ref. [Si78] (O). Data averaged over range of transverse momenta and $\sqrt{s}$. (b) $\mathrm{K}^{+} / \mathrm{K}^{-}(\mathrm{pBe})$; ref. [Ba74] $\left(\mathrm{O}\right.$, at $\left.p_{\text {proj }}=200 \mathrm{GeV} / c\right)$; ref. [Ba83] $\left(O\right.$ at $\left.p_{\text {proj }}=300 \mathrm{GeV} / c\right)$; refs. $\left[\right.$ Bo79, Bo80] $\left(\triangle, \mathrm{pBeO}\right.$ at $\left.p_{\text {proj }}=210 \mathrm{GeV} / c\right)$; refs. [Bo79, Bo80] $\left(\cdot\right.$, pA for various $\left.\mathrm{A}, p_{\text {proj }}=100 \mathrm{GeV} / c\right)$. (c) $\left(\bar{\Lambda}+\Sigma^{0}\right)(\mathrm{pBe})$, ref. [Sc78]. (d) double ratio $(\overrightarrow{\mathrm{p}} / \mathrm{p}):\left(\pi^{-} / \pi^{+}\right)$refs. $[\mathrm{Ba83}, \mathrm{Bo} 79, \mathrm{Bo80}]$. 
Note in (d) that the double ratio $(\overline{\mathrm{p}} / \mathrm{p}) /\left(\pi^{-} / \pi^{+}\right)$, which is equal to $\lambda_{\mathrm{b}}^{-2}$ and therefore provides an independent consistency check for the assumed important relation (6.13), gives a satisfactory agreement with data (fig. 6.4d). Taking any other power of $x$ in eq. (6.13) would destroy the visible exponential behaviour of this double ratio as a function of $x$.

Consider now the measured multistrange antiparticle to particle ratios [GKR84]. The comparison with data of Bourqin et al. [Bo79, Bo80] is shown in fig. 6.5 where the predictions are indicated by full circles. As a function of strangeness $(S=1,2,3)$, the data are relatively well described by relative abundances obtained in the framework of the hadronic gas model.

At first sight it is a surprising fact that the absolute experimental mean multiplicities of antistrange hyperons are compatible with the abundances we obtain from hadronic gas in chemical equilibrium at a temperature of $150 \mathrm{MeV}$ and a volume of about $1 \mathrm{fm}^{3}$, when further assuming that these particles are mainly produced in the central region of rapidity where $\mu_{\mathrm{b}}=0$. From our 'approach to equilibrium' analysis in the previous section, we know that often less than $10^{-3}$ of the strange antibaryonic phase space abundance is excited by reactions in the hadron gas phase. Further, we note that the $\bar{\Omega}$ abundance seen would require an active gas volume of $\sim 10^{4} \mathrm{fm}^{3}$ in order to be generated in the hadronic gas phase during the reaction time $10^{-23} \mathrm{~s}$. Thus, we must conclude that the relatively high abundance of antistrange hyperons observed has not been produced by hadronic gas reactions but that it must originate from reactions between quarks and gluons in the perturbative vacuum, with the structure of the intermediate state being similar to a quark-gluon plasma. This argument would not be consistent, though, if particle spectra did not just happen to have transverse momentum distributions corresponding to a high degree of thermalization, and in particular, a temperature of about $160 \mathrm{MeV}$ [Ha65, Ha68]. Further discussion of experimental facts suggesting this viewpoint is given by Koch and Rafelski [KRa85].

We now consider briefly the available sparse data on strange particle production in nucleus-nucleus collisions at several GeV/A lab kinetic energy [Ha81, Sch82, Sh82, An84]. These data cannot be described by hadronic gas phase space saturated particle abundances [ASS81, As81, Ko81, AS81, ABS84, RK80, Ra81, Zv84, ZS84, CL84, BI84, Bi82, MuK83, Ko83, Ko84]. This implies that at these

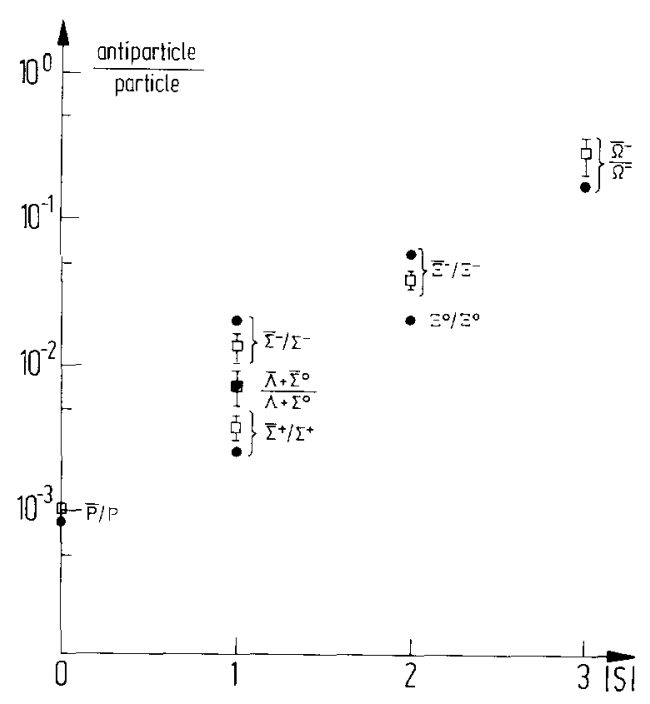

Fig. 6.5. Antibaryon to baryon ratio for p-Be collision as function of strangeness $S$ for $x=0.48$, ref. [Bo79]. Our calculated points are indicated by a full circle $\left[T=150 \mathrm{MeV}, \lambda_{\mathrm{Q}}=\exp (2.2 x)\right]$. 

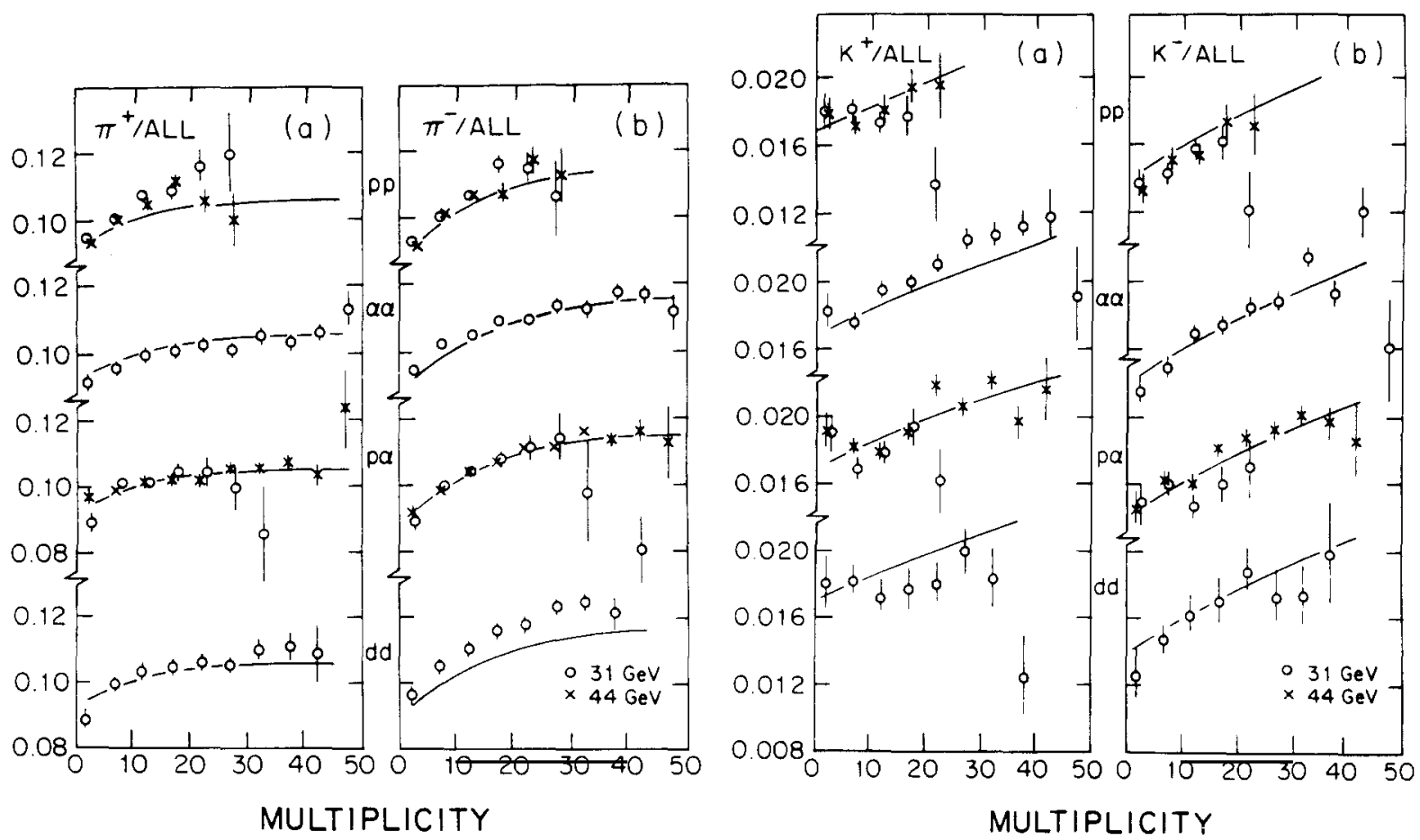

Fig. 6.6. Abundance of charged pions and kaons in $\mathrm{pp}, \alpha \alpha, \mathrm{p} \alpha$ and dd reactions at the CERN intersecting storage rings involving centre-of-mass energies $\sqrt{s}=31.5$ and $44 \mathrm{GeV}$ per nucleon. The $\pi / \mathrm{K}$ ratio of 11 percent is nearly constant. (From [Ak85].)

relatively low nuclear energies strange particle production is still dominated by reaction processes between individual hadrons, which therefore lead to particle abundances far below strange phase space saturation for hadronic gas models. Even at the much higher energies available at the ISR at CERN, light-ion reactions $(\mathrm{dd}, \alpha \alpha)$ have been shown to exhibit no spectacular increase in kaon versus pion production ([Ak85], see fig. 6.6). This indicates that quark matter has not been formed which would have enhanced the rate of strangeness abundance formation. Experiments with heavier projectiles have to show whether this is due to the smallness of the employed nuclei $(d, \alpha)$ or due to a still insufficiently high bombarding energy. However, even in the reactions mentioned above it is found that abundance ratios of strange particles obey the laws of the relative chemical equilibrium - that is strangeness generated is rapidly redistributed among the final state hadrons [KRG83]. A method for statistical description of relative chemical equilibrium is discussed in the following subsection.

In this subsection we have presented a number of arguments demonstrating the presence of the action of QCD colour degrees of freedom in various hadron-hadron as well as hadron-nucleus collisions [KRa85]. In particular, the question of why the reaction products display such a high degree of thermal and chemical equilibration could be qualitatively and plausibly explained by this hypothesis, founding a working base for statistical models of hadronic reactions.

\subsection{Relative chemical equilibrium}

It is possible to modify the grand canonical treatment of section 6.1 slightly to allow for relative equilibrium rather than for absolute chemical equilibrium only. As long as effects from weak 
interactions can be neglected, i.e. for time scales much shorter than $10^{-10} \mathrm{~s}$, the total strangeness must vanish. In the absence of absolute chemical equilibrium, the fugacities $\lambda_{\mathrm{s}}$ and $\lambda_{\overline{\mathrm{s}}}$ that control the hadronic content on strange quarks and antiquarks, respectively, are no longer related through the condition $\lambda_{\overline{\mathrm{s}}}=\lambda_{\mathrm{s}}^{-1}$. Rather, one has to assume

$$
\lambda_{\overline{\mathrm{s}}}=f \lambda_{\mathrm{s}}^{-1}
$$

with the new parameter $f$ controlling the deviation from absolute chemical equilibrium, which corresponds to the value $f=1$.

The partition function in the Boltzmann approximation is:

$$
\ln Z^{\mathrm{s}}=Z_{\mathrm{K}}\left(\lambda_{\mathrm{s}} \lambda_{\mathrm{q}}^{-1}+\lambda_{\mathrm{q}} \lambda_{\overline{\mathrm{s}}}\right)+Z Z_{\mathrm{Y}}\left(\lambda_{\mathrm{s}} \lambda_{\mathrm{q}}^{2}+\lambda_{\overline{\mathrm{s}}} \lambda_{\mathrm{q}}^{-2}\right)+Z_{\Xi}\left(\lambda_{\mathrm{s}}^{2} \lambda_{\mathrm{q}}+\lambda_{\overline{\mathrm{s}}}^{2} \lambda_{\mathrm{q}}^{-1}\right)+Z_{\Omega}\left(\lambda_{\overline{\mathrm{s}}}^{3}\right)+Z_{\phi} \lambda_{\mathrm{s}} \lambda_{\overline{\mathrm{s}}},
$$

where $Z_{j}$ are the one-particle functions, eq. (6.3). The average total number of strange quarks contained in the hadrons is

$$
\left\langle n_{\mathrm{s}}\right\rangle=\lambda_{\mathrm{s}} \frac{\partial}{\partial \lambda_{\mathrm{s}}} \ln Z^{\mathrm{s}}=Z_{\mathrm{K}} \lambda_{\mathrm{s}} \lambda_{\mathrm{q}}^{-1}+Z_{\mathrm{Y}} \lambda_{\mathrm{s}} \lambda_{\mathrm{q}}^{2}+2 Z_{\Xi} \lambda_{\mathrm{s}}^{2} \lambda_{\mathrm{q}}+3 Z_{\Omega} \lambda_{\mathrm{s}}^{3}+Z_{\phi} \lambda_{\mathrm{s}} \lambda_{\overline{\mathrm{s}}},
$$

whereas the total number of strange antiquarks is given by

$$
\left\langle n_{\overline{\mathrm{s}}}\right\rangle=\lambda_{\overline{\mathrm{s}}} \frac{\partial}{\partial \lambda_{\overline{\mathrm{s}}}} \ln Z^{\mathrm{s}}=Z_{\mathrm{K}} \lambda_{\overline{\mathrm{s}}} \lambda_{\mathrm{q}}+Z_{\mathrm{Y}} \lambda_{\overline{\mathrm{s}}} \lambda_{\mathrm{q}}^{-2}+2 Z_{\Xi} \lambda_{\overline{\mathrm{s}}}^{2} \lambda_{\mathrm{q}}^{-1}+3 Z_{\Omega} \lambda_{\overline{\mathrm{s}}}^{3}+Z_{\phi} \lambda_{\mathrm{s}} \lambda_{\overline{\mathrm{s}}} .
$$

Since we now have two independent parameters, $\lambda_{\mathrm{s}}$ and $\lambda_{\overline{\mathrm{s}}}$, we can fix $\left\langle n_{\mathrm{s}}\right\rangle$ and $\left\langle n_{\overline{\mathrm{s}}}\right\rangle$ independently. As discussed above, the condition of vanishing net strangeness has to be imposed

$$
0=\left\langle n_{\mathrm{s}}\right\rangle-\left\langle n_{\overline{\mathrm{s}}}\right\rangle=Z_{\mathrm{K}}\left(\lambda_{\mathrm{s}} \lambda_{\mathrm{q}}^{-1}-\lambda_{\overline{\mathrm{s}}} \lambda_{\mathrm{q}}\right)+Z_{\mathrm{Y}}\left(\lambda_{\mathrm{s}} \lambda_{\mathrm{q}}^{2}-\lambda_{\overline{\mathrm{s}}} \lambda_{\mathrm{q}}^{-2}\right)+2 Z_{\Xi}\left(\lambda_{\mathrm{s}}^{2} \lambda_{\mathrm{q}}-\lambda_{\overline{\mathrm{s}}}^{2} \lambda_{\mathrm{q}}^{-1}\right)+3 Z_{\Omega}\left(\lambda_{\mathrm{s}}^{3}-\lambda_{\overline{\mathrm{s}}}^{3}\right) .
$$

Considering the singly strange hadrons which in most instances dominate the strangeness abundance, we find the condition

$$
f=\lambda_{\mathrm{s}} \lambda_{\tilde{\mathrm{s}}} \sim \frac{\lambda_{\mathrm{s}}^{2}\left(Z_{\mathrm{K}}+\lambda_{\mathrm{q}}^{3} Z_{\mathrm{Y}}\right)}{\lambda_{\mathrm{q}}^{2}\left(Z_{\mathrm{K}}+\lambda_{\mathrm{q}}^{-3} Z_{\mathrm{Y}}\right)}=\gamma^{2} \frac{Z_{\mathrm{K}}+\lambda_{\mathrm{v}} Z_{\mathrm{Y}}}{Z_{\mathrm{K}}+Z_{\mathrm{Y}} / \lambda_{\mathrm{b}}},
$$

where $\gamma=\lambda_{\mathrm{s}} / \lambda_{\mathrm{q}}$ and $\lambda_{\mathrm{b}}=\lambda_{\mathrm{q}}^{3}$ as before, cf. eqs. (6.5) and (6.10).

Ratios of particle numbers are now easily deduced from eqs. (6.21), e.g.

$$
\begin{aligned}
& \left\langle n_{\bar{\Lambda}}\right\rangle /\left\langle n_{\Lambda}\right\rangle=\lambda_{\overline{\mathrm{s}}} \lambda_{\mathrm{q}}^{-2} / \lambda_{\mathrm{s}} \lambda_{\mathrm{q}}^{2}=f \gamma^{-2} \lambda_{\mathrm{b}}^{-2} \\
& \left\langle n_{\Xi}\right\rangle /\left\langle n_{\Xi}\right\rangle=\lambda_{\overline{\mathrm{s}}}^{2} \lambda_{\mathrm{q}}^{-1} / \lambda_{\mathrm{s}}^{2} \lambda_{\mathrm{q}}=f^{2} \gamma^{-4} \lambda_{\mathrm{b}}^{-2}, \quad \text { etc. }
\end{aligned}
$$


The relations are obtained from those valid at absolute chemical equilibrium, eqs. (6.12), by the simple substitution $\gamma^{-2} \rightarrow f \gamma^{-2}$. From eq. (6.23), however, it is obvious that $f \gamma^{-2}$ numerically has the same value as previously $\gamma^{-2}$, eq. (6.10), which should now be denoted $\gamma_{\mathrm{eq}}$. Hence the ratios of particles are the same as found in absolute equilibrium even though this state has not been reached. Thus, the same 'relative' chemical equilibrium is fully justified.

We now proceed to fix the value of $\lambda_{\mathrm{s}}$, i.e. $\gamma$. Ignoring again the influence of multistrange hadrons, we find from eq. (6.21a)

$$
\left\langle n_{\mathrm{s}}\right\rangle \sim \gamma\left(Z_{\mathrm{K}}+\lambda_{\mathrm{b}} Z_{\mathrm{Y}}\right)
$$

Given some arbitrary value of $\left\langle n_{\mathrm{s}}\right\rangle$, we can use eq. (6.25) to fix $\gamma$.

This then allows us to compute the value of $f$. Inserting eq. (6.25) into (6.23) we find

$$
f=\frac{\left\langle n_{\mathrm{s}}\right\rangle^{2}}{\left(Z_{\mathrm{K}}+Z_{\mathrm{Y}} / \lambda_{\mathrm{b}}\right)\left(Z_{\mathrm{K}}+\lambda_{\mathrm{b}} Z_{\mathrm{Y}}\right)}=\left[\frac{\left\langle n_{\mathrm{s}}\right\rangle}{\left\langle n_{\mathrm{s}}^{\mathrm{eq}}\right\rangle}\right]^{2}
$$

where the last equality follows from the consideration of the equilibrium limit $f \rightarrow 1$. We further note that according to eq. $(6.23)$

$$
\gamma=f^{1 / 2} \gamma_{\mathrm{eq}}
$$

where, as mentioned, $\gamma_{\text {eq }}$ is as given by eq. (6.10). As we have already shown, the ratios of strange particle abundances do not change in relative chemical equilibrium but are the same as in absolute chemical equilibrium. Equation (6.26) further shows that the absolute abundances of strange hadrons are proportional to the actual total abundance $\left\langle n_{\mathrm{s}}\right\rangle$. In detail consider for example the hyperon abundance:

$$
\begin{aligned}
\left\langle n_{\mathrm{Y}}\right\rangle & =Z_{\mathrm{Y}} \lambda_{\mathrm{s}} \lambda_{\mathrm{q}}^{2}=Z_{\mathrm{Y}} \gamma \lambda_{\mathrm{b}} \\
& =\frac{\left\langle n_{\mathrm{s}}\right\rangle}{\left\langle n_{\mathrm{s}}^{\mathrm{eq}}\right\rangle} Z_{\mathrm{Y}} \lambda_{\mathrm{b}} \gamma_{\mathrm{eq}}=\frac{\left\langle n_{\mathrm{s}}\right\rangle}{\left\langle n_{\mathrm{s}}^{\mathrm{eq}}\right\rangle}\left\langle n_{\mathrm{Y}}^{\mathrm{eq}}\right\rangle .
\end{aligned}
$$

At this point it is worthwhile to appreciate that this behaviour is borne out by results shown in fig. 4.5 . There is clearly a visible difference by a factor of two between strange baryon abundances in (equilibrated) hadronic gas (full lines) and the recombination abundances from plasma (dashed lines). However, our simple approach does not account for the more complex behaviour of strange antibaryons, where at high baryon density orders of magnitude differences arise, cf. figs. 4.4 and 4.6. Recall again that equilibrated hadronic gas is shown for comparison - its effective appearance is a signal for quark-gluon plasma or precritical phenomena [KRa85].

\subsection{Measurement of the baryochemical potential}

As we have seen in the previous two subsections, the baryochemical potential is of similar physical importance as the temperature of the hot hadronic matter - it is hence of great interest to explore all the possible avenues which would permit its measurement. Perhaps the simplest and most sensitive 
approach proposed by Koch et al. [KRG83] is to study the distribution of s-quarks among mesons and baryons. The existence of $\bar{K}-\mathrm{N}$ resonances assures that this process is a rapid one and that whatever s-quark abundance is present will be shared according to the laws of relative chemical equilibrium. Naturally, as is the case with the measurement of temperature by observation of shapes of particle spectra, we will nearly always measure a 'freeze out' chemical potential, that is, some effective average over the last stages of the evolution of the hadronic fireball.

A first promising case is the ratio of $\mathrm{K}^{+}$to $\mathrm{K}^{-}$abundances: both can be observed in the same experimental set up. $\mathrm{K}^{+}$(together with $\mathrm{K}^{0}$ ) is the major carrier of $\bar{s}$-quarks, while $\mathrm{K}^{-}$(together with $\mathrm{K}^{0}$ ) contains only a fraction of the s-abundance, the balance being with the hyperons. Thus the $\mathrm{K}^{+}$ provides the normalisation for the $\mathrm{K}^{-}$abundance which depends sensitively on the baryon chemical balance. In fig. 6.7 this ratio is shown as follows from the simple relationship

$$
n_{\mathrm{K}^{+}} / n_{\mathrm{K}^{-}}=\lambda_{\mathrm{s}}^{-2} \lambda_{\mathrm{q}}^{2}
$$

using $\lambda_{\mathrm{s}}$ given by eq. (6.10) and neglecting the effect of electric charge discussed in eq. (6.14). Since $\mathrm{K}^{+}$ will have, in general, fewer interactions than the $\mathrm{K}^{-}$, we expect that an important correction must be applied in actual circumstances in order to account for effective cooling of $\mathrm{K}^{-}$due to expansion of the hadronic gas.

The ratio of abundance of $\mathrm{K}^{-}$to $\Lambda$ seems to be the next logical case to study as both $\mathrm{K}^{-}$and $\Lambda$ will suffer the expansion cooling.

One should notice, however, that $\Sigma^{0}$ will decay quite rapidly into $\Lambda$ and will consequently contaminate the measurements - it will be difficult to judge how many $\Lambda$ 's really are $\Sigma^{0}$, the latter one originating in part also from charge exchange reactions of $\Sigma^{ \pm}$. Further $\Lambda$ 's arise from decay of multiply
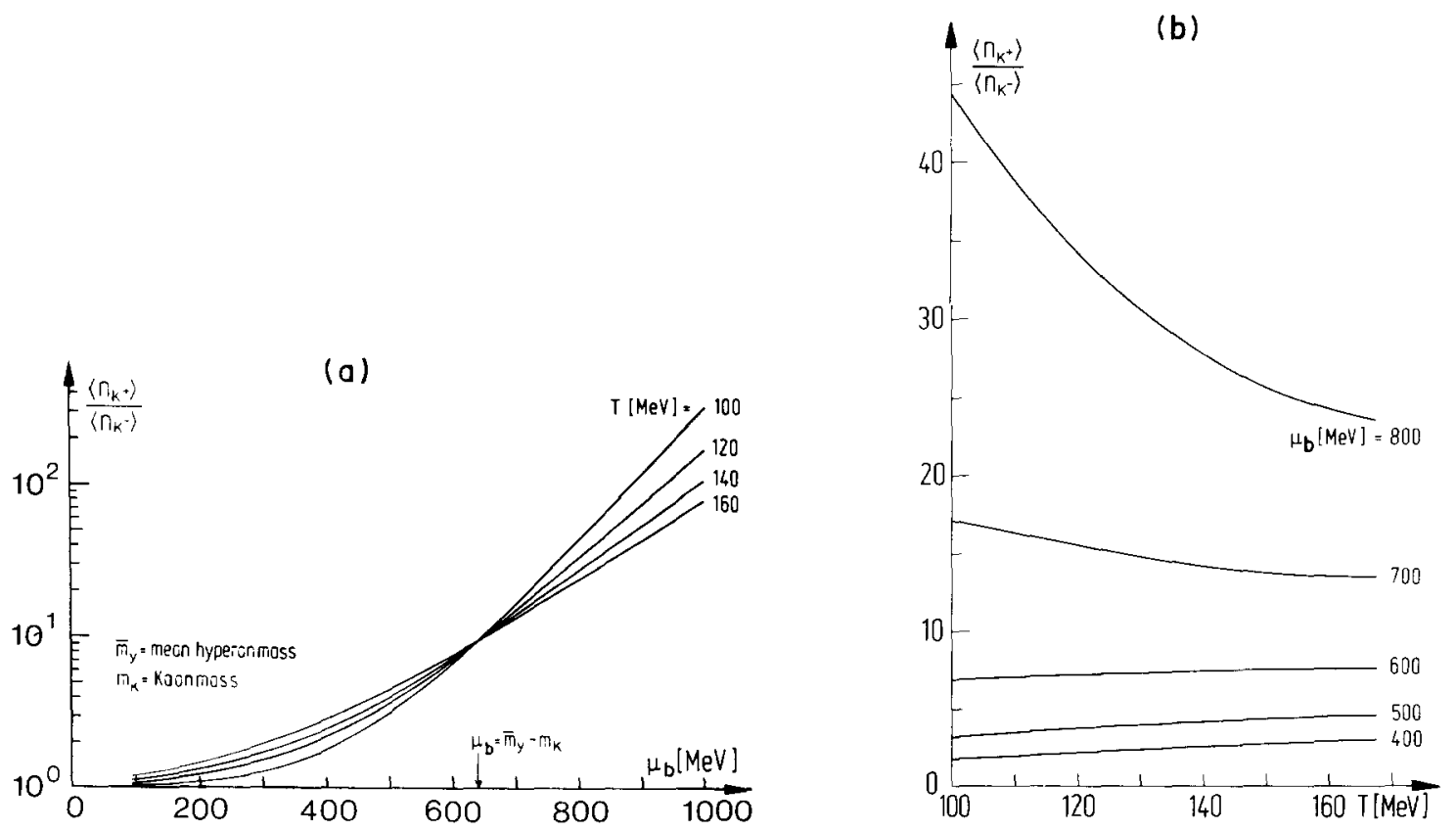

Fig. 6.7. The ratio $\left\langle n_{\mathrm{K}^{+}} / n_{\mathrm{K}^{-}}\right\rangle$(a) as a function of the baryochemical potential for several temperatures $T$ and (b) as a function of $T$ for several values of $\mu_{\mathrm{b}}$. 


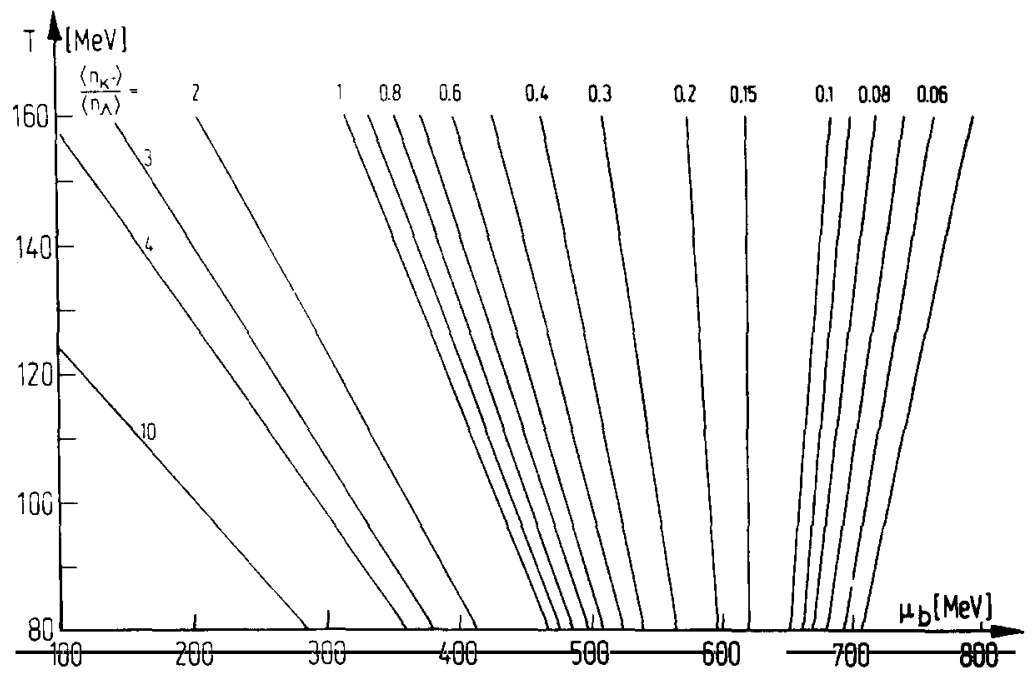

Fig. 6.8. Contours of abundance ratio $\left\langle n_{\mathrm{K}^{-}}\right\rangle /\left\langle n_{\mathrm{A}}\right\rangle$ in the $\mu_{\mathrm{b}}-T$ plane.

strange baryons when such decay occur sufficiently rapidly in order for the decay cascade to be hidden in the reaction vertex. However, we can presume that the $\Lambda$-abundance will dominate these effects which can be corrected for in an experimental situation. Hence, we show in fig. 6.8 the contours for the $\mathrm{K}^{-} / \Lambda$ ratio expected according to the simple phase space measure (see subsection 6.1 ),

$$
n_{\mathrm{K}} / n_{\Lambda}=\lambda_{\mathrm{s}}^{-2} \lambda^{-1} W\left(x_{\mathrm{K}}\right) / W\left(x_{\Lambda}\right)
$$

and $\lambda_{\mathrm{s}}$ as given by eq. (6.10) may be used again here.

The temperature range considered is below $160 \mathrm{MeV}$, since both $\mathrm{K}^{-}$and $\Lambda$ will strongly interact in the expansion phase of the hadronic gas and be relatively 'cold' at the freeze-out point.

\section{Strangeness evolution in an expanding fireball}

\subsection{Fireball scenario}

The hadronic fireball formed in a nucleus-nucleus collision at high energy is not a static object, but expands under the influence of its internal pressure subject to its inertia. We now consider the equation of motion for strangeness carrying particles in such an environment. There are three possible phases that must be accounted for: (i) a pure quark-gluon plasma phase, (ii) a pure hadronic phase, and (iii) a mixed phase containing quark-gluon matter and hadronic matter side by side. We shall commence with the discussion of the strangeness evolution in the mixed (coexistence) phase, since the other two cases follow immediately in the appropriate limit.

Following Kapusta and Mekjian [KM85] we consider a volume element $V$, of which a fraction $f_{\mathrm{Q}}=V_{\mathrm{Q}} / V$ is made up of quark matter, while the remaining fraction $f_{\mathrm{H}}=V_{\mathrm{H}} / V$ consists of hadronic matter, i.e.:

$$
V=V_{\mathrm{Q}}+V_{\mathrm{H}}, \quad f_{\mathrm{Q}}+f_{\mathrm{H}}=1
$$


The volume $V$ expands in time

$$
\mathrm{d} V=\frac{\mathrm{d} V}{\mathrm{~d} t} \mathrm{~d} t=\mathrm{d} V_{\mathrm{Q}}+\mathrm{d} V_{\mathrm{H}}
$$

There are two mechanisms that lead to a change in volume occupied by the two phases. The volume may change due to a hydrodynamical expansion at nearly constant entropy. Alternatively the volumes can change due to mutual exchange of particles, i.e. entropy, between the two phases. This latter process is associated with the quark-gluon fragmentation process we have discussed in section 4 .

We shall assume that in the expansion stage phase conversion proceeds only from the plasma phase into the hadronic phase but not vice versa, so that the plasma volume monotonically decreases during the existence of a mixed phase. Simultaneously, the volume of the hadronic phase continuously increases, due to the accumulation of hadronic particles as well as due to (hydrodynamical) volume expansion.

Thus the change in volume per time interval $\mathrm{d} t$ reads

$$
\mathrm{d} V_{\mathrm{Q}}=\mathrm{d} V_{\mathrm{Q}}^{\mathrm{e}}-\mathrm{d} V^{\mathrm{p}}, \quad \mathrm{d} V_{\mathrm{H}}=\mathrm{d} V_{\mathrm{H}}^{\mathrm{e}}+\mathrm{d} V^{\mathrm{p}},
$$

where the $\mathrm{d} V_{\mathrm{H}, \mathrm{Q}}^{\mathrm{e}}$ denote volume expansion (at fixed entropy) while $\mathrm{d} V^{\mathrm{p}}$ stands for the volume change due to particle exchange (indices ' $e$ ' stand for expansion and 'p' for particle exchange). Before and after the hadronization process we have $\mathrm{d} V_{\mathrm{p}}=0$; during the existence of the mixed phase, we put $\mathrm{d} V_{\mathrm{Q}}^{\mathrm{e}}=0$, as stated above. We focus our attention now on the time evolution of the mixed phase. We must first establish a relation between $\mathrm{d} V_{\mathrm{Q}}$ and $\mathrm{d} V_{\mathrm{H}}$. This is provided by the assumption that there is no significant production of entropy, i.e. that the increase in entropy in the hadronic phase $\mathrm{d} S_{\mathrm{H}}$ is balanced by the loss of entropy from the quark-gluon plasma $\mathrm{d} S_{\mathrm{Q}}$ due to particle loss:

$$
\mathrm{d} S_{\mathrm{Q}}=s_{\mathrm{Q}} \mathrm{d} V_{\mathrm{Q}}=-s_{\mathrm{H}} \mathrm{d} V_{\mathrm{H}}=-\mathrm{d} S_{\mathrm{H}},
$$

where $s_{\mathrm{Q}}$ and $s_{\mathrm{H}}$ are the associated entropy densities. In the non-interacting quark-gluon plasma we have

$$
s_{\mathrm{Q}}=\frac{64}{45} \pi^{2} T^{3}+2\left(\mu_{\mathrm{b}} / 3\right)^{2} T
$$

where $\mu_{\mathrm{b}}$ is as usual the baryon chemical potential. The entropy density in the hadronic phase is approximately

$$
s_{\mathrm{H}}=\frac{2}{15} \pi^{2} T^{3}+4\left[\left(5+\frac{2 m_{\mathrm{N}}}{T}\right) \operatorname{ch}\left(\frac{\mu_{\mathrm{b}}}{T}\right)-\frac{2 \mu_{\mathrm{b}}}{T} \operatorname{sh}\left(\frac{\mu_{\mathrm{b}}}{T}\right)\right]\left(\frac{m_{\mathrm{N}} T}{2 \pi}\right)^{3 / 2} \exp \left(-\beta m_{\mathrm{N}}\right)
$$

where $m_{\mathrm{N}}$ is the nucleon mass. As the Boltzmann approximation has been used to derive eq. (7.6), we must require that $\left(\mu_{\mathrm{b}}-m_{\mathrm{N}}\right)<T$ for its validity.

Let us here, in the first consideration, neglect the influence of a nonvanishing baryon chemical potential $\mu_{\mathrm{b}}$ and of the baryonic contribution to $s_{\mathrm{H}}$. Equation (7.4) then describes the increase in hadronic volume due to gradual conversion of quark-gluon plasma into a pion gas as according to the simple relation:

$$
\mathrm{d} V_{\mathrm{H}}=-\left(s_{\mathrm{Q}} / s_{\mathrm{H}}\right) \mathrm{d} V_{\mathrm{Q}}=-\frac{32}{3} \mathrm{~d} V_{\mathrm{Q}} .
$$


This means that in the end after the conversion of the quark-gluon plasma to pionic gas, the latter must occupy a volume that is at about ten times larger in order to conserve entropy! This increase in volume results in a dilution of strange quarks among light quarks, which is somewhat mitigated by the creation of additional strange quark pairs in the fragmentation process discussed in section 4.2 . The dilution effect was observed by Redlich [Re85] in comparing equilibrium strangeness abundancies in the quark-gluon and hadronic gas phases. Equating the total baryon number in the two phases (at equal baryochemical potential $\mu_{\mathrm{b}}$ ) he calculated the ratio of the number of strange particles, which is shown in fig. 7.1 as a function of $\mu_{\mathrm{b}}$ at $T=160 \mathrm{MeV}$. At $\mu_{\mathrm{b}}=0$ the ratio of volumes is $V_{\mathrm{H}}: V_{\mathrm{Q}}=6.5$.

As is evident from fig. 7.1, the volume dilution leads to a rather small value of the enhancement of strangeness in the quark-gluon plasma. The factor $1.5 \pm 0.2$ would render experimental use of strangeness as signal for formation of a deconfined state useless, if chemical equilibrium concentration of strange particles were really reached in nuclear reactions proceeding solely through the hadronic gas phase. However, as shown in section 5, in the kinetic treatment of the evolution of a hot hadronic gas, strangeness equilibrium densities are missed by a large factor. As a consequence, the dynamical enhancement factor for strangeness production in the quark-gluon plasma phase is much larger than that shown in fig. 7.1. According to the results depicted in fig. 5.4, the additional dynamical enhancement amounts to a factor 5 or more for a lifetime of the hadronic fireball of $10 \mathrm{fm} / c$.

We now proceed to explore the consequences of a hadronization scenario in which a large volume jump occurs. Since the phase transition and volume growth is, in reality, accomplished gradually through participation of numerous intermediate hadronic states, we will turn further below to consider, in addition to quark recombination, the quark and gluon fragmentation mechanism introduced in section 4 . The latter effect is required in order to generate the additional hadronic particles that take up the entropy content of the quark-gluon plasma and fill the larger hadronic volume.

We are mainly interested in the fate of strange quarks. The number of strange quarks in the plasma phase changes because of two reasons: on the one hand, strangeness producing reactions make it approach the equilibrium abundance according to the law:

$$
\mathrm{d} N_{\mathrm{s}}^{\mathrm{Q}}=A_{\mathrm{g}}\left[1-\left(N_{\mathrm{s}} / N_{\mathrm{s}}^{\mathrm{eq}}\right)^{2}\right] V_{\mathrm{Q}} \mathrm{d} t \equiv R_{\mathrm{s}} V_{\mathrm{Q}} \mathrm{d} t
$$

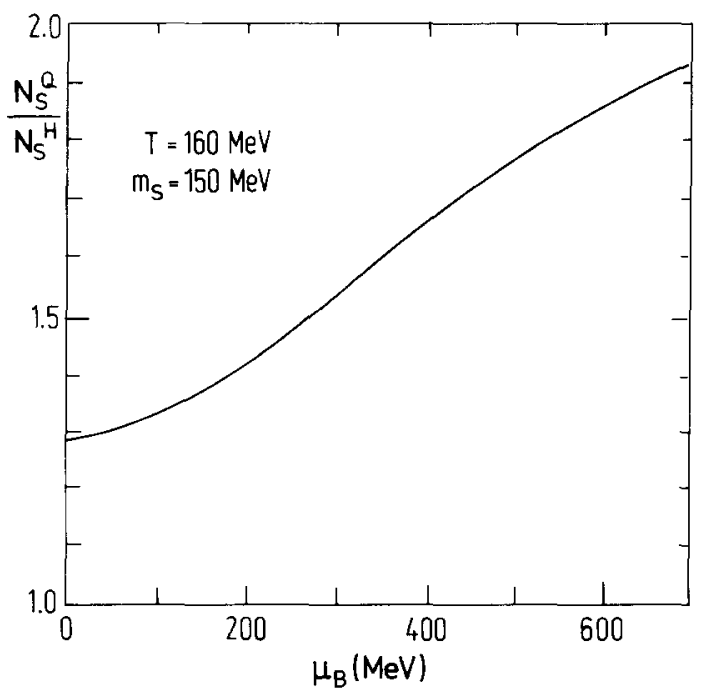

Fig. 7.1. Ratio of the number of strange particles in quark-gluon plasma and hadronic gas containing the same net baryon number. The ratio is shown as a function of $\mu_{\mathrm{b}}$ (after [Re85]). 
where $A_{\mathrm{g}}$ is the rate constant (3.10) and $R_{\mathrm{s}}$ includes the effective reduction factor $\left[1-\left(N_{\mathrm{s}} / N_{\mathrm{s}}^{\mathrm{eq}}\right)^{2}\right]$. Furthermore, the number of strange quarks in the plasma phase changes due to their conversion into hadrons. We shall assume the loss of strangeness, $\mathrm{d} N_{\mathrm{s}}^{\mathrm{p}}$, is proportional to the loss of volume $\mathrm{d} V^{\mathrm{p}}$, i.e.

$$
\mathrm{d} N_{\mathrm{s}}^{\mathrm{p}}=\left(N_{\mathrm{s}} / V_{\mathrm{Q}}\right) \mathrm{d} V^{\mathrm{p}}=N_{\mathrm{s}} \mathrm{d}\left(\ln V_{\mathrm{Q}}\right)
$$

where we have assumed that $\mathrm{d} V^{\mathrm{p}}=\mathrm{d} V_{\mathrm{Q}}$ in the mixed plasma-hadron phase. The total change in strangeness content in a time interval $\mathrm{d} t$ is therefore

$$
\mathrm{d} N_{\mathrm{s}}=\mathrm{d} N_{\mathrm{s}}^{\mathrm{Q}}+\mathrm{d} N_{\mathrm{s}}^{\mathrm{p}}=R_{\mathrm{s}} V_{\mathrm{Q}} \mathrm{d} t+N_{\mathrm{s}} \mathrm{d}\left(\ln V_{\mathrm{Q}}\right) .
$$

Dividing by $V_{\mathrm{Q}} \mathrm{d} t$, and with

$$
N_{\mathrm{s}}=\rho_{\mathrm{s}} V_{\mathrm{Q}}
$$

where $\rho_{\mathrm{s}}$ is the density of strange quarks in the plasma phase, we find:

$$
\frac{\mathrm{d}}{\mathrm{d} t} \rho_{\mathrm{s}}=\frac{1}{V_{\mathrm{Q}}} \frac{\mathrm{d}}{\mathrm{d} t} N_{\mathrm{s}}-\rho_{\mathrm{s}} \frac{\mathrm{d}}{\mathrm{d} t}\left(\ln V_{\mathrm{Q}}\right)=R_{\mathrm{s}} .
$$

Equation (7.12) emphasises that there is no dilution term in the QGP strangeness density evolution equation because the density of strange quarks is assumed to change only due to particle reactions, but not due to hydrodynamical expansion of the volume occupied by the plasma phase. This fact is contained implicitly in our assumption $\mathrm{d} V_{\mathrm{Q}}^{\mathrm{e}}=0$ for the mixed phase.

Next, we consider the change in the number of strange particles, e.g. of antikaons $\mathrm{K}^{-}$or $\overline{\mathrm{K}}^{0}$, in the hadronic phase. It can change either through hadronic reactions

$$
\mathrm{d} N_{\overline{\mathrm{K}}}^{\mathrm{R}}=A_{\overline{\mathrm{K}}}\left[1-\left(N_{\overline{\mathrm{K}}} / N_{\overline{\mathrm{K}}}^{\mathrm{eq}}\right)^{2}\right] V_{\mathrm{H}} \mathrm{d} t \equiv R_{\overline{\mathrm{K}}} V_{\mathrm{H}} \mathrm{d} t
$$

or due to conversion of strange quarks from plasma into antikaons:

$$
\mathrm{d} N_{\overline{\mathrm{K}}}^{\mathrm{p}}=-p_{\overline{\mathrm{K}}} \mathrm{d} N_{\mathrm{s}}^{\mathrm{p}}=-p_{\overline{\mathrm{K}}} N_{\mathrm{s}} \mathrm{d}\left(\ln V_{\mathrm{Q}}\right)
$$

Here $p_{\overline{\mathrm{K}}}$ describes the probability for a hadronizing strange quark to end up in an antikaon rather than other hadron.

In the spirit of eq. (4.17b) we have

$$
-\mathrm{d} N_{\overline{\mathrm{K}}}^{\mathrm{p}} / \mathrm{d} N_{\mathrm{s}}^{\mathrm{p}}=p_{\overline{\mathrm{K}}}=\alpha \tilde{N}_{\overline{\mathrm{q}}} \tilde{N}_{\mathrm{s}} / N_{\mathrm{s}}
$$

understanding implicitly that the effective number of quarks, as determined by eqs. (4.16), should be used on the right hand side. We thus obtain

$$
\mathrm{d} N_{\overline{\mathrm{K}}}^{\mathrm{p}}=-\alpha \bar{N}_{\overline{\mathrm{q}}} \tilde{N}_{\mathrm{s}} \mathrm{d}\left(\ln V_{\mathrm{Q}}\right)
$$

where the constant $\alpha$ must be taken from eq. (4.22a). 
We can, finally, again replace particle numbers by particle densities,

$$
N_{\overline{\mathrm{K}}}=\rho_{\overline{\mathrm{K}}} V_{\mathrm{H}}
$$

and we find, combining eqs. (7.13) and (7.14):

$$
\frac{\mathrm{d} \rho_{\overline{\mathrm{K}}}}{\mathrm{d} t}=\frac{1}{V_{\mathrm{H}}}\left(\frac{\mathrm{d} N_{\overline{\mathrm{K}}}^{\mathrm{R}}}{\mathrm{d} t}+\frac{\mathrm{d} N_{\overline{\mathrm{K}}}^{\mathrm{p}}}{\mathrm{d} t}\right)-\rho_{\overline{\mathrm{K}}} \frac{\mathrm{d}}{\mathrm{d} t}\left(\ln V_{\mathrm{H}}\right)=R_{\overline{\mathrm{K}}}-\alpha \tilde{N}_{\overline{\mathrm{q}}} \tilde{\rho}_{\mathrm{s}} \frac{1}{V_{\mathrm{H}}} \frac{\mathrm{d} V_{\mathrm{Q}}}{\mathrm{d} t}-\rho_{\overline{\mathrm{K}}} \frac{\mathrm{d}}{\mathrm{d} t}\left(\ln V_{\mathrm{H}}\right) .
$$

It is now convenient to express $\mathrm{d} V_{\mathrm{Q}}$ by $\mathrm{d} V_{\mathrm{H}}$ with the help of eq. (7.7)

$$
\frac{\mathrm{d}}{\mathrm{d} t} \rho_{\overline{\mathrm{K}}}=R_{\overline{\mathrm{K}}}+\alpha \tilde{N}_{\overline{\mathrm{q}}} \tilde{\rho}_{\mathrm{s}} \frac{s_{\mathrm{H}}}{s_{\mathrm{Q}}} \frac{\mathrm{d}}{\mathrm{d} t}\left(\ln V_{\mathrm{H}}\right)-\rho_{\overline{\mathrm{K}}} \frac{\mathrm{d}}{\mathrm{d} t}\left(\ln V_{\mathrm{H}}\right)=R_{\overline{\mathrm{K}}}+\left(\alpha \bar{N}_{\overline{\mathrm{q}}} \tilde{\rho}_{\mathrm{s}} \frac{s_{\mathrm{H}}}{s_{\mathrm{Q}}}-\rho_{\overline{\mathrm{K}}}\right) \frac{\mathrm{d}}{\mathrm{d} t}\left(\ln V_{\mathrm{H}}\right) .
$$

The right hand side contains the reaction term, a feeding term reflecting on expressing creation of new kaons from the quark phase, and the dilution term due to expansion of the hadronic phase. Whereas loss term and dilution term exactly cancelled in eq. (7.12), this is not so here for the feeding and dilution terms.

For the sake of completeness, we record the evolution equation for the pure phases, which do not contain exchange terms. Then one has:

$$
\frac{\mathrm{d}}{\mathrm{d} t} \rho_{\mathrm{s}}=R_{\mathrm{s}}-\rho_{\mathrm{s}} \frac{\mathrm{d}}{\mathrm{d} t}(\ln V)
$$

for the pure quark-gluon phase, and

$$
\frac{\mathrm{d}}{\mathrm{d} t} \rho_{\overline{\mathrm{K}}}=R_{\overline{\mathrm{K}}}-\rho_{\overline{\mathrm{K}}} \frac{\mathrm{d}}{\mathrm{d} t}(\ln V)
$$

for the pure hadronic phase. The index ' $Q$ ' or ' $H$ ' is not needed for the volume then, since either $V=V_{\mathrm{Q}}$ or $V=V_{\mathrm{H}}$, depending on the phase.

We must now specify the initial conditions for the hadron densities in the mixed phase. Concentrating on antikaons, in the first moment of the mixed phase $N_{\overline{\mathrm{K}}}=V_{\mathrm{H}}=0$, so that the initial value for $\rho_{\overline{\mathrm{K}}}$ is not obvious. However, if we go back to eqs. (7.13) and (7.16), we find in the limit $V_{\mathrm{H}} \rightarrow 0$ : $\mathrm{d} N_{\overline{\mathrm{K}}}^{\mathrm{R}} / \mathrm{d} t=0$,

$$
\mathrm{d} N_{\overline{\mathrm{K}}}^{\mathrm{p}}=-\alpha \tilde{N}_{\overline{\mathrm{q}}} \tilde{N}_{\mathrm{s}} \mathrm{d}\left(\ln V_{\mathrm{Q}}\right)=\alpha \tilde{N}_{\overline{\mathrm{q}}} \tilde{\rho}_{\mathrm{s}} \frac{s_{\mathrm{H}}}{s_{\mathrm{Q}}} \mathrm{d} V_{\mathrm{H}}
$$

and therefore initially

$$
\rho_{\overline{\mathrm{K}}}(t=0)=\alpha \tilde{N}_{\overline{\mathrm{q}}} \bar{\rho}_{\mathrm{s}} s_{\mathrm{H}} / s_{\mathrm{Q}} .
$$

The entropy ratio factor $s_{\mathrm{H}} / s_{\mathrm{O}} \sim 1 / 10$ describes the dilution of strange particles in the transition from quark-gluon plasma to hadronic gas that was discussed following eq. (7.7). When the time-evolution of 
the fireball volume $V$ is given by appropriate hydrodynamical equations, or by other assumptions, e.g. as discussed in section 1.5 , eqs. (7.12), (7.19)-(7.21) and (7.23) fully determine the variation of strange particle densities with time.

\subsection{Strange particle abundances: Baryon-rich plasma}

We can now proceed to compute the strange particle abundances emerging from nuclear collisions. For $t \leq t_{0}$, eq. (7.20) must be used with the reaction mechanisms described in section 3 and given again in eq. (7.8). Throughout this section we assume that the initial plasma temperature $T_{0}=250 \mathrm{MeV}$, the plasma radius $R_{0}=3 \mathrm{fm}$, the plasma time $t_{0}$ being assumed as $1.5 R_{0}=4.5 \mathrm{fm} / c$. The strong coupling constant $\alpha_{\mathrm{s}}=0.6$ is taken and $m_{\mathrm{s}}=170 \mathrm{MeV}$ is employed in the plasma state. The table 7.1 presents the implicitly assumed pure plasma lifetimes $t_{\mathrm{c}}$ and time $t_{\mathrm{h}}$ at which plasma gas coexistence ceases (see fig. 1.5) resulting from assuming that the fireball volume grows with time as $V \sim t^{n}, n=1$ and 3 . The degree of saturation, $\rho_{\mathrm{s}} / \rho_{\mathrm{s}}^{\infty}$, of the strange quark phase space at $t=t_{\mathrm{c}}$ is also displayed. It is found from integration of eq. (7.20) up to $t=t_{\mathrm{c}}$ with the dilution term

$$
\frac{\mathrm{d}}{\mathrm{d} t} \ln V=\frac{n}{t} ; \quad t<t_{\mathrm{c}}
$$

We notice that the slow expansion, $n=1$, leads to very appreciable pure plasma lifetime of $17-32 \mathrm{fm} / \mathrm{c}$ depending somewhat on $\mu_{\mathbf{b}} / T$ and results in completely saturated strangeness phase space. The fast radial expansion, $n=3$, is more in agreement with naive expectations for the baryon-rich plasma of section 1.5 .1 - the lifetime of pure plasma state ranges from 7 to $9 \mathrm{fm} / c$ and the strangeness phase space is about $90 \%$ saturated when hadronization begins.

At $t=t_{\mathrm{c}}$ the plasma state commences to coexist with the hadronic gas. We turn now to our plasma fragmentation-recombination model, see section 4 . According to the scenario presented in the previous subsection, we further have

$$
V_{\mathbf{H}}(t)=V_{0}\left(t / t_{0}\right)^{n}\left[1-\left(t_{\mathrm{c}} / t\right)^{n}\right] r /(r-1)
$$

Table 7.1

Characteristic times and strangeness equilibration of hot hadronic matter in nuclear

\begin{tabular}{|c|c|c|c|c|c|c|c|}
\hline \multirow{2}{*}{$\begin{array}{l}\mu_{\mathrm{b}}^{\mathrm{cr}} \\
(\mathrm{MeV}) \\
\end{array}$} & \multirow[b]{2}{*}{$\mu_{h} / T$} & $\begin{array}{l}t_{\mathrm{c}}^{(1)} \\
(\mathrm{fm} / c)\end{array}$ & $\rho_{\mathrm{s}} / \rho_{\mathrm{s}}^{\infty}$ & $\begin{array}{l}t_{\mathrm{h}}^{(1)} \\
(\mathrm{fm} / \mathrm{c})\end{array}$ & $\begin{array}{l}t_{\mathrm{c}}^{(3)} \\
(\mathrm{fm} / \mathrm{c})\end{array}$ & $\rho_{\mathrm{s}} / \rho_{\mathrm{s}}^{\infty}$ & $\begin{array}{l}t_{\mathrm{h}}^{(3)} \\
(\mathrm{fm} / c)\end{array}$ \\
\hline & & \multicolumn{2}{|c|}{ cf. fig. 7.4} & \multicolumn{4}{|c|}{ cf. fig. 7.3} \\
\hline 0 & 0 & 17 & 1 & 162 & 7 & 0.5 & 14 \\
\hline 200 & 1.3 & 18 & 1 & 165 & 7 & 0.68 & 15 \\
\hline 400 & 2.7 & 22 & 1 & 173 & 8 & 0.90 & 15 \\
\hline 600 & 4.6 & 32 & 1 & 193 & 9 & 0.98 & 16 \\
\hline $\begin{array}{l}t_{\mathrm{c}}: \\
t_{\mathrm{h}}: \\
\rho_{\mathrm{s}} / \rho_{\mathrm{s}}^{\infty}: \\
(1)\end{array}$ & \multicolumn{7}{|c|}{$\begin{array}{l}\text { beginning of coexistence phase. } \\
\text { end of coexistence phase. } \\
\text { degree of strange quark saturation of plasma phase space at } t=t_{\mathrm{c}} \text {. } \\
V \sim t ; \quad \text { (3): } \quad V \sim t^{3} \text {. }\end{array}$} \\
\hline
\end{tabular}
collisions 


$$
V_{\mathrm{Q}}(t)=V_{0}\left(t / t_{0}\right)^{n}\left[\left(t_{\mathrm{c}} / t\right)^{n}-1 / r\right] r /(r-1)
$$

where $r$ is obtained from eq. (7.7):

$$
r=s_{\mathrm{Q}} / s_{\mathrm{H}} .
$$

Naturally we find, adding eqs. (7.25a) and (7.25b)

$$
V(t)=V_{\mathrm{H}}(t)+V_{\mathrm{Q}}(t)=V_{0}\left(t / t_{0}\right)^{n} .
$$

The functional forms shown in eqs. (7.25) are direct consequences of the requirement (7.26) and the assumptions made, such as eqs. (7.3) and (7.7). We further note that the end of the mixed phase is at $V_{\mathrm{Q}}=0$, i.e.

$$
t_{\mathrm{h}}=r^{1 / n} t_{\mathrm{c}} .
$$

The times $t_{\mathrm{h}}$ shown in table 7.1 arise from actual $r$ obtained inserting eqs. (7.5) and (7.6) into eq. (7.25c). The case $n=1$ does appear to exaggerate the lifetime of the mixed phase and $n=3$ may be a better choice for a 3-dimensional expansion, as it implies $R \sim t, T \sim 1 / t$.

Thus for $t_{\mathrm{c}}<t<t_{\mathrm{h}}$ while the volume evolves according to eqs. (7.25), the strange quark density evolves according to the undiluted eq. (7.12), since the entire dilution occurs through expansion of the hadronic gas part of the reaction volume due to the assumed exact correlation of quark-gluon plasma volume expansion with conversion into hadronic gas phase. However, we allow for temperature and chemical potential time dependence and $T \sim(1 / t)^{n / 3}$ particularly influences the reaction constant $R_{\mathrm{s}}$, cf. eq. (7.8) and eq. (3.13). The hadronic gas eq. (7.19) is used to describe the evolution of the various strange hadrons after the following generalization has been made

$$
\frac{\mathrm{d}}{\mathrm{d} t} \rho_{i}(t)=\sum_{n} R_{i}^{(n)}+\left(\rho_{i}^{\text {com }} \frac{s_{\mathrm{H}}}{s_{\mathrm{O}}}-\rho_{i}(t)\right) \frac{\mathrm{d}}{\mathrm{d} t}\left(\ln V_{\mathrm{H}}\right) .
$$

Here $\rho_{i}$ is the abundance of hadronic gas particles we seek, and $\rho_{i}^{\text {com }}$ are the combinatoric densities, e.g.:

$$
\rho_{\overline{\mathrm{K}}}^{\mathrm{com}}=\frac{\alpha^{*}}{2} \rho_{\overline{\mathrm{q}}} \rho_{\mathrm{s}}, \quad \rho_{\mathrm{Y}}^{\mathrm{com}}=\frac{\beta^{*}}{3 !} \rho_{\mathrm{q}}^{2} \rho_{\mathrm{s}},
$$

etc., compare eqs. (4.17) and (4.18). We have used volume reduced combinatoric factors

$$
\alpha^{*}=V_{\mathrm{Q}} \alpha, \quad \beta^{*}=V_{\mathrm{Q}}^{2} \beta
$$

such that eqs. (4.21), (4.22a) and (4.22b), for the $\alpha^{*}, \beta^{*}$, are given in terms of densities and not absolute abundances. Finally, $R_{i}^{(n)}$ is the reaction contribution of channel $n$ to the $i$ th particle considered as developed in section 5 and explicitly presented in appendix A.

As we have seen in section 4, the fragmentation mechanism in the quark-gluon plasma recombination model uses the surplus entropy of the plasma to generate additional partons. These additional 
partons contribute to the formation of hadrons, ensuring conservation or increase of entropy over the phase transition. In section 4 we considered both fragmentation of gluons and of quarks. For vanishing baryochemical potential $\mu_{\mathrm{b}}=0$ we found that some fragmentation of quarks is needed for the entropy balance, but considerably less than gluon fragmentation. When the consideration is extended to finite values of $\mu_{\mathrm{b}}$, it is found that quark fragmentation becomes less important, and gluon fragmentation alone is sufficient to conserve entropy [Ko86]. It is, therefore, not unwarranted to neglect quark fragmentation in an exploratory calculation of the expected particle abundances for different values of the baryochemical potential $\mu_{\mathrm{b}}$, and to take only gluon fragmentation into account.

\subsection{Numerical results}

Chemical evolution equation for the three expansion steps (quark-gluon plasma, phase transition, mixed phase) were first solved numerically by Kapusta and Mekjian [KM85]. Their analysis was only concerned with baryon-free systems, including pions and kaons in the hadronic gas phase. The interesting case of multistrange baryons, and strange antibaryons was not considered in this calculation. The conclusion of these authors that under almost all conditions the hadronic gas phase will emerge in absolute hadrochemical equilibrium must, therefore, be taken with some caution. Since the reaction $\pi \pi \rightarrow \mathrm{KK}$ has a relatively large cross section above threshold, it is not inconceivable that chemical equilibrium of kaons may be reached even in the absence of quark-gluon plasma formation. This would, however, require existence of a dense, hot hadronic fireball for a very long span of time or extremely

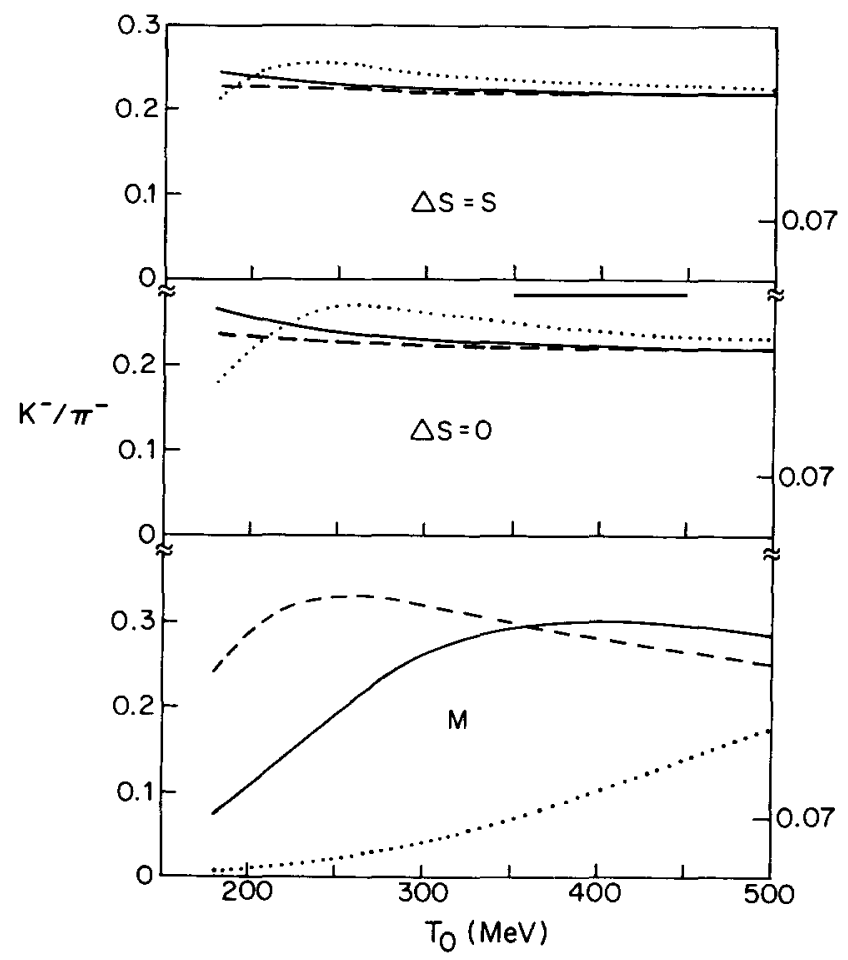

Fig. 7.2. $\mathrm{K}^{-} / \pi^{-}$ratio calculated by $[\mathrm{KM} 85]$ for a model involving pions and kaons only. M: mixed phase scenario; $\Delta S=0(\Delta S=S)$ supercooled plasma scenario without (with) entropy generation. The different curves correspond to different assumptions about parameters determining the reaction cross section $s$ (solid: 'most likely'; dashed: 'optimistic'; dotted: 'pessimistic'. See [KM85] for details). 
high temperatures $(T>200 \mathrm{MeV})$ in the hadronic phase. Whether such conditions are compatible with the kinetic properties of collision events, must be tested in future experiments.

Kapusta and Mekjian [KM85] also considered the case when no mixed phase is formed, and the phase transition is effected instead after strong supercooling of the plasma phase by a sudden transition to a superheated hadronic gas phase. As far as the results of kaon production are concerned, there is almost no difference compared with the mixed phase scenario in the final composition, because chemical equilibrium in a superheated hadronic phase of $300 \mathrm{MeV}$ temperature would be approached very quickly. Figure 7.2 shows the results of Kapusta and Mekjian [KM85] for the expected $\mathrm{K}^{-} / \pi^{-}$ ratio as a function of the initial temperature $T_{0}$ of the fireball. The upper two parts correspond to the scenario involving a supercooled plasma with $(\Delta S=S)$ or without $(\Delta S=0)$ entropy production in the transition, while the lower part represents the mixed phase scenario.

Let us now come to the results of calculations based on the full set of rate equations in the hadronic phase discussed in section 5, involving baryons and antibaryons in addition to pions and kaons [Ko86]. These calculations are based on the following picture of the evolution of the fireball: initially a quark-gluon plasma is formed, which expands and cools until it reaches at $t=t_{\mathrm{c}}$ the phase transition point towards the hadronic gas phase. Temperature and baryochemical potential are then kept constant and the plasma is gradually converted into hadronic gas. During this process the volume of the mixed
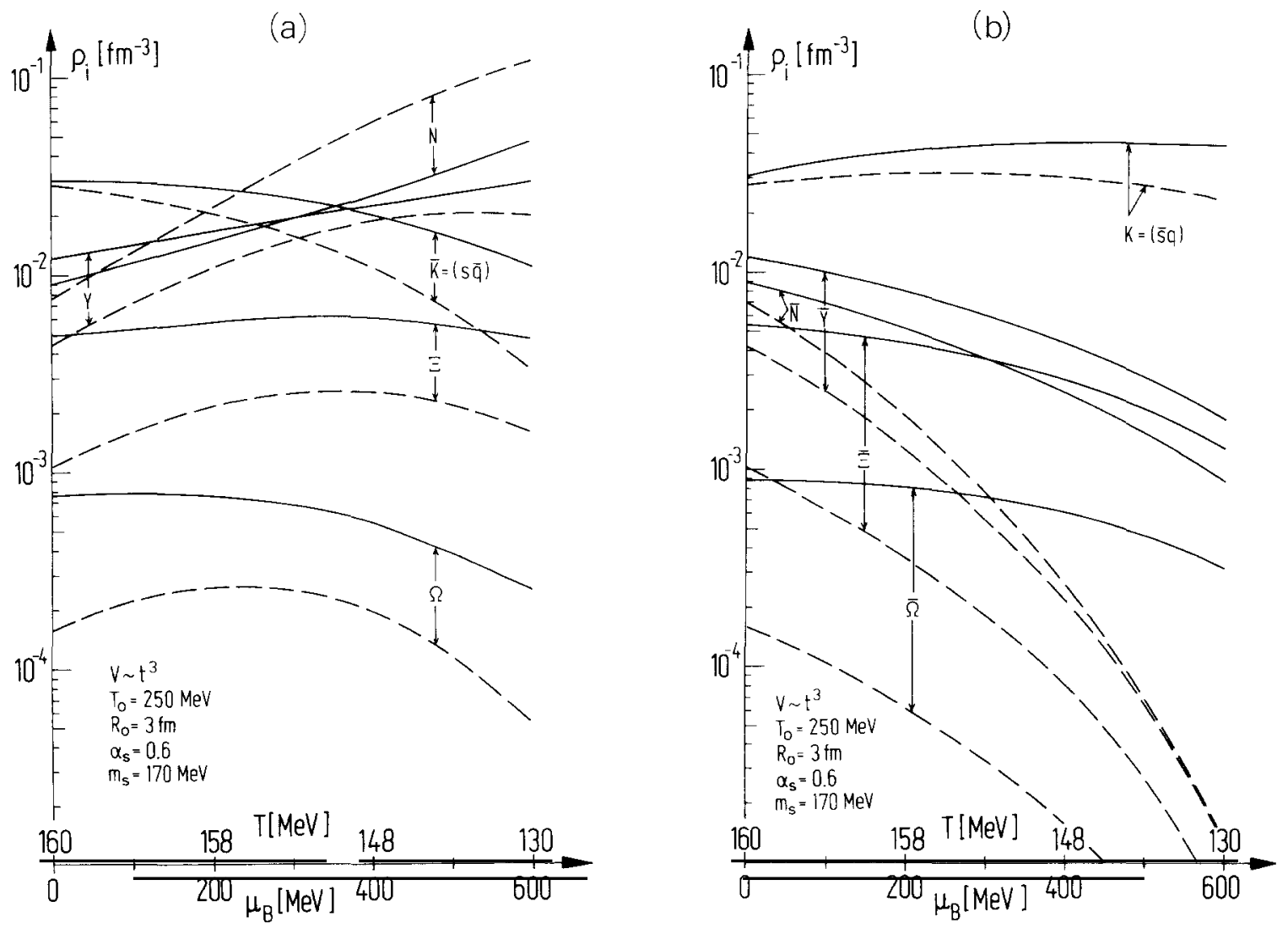

Fig. 7.3. Strange particle abundances assuming fast volume expansion $(V \sim t)$ and recombination with gluon fragmentation: (a) s-quark and (b) $\bar{s}$-quark carrying hadrons at the end of coexistence of quark gluon plasma and hadronic gas phase, as a function of critical values of $T$ and $\mu_{\mathrm{b}}$. Dashed: equilibrium abundances in the hadronic gas phase at the same values of $T$ and $\mu_{\mathrm{b}}$. 
phase grows according to the requirement of entropy conservation as expressed in eqs. (7.25). The calculation is stopped at $t=t_{\mathrm{h}}$ when the quark-gluon plasma is fully converted into hadrons. In the first part of the expansion, when only the plasma phase is present, eq. (7.20), for strange quark production is solved. Later, when plasma and hadronic phase coexist, eq. (7.12) is solved for strange quark density in the plasma phase (light quarks and gluons are always assumed to be in full equilibrium), while eqs. (7.28) are solved for the evolution of abundances of $\pi, \mathrm{K}, \mathrm{N}, \mathrm{Y}, \Xi, \Omega$ and antiparticles in the hadronic phase. In the hadronization process, mesons and baryons are assumed to form according to the fragmentation-recombination scenario expressed by eqs. (7.29) and presented in detail in section 4. Gluon fragmentation ensures approximate entropy conservation by providing the additional partons required to fill the larger hadronic gas volume with mesons and baryons.

First, consider the particle abundances presented in figs. 7.3 and 7.4. Both figures differ in the choice of the exponent $n$ in eq. (7.24) and correspond to fast $(n=3)$ resp. slow $(n=1)$ volume expansion. Parts (a) and (b) in each case refer to abundances of hadrons containing s- and $\bar{s}$-quarks, respectively. The abundances are presented as found at $t=t_{\mathrm{h}}$, the time when the plasma phase has been completely converted and the hadronic gas phase is quite thin already. The curves are given as function of the values of the thermodynamical variables $T$ and $\mu_{\mathrm{b}}$ at the point where the critical curve is crossed. Throughout the calculation a constant $T / \mu_{\mathrm{b}}$ ratio is assumed. The dashed curves are the equilibrium hadronic gas results as obtained according to the grand canonical approach, section 6.1 .
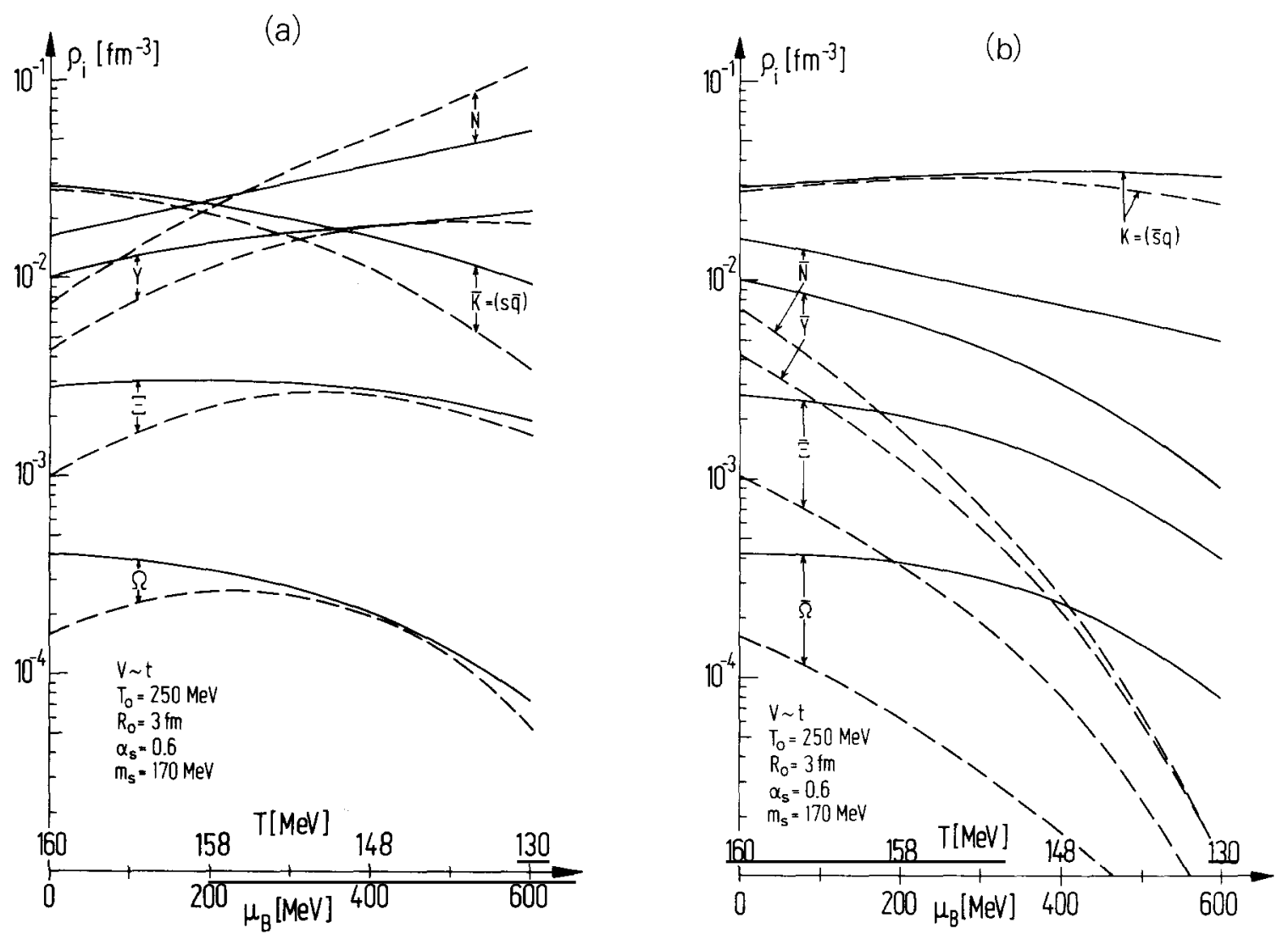

Fig. 7.4. Strange particle abundances assuming slow (longitudinal) volume expansion $(V \sim t)$ and recombination with gluon fragmentation, else same as fig. 7.3. 
As discussed in section 5, the actual abundances of strange hadrons in the collisions in which no plasma is formed would be significantly smaller, in particular, the strange antibaryons would be far from their hadronic gas equilibrium values. As we see from the presented results, there is a very significant overabundance of strange antibaryons found, which - incidentally - does not rely on the mechanism of quark-gluon fragmentation (but fragmentation increases overall), whether other hadrons come out above or just at their chemical equilibrium abundance depends to a large extent on the lifetime of the mixed phase. For short lifetime (fig. 7.3) considerable overabundance of hyperons $Y=(\Lambda, \Sigma)$ and cascade particles $\Xi$ is predicted. As could be expected from naive arguments concerning $s-\overline{\mathrm{s}}$ symmetry in plasma, the abundance of $\Omega$ and $\bar{\Omega}$ turn out to be quite similar, independent of the value of $\mu_{\mathrm{b}}$.

For reasons of experimental practicability it is important that the densities of strange antibaryons $\bar{Y}$, $\bar{\Xi}$ are in the range $10^{-3}$ to $10^{-2} / \mathrm{fm}^{3}$ at the time of break up. Given the large fireball volume of several thousand $\mathrm{fm}^{3}$ at this time, a number of these particles are expected to be produced in each single reaction event leading to formation of quark-gluon plasma. This fact greatly reduces the danger of mistaking rare fluctuations in purely hadronic reaction events for plasma formation, and allows for accumulation of high-statistics data.

By contrast, the proposal of Heinz, Subramanian and Greiner [HSG84, He85] to utilize antinuclei as signature for quark-gluon plasma is plagued by extremely small abundances of these particles, e.g. antideuterons are predicted to occur with abundance $10^{-6}$ to $10^{-4} / \mathrm{fm}^{3}$, and anti-alpha particles only with a density of $10^{-12}$ to $10^{-3} / \mathrm{fm}^{3}$. Observation of a very large number of quark-gluon plasma events and great trust in the absence of substantial rare fluctuations away from hadronic equilibrium would be required to establish the presence of quark-gluon plasma from such a signal. (The particular deficiencies of the calculation of Heinz et al. [He85], e.g. violation of entropy conservation during the hadronization process, has been discussed before, see section 4.4.)

In conclusion, our calculations presented above confirm the particular suitability of strange antibaryons for diagnosis and study of the quark-gluon plasma state. However, they should not yet be taken as the ultimate picture on expected particle abundances, as more realistic plasma evolution and dissociation models have to be developed. In particular, the combination of the hadrochemical rate equations with a state-of-the-art solution of the relativistic hydrodynamical evolution equations including a phase transition would be a worthwhile next step.

\section{Highlights}

The results collected in this report, some of them shown for the first time, substantiate the expectation that abundances of strange particles, most notably of strange antibaryons, provide a powerful tool to probe the quark-gluon plasma in nuclear reactions at very high energy. We will not try here to comprehensively repeat the discussions set forth in the various preceding sections, but instead concentrate on the results that highlight the unique features of strange particle production.

We begin recalling the results obtained in section 4 where we have shown that in case of equilibrated strangeness abundances in different phases of hadronic matter, strange hadronic particles are predicted to emerge from the quark-gluon plasma phase more abundantly than this would be the case in a purely hadronic gas (figs. 4.3 and 4.4). To a large degree this behaviour is a consequence of numerous fragmentation processes required in the break-up of the plasma phase in order to obey the 2nd law of thermodynamics and also due to the fact that $\langle\mathrm{s}\rangle /\langle\mathrm{q}\rangle$ relative abundance is enhanced. It is important here to emphasise that the specific example of strong enhancement in the strange antihyperon $\overline{\mathrm{Y}}$ 
production was found to persist for all hadronization scenarios (see fig. 4.4). The enhancement of $s \bar{s}[\phi]$, fig. 4.3, appears to be less pronounced. On the other hand, for the more common strangeness carrying particles, viz. kaons and hyperons, no impressive but nonetheless also an observable surplus as compared to the hadronic equilibrium abundances is expected. However, for these particles, a failure to find above-equilibrium abundance cannot be taken as a signal for the absence of a plasma phase. In other words: $\bar{Y}$ abundance anomalies (and also $\bar{\Xi}$ and $\bar{\Omega}$ ) are characteristic for plasma formation because they will exceed the size of the phase space of individual hadrons, while $K, \bar{K}$ and $Y$ (as well as $\pi$ ) anomalies will be there, but are not considered to be characteristic because their abundances will be near the phase space limit.

Another important point we emphasise here again is that hadronic reaction rates are much too small to allow for equilibration of strange particle densities during a nuclear reaction unless plasma is formed. After a reaction time of $10 \mathrm{fm} / c$ (about $3 \times 10^{-23} \mathrm{~s}$ ) the gross density of strange particles would still remain an order of magnitude off its equilibrium value (see fig. 5.4). This lack of saturation of strange particle phase space in hadronic gas has first been discussed and is well known for nuclear collisions in the energy range around $1 \mathrm{GeV} / \mathrm{u}$. For strange antibaryons the underpopulation is even more pronounced, with two to three orders of magnitude missing to equilibrium (fig. 5.5). This means that even the observation of saturated hadronic equilibrium for these particles would be a mystery, pointing to the temporary presence of a quark-gluon plasma seed, at least. The predicted antihyperon phase space overabundance, resulting from the quark-gluon plasma region, can - if actually observed - only be explained by invoking the deconfined plasma state. These results are confirmed by the dynamical evolution scenarios developed in section 7: even when all surplus plasma entropy is used for expansion rather than quark-gluon fragmentation, the overabundance of strange antibaryons persists at least until the phase transition is completed, cf. figs. 7.3 and 7.4 (fast and slow phase transition scenarios, respectively).

The usefulness of strange antibaryons as signals for quark-gluon plasma formation is most strikingly emphasized by a comparison of fig. 5.5 and fig. 7.3. To wit, fig. 7.3 showed the results of a calculation along the lines of a 'fast' expansion scenario, where a quark-gluon plasma was assumed to be formed in the collision, cooling to the critical temperature at about $8 \mathrm{fm} / c$ after the start of the reaction, and completing the break-up into hadrons within an additional time interval of $7 \mathrm{fm} / c$. Altogether, the high-density stage of the reaction lasts for about $5 \times 10^{-23} \mathrm{~s}$, after which time the densities of strange antibaryons are predicted to exceed substantially the hadronic equilibrium values. In absence of a quark-gluon plasma state, the abundance of strange baryons and antibaryons would still be far below saturation after this reaction time, as fig. 5.5 shows, by factors ranging from 20 for antihyperons $\Lambda, \Sigma$ to more than 100 for triple strange baryons $\Omega, \bar{\Omega}$.

The source of all these results can be traced back to the fact that strange quark-pair production in the plasma phase proceeds at a sufficiently fast rate to permit statistical equilibrium abundance to be established in less than $10 \mathrm{fm} / c$. As discussed in section 3, this is mainly due to the abundant presence of gluonic excitations, allowing for quark-pair production in gluon-gluon collisions. In a sense, therefore, abundant strange antibaryon production is indicative of an environment in which two-gluon processes dominate over single-gluon exchange phenomena.

In conclusion, we have shown on the basis of a dynamical theoretical model of strange particle production in nuclear collisions that normally rare strange antibaryon $S$ or particles with multiple strangeness content (antibaryons $\bar{\Lambda}, \bar{\Sigma}, \bar{\Xi}, \bar{\Omega}$ ) provide a very promising experimental signal in the search of the quark-gluon plasma. In particular, abundant strangeness production is indicative of the presence of gluon excitations, a characteristic property of the deconfined QCD phase. 


\section{Acknowledgements}

One of us (J.R.) would like to thank R. Hagedorn and M. Danos for continuous support and fruitful discussions during the five year research period which contributed to the here presented results. He also thanks the Theory Division, CERN, for frequent and kind hospitality during the course of which some of the material presented here has been developed. One of us (B.M.) acknowledges the hospitality of the Institute for Nuclear Study of the University of Tokyo, and support by the Japanese Minister of Education, while portions of this report were completed.

A number of colleagues made constructive comments concerning the first version of the manuscript. In particular, we would like to thank, in that respect, J. Cleymans and M. Soyeur. We also acknowledge many useful discussions with R. Bock, C. Gruhn, H. Gutbrod, G. London, S. Nagamiya, H. Pugh, E. Quercigh, A. Shor, R. Stock, H.J. Specht and W. Willis, concerning the experimental programs at CERN and BNL.

Finally, we thank Mrs. Joan Parsons for her excellent and faultless typing of the manuscript and patience with our numerous revisions and additions.

\section{Appendix A: Master equations for strangeness evolution in hadronic gas}

The set of differential equations considered here reads:

$$
\begin{aligned}
\frac{\mathrm{d}}{\mathrm{d} t} \rho_{\Omega}= & \left\langle\sigma_{\pi \mathrm{N}}^{\mathrm{KY}} v_{\pi \mathrm{N}}\right\rangle\left\{P_{2}\left[\rho_{\pi} \rho_{\Xi}-R_{1} \rho_{\mathrm{K}} \rho_{\Omega}\right]\right\}+\left\langle\sigma_{\overline{\mathrm{K} N}}^{\pi \mathrm{Y}} v_{\overline{\mathrm{K} N}}\right\rangle\left\{A_{2}\left[\rho_{\overline{\mathrm{K}}} \rho_{\Xi}-R_{5} \rho_{\Omega} \rho_{\pi}\right]\right\} \\
& -\left\langle\sigma_{\mathrm{N} \overline{\mathrm{N}}}^{5 \pi} v_{\mathrm{NN}}\right\rangle D_{3}\left\{\rho_{\Omega} \rho_{\overline{\mathrm{N}}}-R_{11} \rho_{\overline{\mathrm{K}}}^{3} \rho_{\pi}^{2}\right\} \\
\frac{\mathrm{d}}{\mathrm{d} t} \rho_{\bar{\Omega}}= & \left\langle\sigma_{\pi \mathrm{N}}^{\mathrm{KY}} v_{\pi \mathrm{N}}\right\rangle\left\{P_{2}\left[\rho_{\pi} \rho_{\Xi}-R_{1} \rho_{\overline{\mathrm{K}}} \rho_{\bar{\Omega}}\right]\right\}+\left\langle\sigma_{\overline{\mathrm{K} N}}^{\pi \mathrm{Y}} v_{\overline{\mathrm{K}} \mathrm{N}}\right\rangle\left\{A_{2}\left[\rho_{\mathrm{K}} \rho_{\Xi}-R_{5} \rho_{\bar{\Omega}} \rho_{\pi}\right]\right\} \\
& -\left\langle\sigma_{\mathrm{N} \overline{\mathrm{N}}}^{5 \pi} v_{\mathrm{NN}}\right\rangle\left\{D_{3}\left[\rho_{\bar{\Omega}} \rho_{\mathrm{N}}-R_{11} \rho_{\mathrm{K}}^{3} \rho_{\pi}^{2}\right]\right\} \\
\frac{\mathrm{d}}{\mathrm{d} t} \rho_{\Xi}= & \left\langle\sigma_{\pi \mathrm{N}}^{\mathrm{KY}} v_{\pi \mathrm{N}}\right\rangle\left\{P_{1}\left(\rho_{\mathrm{Y}} \rho_{\pi}-R_{2} \rho_{\Xi} \rho_{\mathrm{K}}\right)-P_{2}\left(\rho_{\pi} \rho_{\Xi}-R_{1} \rho_{\Omega} \rho_{\mathrm{K}}\right)\right\} \\
& +\left\langle\sigma_{\overline{\mathrm{K} N}}^{\pi \mathrm{Y}} v_{\overline{\mathrm{K}} \mathrm{N}}\right\rangle\left\{A_{1}\left(\rho_{\overline{\mathrm{K}}} \rho_{\mathrm{Y}}-R_{6} \rho_{\Xi} \rho_{\pi}\right)-A_{2}\left(\rho_{\overline{\mathrm{K}}} \rho_{\Xi}-R_{5} \rho_{\Omega} \rho_{\pi}\right)\right\} \\
& -\left\langle\sigma_{\mathrm{NN}}^{5 \pi} v_{\mathrm{NN}}\right\rangle\left\{D_{2}\left[\rho_{\Xi} \rho_{\overline{\mathrm{N}}}-R_{10} \rho_{\mathrm{K}}^{2} \rho_{\pi}^{3}\right]\right\} \\
\frac{\mathrm{d}}{\mathrm{d} t} \rho_{\bar{\Xi}}= & \left\langle\sigma_{\pi \mathrm{N}}^{\mathrm{KY}} v_{\pi \mathrm{N}}\right\rangle\left\{P_{1}\left(\rho_{\overline{\mathrm{Y}}} \rho_{\pi}-R_{2} \rho_{\bar{\Xi}} \rho_{\overline{\mathrm{K}}}\right)-P_{2}\left(\rho_{\pi} \rho_{\bar{\Xi}}-R_{1} \rho_{\bar{\Omega}} \rho_{\overline{\mathrm{K}}}\right)\right\} \\
& +\left\langle\sigma_{\overline{\mathrm{K}} \mathrm{N}}^{\pi \mathrm{Y}} v_{\overline{\mathrm{K}} \mathrm{N}}\right\rangle\left\{A_{1}\left(\rho_{\mathrm{K}} \rho_{\overline{\mathrm{Y}}}-R_{6} \rho_{\Xi} \rho_{\pi}\right)-A_{2}\left(\rho_{\mathrm{K}} \rho_{\bar{\Xi}}-R_{5} \rho_{\bar{\Omega}} \rho_{\pi}\right)\right\} \\
& -\left\langle\sigma_{\mathrm{N} \overline{\mathrm{N}}}^{5 \pi} v_{\mathrm{N} \overline{\mathrm{N}}}\right\rangle\left\{D_{2}\left[\rho_{\Xi} \rho_{\mathrm{N}}-R_{10} \rho_{\mathrm{K}}^{2} \rho_{\pi}^{3}\right]\right\},
\end{aligned}
$$




$$
\begin{aligned}
\frac{\mathrm{d}}{\mathrm{d} t} \rho_{\mathrm{Y}}= & \left\langle\sigma_{\pi \mathrm{N}}^{\mathrm{KY} v_{\pi \mathrm{N}}}\right\rangle\left\{\rho_{\pi} \rho_{\mathrm{N}}-R_{3} \rho_{\mathrm{Y}} \rho_{\mathrm{K}}-P_{1}\left(\rho_{\pi} \rho_{\mathrm{Y}}-R_{2} \rho_{\mathrm{K}} \rho_{\Xi}\right)\right\} \\
& +\left\langle\sigma_{\overline{\mathrm{K} N}}^{\pi \mathrm{Y}} v_{\overline{\mathrm{K}} \mathrm{N}}\right\rangle\left\{\rho_{\overline{\mathrm{K}}} \rho_{\mathrm{N}}-R_{7} \rho_{\pi} \rho_{\mathrm{Y}}-A_{1}\left(\rho_{\overline{\mathrm{K}}} \rho_{\mathrm{Y}}-R_{6} \rho_{\pi} \rho_{\Xi}\right)\right\} \\
& -\left\langle\sigma_{\mathrm{N} \overline{\mathrm{N}}}^{5 \pi} v_{\mathrm{N} \overline{\mathrm{N}}}\right\rangle\left\{D_{1}\left[\rho_{\mathrm{Y}} \rho_{\overline{\mathrm{N}}}-R_{9} \rho_{\overline{\mathrm{K}}} \rho_{\pi}^{4}\right]\right\}
\end{aligned}
$$

$$
\begin{aligned}
\frac{\mathrm{d}}{\mathrm{d} t} \rho_{\overline{\mathrm{Y}}}= & \left\langle\sigma_{\pi \mathrm{N}}^{\mathrm{KY}} v_{\pi \mathrm{N}}\right\rangle\left\{\rho_{\pi} \rho_{\overline{\mathrm{N}}}-R_{3} \rho_{\overline{\mathrm{Y}}} \rho_{\overline{\mathrm{K}}}-P_{1}\left(\rho_{\pi} \rho_{\overline{\mathrm{N}}}-R_{2} \rho_{\overline{\mathrm{K}}} \rho_{\Xi}\right)\right\} \\
& +\left\langle\sigma_{\overline{\mathrm{K} N}}^{\pi \mathrm{Y}} v_{\overline{\mathrm{K} N}}\right\rangle\left\{\rho_{\mathrm{K}} \rho_{\overline{\mathrm{N}}}-R_{7} \rho_{\pi} \rho_{\overline{\mathrm{Y}}}-A_{1}\left(\rho_{\mathrm{K}} \rho_{\overline{\mathrm{Y}}}-R_{6} \rho_{\pi} \rho_{\Xi}\right)\right\} \\
& -\left\langle\sigma_{\mathrm{NN}}^{5 \pi} v_{\mathrm{NN}}\right\rangle\left\{D_{1}\left[\rho_{\overline{\mathrm{Y}}} \rho_{\mathrm{N}}-R_{9} \rho_{\mathrm{K}} \rho_{\pi}^{4}\right]\right\}
\end{aligned}
$$

$$
\begin{aligned}
\frac{\mathrm{d}}{\mathrm{d} t} \rho_{\mathrm{N}}= & -\left\langle\sigma_{\pi \mathrm{N}}^{\mathrm{KY}} v_{\pi \mathrm{N}}\right\rangle\left[\rho_{\pi} \rho_{\mathrm{N}}-R_{3} \rho_{\mathrm{K}} \rho_{\mathrm{Y}}\right]-\left\langle\sigma_{\overline{\mathrm{K}} \mathrm{N}}^{\mathrm{Y} \pi} v_{\overline{\mathrm{K} N}}\right\rangle\left[\rho_{\overline{\mathrm{K}}} \rho_{\mathrm{N}}-R_{7} \rho_{\pi} \rho_{\mathrm{Y}}\right] \\
& -\left\langle\sigma_{\mathrm{N} \overline{\mathrm{N}}}^{5 \pi} v_{\mathrm{N} \overline{\mathrm{N}}}\right\rangle\left\{\rho_{\mathrm{N}} \rho_{\overline{\mathrm{N}}}-R_{8} \rho_{\pi}^{5}+D_{1}\left(\rho_{\mathrm{N}} \rho_{\overline{\mathrm{Y}}}-R_{9} \rho_{\mathrm{K}} \rho_{\pi}^{4}\right)\right. \\
& \left.+D_{2}\left(\rho_{\mathrm{N}} \rho_{\Xi}-R_{10} \rho_{\mathrm{K}}^{2} \rho_{\pi}^{3}\right)+D_{3}\left(\rho_{\mathrm{N}} \rho_{\bar{\Omega}}-R_{11} \rho_{\mathrm{K}}^{3} \rho_{\pi}^{2}\right)\right\}
\end{aligned}
$$$$
\frac{\mathrm{d}}{\mathrm{d} t} \rho_{\overline{\mathrm{N}}}=-\left\langle\sigma_{\pi \mathrm{N}}^{\mathrm{KY}} v_{\pi \mathrm{N}}\right\rangle\left[\rho_{\pi} \rho_{\overline{\mathrm{N}}}-R_{1} \rho_{\overline{\mathrm{K}}} \rho_{\overline{\mathrm{Y}}}\right]-\left\langle\sigma_{\overline{\mathrm{K}} \mathrm{N}}^{\mathrm{Y} \pi} v_{\overline{\mathrm{K} N}}\right\rangle\left[\rho_{\mathrm{K}} \rho_{\overline{\mathrm{N}}}-R_{7} \rho_{\pi} \rho_{\overline{\mathrm{Y}}}\right]
$$$$
-\left\langle\sigma_{\mathrm{N} \tilde{\mathrm{N}}}^{5 \pi} v_{\mathrm{NN}}\right\rangle\left\{\rho_{\mathrm{N}} \rho_{\overline{\mathrm{N}}}-R_{8} \rho_{\pi}^{5}+D_{1}\left(\rho_{\overline{\mathrm{N}}} \rho_{\mathrm{Y}}-R_{9} \rho_{\overline{\mathrm{K}}} \rho_{\pi}^{4}\right)\right.
$$$$
\left.+D_{2}\left(\rho_{\overline{\mathrm{N}}} \rho_{\Xi}-R_{10} \rho_{\overline{\mathrm{K}}}^{2} \rho_{\pi}^{3}\right)+D_{3}\left(\rho_{\overline{\mathrm{N}}} \rho_{\Omega}-R_{11} \rho_{\overline{\mathrm{K}}}^{3} \rho_{\pi}^{2}\right)\right\},
$$

$$
\begin{aligned}
\frac{\mathrm{d}}{\mathrm{d} t} \rho_{\overline{\mathrm{K}}}= & \left\langle\sigma_{\pi \mathrm{N}}^{\mathrm{KY}} v_{\pi \mathrm{N}}\right\rangle\left\{\rho_{\pi} \rho_{\overline{\mathrm{N}}}-R_{3} \rho_{\overline{\mathrm{K}}} \rho_{\overline{\mathrm{Y}}}+P_{1}\left(\rho_{\pi}^{2} \rho_{\overline{\mathrm{Y}}}-R_{2} \rho_{\overline{\mathrm{K}}} \rho_{\bar{\Xi}}\right)\right. \\
& \left.+P_{2}\left(\rho_{\pi} \rho_{\bar{\Xi}}-R_{1} \rho_{\overline{\mathrm{K}}} \rho_{\bar{\Omega}}\right)+P_{3}\left(\rho_{\pi}^{2}-R_{4} \rho_{\mathrm{K}} \rho_{\overline{\mathrm{K}}}\right)\right\} \\
& -\left\langle\sigma_{\overline{\mathrm{K} N}}^{\pi \mathrm{Y}} v_{\overline{\mathrm{K} N}}\right\rangle\left\{\rho_{\overline{\mathrm{K}}} \rho_{\mathrm{N}}-R_{7} \rho_{\pi} \rho_{\mathrm{Y}}+A_{1}\left(\rho_{\overline{\mathrm{K}}} \rho_{\mathrm{Y}}-R_{6} \rho_{\pi} \rho_{\Xi}\right)+A_{2}\left(\rho_{\overline{\mathrm{K}}} \rho_{\Xi}-R_{5} \rho_{\pi} \rho_{\Omega}\right)\right\} \\
& +\left\langle\sigma_{\mathrm{NN}}^{5 \pi} v_{\mathrm{NN}}\right)\left\{D_{1}\left(\rho_{\overline{\mathrm{N}}} \rho_{\mathrm{Y}}-R_{9} \rho_{\overline{\mathrm{K}}} \rho_{\pi}^{4}\right)+D_{2}\left(\rho_{\overline{\mathrm{N}}} \rho_{\Xi}-R_{10} \rho_{\overline{\mathrm{K}}}^{2} \rho_{\pi}^{3}\right)+D_{3}\left(\rho_{\overline{\mathrm{N}}} \rho_{\Omega}-R_{11} \rho_{\overline{\mathrm{K}}}^{3} \rho_{\pi}^{2}\right)\right\},
\end{aligned}
$$

$$
\begin{aligned}
\frac{\mathrm{d}}{\mathrm{d} t} \rho_{\mathrm{K}}= & \left\langle\sigma_{\pi \mathrm{N}}^{\mathrm{KY}} v_{\pi \mathrm{N}}\right\rangle\left\{\rho_{\pi} \rho_{\mathrm{N}}-R_{3} \rho_{\mathrm{K}} \rho_{\mathrm{Y}}+P_{1}\left(\rho_{\pi} \rho_{\mathrm{Y}}-R_{2} \rho_{\mathrm{K}} \rho_{\Xi}\right)\right. \\
& \left.+P_{2}\left(\rho_{\pi} \rho_{\Xi}-R_{1} \rho_{\mathrm{K}} \rho_{\Omega}\right)+P_{3}\left(\rho_{\pi}^{2}-R_{4} \rho_{\overline{\mathrm{K}}} \rho_{\mathrm{K}}\right)\right\} \\
& -\left\langle\sigma_{\overline{\mathrm{K} N}}^{\pi \mathrm{Y}} v_{\overline{\mathrm{K}} \mathrm{N}}\right\rangle\left\{\rho_{\mathrm{K}} \rho_{\overline{\mathrm{N}}}-R_{7} \rho_{\pi} \rho_{\overline{\mathrm{Y}}}+A_{1}\left(\rho_{\mathrm{K}} \rho_{\overline{\mathrm{Y}}}-R_{6} \rho_{\pi} \rho_{\bar{\Xi}}\right)+A_{2}\left(\rho_{\mathrm{K}} \rho_{\bar{\Xi}}-R_{5} \rho_{\pi} \rho_{\bar{\Xi}}\right)\right\} \\
& +\left\langle\sigma_{\mathrm{N} \overline{\mathrm{N}}}^{5 \pi} v_{\mathrm{N} \overline{\mathrm{N}}}\right)\left\{D_{1}\left(\rho_{\mathrm{N}} \rho_{\overline{\mathrm{Y}}}-R_{9} \rho_{\mathrm{K}} \rho_{\pi}^{5}\right)+D_{2}\left(\rho_{\mathrm{N}} \rho_{\bar{\Xi}}-R_{10} \rho_{\mathrm{K}}^{2} \rho_{\pi}^{3}\right)+D_{3}\left(\rho_{\mathrm{N}} \rho_{\bar{\Omega}}-R_{11} \rho_{\mathrm{K}}^{3} \rho_{\pi}^{2}\right)\right\},
\end{aligned}
$$




$$
\begin{aligned}
\frac{\mathrm{d}}{\mathrm{d} t} \rho_{\pi}= & \left\langle\sigma_{\pi \mathrm{N}}^{\mathrm{KY}} v_{\pi \mathrm{N}}\right\rangle\left\{\left(\rho_{\pi} \rho_{\mathrm{N}}+\rho_{\pi} \rho_{\overline{\mathrm{N}}}\right)-R_{3}\left(\rho_{\mathrm{K}} \rho_{\mathrm{Y}}+\rho_{\overline{\mathrm{K}}} \rho_{\overline{\mathrm{Y}}}\right)\right. \\
& +P_{1}\left[\left(\rho_{\pi} \rho_{\mathrm{Y}}+\rho_{\pi} \rho_{\overline{\mathrm{Y}}}\right)-R_{2}\left(\rho_{\mathrm{K}} \rho_{\Xi}+\rho_{\overline{\mathrm{K}}} \rho_{\bar{\Xi}}\right)\right] \\
& \left.+P_{2}\left[\left(\rho_{\pi} \rho_{\Xi}+\rho_{\pi} \rho_{\bar{\Xi}}\right)-R_{1}\left(\rho_{\mathrm{K}} \rho_{\Omega}+\rho_{\overline{\mathrm{K}}} \rho_{\bar{\Omega}}\right)\right]+P_{3}\left[\rho_{\pi}^{2}-R_{4} \rho_{\mathrm{K}} \rho_{\overline{\mathrm{K}}}\right]\right\} \\
& +\left\langle\rho_{\overline{\mathrm{K} N}}^{\pi \mathrm{Y}} v_{\overline{\mathrm{K} N}}\right\rangle\left\{\left(\rho_{\overline{\mathrm{K}}} \rho_{\mathrm{N}}+\rho_{\mathrm{K}} \rho_{\overline{\mathrm{N}}}\right)-R_{7}\left(\rho_{\pi} \rho_{\mathrm{Y}}+\rho_{\pi} \rho_{\overline{\mathrm{Y}}}\right)\right. \\
& \left.+A_{1}\left[\left(\rho_{\overline{\mathrm{K}}} \rho_{\mathrm{Y}}+\rho_{\mathrm{K}} \rho_{\overline{\mathrm{Y}}}\right)-R_{5}\left(\rho_{\pi} \rho_{\Xi}+\rho_{\pi} \rho_{\bar{\Xi}}\right)\right]+A_{2}\left[\left(\rho_{\overline{\mathrm{K}}} \rho_{\Xi}+\rho_{\mathrm{K}} \rho_{\Xi}\right)-R_{5}\left(\rho_{\pi} \rho_{\Omega}+\rho_{\pi} \rho_{\bar{\Omega}}\right)\right]\right\} \\
& +\left\langle\rho_{\mathrm{N} \overline{\mathrm{N}}}^{5 \pi} v_{\mathrm{N} \overline{\mathrm{N}}}\right\rangle\left\{\rho_{\mathrm{N}} \rho_{\overline{\mathrm{N}}}-R_{8} \rho_{\pi}^{5}+D_{1}\left[\rho_{\mathrm{N}} \rho_{\overline{\mathrm{Y}}}+\rho_{\mathrm{Y}} \rho_{\overline{\mathrm{N}}}-R_{9}\left(\rho_{\overline{\mathrm{K}}} \rho_{\pi}^{4}+\rho_{\mathrm{K}} \rho_{\pi}^{4}\right)\right]\right. \\
& \left.+D_{2}\left[\rho_{\mathrm{N}} \rho_{\bar{\Xi}}+\rho_{\overline{\mathrm{N}}} \rho_{\Xi}-R_{10}\left(\rho_{\overline{\mathrm{K}}}^{2} \rho_{\pi}^{3}+\rho_{\mathrm{K}}^{2} \rho_{\pi}^{3}\right)\right]+D_{3}\left[\rho_{\mathrm{N}} \rho_{\bar{\Omega}}+\rho_{\overline{\mathrm{N}}} \rho_{\Omega}-R_{11}\left(\rho_{\overline{\mathrm{K}}}^{3} \rho_{\pi}^{2}+\rho_{\mathrm{K}}^{3} \rho_{\pi}^{2}\right)\right]\right\},
\end{aligned}
$$

with the following definitions

$$
\begin{aligned}
& P_{1}:=\frac{\left\langle\sigma_{\pi \mathrm{Y}} v_{\pi \mathrm{Y}}\right\rangle}{\left\langle\sigma_{\pi \mathrm{N}}^{\mathrm{KY}} v_{\pi \mathrm{N}}\right\rangle} ; \quad P_{2}:=\frac{\left\langle\sigma_{\pi \Xi}^{\mathrm{K} \Omega} v_{\pi \Xi}\right\rangle}{\left\langle\sigma_{\pi \mathrm{N}}^{\mathrm{KY}} v_{\pi \mathrm{N}}\right\rangle} ; \quad P_{3}:=\frac{\left\langle\sigma_{\pi \pi}^{\mathrm{KK}} v_{\pi \pi}\right\rangle}{\left\langle\sigma_{\pi \mathrm{N}}^{\mathrm{KY}} v_{\pi \mathrm{N}}\right\rangle} \\
& A_{1}:=\frac{\left\langle\sigma_{\mathrm{KY}}^{\pi \Xi} v_{\overline{\mathrm{K}} \mathrm{Y}}\right\rangle}{\left\langle\sigma_{\overline{\mathrm{K}} \mathrm{N}}^{\pi \mathrm{Y}} v_{\overline{\mathrm{K}} \mathrm{N}}\right\rangle} ; \quad A_{2}:=\frac{\left\langle\sigma_{\overline{\mathrm{K}} \Xi}^{\pi \Omega} v_{\overline{\mathrm{K}} \Xi}\right\rangle}{\left\langle\sigma_{\overline{\mathrm{K}} \mathrm{N}}^{\pi \mathrm{Y}} v_{\overline{\mathrm{K}} \mathrm{N}}\right\rangle} \\
& D_{1}:=\frac{\left\langle\sigma_{\mathrm{YN}}^{\overline{\mathrm{K}}+4 \pi} v_{\mathrm{Y} \overline{\mathrm{N}}}\right\rangle}{\left\langle\sigma_{\mathrm{N} \bar{N}}^{5 \pi} v_{\mathrm{N} N}\right\rangle} ; \quad D_{2}:=\frac{\left\langle\sigma_{\mathrm{\Xi} \overline{\mathrm{N}}}^{2 \overline{\mathrm{K}}+3 \pi} v_{\Xi \overline{\mathrm{N}}}\right\rangle}{\left\langle\sigma_{\mathrm{N} \overline{\mathrm{N}}}^{5 \pi} v_{\mathrm{N}}\right\rangle} ; \quad D_{3}:=\frac{\left\langle\sigma_{\Omega \overline{\mathrm{N}}}^{3 \overline{\mathrm{K}}+2 \pi} v_{\Omega \overline{\mathrm{N}}}\right\rangle}{\left\langle\sigma_{\mathrm{N} \overline{\mathrm{N}}}^{5 \pi} v_{\mathrm{N}}\right\rangle} \\
& R_{1}:=\frac{\rho_{\pi}^{\infty} \rho_{\Xi}^{\infty}}{\overline{\rho_{\Omega}^{\infty} \rho_{\mathrm{K}}}} ; \quad R_{2}:=\frac{\rho_{\pi}^{\infty} \rho_{\mathrm{Y}}^{\infty}}{\overrightarrow{\rho_{\Xi}^{\infty} \rho_{\mathrm{K}}^{\infty}}} ; \quad R_{3}:=\frac{\rho_{\pi}^{\infty} \rho_{\mathrm{N}}^{\infty}}{\overrightarrow{\rho_{\mathrm{Y}}^{\infty} \rho_{\mathrm{K}}}} ; \quad R_{4}:=\frac{\left(\rho_{\pi}^{\infty}\right)^{2}}{\rho_{\mathrm{K}}^{\infty} \rho_{\overline{\mathrm{K}}}^{\infty}} \\
& R_{5}:=\frac{\rho_{\overline{\mathrm{K}}}^{\infty} \rho_{\Xi}^{\infty}}{\overrightarrow{\rho_{\Omega}^{\infty} \rho_{\pi}}} ; \quad R_{6}:=\frac{\rho_{\overline{\mathrm{K}}}^{\infty} \rho_{\mathrm{Y}}^{\infty}}{\overrightarrow{\rho_{\pi}^{\infty} \rho_{\Xi}}} ; \quad R_{7}:=\frac{\rho_{\overline{\mathrm{K}}}^{\infty} \rho_{\mathrm{N}}^{\infty}}{\overrightarrow{\rho_{\mathrm{Y}} \rho_{\pi}}} \\
& R_{8}:=\frac{\rho_{\mathrm{N}}^{\infty} \rho_{\overline{\mathrm{N}}}^{\infty}}{\left(\rho_{\pi}^{\infty}\right)^{5}} ; \quad R_{9}:=\frac{\rho_{\mathrm{Y}}^{\infty} \rho_{\overline{\mathrm{N}}}^{\infty}}{\left(\rho_{\overline{\mathrm{K}}}^{\infty}\right)\left(\rho_{\pi}^{\infty}\right)} ; \quad R_{10}:=\frac{\rho_{\Xi}^{\infty} \rho_{\overline{\mathrm{N}}}^{\infty}}{\left(\rho_{\overline{\mathrm{K}}}^{\infty}\right)^{2}\left(\rho_{\pi}^{\infty}\right)^{3}} ; \quad R_{11}:=\frac{\rho_{\Omega}^{\infty} \rho_{\overline{\mathrm{N}}}^{\infty}}{\left(\rho_{\overline{\mathrm{K}}}^{\infty}\right)^{3}\left(\rho_{\pi}^{\infty}\right)^{2}},
\end{aligned}
$$

where the $\rho_{i}^{\infty}$ denote the equilibrium densities which are given by:

$$
\begin{aligned}
& \rho_{\Omega, \bar{\Omega}}^{\infty}=\frac{4 T^{3}}{2 \pi^{2}}\left(\frac{m_{\Omega}}{T}\right)^{2} K_{2}\left(\frac{m_{\Omega}}{T}\right) \lambda_{\mathrm{s}}^{ \pm 3}, \\
& \rho_{\Xi, \equiv}^{\infty}=\frac{4 T^{3}}{2 \pi^{2}}\left(\frac{m_{\Xi}}{T}\right)^{2} K_{2}\left(\frac{m_{\Xi}}{T}\right) \lambda_{\mathrm{q}}^{ \pm 1} \lambda_{\mathrm{s}}^{ \pm 2}, \\
& \rho_{\mathrm{Y}, \bar{Y}}^{\infty}=\frac{8 T^{3}}{2 \pi^{2}}\left(\frac{m_{\mathrm{Y}}}{T}\right)^{2} K_{2}\left(\frac{m_{\mathrm{Y}}}{T}\right) \lambda_{\mathrm{q}}^{ \pm 2} \lambda_{\mathrm{s}}^{ \pm 1},
\end{aligned}
$$




$$
\begin{aligned}
& \rho_{\mathrm{N}, \overline{\mathrm{N}}}^{\infty}=\frac{4 T^{3}}{2 \pi^{2}}\left(\frac{m_{\mathrm{N}}}{T}\right)^{2} K_{2}\left(\frac{m_{\mathrm{N}}}{T}\right) \lambda_{\mathrm{q}}^{ \pm 3}, \\
& \rho_{\mathrm{K}, \overline{\mathrm{K}}}^{\infty}=\frac{2 T^{3}}{2 \pi^{2}}\left(\frac{m_{\mathrm{K}}}{T}\right) K_{2}\left(\frac{m_{\mathrm{K}}}{T}\right) \lambda_{\mathrm{q}}^{ \pm 1} \lambda_{\mathrm{s}}^{\mp 1}, \\
& \rho_{\pi}^{\infty}=\frac{3 T^{3}}{2 \pi^{2}}\left(\frac{m_{\pi}}{T}\right)^{2}\left\{K_{2}\left(\frac{m_{\pi}}{T}\right)+\frac{1}{2} K_{2}\left(\frac{2 m_{\pi}}{T}\right)+\frac{1}{3} K_{2}\left(\frac{3 m_{\pi}}{T}\right)\right\} .
\end{aligned}
$$

Except for the case of pions, the densities are calculated in Boltzmann approximation. Note that $\mathrm{K}, \overline{\mathrm{K}}$ denotes the K-mesons containing an $\overline{\mathbf{s}}$, s quark, respectively.

One should notice that all reaction constants defined under (A12) are independent of the fugacities $\lambda_{\mathrm{q}}, \lambda_{\mathrm{s}}$ since these quantum numbers are conserved for each reaction. Therefore they only depend on the temperature and the masses of participating particles in the HG. The quantum number (chemical potential) dependence enters through the initial condition, in our case e.g. the assumption of equilibrated phase space of nucleons.

\section{Appendix B: Parameterization of strangeness production cross sections in hadronic gas}

Unfortunately a unique parameterization of measured cross sections [He83] over the whole range of the centre-of-mass energy is not feasible. The full energy region is divided into smaller intervals where simple parameterizations apply. In the following we adopt the parameterization of ref. [KR84] rather than that of refs. [ME82, CL84].

Specifically, we have chosen the following fits. In the case of the $\pi+p \rightarrow \vec{K}+Y$ reactions.

$$
\begin{aligned}
& \pi^{+} \mathrm{p} \rightarrow \Sigma^{+} \mathrm{K}^{+}: \\
& \sigma(\sqrt{s})=\left\{\begin{array}{lll}
A\left(\sqrt{s}-\sqrt{s_{0}}\right) ; & \sqrt{s_{0}}<\sqrt{s}<\sqrt{s_{\mathrm{m}}} ; & \sqrt{s_{0}}=1.683 \mathrm{GeV} \\
& \sqrt{s_{\mathrm{m}}}=1.934 \mathrm{GeV} ; \quad A=0.7 \mathrm{mb} / 0.218 \mathrm{GeV} \\
B \exp (-C \sqrt{s}) ; & \sqrt{s_{\mathrm{m}}}<\sqrt{s}<\sqrt{s_{\mathrm{K}}} ; & \sqrt{s_{\mathrm{K}}}=3 \mathrm{GeV} ; \\
& B=60.26 \mathrm{mb} ; \quad C=2.31 \mathrm{GeV}^{-1} \\
D \exp (-E \sqrt{s}) ; & \sqrt{s_{\mathrm{K}}}<\sqrt{s}<\infty ; \quad D=0.36 \mathrm{mb} ; \quad E=0.605 \mathrm{GeV}^{-1}
\end{array}\right. \\
& \pi^{-} \mathrm{P} \rightarrow \mathrm{K}^{0} \Lambda: \\
& \sigma(\sqrt{s})=\left\{\begin{array}{lll}
A\left(\sqrt{s}-\sqrt{s_{0}}\right) ; & \sqrt{s_{0}}<\sqrt{s}<\sqrt{s_{\mathrm{m}}} ; \quad \sqrt{s_{0}}=1.613 \mathrm{GeV} ; \\
B \exp (-C \sqrt{s}) ; & \sqrt{s_{\mathrm{m}}}=1.684 \mathrm{GeV} ; \quad A=0.9 \mathrm{mb} / 0.091 \mathrm{GeV} \\
& B=436.3 \mathrm{mb} ; \quad C=4.154 \mathrm{GeV}^{-1} \\
D \exp (-E \sqrt{s}) ; & \sqrt{s_{\mathrm{K}}}<\sqrt{s}<\infty ; \quad D=0.314 \mathrm{mb} ; \quad E=0.301 \mathrm{GeV}^{-1}
\end{array}\right.
\end{aligned}
$$




$$
\pi^{-} \mathrm{P} \rightarrow \Sigma^{0} \mathbf{K}^{0}:
$$

$$
\sigma(\sqrt{s})=\left\{\begin{array}{lll}
A\left(\sqrt{s}-\sqrt{s_{0}}\right) ; & \sqrt{s_{0}}<\sqrt{s}<\sqrt{s_{\mathrm{m}}} ; & \sqrt{s_{0}}=1.689 \mathrm{GeV} ; \\
B \exp (-C \sqrt{s}) ; & \sqrt{s_{\mathrm{m}}}=1.722 \mathrm{GeV} ; \quad A=10.6 \mathrm{mb} / \mathrm{GeV} \\
& B=13.7 \mathrm{mb} ; \quad C=1.92 \mathrm{GeV}^{-1} \\
\pi^{-} \mathrm{P} \rightarrow \Sigma^{-} \mathrm{K}^{+}: & \sqrt{s_{\mathrm{K}}}<\sqrt{s}<\infty ; \quad D=0.188 \mathrm{mb} ; \quad E=0.611 \mathrm{GeV}^{-1}
\end{array}\right.
$$

$$
\sigma(\sqrt{s})=\left\{\begin{array}{lll}
F(\text { constant }) ; & \sqrt{s_{0}}<\sqrt{s}<\sqrt{s_{\mathrm{m}}} ; & \sqrt{s_{0}}=1.691 \mathrm{GeV} \\
D \exp (-E \sqrt{s}) ; & \sqrt{s_{\mathrm{K}}}<\sqrt{s}<\infty ; & \sqrt{s_{\mathrm{m}}}=\sqrt{s_{\mathrm{K}}}=1.9 \mathrm{GeV} \\
& D=309.06 \mathrm{mb} ; \quad E=3.77 \mathrm{GeV}^{-1} .
\end{array}\right.
$$

In figs. B1a-B1d we show these respective fits with data taken from CERN-HERA Reports [HE83].
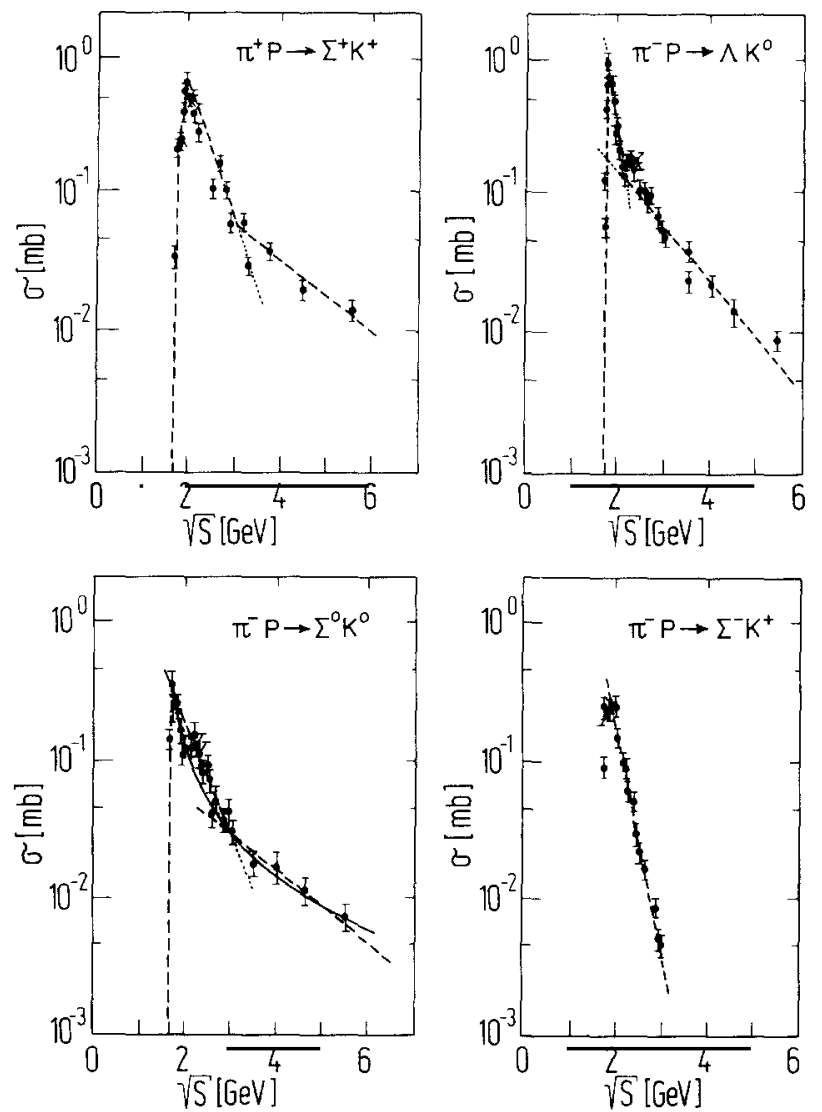

Fig. B1. Cross sections for the strangeness production process. Data are taken from CERN-HERA Reports [HE83]. Our parameterization as outlined in appendix B. 
Since we are now interested in an isospin averaged cross section, we have adopted the approach of ref. [CL84] and taken the mean $\pi \mathrm{N} \rightarrow \overline{\mathrm{K}} \mathrm{Y}$ cross section as:

$$
\bar{\sigma}(\pi \mathrm{N} \rightarrow \overline{\mathrm{K}} \mathrm{Y})=\frac{1}{4}\left\{\sigma\left(\pi^{+} \mathrm{p} \rightarrow \Sigma^{+} \mathrm{K}^{+}\right)+\sigma\left(\pi^{-} \mathrm{p} \rightarrow \Sigma^{0} \mathrm{~K}^{0}\right)+\sigma\left(\pi^{-} \mathrm{p} \rightarrow \Sigma^{-} \mathrm{K}^{-}\right)\right\} .
$$

In the case of strangeness exchange cross sections

$$
\overline{\mathrm{K}} \mathrm{N} \rightarrow \mathrm{Y} \pi
$$

we only have data for the mostly measured reaction

$$
\mathrm{K}^{-} \mathrm{p} \rightarrow \Lambda \pi^{0}
$$

and few data points for

$$
\mathrm{K}^{-} \mathrm{N} \rightarrow \Lambda \pi^{-}
$$

as displayed in fig. B2.

In order to obtain an isospin averaged cross section, we assume

$$
\bar{\sigma}(\overline{\mathrm{K}} \mathrm{N} \rightarrow \mathrm{Y} \pi)=\frac{1}{2}\left\{\sigma\left(\mathrm{K}^{-} \mathrm{p} \rightarrow \Lambda \pi^{0}\right)+\sigma\left(\mathrm{K}^{-} \mathrm{N} \rightarrow \Lambda \pi^{-}\right)\right\} .
$$

The fits for the shown cross sections (fig. B2) are:

$$
\mathrm{K}^{-} \mathrm{p} \rightarrow \pi^{0} \Lambda
$$

$$
\sigma\left(\mathrm{K}^{-} \mathrm{p} \rightarrow \Lambda \pi^{0}\right)=\left\{\begin{array}{lll}
B \exp (-C \sqrt{s}) ; & \sqrt{s_{\mathrm{m}}}<\sqrt{s}<\sqrt{s_{\mathrm{K}}} ; & \sqrt{s_{\mathrm{m}}}=1.473 \mathrm{GeV} \\
& B=1479.53 \mathrm{mb} & C=3.377 \mathrm{GeV}^{-1} \\
D \exp (-E \sqrt{s}) ; & \sqrt{s_{\mathrm{K}}}<\sqrt{s}<\infty ; & D=0.3 \mathrm{mb} ; \quad E=0.72 \mathrm{GeV}^{-1}
\end{array}\right.
$$
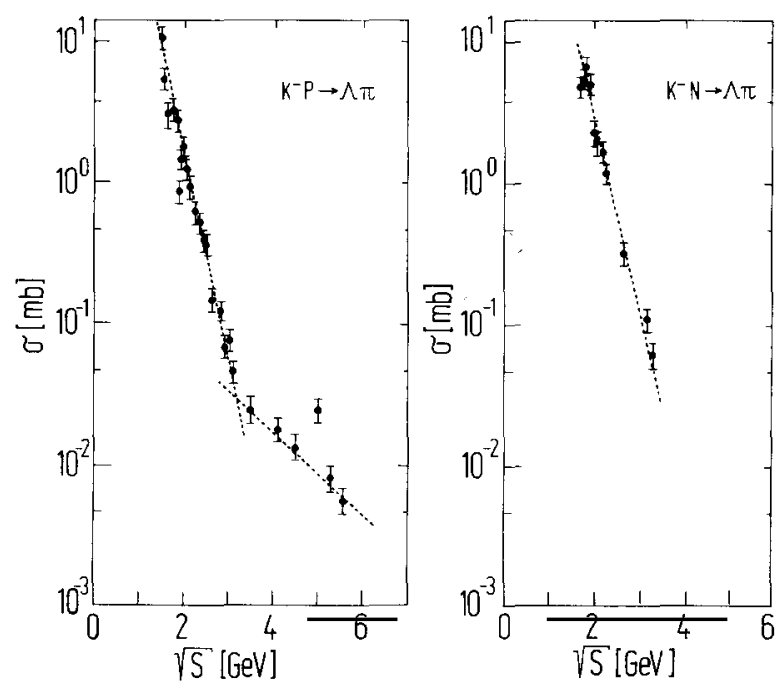

Fig. B2. Data and our fits to the strangeness exchange cross sections. Data are taken from [HE83]. 


$$
\begin{aligned}
& \mathrm{K}^{-} \mathrm{n} \rightarrow \Lambda \pi^{-}: \\
& \sigma\left(\mathrm{K}^{-} \mathrm{n} \rightarrow \Lambda \pi^{-}\right)=D \exp (-E \sqrt{s}) ; \quad \sqrt{s_{\mathrm{K}}}<\sqrt{s}<\infty ; \quad \sqrt{s_{\mathrm{K}}}=\sqrt{s_{\mathrm{m}}}=1.699 \mathrm{GeV} ; \\
& \\
& E=3.063 \mathrm{GeV}^{-1} ; \quad D=1132.27 \mathrm{mb} .
\end{aligned}
$$

These fits are also shown in figs. B2a and B2b.

In the case of annihilation cross sections into five pions we only have information about the channel

$$
\mathrm{p} \overline{\mathrm{p}} \rightarrow 5 \pi\left(\pi^{+}+2 \pi^{-}+\pi^{0}\right)
$$

and we took

$$
\bar{\sigma}(\mathrm{N} \overline{\mathrm{N}} \rightarrow 5 \pi) \simeq \sigma\left(\mathrm{p} \overline{\mathrm{p}} \rightarrow 2 \pi^{+}+2 \pi^{-}+\pi^{0}\right)
$$

and fit the data to the form

$$
\begin{aligned}
& \sigma\left(\mathrm{p} \overrightarrow{\mathrm{p}} \rightarrow 2 \pi^{+}+2 \pi^{-}+\pi^{0}\right)=D \exp (-E \sqrt{s}) ; \quad \sqrt{s_{\mathrm{K}}}<\sqrt{s}<\infty \\
& \sqrt{s_{\mathrm{K}}}=\sqrt{s_{\mathrm{m}}}=1.985 \mathrm{GeV} ; \quad D=871.72 \mathrm{mb} ; \quad \mathrm{E}=1.95 \mathrm{GeV}^{-1}
\end{aligned}
$$

The fit is shown in fig. B3.

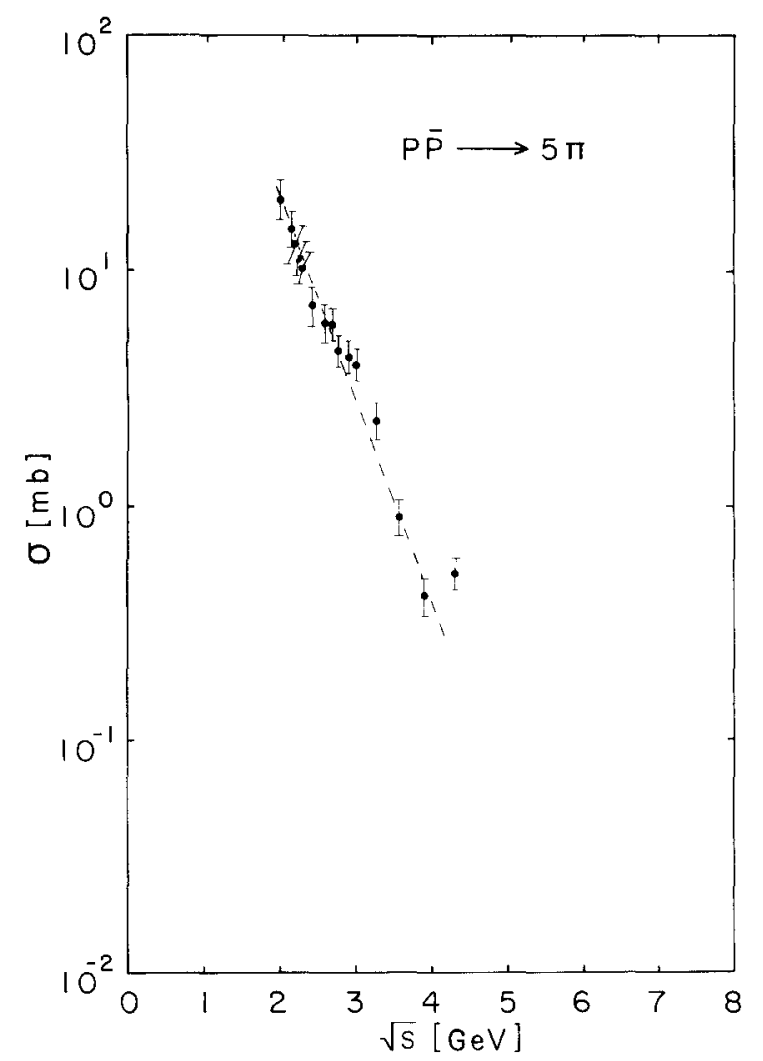

Fig. B3. Data and our fit to the baryon annihilation channel with data from [HE83] 


\section{References}

Surveys of the status of Quark-Gluon Plasma research are found in the proceedings of the "Quark Matter" conferences: Bielefeld: 1980 in [Sa81] and 1982 in [JS82]; Brookhaven (1983) in Nucl. Phys. A418 (1984); and Helsinki (1984) in Springer Tracts Vol. 221. See in particular R. Hagedorn (p. 53ff) in this volume with regard to the early development of this subject matter.

[ABS84] F. Asai, H. Bandō and M. Sano, Phys. Lett. 145B (1984) 19.

[AGIS83] B. Anderson, G. Gustafson, G. Ingelman and T. Sjöstrand, Phys. Reports 97 (1983) 31.

[Ak85] T. Akesson et al. (Axial Field Spectrometer collaboration), Phys. Rev. Lett. 55 (1985) 2535.

[AKM80] R. Anishetty, P. Koehler and L. McLerran, Phys. Rev. D22 (1980) 2793.

[An84] M. Anikina et al., Z. Physik C25 (1984) 1.

[AR84] A.T.M. Aerts and J. Rafelski, Phys. Lett. 148B (1984) 337.

[AR85] A.T. Aerts and J. Rafelski, Strange Hadrons in the MIT Bag Model, preprint CERN-TH 4160/85 and UCT-TP 27-2/85.

[As81] F. Asai, Nucl. Phys. A365 (1981) 519.

[AS81] F. Asai and M. Sano, Prog. Theor. Phys. 66 (1981) 251.

[ASS81] F. Asai, H. Sato and M. Sano, Phys. Lett. 98B (1981) 19.

[Ba74] W.F. Baker et al., Nucl. Phys. B51 (1974) 303.

[Ba83] D.S. Barton et al., Phys. Rev. D27 (1983) 2580.

[Ba83a] G. Baym, B.L. Friman, J.P. Blaizot, M. Soyeur and W. Czyz, Nucl. Phys. A407 (1983) 541.

[BG84] W. Busza and A.S. Goldhaber, Phys. Lett. 139B (1984) 235.

[BGM83] B. Banerjee, N.K. Glendenning and T. Matsui, Phys. Lett. 127B (1983) 453.

[Bi82] T.S. Biro, B. Lukacs, J. Zimanyi and H.W. Barz, Nucl. Phys. A386 (1982) 617; Z. Physik A311 (1983) 311.

[Bj83] J.D. Bjorken, Phys. Rev. D27 (1983) 140.

[BI84] H.W. Barz and H. Iwe, Phys. Lett. 143B (1984) 55.

[Bo79] M. Bourquin et al., Nucl. Phys. B153 (1979) 13.

[Bo80] M. Bourquin et al., Z. Physik C5 (1980) 275.

[BS81] R. Bock and R. Stock (eds.), Workshop on Future Relativistic Heavy Ion Experiments, GSI-Report 81-6.

[BZ82] T. Biro and J. Zimanyi, Phys. Lett. 113B (1982) 6 and private communication.

[BZ83] T. Biro and J. Zimanyi, Nucl. Phys. A395 (1983) 525.

[Ca74] P. Capiluppi et al., Nucl. Phys. B79 (1974) 189.

[Ca73] P. Carruthers, Coll. Pheno. 1 (1973) 147.

[Ca83] P. Carruthers, Phys. Rev. Lett. 50 (1983) 1179.

[CCR84] J.W. Clark, J. Cleymans and J. Rafelski, Clustered Quark Matter, preprint UCT-TP-18-2, Cape Town 1984.

[CDH83] J. Cleymans, M. Dechantsreiter and F. Halzen, Z. Physik C17 (1983) 341.

[CGS86] J. Cleymans, R.V. Gavai and E. Suhonen, Phys. Reports 130 (1986) 217.

[Ch74] A. Chodos, R.L. Jaffe, K. Johnson, C.B. Thorn and V.F. Weisskopf, Phys. Rev. D9 (1974) 3471.

[Ch78] S.A. Chin, Phys. Lett. 78B (1978) 552.

[Ch82] S.A. Chin, Phys. Lett. 119B (1982) 51.

[CK84] L.P. Csernai and J. Kapusta, Phys. Rev. D29 (1984) 2664.

[CKR77] B.L. Combridge, J. Kripfganz and J. Ranft, Phys. Lett. 70B (1977) 234.

[CL84] J. Cugnon and R.M. Lombard, Phys. Lett. 134B (1984) 392; Nucl. Phys. A422 (1984) 635.

[CNN79] A. Casher, H. Neuberger and S. Nussinov, Phys. Rev. D20 (1979) 179.

[Co79] B.L. Combridge, Nucl. Phys. B151 (1979) 429.

[CP84] J. Cleymans and R. Philippe, Z. Physik C22 (1984) 271.

[CV84] J. Cleymans and C. Vanderzande, Phys. Lett. 147B (1984) 186.

[deG75] T. deGrand, R.L. Jaffe, K. Johnson and J. Kiskis, Phys. Rev. D12 (1975) 2060.

[DG81] G. Domokos and J.I. Goldman, Phys. Rev. D23 (1981) 203.

[DGER85] C. Derreth, W. Greiner, H.-Th. Elze and J. Rafelski, Phys. Rev. C31 (1985) 1360.

[DGS85] S. Date, M. Gyulassy and H. Sumiyoshi, Phys. Rev. D32 (1985) 619

[DR82] M. Danos and J. Rafelski, Formation of Quark-Gluon Plasma at Central Rapidity, University of Frankfurt preprint UFTP94/1982 (unpublished); extended manuscript: Baryon-rich Quark-Gluon Plasma in Nuclear Collisions, UCT-TP7/84 (unpublished).

[DR83] M. Danos and J. Rafelski, Phys. Rev. D27 (1983) 671.

[DR85] D.W. Duke and R.G. Roberts, Phys. Reports 120 (1985) 275.

[EGR80] T. Elze, W. Greiner and J. Rafelski, J. Phys. G6 (1980) L419.

[EGR83] T. Elze, W. Greiner and J. Rafelski, Phys. Lett. 124B (1983) 515. 
[EGR84] T. Elze, W. Greiner and J. Rafelski, Z. Physik C24 (1984) 361.

[FF78] R.D. Fields and R.P. Feynman, Nucl. Phys. B136 (1978).

[Ge78] H.M. Georgi, S.L. Glashow, M.E. Machacek and D.V. Nanopoulos, Ann. Phys. (N.Y.) 114 (1978) 273

[GKR84] W. Greiner, P. Koch and J. Rafelski, Phys. Lett. 145B (1984) 142.

[GL82] J. Gasser and H. Leutwyler, Phys. Reports 87 (1982) 77.

[GM85] N.K. Glendenning and T. Matsui, Phy. Rev. D28 (1983) 2890.

[GP76] H. Georgi and H.D. Politzer, Phys. Rev. D14 1829 (1976).

[GR85] N.K. Glendenning and J. Rafelski, Phys. Rev. C31 (1985) 823.

[Ha65] R. Hagedorn, Suppl. Nuovo Cim. 3 (1965) 147.

[Ha68] R. Hagedorn, Il Nuovo Cimento 56A (1968) 1027.

[Ha81] J.W. Harris, A. Sandoval, R. Stock, H. Stroebele, R.E. Renfordt, J.V. Geaga, H.G. Pugh, L.S. Schroeder, K.L. Wolf and A. Dacal, Phys. Rev. Lett. 47 (1981) 229.

[HE83] CERN-HERA Reports:

Compilation of cross sections for

(a) $\pi^{-}$and $\pi^{+}$induced reactions, CERN-HERA 83-01 (1983);

(b) $\mathrm{K}^{-}$and $\mathrm{K}^{+}$induced reactions, CERN-HERA 83-0 (1983);

(c) $\mathrm{p}$ and $\overline{\mathrm{p}}$ induced reactions, CERN-HERA 84-01 (1984).

[He85] U. Heinz, P.R. Subramanian and W. Greiner, paper contributed to the Workshop on Experiments for RHIC, Brookhaven, April 1985.

[HK85] R.C. Hwa and K. Kajantie, Phys. Rev. D32 (1985) 1109.

[HR68] R. Hagedorn and J. Raft, Suppl. Nuovo Cim. 6 (1968) 169.

[HR80] R. Hagedorn and J. Rafelski, Phys. Lett. 97B (1980) 136.

[HR84] R. Hagedorn and K. Redlich, Z. Physik C27 (1985) 541.

[HSG84] U. Heinz, P.R. Subramanian and W. Greiner, Z. Physik A318 (1984) 247.

[Jo75] K. Johnson, Acta Phys. Pol. B61 (1975) 865.

[JS82] M. Jacob and H. Satz (eds.), Quark Matter Formation and Heavy Ion Collisions (World Scientific Publ., Singapore 1982).

[Ka85] B. Kämpfer, H.W. Barz, L. Münchow and B. Lukacs, preprint KFKI-1985-65, Budapest 1985.

[KB73] K. Kajantile and E. Byckling, Particle Kinematics (Wiley and Sons 1973).

[KM85] J. Kapusta and A. Mekjian, Ultrarelativistic Nucleus-Nucleus Collisions are Strange, UMSI85/6, to be published.

[KMR86] P. Koch, B. Müller and J. Rafelski, Strangeness Production and Evolution in Quark Gluon Plasma, Z. Physik A, in press.

[Ko81] C.M. Ko, Phys. Rev. C23 (1981) 2760.

[Ko83] C.M. Ko, Phys. Lett. 120B (1983) 294.

[Ko84] C.M. Ko, Phys. Lett. 138B (1984) 361.

[Ko86] P. Koch, B. Müller and J. Rafelski, to be published (1986).

[KR84] P. Koch and J. Rafelski, Time Evolution of Strange Particle Densities in Hot Hadronic Matter, UCT-TP22.

[KR85] P. Koch and J. Rafelski, Nucl. Phys. A444 (1985) 678.

[KRa85] P. Koch and J. Rafelski, Why the Hadronic Gas Description of Hadronic Reactions Works: The Example of Strange Hadrons, UCT-TP 26-2/1985, S. Afr. (1986) in press.

[KRG83] P. Koch, J. Rafelski and W. Greiner, Phys. Lett. 123B (1983) 151.

[KRR83] K. Kajantie, R. Raitio and P.V. Ruuskanen, Nucl. Phys. B222 (1983) 152.

[KS85] H. Kowalski, H. Sprecht et al. (NA34 (CERN) collaboration), in: Proc. 2nd Int. Nucl. Collisions Conf., Visby 85, Nucl. Phys. A (1985).

[Le81] T.D. Lee, Particle Physics and Introduction to Field Theory (Horwood Pub., Chur 1981).

[Ma85] T. Matsui, B. Svetitsky and L. McLerran, Strangeness production in ultrarelativistic heavy ion collisions: I. Chemical kinetics in the quark-gluon plasma, MIT preprint CTP-1320, November 1985.

[Me82] A.Z. Mekjian, Nucl. Phys. A384 (1982) 492.

[ME85] B. Müller and J.M. Eisenberg, Nucl. Phys. A435 (1985) 791.

[MM84] T. Munehisa and Y. Munehisa, Strange Particle Production at an Intermediate Temperature, Preprint YAMANASHI 84-01, 1984.

[Mü85] B. Müller, Springer Lecture Notes in Physics 225 (1985).

[MuK83] K.H. Müller, Nucl. Phys. A395 (1983) 509.

[NW81] D. Nachtmann and W. Wetzel, Nucl. Phys. B187 (1981) 333.

[Pi82] R. Pisarski, Phys. Lett. 110B (1982) 155.

[PMG85] G. Plunien, B. Müller and W. Greiner, Phys. Reports 134 (1986) 87.

[Po76] H.D. Politzer, Nucl. Phys. B117 (1976) 397.

[Pr73] S.D. Protopopescu et al., Phys. Rev. D7 (1973) 1279.

[Ra81] J. Rafelski, in [BS81] p. 282.

[Ra82] J. Rafelski, Phys. Reports 88 (1982) 331.

[Ra83] J. Rafelski, S. Afr. J. Phys. 6 (1983) 37. 
[Ra84] J. Rafelski, Nucl. Phys. A418 (1984) 215.

[RD80] J. Rafelski and M. Danos, Phys. Lett. 97B (1980) 279.

[RD83] J. Rafelski and M. Danos, Perspectives in High-Energy Nuclear Collisions, Report NBSIR 83-2725, Washington 1983.

[RD85] J. Rafelski and M. Danos, in: Springer Lecture Notes in Physics 231 (1985) pp. 361-455.

[Re84] H.J. Reusch, Z. Physik C26 (1984) 105.

[Re85] K. Redlich, Z. Physik C27 (1985) 633.

[RH80] J. Rafelski and R. Hagedorn, From Hadron Gas to Quark Matter II, CERN-TH 2969 (1980), published in [Sa81].

[RK80] J. Randrup and C.M. Ko, Nucl. Phys. A343 (1980) 519.

[RM82] J. Rafelski and B. Müller, Phys. Rev. Lett. 48 (1982) 1066.

[RM86] J. Rafelski and B. Müller, Strangeness Production and Evolution in Quark Gluon Plasma, UCT-TP 38/1986.

[Ro75] A.M. Rossi, G. Vannini, A. Bussiere, E. Albini, D. D'Alessandro and G. Giacomelli, Nucl. Phys. B84 (1975) 269.

[RRY85] L.J. Reinders, H. Rubinstein and S. Yasaki, Phys. Reports 127 (1985) 1.

[Sa81] H. Satz (ed.), Thermodynamics of Quarks and Hadrons (North-Holland, Amsterdam 1981).

[SC85] P.J. Siemens and S.A. Chin, Phys. Rev. Lett. 55 (1985) 1266.

[Sc78] P. Scubic et al., Phys. Rev. D18 (1978) 3115.

[Sch82] S. Schnetzer et al., Phys. Rev. Lett. 49 (1982) 989.

[Sch84] A. Schnabel, Diploma thesis, Frankfurt 1984.

[Sh81] M.A. Shifman, Z. Physik C9 (1981) 347.

[Sh81a] Z.V. Shuryak, Phys. Lett. 107B (1981) 103.

[Sh82] A. Shor et al., Phys. Rev. Lett. 48 (1982) 1597.

[Sh85] A. Shor, Phys. Rev. Lett. 54 (1985) 1122.

[Si78] J. Singh et al., Nucl. Phys. B140 (1978) 189.

[To68] B. Touschek, Nuovo Cimento B58 (1968) 295.

[vHP75] L. Van Hove and S. Pokorski, Nucl. Phys. B86 (1975) 243.

[Zv84] W. Zwermann, B. Schürmann, K. Dietrich and E. Martschew, Phys. Lett. 134B (1984) 397.

[ZS84] W. Zwermann and B. Schürmann, Nucl. Phys. A423 (1984) 525.

\section{Notes added in proof}

Several relevant articles have come to our attention following the completion of this report. These works include:

1) Matsui et al. [MA86], extending their earlier work [MA85], have studied strangeness evolution in the framework of Bjorken's hydrodynamical description of the spacetime evolution in the central rapidity region of ultrarelativistic heavy ion collisions. Their framework is similar to that of Kapusta and Mekjian [KM85], in particular, they only consider pions and kaons in the hadronic phase, but not baryons. Their results are very similar to [KM85]; when a plasma state is formed the resultant $K / \pi$ ratio will be enhanced roughly by a factor of 4 from that in pp collisions as has been predicted by [GR85]. They also do not consider hadronic chemical reactions in the final phase and neglect gluon fragmentation.

2) The influence of strange particles on critical thermodynamical variables arising from a model for the phase transition from hadron gas to quark gluon plasma is studied by [LRH86] and was found to be small, in line with naive expectations.

3) In a recent preprint Heinz, Subramanian, Stöcker and Greiner [He86] have also pointed out the importance of entropy conservation for the hadronization scenario. They conclude that their earlier results [He85] concerning antinuclei production must be taken with caution, but no new results are presented.

4) Biro and Zimanyi [BZ86] have investigated strange hadron production in the context of a mean-field model of the hadronic phase.

5) The experimental situation concerning strange hadrons $(K, \Lambda)$ in relativistic nuclear collisions up to $2.1 \mathrm{GeV} / \mathrm{u}$. is reviewed by Stock in his comprehensive review article [St86]. He also discusses the possible use of $\Lambda$-polarization as a probe for collision dynamics. 
[BZ86] T.S. Biro and J. Zimanyi, Phys. Lett. 167B (1986) 271.

[He86] U. Heinz, P.R. Subramanian, H. Stöcker and W. Greiner, Formation of antimatter clusters in the hadronization phase transition, BNL preprint.

[LRH86] K.S. Lee, M.J. Rhoades-Brown and U. Heinz, Phys. Lett. 174B (1986) 123.

[MA86] T. Matsui, B. Svetitsky and L.D. McLerran, Strangeness production in ultra relativistic heavy ion collisions: II. Evolution of flavour composition in scaling hydrodynamics, MIT preprint CTP-1344, April 1986.

[St86] R. Stock, Phys. Reports 135 (1986) 261. 